\title{
Chiral Spiro Phosphoric Acid-Catalyzed Friedel-Crafts Conjugate Addition/Enantioselective Protonation Reactions
}

\author{
Yi-Pan Li, Zi-Qi Li, Biying Zhou, Mao-Lin Li, Xiao-Song Xue, Shou-Fei Zhu, \\ Qi-Lin Zhou* \\ State Key Laboratory and Institute of Elemento-Organic Chemistry, College of \\ Chemistry, Nankai University, Tianjin 300071, China \\ Email:sfzhu@nankai.edu.cn;qlzhou@nankai.edu.cn
}

\section{DFT Calculation Study}

\section{Computational Methods}

\section{$\underline{\text { DFT Methods }}$}

All DFT theoretical calculations have been carried out using the Gaussian 09 program package. The $\mathrm{B} 3 \mathrm{LYP}^{1}$ method with $6-31 \mathrm{G}^{*}$ basis set has been selected for geometry optimizations and calculation of Gibbs energy corrections at $258 \mathrm{~K}\left(-15^{\circ} \mathrm{C}\right)$. Final energies were retrieved from single-point calculations at the B3LYP/6-311+G** level, including the GD3BJ dispersion correction scheme developed by Grimme. ${ }^{2}$ All structures have been optimized considering solvent effects using the $\mathrm{SMD}^{3}$ model for Chlorobenzene. Reaction paths were traced by the intrinsic reaction coordinate method for all transition states. All energetics reported throughout the text are in kcal $/ \mathrm{mol}$. Structures were generated using CYLview. ${ }^{4}$

\section{Complete Citation for Gaussian 09}

Gaussian 09, Revision D.01,

Frisch, M. J.; Trucks, G. W.; Schlegel, H. B.; Scuseria, G. E.; Robb, M. A.; Cheeseman, J. R.; Scalmani, G.; Barone, V.; Mennucci, B.; Petersson, G. A.; Nakatsuji, H.; Caricato, 
M.; Li, X.; Hratchian, H. P.; Izmaylov, A. F.; Bloino, J.; Zheng, G.; Sonnenberg, J. L.; Hada, M.; Ehara, M.; Toyota, K.; Fukuda, R.; Hasegawa, J.; Ishida, M.; Nakajima, T.; Honda, Y.; Kitao, O.; Nakai, H.; Vreven, T.; Montgomery, J. A., Jr.; Peralta, J. E.; Ogliaro, F.; Bearpark, M.; Heyd, J. J.; Brothers, E.; Kudin, K. N.; Staroverov, V. N.; Keith, T.; Kobayashi, R.; Normand, J.; Raghavachari, K.; Rendell, A.; Burant, J. C.; Iyengar, S. S.; Tomasi, J.; Cossi, M.; Rega, N.; Millam, J. M.; Klene, M.; Knox, J. E.; Cross, J. B.; Bakken, V.; Adamo, C.; Jaramillo, J.; Gomperts, R.; Stratmann, R. E.; Yazyev, O.; Austin, A. J.; Cammi, R.; Pomelli, C.; Ochterski, J. W.; Martin, R. L.; Morokuma, K.; Zakrzewski, V. G.; Voth, G. A.; Salvador, P.; Dannenberg, J. J.; Dapprich, S.; Daniels, A. D.; Farkas, O.; Foresman, J. B.; Ortiz, J. V.; Cioslowski, J.; Fox, D. J. Gaussian 09, Revision D.01; Gaussian, Inc.: Wallingford, CT, 2013. 
Table S4. Sum of computed energies of stationary points (For Figure S1)

\begin{tabular}{cccccc}
\hline entry & Structure & $\Delta \mathrm{G}_{\text {sol }}$ (a.u.) & $\begin{array}{c}\Delta \Delta \mathrm{G}_{\text {sol }} \\
(\mathrm{kcal} / \mathrm{mol})\end{array}$ & $\Delta \mathrm{H}_{\text {sol }}($ a.u. $)$ & $\begin{array}{c}\Delta \Delta \mathrm{H}_{\text {sol }} \\
(\mathrm{kcal} / \mathrm{mol})\end{array}$ \\
\hline 1 & $\mathbf{1 a}$ & -536.376939954 & $\mathrm{~N} / \mathrm{A}$ & -536.340474954 & N/A \\
2 & $\mathbf{2 a}$ & -363.855462874 & $\mathrm{~N} / \mathrm{A}$ & -363.824478874 & N/A \\
3 & $(S)-3 g$ & -2390.331811900 & $\mathrm{~N} / \mathrm{A}$ & -2390.228066900 & N/A \\
4 & INT-I' & -3290.575689500 & -7.2 & -3290.439245500 & -29.0 \\
5 & INT-I & -3290.573211080 & -5.6 & -3290.433269080 & -25.3 \\
6 & TS-I' & -3290.552846740 & 7.1 & -3290.422359740 & -18.4 \\
7 & TS-I & -3290.551121890 & 8.2 & -3290.418530890 & -16.0 \\
8 & INT-II' & -3290.570180610 & -3.7 & -3290.438876610 & -28.8 \\
9 & INT-II & -3290.568087260 & -2.4 & -3290.435858260 & -26.9 \\
10 & TS-II' & -3290.548302480 & 10.0 & -3290.418502480 & -16.0 \\
11 & TS-II & -3290.551208190 & 8.2 & -3290.420884190 & -17.5 \\
12 & INT-III & -3290.569809080 & -3.5 & -3290.436146080 & -27.1 \\
13 & TS-IIII-R & -3290.553211180 & 6.9 & -3290.421465180 & -17.8 \\
14 & TS-III-S & -3290.556667360 & 4.7 & -3290.425958360 & -20.7 \\
15 & INT-IV-R & -3290.584289340 & -12.6 & -3290.450152340 & -35.9 \\
16 & INT-IV-S & -3290.590648840 & -16.6 & -3290.455460840 & -39.2 \\
17 & (S)-4aa & -900.248240444 & -9.9 & -900.198416444 & -21.0 \\
\hline
\end{tabular}




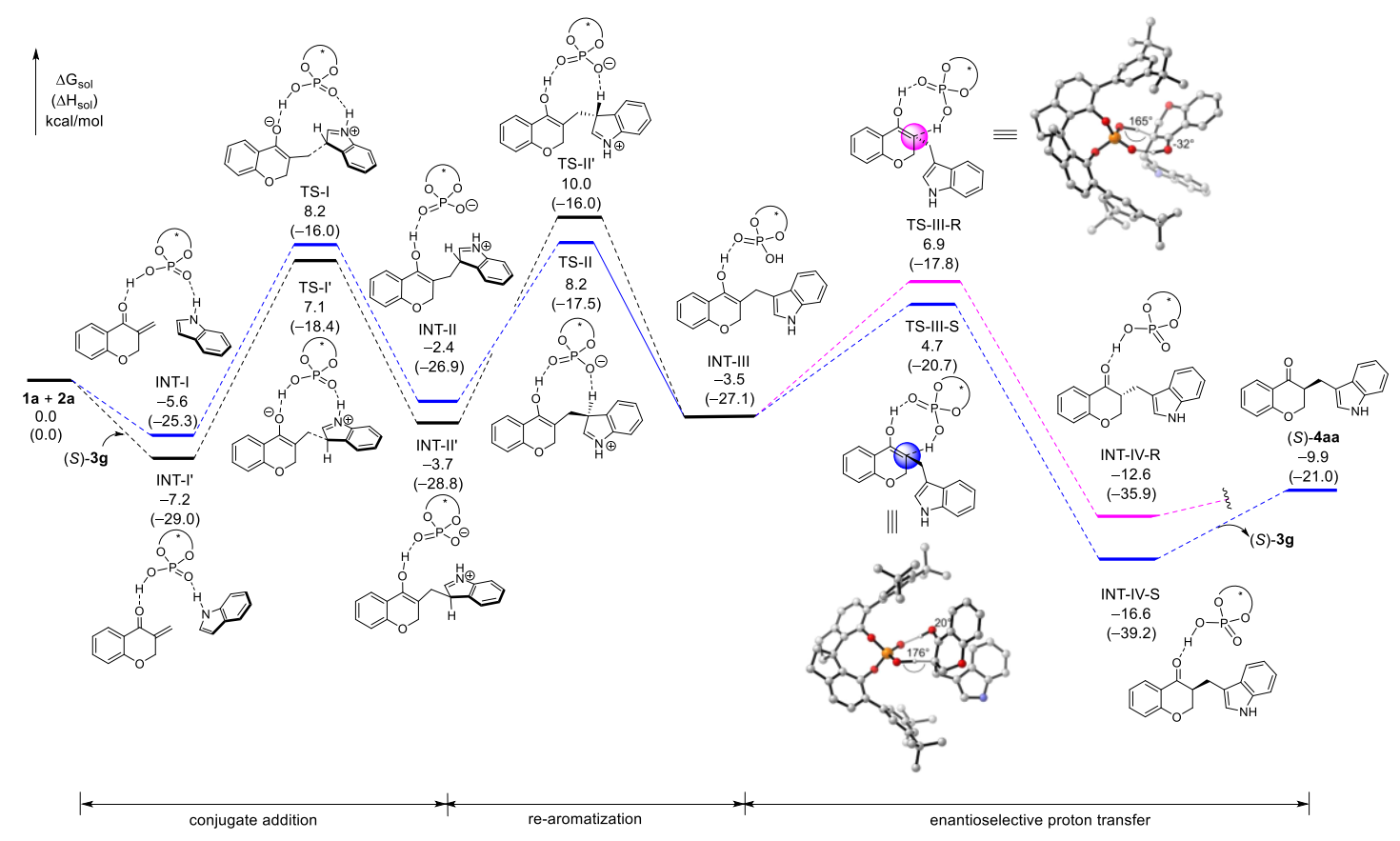

Figure S1. The computed energy surfaces for $(S)$-3g catalyzed Friedel-Crafts conjugated addition of 2a to 1a. Optimized structures of key stationary points with key bond length (in $\AA$ ). Trivial H atoms have been omitted for clarity.

Note: Two possible pathways for generation of the prochiral enol intermediate INT-III are calculated. The energy barrier between TS-II and TS-II' is $1.8 \mathrm{kcal} / \mathrm{mol}$. Therefore, the energy lower pathway is more favored (blue line). 
Table S5. Sum of computed energies of stationary points (For Figure S2)

\begin{tabular}{cccccc}
\hline entry & Structure & $\Delta \mathrm{G}_{\text {sol }}$ (a.u.) & $\begin{array}{c}\Delta \Delta \mathrm{G}_{\text {sol }} \\
(\mathrm{kcal} / \mathrm{mol})\end{array}$ & $\Delta \mathrm{H}_{\text {sol }}$ (a.u. $)$ & $\begin{array}{c}\Delta \Delta \mathrm{H}_{\text {sol }} \\
(\mathrm{kcal} / \mathrm{mol})\end{array}$ \\
\hline 1 & INT-I'-nc & -3290.574336880 & -6.4 & -3290.438527880 & -28.6 \\
2 & INT-I-nc & -3290.575662020 & -7.2 & -3290.437082020 & -27.6 \\
3 & TS-I'-nc & -3290.546856890 & 10.9 & -3290.415756890 & -14.3 \\
4 & TS-I-nc & -3290.546342830 & 11.2 & -3290.413920830 & -13.1 \\
\hline
\end{tabular}

nc refers to non-coordinating.

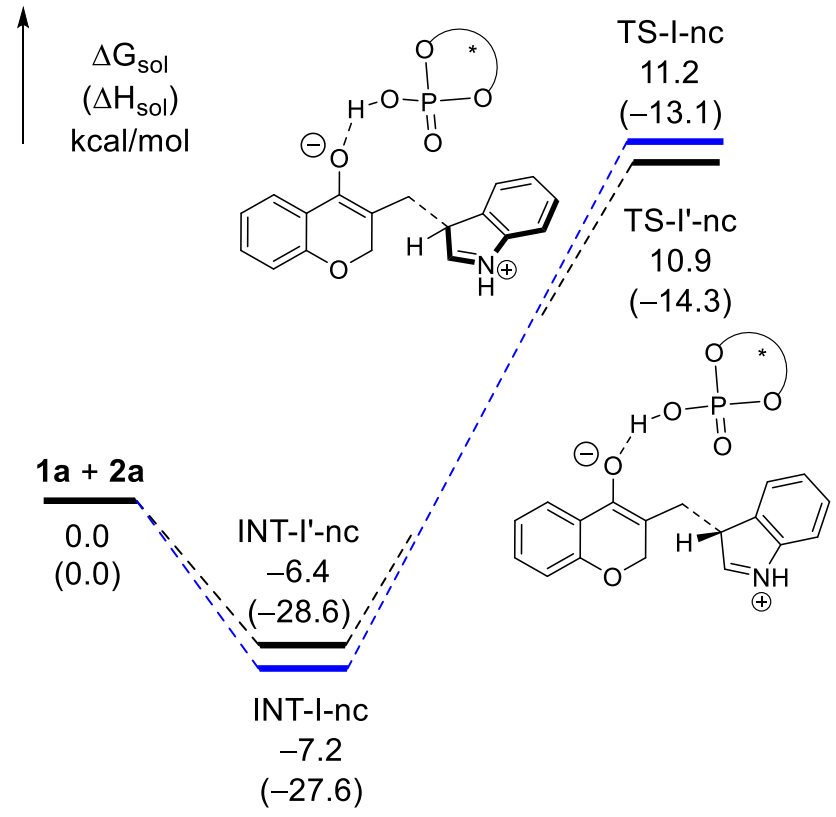

Figure S2. The computed energy surfaces for transition state without coordination between indole $N-\mathrm{H}$ and $(S)-3 g$. 
Table S6. Sum of computed energies of stationary points (For Figure S3)

\begin{tabular}{cccccc}
\hline entry & Structure & $\Delta \mathrm{G}_{\text {sol }}$ (a.u.) & $\begin{array}{c}\Delta \Delta \mathrm{G}_{\text {sol }} \\
(\mathrm{kcal} / \mathrm{mol})\end{array}$ & $\Delta \mathrm{H}_{\text {sol }}($ a.u. $)$ & $\begin{array}{c}\Delta \Delta \mathrm{H}_{\text {sol }} \\
(\mathrm{kcal} / \mathrm{mol})\end{array}$ \\
\hline 1 & INT-III'-dt & -3290.564992730 & -0.5 & -3290.432533730 & -24.8 \\
2 & INT-III -dt & -3290.562792570 & 0.9 & -3290.429966570 & -23.2 \\
3 & INT-IV'-dt-R & -3290.542048490 & 13.9 & -3290.409620490 & -10.4 \\
4 & INT-IV'-dt-S & -3290.542212860 & 13.8 & -3290.408751860 & -9.9 \\
5 & INT-IV-dt-R & -3290.544400380 & 12.4 & -3290.410967380 & -11.3 \\
6 & INT-IV-dt-S & -3290.544906930 & 12.1 & -3290.410545930 & -11.0 \\
7 & TS-III'-dt-R & -3290.533175860 & 19.5 & -3290.403648860 & -6.7 \\
8 & TS-III'-dt-S & -3290.533615360 & 19.2 & -3290.402608360 & -6.0 \\
9 & TS-III-dt-R & -3290.526556620 & 23.6 & -3290.397743620 & -3.0 \\
10 & TS-III-dt-S & -3290.537422050 & 16.8 & -3290.406708050 & -8.6 \\
\hline
\end{tabular}

dt refers to direct proton transfer from the N-H of indole.

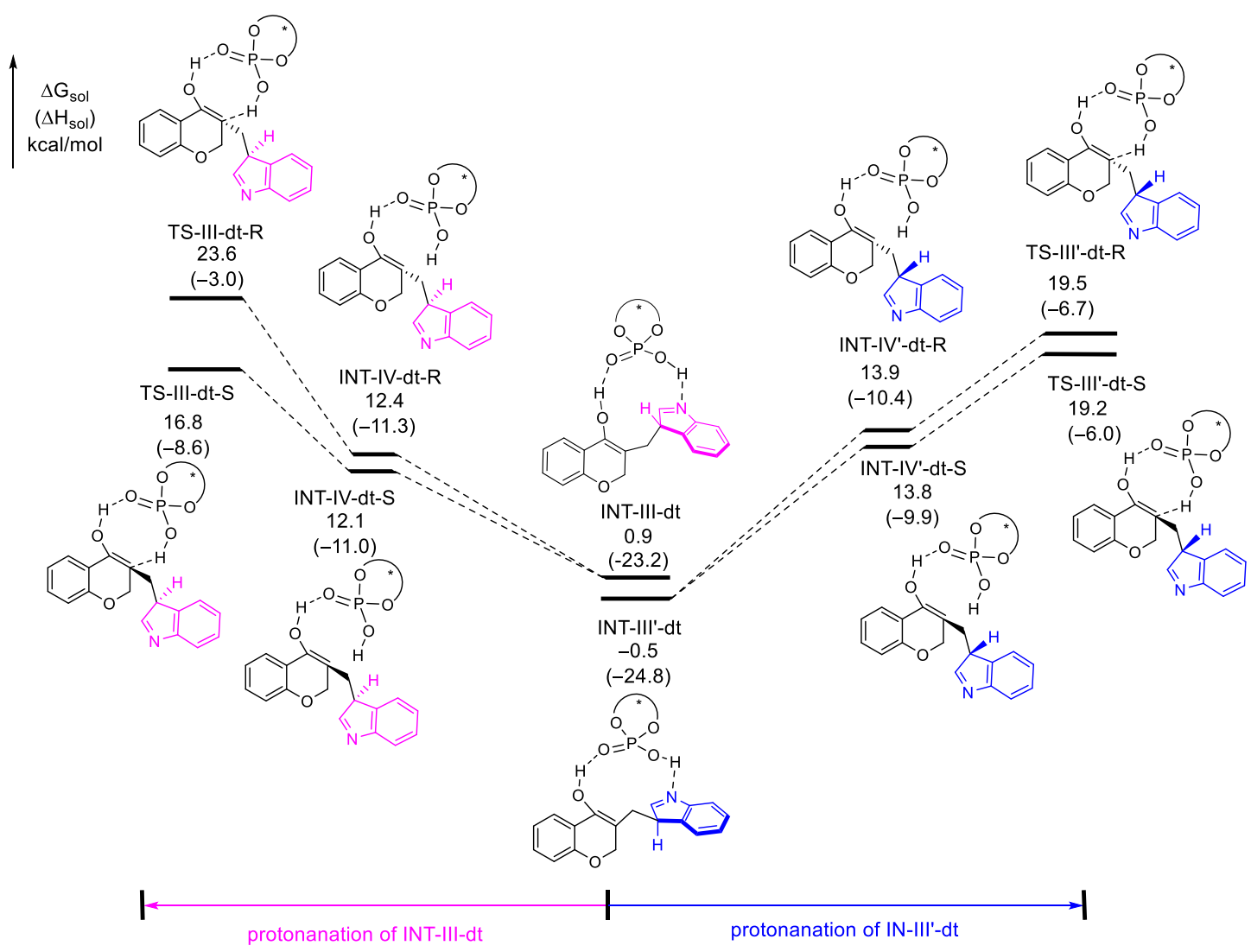

Figure S3. The computed energy surfaces for transition states with the direct proton transfer from the indole N-H to SPA, proceeding with the [1,3]-proton transfer of enol moiety. INT-III-dt and IN-III'-dt derived from INT-II and INT-II' (see Figure S1), respectively. 
Note: In this pathway, enol protonation takes place before indole re-aromatization. A temporary C-3 stereocenter of indole is build up which might influence the protonation. However, much higher energy barriers are observed in the enantioselective protonation step (Figure S1 vs Figure S3). 
Table S7. Sum of computed energies of stationary points (For Figure S4)

\begin{tabular}{cccccc}
\hline entry & Structure & $\Delta \mathrm{G}_{\text {sol }}$ (a.u.) & $\begin{array}{c}\Delta \Delta \mathrm{G}_{\text {sol }} \\
(\mathrm{kcal} / \mathrm{mol})\end{array}$ & $\Delta \mathrm{H}_{\text {sol }}$ (a.u. $)$ & $\begin{array}{c}\Delta \Delta \mathrm{H}_{\text {sol }} \\
(\mathrm{kcal} / \mathrm{mol})\end{array}$ \\
\hline 1 & Enol & -900.2287096220 & N/A & -900.1780096220 & N/A \\
2 & TS-S1 & -900.1415953930 & 54.7 & -900.0916173930 & 54.2 \\
3 & TS-S2 & -1800.413237450 & 27.7 & -1800.329568450 & 16.6 \\
4 & TS-H $\mathbf{O}$ & -976.634446094 & 33.3 & -976.581510094 & 23.3 \\
5 & $\mathbf{H}_{2} \mathbf{O}$ & -76.4587871170 & N/A & -76.4407031170 & N/A \\
\hline
\end{tabular}

(A)

$\uparrow \begin{gathered}\Delta \mathrm{G}_{\mathrm{sol}} \\ \left(\Delta \mathrm{H}_{\mathrm{sol}}\right) \\ \mathrm{kcal} / \mathrm{mol}\end{gathered}$
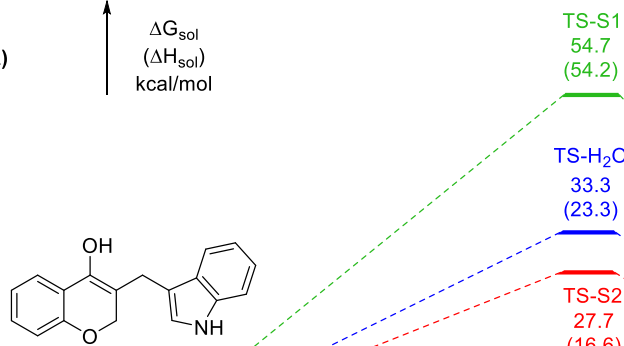

TS- $\mathrm{H}_{2} \mathrm{O}$

(B)
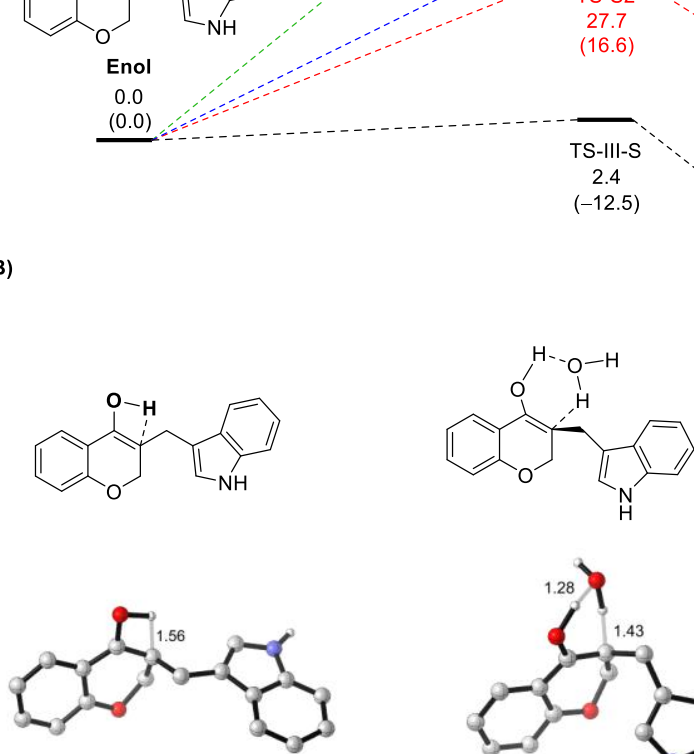

TS-S1
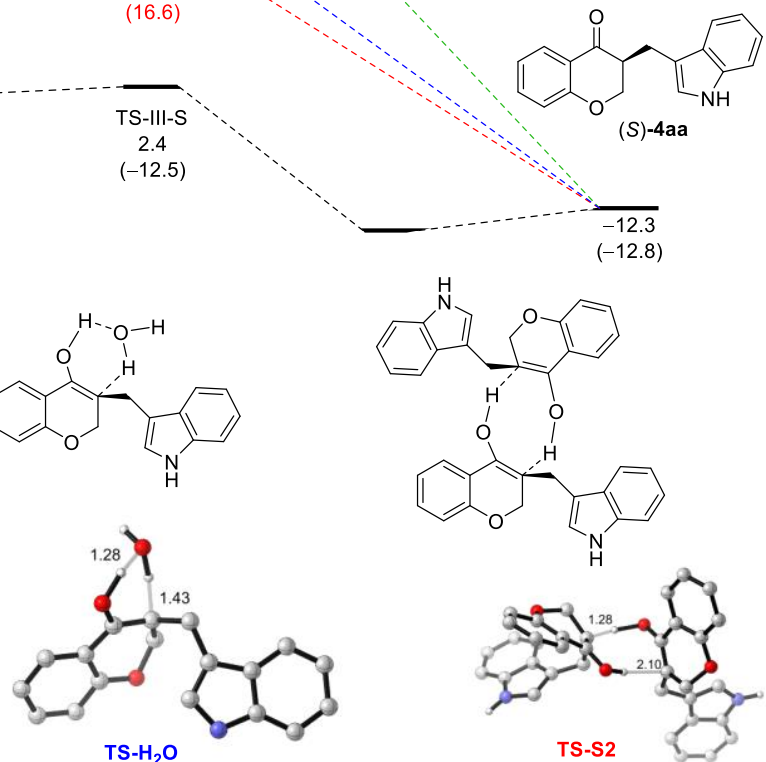

Figure S4. The computed energy surfaces for [1,3]-proton transfer transition state promoted by other species. $\Delta \Delta \mathrm{G}_{\text {sol }}$ was calculated based on using Enol as zero energy point. For TS-S1 (green line): [1,3]-proton transfer spontaneously by Enol itself; for TS- $\mathbf{H}_{2} \mathrm{O}$ (blue line): [1,3]-proton transfer assisted by trace $\mathrm{H}_{2} \mathrm{O}$; for TS-S2 (red line): [1,3]-proton transfer undergoes intermolecularly by another Enol; for TS-III-S (black line): [1,3]-proton transfer assisted by $(S)-\mathbf{3 g}$. 
Table S8. Sum of computed energies of stationary points (For Figure S5\&S6)

\begin{tabular}{cccccc}
\hline entry & Structure & $\Delta \mathrm{G}_{\text {sol }}$ (a.u.) & $\begin{array}{c}\Delta \Delta \mathrm{G}_{\text {sol }} \\
(\mathrm{kcal} / \mathrm{mol})\end{array}$ & $\Delta \mathrm{H}_{\text {sol }}($ a.u. $)$ & $\begin{array}{c}\Delta \Delta \mathrm{H}_{\text {sol }} \\
(\mathrm{kcal} / \mathrm{mol})\end{array}$ \\
\hline 1 & 2b & -403.153846513 & $\mathrm{~N} / \mathrm{A}$ & -403.119455513 & $\mathrm{~N} / \mathrm{A}$ \\
2 & INT-I'-Me & -3329.865344810 & -1.7 & -3329.724355810 & -22.8 \\
3 & INT-I-Me & -3329.865084430 & -1.6 & -3329.723458430 & -22.3 \\
4 & TS-I'-Me & -3329.843529670 & 12.0 & -3329.709257670 & -13.3 \\
5 & TS-I-Me & -3329.846030060 & 10.4 & -3329.710638060 & -14.2 \\
6 & TS-III-S (4ab) & -3329.85619361 & $\mathrm{~N} / \mathrm{A}$ & -3329.72162461 & $\mathrm{~N} / \mathrm{A}$ \\
7 & TS-III-R (4ab) & -3329.85118488 & 3.14 & -3329.71691888 & 2.95 \\
8 & TS-III-S (4ad) & -3329.86862890 & N/A & -3329.73510490 & N/A \\
9 & TS-III-R (4ad) & -3329.86555781 & 2.07 & -3329.73221881 & 1.81 \\
\hline
\end{tabular}

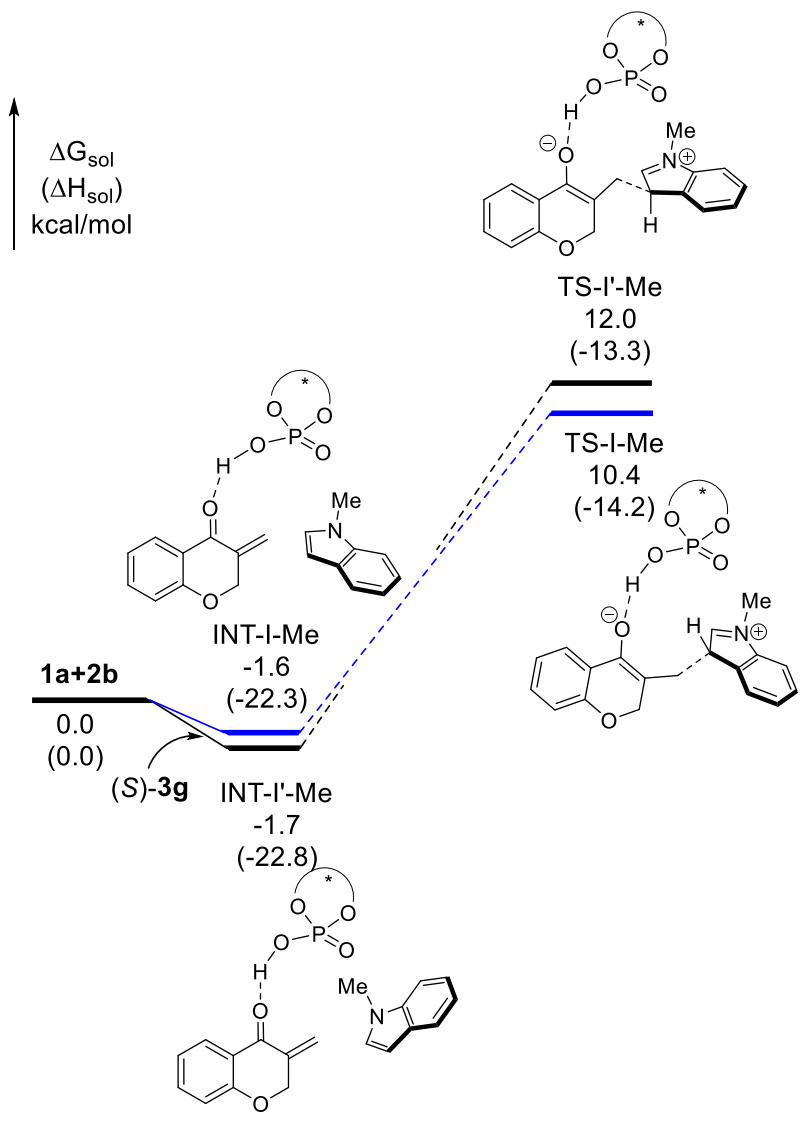

Figure S5. The conjugate step of enone 1a with $N$-methyl indole $\mathbf{2 b}$. 
(a) proton transfer transition sates of $4 \mathrm{ab}(\Delta \Delta \mathrm{G}=3.14 \mathrm{kcal} / \mathrm{mol})$

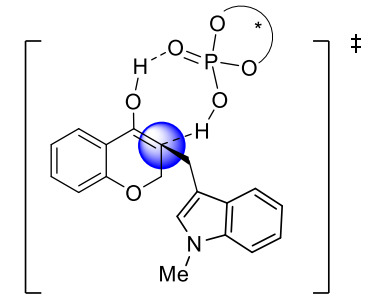

TS-III-S (4ab)

0.0

(using as zero-point energy)

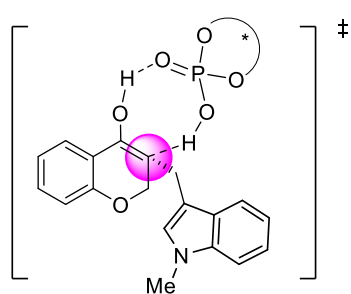

TS-III-R (4ab)

3.14

(b) proton transfer transition sates of $4 \mathrm{ad}(\Delta \Delta \mathrm{G}=2.07 \mathrm{kcal} / \mathrm{mol})$

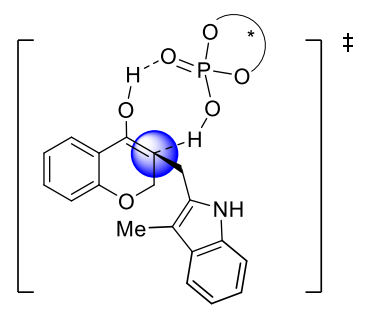

TS-III-S (4ad)

0.0

(using as zero-point energy)

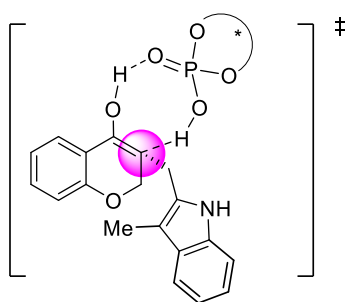

TS-III-R (4ad)

2.07

Figure S6. The transition states of enantioselective proton transfer of $4 \mathbf{a b}$ and $4 \mathbf{a d .}$ 
Table S9. Sum of computed energies of stationary points (For Figure S7)

\begin{tabular}{cccccc}
\hline entry & Structure & $\Delta \mathrm{G}_{\text {sol }}$ (a.u.) & $\begin{array}{c}\Delta \Delta \mathrm{G}_{\text {sol }} \\
(\mathrm{kcal} / \mathrm{mol})\end{array}$ & $\Delta \mathrm{H}_{\text {sol }}$ (a.u.) & $\begin{array}{c}\Delta \Delta \mathrm{H}_{\text {sol }} \\
(\mathrm{kcal} / \mathrm{mol})\end{array}$ \\
\hline 1 & TS-III-R2 & -3290.553137400 & 7.0 & -3290.422013400 & -18.2 \\
2 & TS-III-R3 & -3290.552593070 & 7.3 & -3290.421329070 & -17.8 \\
3 & TS-III-R4 & -3290.549908750 & 9.0 & -3290.419735750 & -16.8 \\
4 & TS-III-S2 & -3290.556016210 & 5.1 & -3290.424849210 & -20.0 \\
\hline
\end{tabular}

a. other representative TS-III-R conformers

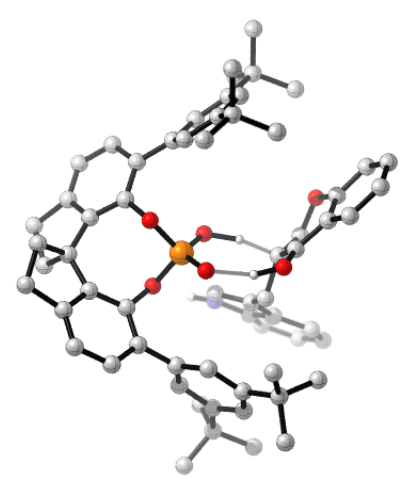

TS-III-R2

$\Delta \Delta \mathrm{G}=7.0 \mathrm{kcal} / \mathrm{mol}$

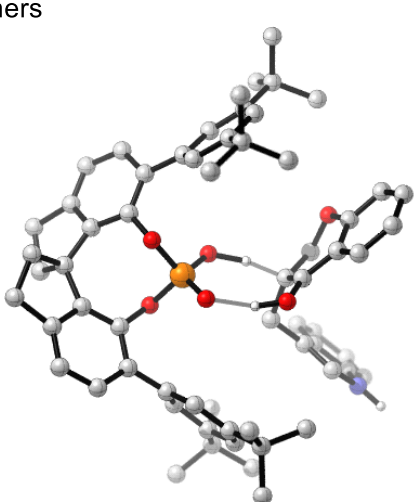

TS-III-R3

$\Delta \Delta \mathrm{G}=7.3 \mathrm{kcal} / \mathrm{mol}$

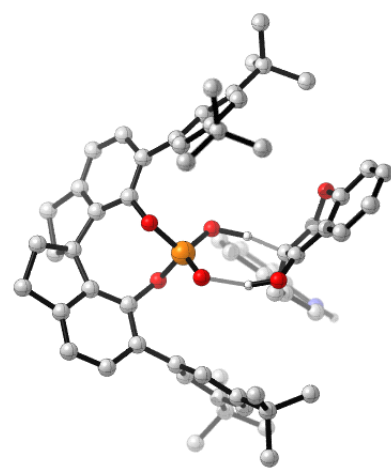

TS-III-R4 $\Delta \Delta \mathrm{G}=9.0 \mathrm{kcal} / \mathrm{mol}$

b. other representative TS-III-S conformers

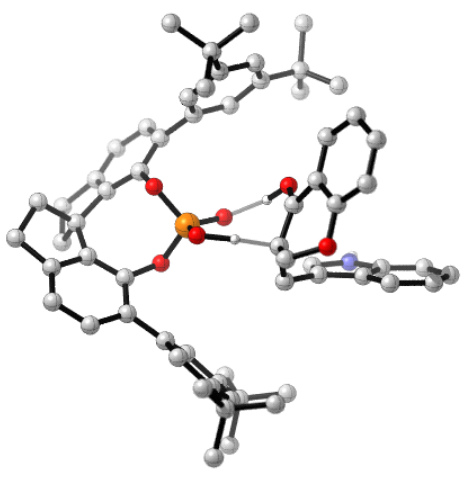

TS-III-S2

$\Delta \Delta \mathrm{G}=5.1 \mathrm{kcal} / \mathrm{mol}$

Figure S7. Other representative conformers of TS-III-R and TS-III-S. Trivial H atoms are omitted for clarity. 
Table S10. Sum of computed energies of stationary points (For Figure S8)

\begin{tabular}{cccccc}
\hline Structure & E (a.u.) & Structure & E (a.u.) & Structure & E (a.u.) \\
\hline $\mathbf{A}$ & -2391.169508 & $\mathbf{A}(\mathbf{S})$ & -2391.126707 & $\mathbf{A ( R )}$ & -2391.119725 \\
$\mathbf{B}$ & -900.4809945 & $\mathbf{B}(\mathbf{S})$ & -900.4597632 & $\mathbf{B ( R )}$ & -900.4566742 \\
\hline
\end{tabular}

(a) Distortion/interaction analysis of TS-III-S and TS-III-R
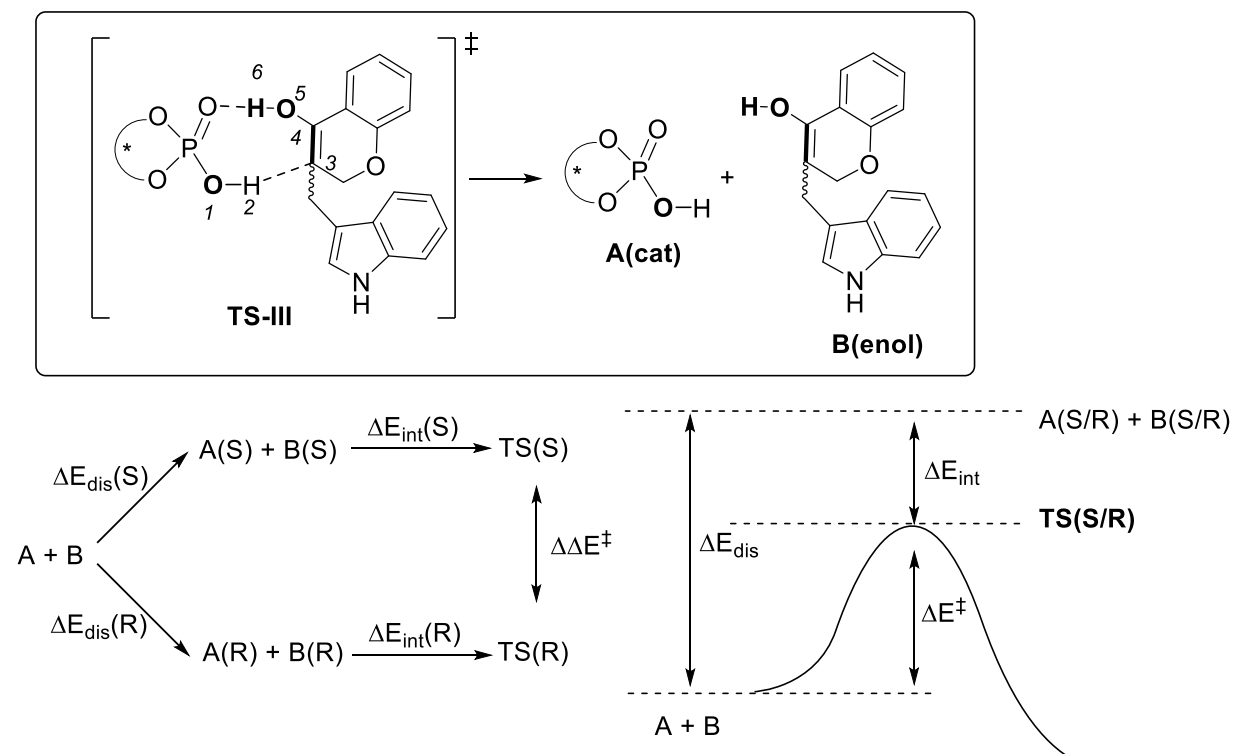

\begin{tabular}{c|c|c|c|c|c}
\hline & $\Delta \mathrm{E}_{\mathrm{dis}}$ & $\Delta \mathrm{E}_{\mathrm{dis}}($ enol $)$ & $\Delta \mathrm{E}_{\mathrm{dis}}($ cat $)$ & $\Delta \mathrm{E}_{\mathrm{int}}$ & $\Delta \mathrm{E}^{\ddagger}$ \\
\hline TS-III-S & 40.2 & 13.3 & 26.9 & -51.0 & -10.8 \\
\hline TS-III-R & 46.5 & 15.2 & 31.3 & -54.1 & -7.6 \\
\hline & $\Delta \Delta \mathrm{E}_{\mathrm{dis}}$ & $\Delta \Delta \mathrm{E}_{\mathrm{dis}}(\mathrm{enol})$ & $\Delta \Delta \mathrm{E}_{\mathrm{dis}}(\mathrm{cat})$ & $\Delta \Delta \mathrm{E}_{\mathrm{int}}$ & $\Delta \Delta \mathrm{E}^{\ddagger}$ \\
\cline { 2 - 6 } & 6.3 & 1.9 & 4.4 & -3.1 & 3.2 \\
\hline
\end{tabular}

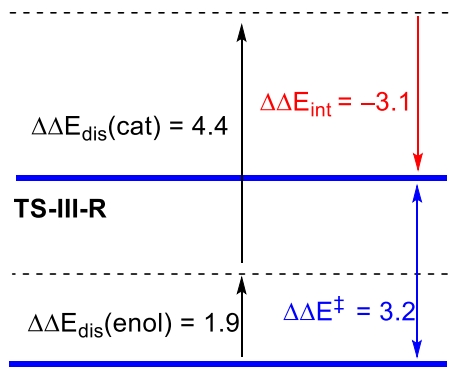

TS-III-S

(b) Natural bond orbital (NBO) analysis of TS-III-S and TS-III-R

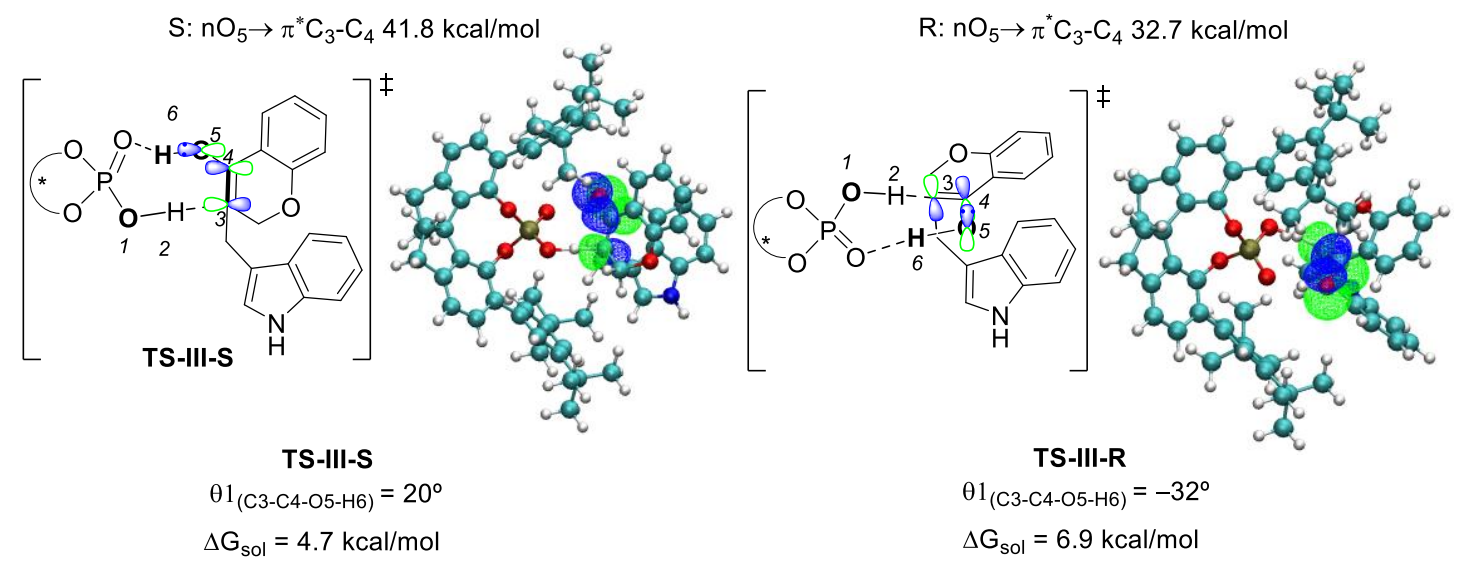

Figure S8. (a) Distortion/interaction analysis of TS-III-S and TS-III-R; (b) NBO analysis of TS-III-S and TS-III-R. 
The Cartesian coordinates $(\AA ̊)$ and energies at $258 \mathrm{~K}$ for the optimized structures.

$1 \mathbf{a}$ (Table S4, entry 1)

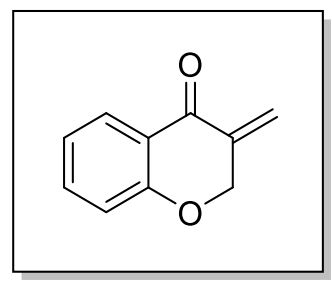

SCF energy [B3LYP-GD3BJ/6-311+G**/SMD (Chlorobenzene)]: -536.504278954 a.u. Thermal correction to Gibbs free energy at $258 \mathrm{~K}: 0.127339000$ a.u.

Gibbs free energy at $258 \mathrm{~K}$ [B3LYP-GD3BJ/6-311+G**/SMD (Chlorobenzene)]: -536.376939954 a.u.

$\begin{array}{crrr}01 & & & \\ \text { C } & 1.55625100 & -1.49010700 & 0.43845100 \\ \text { C } & -0.67309100 & -0.86672500 & -0.06761500 \\ \text { C } & -0.36718900 & 0.50347600 & 0.06659700 \\ \text { C } & 1.03926500 & 0.96593200 & 0.04352300 \\ \text { C } & 2.05868300 & -0.13387200 & 0.01867900 \\ \text { H } & -2.21262300 & -2.34599400 & -0.27173700 \\ \text { H } & 1.43934700 & -1.52030000 & 1.53342000 \\ \text { C } & -2.00592800 & -1.28622400 & -0.16254700 \\ \text { C } & -1.41864900 & 1.43504800 & 0.12149600 \\ \text { C } & -2.73977400 & 1.02150200 & 0.04457100 \\ \text { C } & -3.02661600 & -0.34452800 & -0.10548800 \\ \text { H } & -1.15471800 & 2.48409600 & 0.21309100 \\ \text { H } & -3.54651500 & 1.74671400 & 0.09157900 \\ \text { H } & -4.05946500 & -0.67619900 & -0.17265000 \\ \text { O } & 1.34867400 & 2.15119200 & 0.00438700 \\ \text { O } & 0.28692500 & -1.83006700 & -0.14290700 \\ \text { C } & 3.32646100 & 0.12118500 & -0.31755600 \\ \text { H } & 4.08074800 & -0.66007900 & -0.36458000 \\ \text { H } & 3.63190000 & 1.13781100 & -0.54594700 \\ \text { H } & 2.24005500 & -2.28917000 & 0.14431400\end{array}$


2a (Table S4, entry 2)

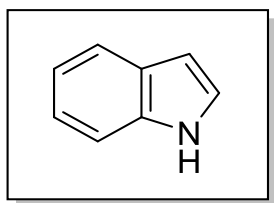

SCF energy [B3LYP-GD3BJ/6-311+G**/SMD (Chlorobenzene)]: -363.959993874 a.u.

Thermal correction to Gibbs free energy at $258 \mathrm{~K}: 0.104531000$ a.u.

Gibbs free energy at $258 \mathrm{~K}$ [B3LYP-GD3BJ/6-311+G**/SMD (Chlorobenzene)]:

-363.855462874 a.u.

$\begin{array}{crrr}01 & & & \\ \mathrm{C} & 0.25009300 & 0.75204500 & 0.00000000 \\ \mathrm{C} & 0.24798100 & -0.67204200 & 0.00000000 \\ \mathrm{C} & -0.93518000 & -1.41876900 & 0.00000000 \\ \mathrm{C} & -2.13585500 & -0.71873500 & 0.00000000 \\ \mathrm{C} & -2.15926600 & 0.69169300 & 0.00000000 \\ \mathrm{C} & -0.98259000 & 1.42896500 & 0.00000000 \\ \mathrm{C} & 1.62596200 & 1.16693300 & 0.00000100 \\ \mathrm{C} & 2.39016000 & 0.03014900 & -0.00000100 \\ \mathrm{H} & -0.91798100 & -2.50577900 & 0.00000000 \\ \mathrm{H} & -3.07317000 & -1.26833400 & 0.00000000 \\ \mathrm{H} & -3.11632100 & 1.20627800 & 0.00000000 \\ \mathrm{H} & -1.01144300 & 2.51568000 & 0.00000000 \\ \mathrm{H} & 1.99879900 & 2.18197900 & 0.00000300 \\ \mathrm{H} & 3.46507400 & -0.08619800 & -0.00000300 \\ \mathrm{~N} & 1.56662800 & -1.08093700 & 0.00000000 \\ \mathrm{H} & 1.88082500 & -2.03850600 & 0.00000000\end{array}$


(S)-3g (Table S4, entry 3)

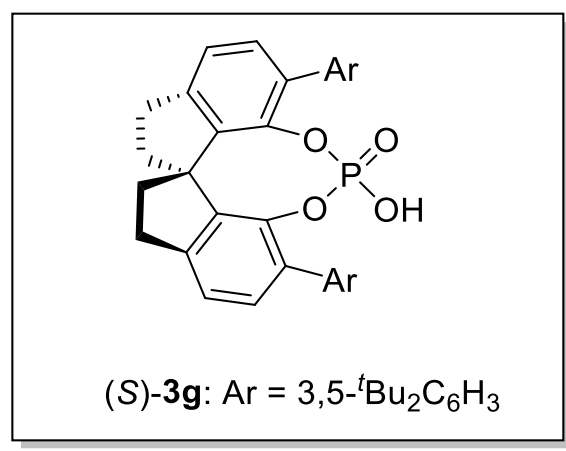

SCF energy [B3LYP-GD3BJ/6-311+G**/SMD (Chlorobenzene)]: -2391.169507900 a.u.

Thermal correction to Gibbs free energy at $258 \mathrm{~K}: 0.837696000$ a.u.

Gibbs free energy at $258 \mathrm{~K}$ [B3LYP-GD3BJ/6-311+G**/SMD (Chlorobenzene)]: -2390.331811900 a.u.

\begin{tabular}{lrrr}
01 & & & \\
$\mathrm{P}$ & -0.04397300 & 0.14601500 & -0.20128800 \\
$\mathrm{O}$ & 0.97761000 & 1.07730600 & 0.65092100 \\
$\mathrm{O}$ & -1.13780500 & 1.19494800 & -0.81055200 \\
$\mathrm{O}$ & -0.73962500 & -0.55639500 & 1.06611900 \\
0 & 0.49382100 & -0.70071600 & -1.27838600 \\
$\mathrm{C}$ & 1.76297300 & 1.96012200 & -0.11051200 \\
$\mathrm{C}$ & 3.03341300 & 1.54688500 & -0.55559800 \\
$\mathrm{C}$ & 3.73001800 & 2.41594500 & -1.41215200 \\
$\mathrm{C}$ & 3.18306300 & 3.62564700 & -1.83820300 \\
$\mathrm{C}$ & 1.93565600 & 4.01310600 & -1.36016400 \\
$\mathrm{C}$ & 1.12750000 & 5.24389500 & -1.70939100 \\
$\mathrm{C}$ & -0.28115300 & 4.87324400 & -1.19586600 \\
$\mathrm{C}$ & -0.01997600 & 3.93211900 & 0.02712300 \\
$\mathrm{C}$ & 1.23333700 & 3.20231000 & -0.45445600 \\
$\mathrm{C}$ & 0.27769300 & 4.79441600 & 1.30143500 \\
$\mathrm{C}$ & -1.11358800 & 5.17255700 & 1.85473600 \\
$\mathrm{C}$ & -1.95511100 & 3.98123000 & 1.45307600 \\
$\mathrm{C}$ & -3.19238500 & 3.58080100 & 1.94743900 \\
$\mathrm{C}$ & -3.76047800 & 2.39450700 & 1.48281400 \\
$\mathrm{C}$ & -3.10051700 & 1.57671300 & 0.55173700 \\
$\mathrm{C}$ & -1.85282400 & 2.01613900 & 0.06532800 \\
$\mathrm{C}$ & -1.29221100 & 3.22033300 & 0.47710700 \\
$\mathrm{H}$ & -1.56791600 & -1.00831900 & 0.82116000 \\
$\mathrm{H}$ & 4.71551900 & 2.11752200 & -1.75735200 \\
$\mathrm{H}$ & 3.72906700 & 4.25467900 & -2.53694300 \\
$\mathrm{H}$ & 1.51639400 & 6.13683100 & -1.19978000 \\
& & & \\
\hline
\end{tabular}




\begin{tabular}{|c|c|c|c|}
\hline $\mathrm{H}$ & 1.14082200 & 5. 46394600 & -2.78291900 \\
\hline $\mathrm{H}$ & -0.89127200 & 5. 74285900 & -0.93137700 \\
\hline $\mathrm{H}$ & -0.81870100 & 4. 30869900 & -1.96614400 \\
\hline $\mathrm{H}$ & 0.90887800 & 5. 66107000 & 1. 08161200 \\
\hline $\mathrm{H}$ & 0.80763000 & 4. 17215000 & 2. 03144800 \\
\hline $\mathrm{H}$ & -1.48836200 & 6. 09845900 & 1. 39581900 \\
\hline $\mathrm{H}$ & -1.10810900 & 5. 33779500 & 2. 93805200 \\
\hline $\mathrm{H}$ & -3.70849900 & 4. 17462800 & 2. 69762200 \\
\hline $\mathrm{H}$ & -4.72954300 & 2. 07685100 & 1. 85691500 \\
\hline $\mathrm{C}$ & -3.67983200 & 0.26179100 & 0.15744400 \\
\hline $\mathrm{C}$ & -4.16789100 & -0.60583500 & 1. 14949300 \\
\hline $\mathrm{C}$ & -3.74726500 & -0.13435400 & -1.18732400 \\
\hline $\mathrm{C}$ & -4.72785700 & -1.84842300 & 0.82235100 \\
\hline $\mathrm{C}$ & -4.29150100 & -1.36961700 & -1.55487900 \\
\hline $\mathrm{C}$ & -4.77568100 & -2.20110100 & -0.53363200 \\
\hline $\mathrm{C}$ & 3. 63794700 & 0.24454400 & -0.16444100 \\
\hline $\mathrm{C}$ & 3. 66669600 & -0.16303100 & 1. 17633600 \\
\hline $\mathrm{C}$ & 4. 22595000 & -0.56979200 & -1.14118900 \\
\hline $\mathrm{C}$ & 4. 27509400 & -1.36394000 & 1. 55494500 \\
\hline $\mathrm{C}$ & 4. 84285000 & -1.77934200 & -0.80487200 \\
\hline C & 4. 85699800 & -2.14702500 & 0.54762800 \\
\hline $\mathrm{H}$ & -4.09117400 & -0.29417800 & 2. 18493500 \\
\hline $\mathrm{H}$ & -3.36255300 & 0.53902300 & -1.94150500 \\
\hline $\mathrm{H}$ & -5.19961800 & -3.16322800 & -0.80545800 \\
\hline $\mathrm{H}$ & 3. 20949800 & 0.47686600 & 1. 92010500 \\
\hline $\mathrm{H}$ & 4. 16400300 & -0.25421400 & -2.17577600 \\
\hline $\mathrm{H}$ & 5. 33147800 & -3.08318700 & 0.82734100 \\
\hline $\mathrm{C}$ & 4. 31973600 & -1.84490100 & 3. 01857300 \\
\hline $\mathrm{C}$ & 3. 62884500 & -0.86018400 & 3. 98134800 \\
\hline $\mathrm{H}$ & 2. 57102100 & -0.72138700 & 3. 73212100 \\
\hline $\mathrm{H}$ & 4. 11313600 & 0.12311900 & 3. 97662200 \\
\hline $\mathrm{H}$ & 3. 68100000 & -1.24832300 & 5. 00516600 \\
\hline $\mathrm{C}$ & 3. 59791100 & -3.20887000 & 3. 13207700 \\
\hline $\mathrm{H}$ & 4. 06742300 & -3.97278200 & 2. 50347900 \\
\hline $\mathrm{H}$ & 2. 54975600 & -3.12189300 & 2. 82467200 \\
\hline $\mathrm{H}$ & 3. 62179800 & -3.56858500 & 4. 16849400 \\
\hline $\mathrm{C}$ & 5. 79142100 & -2.00437900 & 3. 46824700 \\
\hline $\mathrm{H}$ & 6. 33194400 & -2.72790700 & 2. 84889200 \\
\hline $\mathrm{H}$ & 5. 83832000 & -2.35394200 & 4. 50713200 \\
\hline $\mathrm{H}$ & 6. 32493100 & -1.04880100 & 3. 40695800 \\
\hline $\mathrm{C}$ & 5. 48262400 & -2.70584100 & -1.85704300 \\
\hline $\mathrm{C}$ & 5. 34700800 & -2.15072700 & -3.28769800 \\
\hline $\mathrm{H}$ & 5. 84476000 & -1.18050100 & -3.39851200 \\
\hline $\mathrm{H}$ & 4. 29777900 & -2.03304500 & -3.58021700 \\
\hline
\end{tabular}


$-3.99739600$

$4.79090600-4.08930000$

$-1.81549800$

$4.89580200-4.56981800$

$-0.83699900$

5. 23246400

$-4.75911700$

$-2.56404700$

3. 72022300

$-3.99626600$

$-2.02855900$

6. 98895600

$-2.87527800$

$-1.54782200$

7. 45753600

$-3.54359600$

$-2.28106100$

7. 15628500

$-3.30215900$

$-0.55332100$

7. 50768700

$-1.91033100$

$-1.58779700$

$-4.35594900$

$-1.84008000$

$-3.02079700$

-3. 79240300

$-0.79089700$

$-3.99835500$

$-2.73823500$

$-0.57085600$

$-3.79787100$

$-4.35495300$

0. 14897800

$-3.95709800$

$-3.86183200$

$-1.17142200$

$-5.02366200$

$-3.52261700$

-3. 13503200

-3. 17549400

$-3.89597200$

$-3.94045600$

$-2.53377600$

$-2.47266800$

$-2.95770900$

$-2.91693800$

$-3.56209100$

$-3.49035100$

$-4.21251400$

$-5.82588100$

$-2.12267500$

$-3.41139100$

$-6.27754300$

$-2.89536800$

$-2.78027400$

$-5.88233200$

$-2.46819800$

-4. 45073600

-6. 43716200

$-1.21735900$

$-3.32083300$

$-5.27590800$

$-2.81830100$

1. 88750500

$-5.11607600$

$-2.27023500$

3. 31843400

$-5.66109300$

$-1.32995500$

3. 45925700

$-4.06468600$

$-2.09867500$

3. 57576100

$-5.51718800$

$-2.99381000$

4. 03679900

$-4.51872100$

$-4.16507400$

1. 80335900

$-4.62903200$

$-4.63766600$

0. 82182800

$-4.90369200$

$-4.86544000$

2. 55450200

$-3.44719900$

$-4.02639000$

1. 98831100

$-6.78248500$

$-3.06025900$

1. 63088100

$-7.18884000$

-3. 75751200

2. 37370800

$-6.96425700$

$-3.48654600$

0. 63887100

$-7.34667100$

$-2.12313100$

1. 69994500 
INT-I' (Table S4, entry 4)

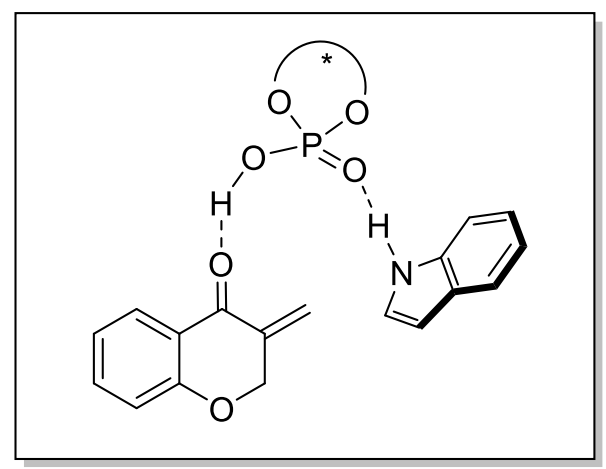

SCF energy [B3LYP-GD3BJ/6-311+G**/SMD (Chlorobenzene)]: -3291.684336500 a.u.

Thermal correction to Gibbs free energy at $258 \mathrm{~K}: 1.108647000$ a.u.

Gibbs free energy at $258 \mathrm{~K}$ [B3LYP-GD3BJ/6-311+G**/SMD (Chlorobenzene)]: -3290.575689500 a.u.

\begin{tabular}{lrrr}
$0 \mathrm{H}$ & & & \\
$\mathrm{H}$ & -0.94602300 & -0.37941400 & 0.95370200 \\
$\mathrm{C}$ & 1.79396000 & -2.83124100 & -1.89876200 \\
$\mathrm{C}$ & 1.66675900 & -3.94682800 & -2.78227200 \\
$\mathrm{C}$ & 2.40873800 & -5.11202000 & -2.51150300 \\
$\mathrm{C}$ & 3.23698100 & -5.14568400 & -1.39548800 \\
$\mathrm{C}$ & 3.34416300 & -4.03164900 & -0.53304900 \\
$\mathrm{C}$ & 2.62731900 & -2.86331800 & -0.77209200 \\
$\mathrm{C}$ & 0.35301700 & -2.26778800 & -3.51478800 \\
$\mathrm{C}$ & 0.73838300 & -3.55589800 & -3.80275900 \\
$\mathrm{H}$ & 3.81678400 & -6.04026100 & -1.18253700 \\
$\mathrm{H}$ & 3.99919200 & -4.08715300 & 0.33258800 \\
$\mathrm{H}$ & 2.71889600 & -2.00026300 & -0.11907800 \\
$\mathrm{H}$ & 0.83964300 & -0.91453600 & -1.93840200 \\
$\mathrm{H}$ & -0.32230300 & -1.61164600 & -4.04676000 \\
$\mathrm{H}$ & 0.40972800 & -4.14654600 & -4.64721000 \\
$\mathrm{~N}$ & 0.98474700 & -1.82625600 & -2.37559700 \\
$\mathrm{C}$ & -0.24918000 & -5.46911600 & 0.32038300 \\
$\mathrm{C}$ & 0.08736100 & -4.68995600 & 2.53574300 \\
$\mathrm{C}$ & -0.14022500 & -3.34763100 & 2.14915700 \\
$\mathrm{C}$ & -0.80656900 & -3.06357500 & 0.86994900 \\
$\mathrm{C}$ & -1.06629700 & -4.24716500 & 0.00451000 \\
$\mathrm{H}$ & 0.74400300 & -6.01827200 & 4.08434800 \\
$\mathrm{H}$ & 0.78031300 & -5.33830700 & -0.03970900 \\
$\mathrm{C}$ & 0.58779600 & -4.97934300 & 3.81314000 \\
$\mathrm{C}$ & 0.17279900 & -2.31572800 & 3.05813900 \\
$\mathrm{C}$ & 0.68710800 & -2.60363800 & 4.31145500 \\
& & & \\
\hline & & &
\end{tabular}




\begin{tabular}{|c|c|c|c|}
\hline C & 0.88435500 & -3.94313500 & 4. 68708700 \\
\hline H & -0.00325800 & -1.28768400 & 2. 76025700 \\
\hline H & 0.92900600 & -1.80080400 & 5. 00071300 \\
\hline H & 1. 28098600 & -4.17544500 & 5. 67191100 \\
\hline 0 & -1.19736500 & -1.93807200 & 0.53230600 \\
\hline 0 & -0.18744200 & -5.74625800 & 1. 73517900 \\
\hline C & -1.93742400 & -4.16777100 & -1.00811500 \\
\hline H & -2.15126500 & -5.02189400 & -1.64451300 \\
\hline $\mathrm{H}$ & -2.44179300 & -3.23218000 & -1.22622900 \\
\hline H & -0.66751700 & -6.36812100 & -0.13579800 \\
\hline $\mathrm{P}$ & -0.20507200 & 1. 43271400 & -0.02415000 \\
\hline 0 & -1.44663700 & 2. 31882000 & -0.59886900 \\
\hline 0 & 0.69386200 & 2. 49407200 & 0.81614100 \\
\hline 0 & 0.46006300 & 0.73673000 & -1.14890800 \\
\hline 0 & -0.73875500 & 0.57332200 & 1. 19179300 \\
\hline $\mathrm{C}$ & -2.29862800 & 2. 95946700 & 0.31130800 \\
\hline C & -3.49833600 & 2. 32844100 & 0.69725900 \\
\hline C & -4.26454400 & 2. 95544200 & 1. 69491000 \\
\hline C & -3.85384600 & 4. 13888300 & 2. 30716100 \\
\hline $\mathrm{C}$ & -2.68342200 & 4. 75347500 & 1. 87604900 \\
\hline $\mathrm{C}$ & -2.02921600 & 6. 01715100 & 2. 39081900 \\
\hline C & -0.61119400 & 5. 92930200 & 1. 78516200 \\
\hline C & -0.80194000 & 5. 14717300 & 0.44243800 \\
\hline C & -1.92140200 & 4. 19085800 & 0.84005200 \\
\hline $\mathrm{C}$ & -1.27337400 & 6. 13368100 & -0.67971200 \\
\hline C & 0.02607800 & 6. 78337500 & -1.20213000 \\
\hline C & 1. 04488600 & 5. 68525200 & -0.99110800 \\
\hline C & 2. 31712900 & 5. 55943700 & -1.53790800 \\
\hline C & 3. 07958900 & 4. 43394900 & -1.23044700 \\
\hline $\mathrm{C}$ & 2. 58233600 & 3. 39212500 & -0.42798000 \\
\hline C & 1. 27985600 & 3. 54153900 & 0.08796000 \\
\hline C & 0.53396200 & 4. 69527500 & -0.13776100 \\
\hline H & -5.20034900 & 2. 49223400 & 1. 99304900 \\
\hline H & -4.44987700 & 4. 57546500 & 3. 10477000 \\
\hline H & -2.55990100 & 6. 91286300 & 2. 03828800 \\
\hline H & -2.01862500 & 6. 06907800 & 3. 48556200 \\
\hline H & -0.13975500 & 6.90560400 & 1. 63491400 \\
\hline H & 0.03769900 & 5. 34292500 & 2. 44550900 \\
\hline H & -2.00836000 & 6. 85925900 & -0.31715100 \\
\hline H & -1.74400500 & 5. 55347000 & -1.48150400 \\
\hline H & 0.28342800 & 7. 68357300 & -0.62587100 \\
\hline H & -0.04802000 & 7. 09242100 & -2.25102400 \\
\hline H & 2. 71820000 & 6. 32717300 & -2.19497000 \\
\hline $\mathrm{H}$ & 4. 08764700 & 4. 34300600 & -1.62361100 \\
\hline
\end{tabular}




\begin{tabular}{|c|c|c|c|}
\hline C & 3. 43822000 & 2. 20467500 & -0.15880100 \\
\hline $\mathrm{C}$ & 4. 16550700 & 1. 62256000 & -1.20037100 \\
\hline $\mathrm{C}$ & 3. 59030900 & 1. 70012100 & 1. 14430500 \\
\hline $\mathrm{C}$ & 5. 04479600 & 0.55207100 & -0.97788600 \\
\hline $\mathrm{C}$ & 4. 46081600 & 0.64232300 & 1. 41141500 \\
\hline $\mathrm{C}$ & 5. 17513900 & 0.08586700 & 0.33361600 \\
\hline $\mathrm{C}$ & -3.98445000 & 1. 06169400 & 0.08427800 \\
\hline C & -3.97155600 & 0.88496600 & -1.31085100 \\
\hline C & -4.54393400 & 0.06255700 & 0.88595300 \\
\hline $\mathrm{C}$ & -4.51800600 & -0.25413000 & -1.90255300 \\
\hline $\mathrm{C}$ & -5.10696800 & -1.09784900 & 0.33241100 \\
\hline $\mathrm{C}$ & -5.08408800 & -1.22879100 & -1.05918400 \\
\hline H & 5. 85326000 & -0.73513400 & 0.53381700 \\
\hline $\mathrm{H}$ & 4. 02408900 & 2. 00513100 & -2.20632500 \\
\hline H & 3. 02690500 & 2.16253600 & 1. 94451400 \\
\hline H & -5.52361100 & -2.11026600 & -1.51114000 \\
\hline H & -4.52904700 & 0.19496200 & 1. 96357700 \\
\hline H & -3.53932500 & 1. 66547500 & -1.92293700 \\
\hline $\mathrm{C}$ & 5. 83732100 & -0.04238600 & -2.15867800 \\
\hline $\mathrm{C}$ & 6. 80248600 & 1. 03543800 & -2.70844800 \\
\hline H & 7. 37567100 & 0.63926000 & -3.55592900 \\
\hline $\mathrm{H}$ & 7. 51256600 & 1. 35750700 & -1.93777800 \\
\hline H & 6. 26127200 & 1. 92192100 & -3.05661900 \\
\hline $\mathrm{C}$ & 6. 66931300 & -1.27249700 & -1.74856100 \\
\hline $\mathrm{H}$ & 7. 19889100 & -1.66559500 & -2.62380700 \\
\hline H & 6. 03585200 & -2.07595800 & -1.35636900 \\
\hline H & 7. 42426600 & -1.02438500 & -0.99333600 \\
\hline $\mathrm{C}$ & 4. 86703300 & -0.47846400 & -3.28181300 \\
\hline $\mathrm{H}$ & 4. 27315200 & 0.35925100 & -3.66130000 \\
\hline H & 4. 17313600 & -1.24735800 & -2.92719300 \\
\hline H & 5. 43206100 & -0.89244600 & -4.12618600 \\
\hline $\mathrm{C}$ & -4.55291100 & -0.45431400 & -3.43060600 \\
\hline $\mathrm{C}$ & -3.87929000 & -1.79594900 & -3.80310300 \\
\hline $\mathrm{H}$ & -2.83472700 & -1.81784300 & -3.47195200 \\
\hline H & -3.89340000 & -1.93949000 & -4.89064800 \\
\hline $\mathrm{H}$ & -4.39261900 & -2.65244400 & -3.35309000 \\
\hline $\mathrm{C}$ & -3.81802300 & 0.67024000 & -4.18538000 \\
\hline $\mathrm{H}$ & -2.76609300 & 0.74184000 & -3.88680700 \\
\hline H & -4.28587200 & 1. 64710200 & -4.01940900 \\
\hline $\mathrm{H}$ & -3.84804900 & 0.47127800 & -5.26277600 \\
\hline $\mathrm{C}$ & -5.73129100 & -2.15769100 & 1. 26072000 \\
\hline $\mathrm{C}$ & -4.65543500 & -2.69373300 & 2. 23436500 \\
\hline $\mathrm{H}$ & -5.09196900 & -3.43608700 & 2. 91402300 \\
\hline $\mathrm{H}$ & -4.22321400 & -1.89496100 & 2. 84554300 \\
\hline
\end{tabular}


$-6.88055800-1.51411700$

2. 07316400

$-6.52632900-0.68323600$

2. 69243900

$-7.33813300$

$-2.25492400$

2. 74055800

$-7.66157600$

$-1.12615800$

1. 40906600

$-6.31039900$

$-3.35535500$

0. 48373400

$-5.53906100$

$-3.87866200$

$-0.09285000$

$-7.10573000$

$-3.05020100$

$-0.20602600$

$-6.74296500$

$-4.07687400$

1. 18617500

4. 69008400

0. 09707100

2. 83509800

6. 16265600

0. 34455600

3. 24251400

6. 86098500

$-0.15323600$

2. 56143900

6. 35033800

$-0.03850900$

4. 25353700

6. 39582500

1. 41546800

3. 23563600

4. 40102800

$-1.42207800$

2. 87348700

5. 03871500

$-1.97864000$

2. 17876200

3. 35723000

$-1.63179800$

2. 61461300

4. 58444900

$-1.81804600$

3. 88012700

3. 78315400

0. 77904400

3. 87714800

3. 98109900

1. 85434900

3. 95024400

3. 96593900

0. 34353500

4. 86641600

2. 72131000

0. 64369400

3. 64234800

$-6.02502500$

$-0.47455500$

$-3.90740200$

$-6.52609700$

0. 47063700

$-3.66893700$

$-6.59396900$

$-1.28228800$

$-3.43451900$

$-6.07478500$

$-0.62127700$

$-4.99364300$

2. 33856600

$-5.97376900$

-3. 17148000 
INT-I (Table S4, entry 5)

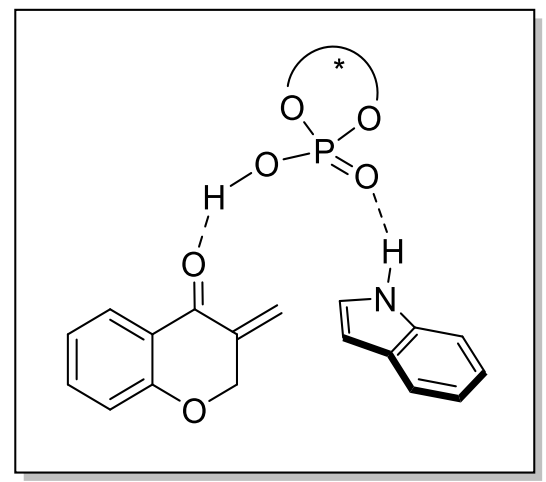

SCF energy [B3LYP-GD3BJ/6-311+G**/SMD (Chlorobenzene)]: -3291.678118080 a.u.

Thermal correction to Gibbs free energy at $258 \mathrm{~K}: 1.104907000$ a.u.

Gibbs free energy at $258 \mathrm{~K}$ [B3LYP-GD3BJ/6-311+G**/SMD (Chlorobenzene)]: -3290.573211080 a.u.

\begin{tabular}{crrr}
\hline 1 & & & \\
$\mathrm{H}$ & -0.72557700 & -0.35875500 & 0.96349900 \\
$\mathrm{C}$ & -1.28023700 & -5.41548000 & 2.15899900 \\
$\mathrm{C}$ & -2.22371800 & -3.86173700 & 3.67585200 \\
$\mathrm{C}$ & -1.60160700 & -2.77300300 & 3.02111400 \\
$\mathrm{C}$ & -1.08431700 & -2.94455200 & 1.65381300 \\
$\mathrm{C}$ & -1.10697500 & -4.33375200 & 1.12700200 \\
$\mathrm{H}$ & -3.31346300 & -4.53055600 & 5.39596700 \\
$\mathrm{H}$ & -0.33572400 & -5.57575900 & 2.70193100 \\
$\mathrm{C}$ & -2.83597300 & -3.67899800 & 4.92247700 \\
$\mathrm{C}$ & -1.58361500 & -1.51594700 & 3.65914800 \\
$\mathrm{C}$ & -2.17080700 & -1.34212000 & 4.90190700 \\
$\mathrm{C}$ & -2.80555600 & -2.42859400 & 5.52568300 \\
$\mathrm{H}$ & -1.10910300 & -0.68112100 & 3.15447500 \\
$\mathrm{H}$ & -2.14669500 & -0.37111400 & 5.38641600 \\
$\mathrm{H}$ & -3.27361100 & -2.29606900 & 6.49748600 \\
O & -0.68791600 & -2.00591000 & 0.94954200 \\
O & -2.29017900 & -5.10522500 & 3.13552900 \\
$\mathrm{H}$ & -0.95561900 & -5.57401300 & -0.58983200 \\
$\mathrm{H}$ & -0.73796200 & -3.75248300 & -0.86812300 \\
$\mathrm{H}$ & -1.58390500 & -6.36279600 & 1.70976000 \\
$\mathrm{P}$ & 0.21238800 & 1.33956400 & -0.05377300 \\
O & -0.70796800 & 2.45440600 & -0.79631500 \\
O & 1.17392600 & 2.21137700 & 0.92880300 \\
O & 0.88853400 & 0.49195600 & -1.06068700 \\
O & -0.69721800 & 0.63959000 & 1.03560600 \\
& & & \\
\hline & & & \\
& & &
\end{tabular}




\begin{tabular}{|c|c|c|c|}
\hline C & -1.53341100 & 3. 28183200 & -0.02178100 \\
\hline $\mathrm{C}$ & -2.88277200 & 2. 92282300 & 0.16980200 \\
\hline $\mathrm{C}$ & -3.64407000 & 3. 71994300 & 1. 04139600 \\
\hline C & -3.09320200 & 4. 80778600 & 1. 71777200 \\
\hline $\mathrm{C}$ & -1.76774400 & 5. 15364900 & 1. 47883900 \\
\hline C & -0.95026600 & 6. 26366000 & 2. 10300000 \\
\hline $\mathrm{C}$ & 0.49383000 & 5. 86156400 & 1. 73268400 \\
\hline C & 0.35673500 & 5. 10910900 & 0.36637100 \\
\hline C & -0.98977900 & 4. 41943900 & 0.56946400 \\
\hline C & 0.29236300 & 6. 15065100 & -0.80212600 \\
\hline C & 1. 76724700 & 6. 50483000 & -1.09221400 \\
\hline $\mathrm{C}$ & 2. 48483700 & 5. 22170900 & -0.73561900 \\
\hline $\mathrm{C}$ & 3. 77566400 & 4. 82237000 & -1.06435400 \\
\hline C & 4. 22020400 & 3. 56380100 & -0.66294500 \\
\hline $\mathrm{C}$ & 3. 38671800 & 2. 65883800 & 0.01795300 \\
\hline $\mathrm{C}$ & 2. 08024100 & 3. 09263800 & 0.32166600 \\
\hline C & 1. 64366300 & 4. 37517000 & 0.00224300 \\
\hline H & -4.68979500 & 3. 46702700 & 1. 18918600 \\
\hline H & -3.69920700 & 5. 37930700 & 2. 41654500 \\
\hline H & -1.21415300 & 7. 24172700 & 1. 67633300 \\
\hline H & -1.10356200 & 6. 34149900 & 3. 18549700 \\
\hline H & 1. 18188100 & 6. 71070200 & 1. 67294800 \\
\hline H & 0.88778900 & 5. 16431600 & 2. 48083300 \\
\hline H & -0.32389900 & 7. 02043800 & -0.55353400 \\
\hline H & -0.15108800 & 5. 66726200 & -1.67998900 \\
\hline H & 2. 10836400 & 7. 34178800 & -0.46636000 \\
\hline H & 1.93314200 & 6. 80338500 & -2.13359800 \\
\hline $\mathrm{H}$ & 4. 43443300 & 5. 47909500 & -1.62709300 \\
\hline H & 5. 23719600 & 3. 25728200 & -0.88804700 \\
\hline $\mathrm{C}$ & 3. 89862300 & 1. 30635800 & 0.37007400 \\
\hline C & 4. 67658200 & 0.59822500 & -0.55054600 \\
\hline C & 3. 65792100 & 0.73936300 & 1. 63367600 \\
\hline $\mathrm{C}$ & 5. 21928500 & -0.65956200 & -0.24770700 \\
\hline C & 4. 17227500 & -0.51213800 & 1. 97428600 \\
\hline C & 4. 94998400 & -1.19108500 & 1. 01750900 \\
\hline $\mathrm{C}$ & -3.51927100 & 1. 76277800 & -0.51286500 \\
\hline $\mathrm{C}$ & -3.35050500 & 1. 55687100 & -1.89356300 \\
\hline $\mathrm{C}$ & -4.36082300 & 0.90482000 & 0.20077900 \\
\hline C & -4.01292700 & 0.52454200 & -2.55860300 \\
\hline C & -5.04837700 & -0.14435400 & -0.42965900 \\
\hline $\mathrm{C}$ & -4.85849700 & -0.30952300 & -1.80407600 \\
\hline H & 5. 35309000 & -2.16340900 & 1. 27552700 \\
\hline H & 4. 84033100 & 1. 03240000 & -1.53141000 \\
\hline $\mathrm{H}$ & 3. 06434600 & 1. 29832800 & 2. 34471800 \\
\hline
\end{tabular}


$-2.70023100$

2. 22931500

$-2.43756900$

6. 10227300

$-1.38037200$

$-1.28601700$

7. 41546800

$-0.57936600$

$-1.45870100$

8. 06507100

$-1.06255800$

$-2.19922300$

7. 96593900

$-0.51572700$

$-0.51267000$

7. 21999900

0. 44299000

$-1.80031700$

6. 46195100

$-2.81600200$

$-0.85754500$

7. 07440800

$-3.28752900$

$-1.63447000$

5. 56497600

$-3.43137000$

$-0.72431200$

7. 04162500

$-2.83849400$

0. 07256000

5. 37351000

$-1.46488200$

$-2.64777900$

5. 14554500

$-0.47551200$

$-3.05693900$

4. 43274700

$-2.01689600$

$-2.55879500$

6. 00567900

$-1.98376500$

$-3.37879600$

$-3.86816000$

0. 28811100

$-4.07466200$

$-3.36211500$

$-1.15074000$

$-4.33343000$

$-2.38007100$

$-1.31562600$

$-3.87708800$

$-3.26700000$

$-1.33119700$

$-5.41131400$

$-4.04764900$

$-1.90483400$

$-3.93260400$

$-2.87385000$

1. 26749300

$-4.72749000$

$-1.87194400$

1. 18171300

$-4.29220000$

$-3.20108700$

2. 30887500

$-4.62838600$

$-2.79162300$

1. 04921100

$-5.79838700$

$-5.97006300$

$-1.05942500$

0. 39985500

$-5.13869800$

$-1.77954900$

1. 48727700

$-5.78390600$

$-2.42140400$

2. 10002900

$-4.63823100$

$-1.07258000$

2. 15683100

$-4.36658900$

$-2.41029500$

1. 03244400

$-7.06987700$

$-0.20748900$

1. 07721100

$-6.64619300$

0. 54688000

1. 74822200

$-7.73594000$

$-0.84565900$

1. 67127900

$-7.67727500$

0. 31442400

0. 32879300

$-6.66338100$

-2. 13425300

$-0.45919500$

$-5.93925200$

$-2.79827000$

$-0.94464600$

$-7.29582600$

$-1.69107400$

$-1.23689700$

$-7.30618300$

$-2.75498800$

0. 17546300

3. 91971500

$-1.16257100$

3. 34879400

5. 27040200

$-1.42417700$

4. 05688600

5. 91575800

$-2.08964600$

3. 47416300

5. 10558900

$-1.89190800$

5. 03552900

5. 81530300

$-0.48664800$

4. 21630500

3. 17571200

$-2.50522000$

3. 15275700 
3. $06153900-0.27551300$

4. 27114400

3. $54517300 \quad 0.68596900$

4. 47740900

2. 90963700

$-0.78142800$

5. 23164300

2. 07359900

$-0.07498400$

3. 84151100

$-5.24531400$

0. 47498400

$-4.75533800$

$-5.62639700$

1. 49049500

$-4.59678000$

$-5.99125300$

$-0.22610800$

$-4.36585300$

$-5.16240300$

0. 30750000

$-5.83639300$

1. 23085400

$-2.79275100$

$-3.02518800$

1. 63776500

$-4.15986900$

$-2.98891900$

1. 41175700

$-4.96283900$

$-4.12268000$

0. 80269700

$-4.40303400$

$-5.23811000$

0. 41106600

$-3.04673800$

$-5.25224200$

0. 62026000

$-2.22529100$

$-4.15095700$

2. 15082000

$-3.17973300$

$-1.01971700$

0. 62595800

$-5.01564500$

-6. 11854600

$-0.05922500$

$-2.63673400$

-6. 14217400

0. 33046600

$-1.17813000$

$-4.15863000$

1. 36433100

$-1.26360500$

$-1.53374400$

2. 48099300

$-2.92153500$

$-0.02363300$

2. 64740800

$-5.29779500$

$-1.31829400$

1. 55960500

$-2.22567400$

$-1.81381200$

1. 71450300

$-6.00759100$

$-4.12573800$

$-0.92689300$

$-4.56844200$

$-0.17960000$

2. 22142400

$-4.37735300$

$-1.69506100$ 
TS-I' (Table S4, entry 6)

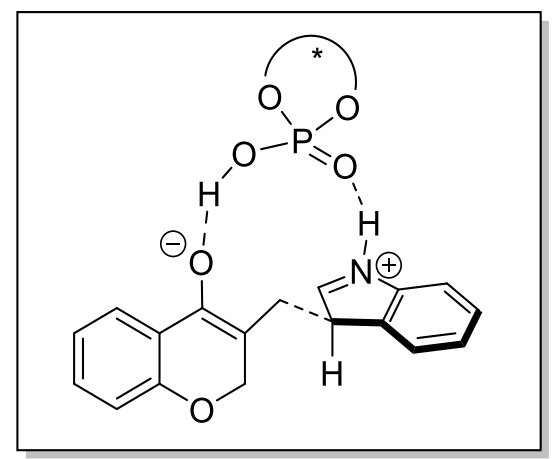

SCF energy [B3LYP-GD3BJ/6-311+G**/SMD (Chlorobenzene)]: -3291.665888740 a.u.

Thermal correction to Gibbs free energy at $258 \mathrm{~K}: 1.113042000$ a.u. Gibbs free energy at $258 \mathrm{~K}$ [B3LYP-GD3BJ/6-311+G**/SMD (Chlorobenzene)]: -3290.552846740 a.u.

$\begin{array}{lrrr}0 \mathrm{H} & & & \\ \mathrm{H} & -1.16041000 & -0.99009300 & 1.03478800 \\ \mathrm{C} & 1.63644300 & -2.96948400 & -1.80654100 \\ \mathrm{C} & 0.93918300 & -4.08780500 & -2.32325300 \\ \mathrm{C} & 1.63148900 & -5.28291700 & -2.54487800 \\ \mathrm{C} & 2.99029100 & -5.33513100 & -2.23678600 \\ \mathrm{C} & 3.65585300 & -4.22012200 & -1.69684100 \\ \mathrm{C} & 2.98949600 & -3.01507600 & -1.47255400 \\ \mathrm{C} & -0.46774100 & -2.29593400 & -2.14197700 \\ \mathrm{C} & -0.46142800 & -3.68780800 & -2.42832400 \\ \mathrm{H} & 3.54483900 & -6.25276900 & -2.41136900 \\ \mathrm{H} & 4.71210700 & -4.29575900 & -1.45452400 \\ \mathrm{H} & 3.49848700 & -2.15004700 & -1.05827700 \\ \mathrm{H} & 0.88097100 & -0.92132500 & -1.39155000 \\ \mathrm{H} & -1.29530800 & -1.60057100 & -2.14445900 \\ \mathrm{H} & -1.18919500 & -4.15172500 & -3.08308300 \\ \mathrm{~N} & 0.73167200 & -1.90642100 & -1.71738500 \\ \mathrm{C} & 0.46106400 & -5.13772100 & 0.95840600 \\ \mathrm{C} & 0.56368600 & -4.06755100 & 3.05914600 \\ \mathrm{C} & 0.07580500 & -2.85548200 & 2.50581700 \\ \mathrm{C} & -0.62545700 & -2.93326600 & 1.22172000 \\ \mathrm{C} & -0.56748300 & -4.13386700 & 0.50377300 \\ \mathrm{H} & 1.43000600 & -5.04951100 & 4.75039000 \\ \mathrm{H} & 1.47291100 & -4.87082400 & 0.61657200 \\ \mathrm{C} & 1.08062400 & -4.10039000 & 4.35721000 \\ \mathrm{C} & 0.14998100 & -1.68314400 & 3.28747500 \\ \mathrm{C} & 0.68775800 & -1.71788700 & 4.56736000\end{array}$




\begin{tabular}{|c|c|c|c|}
\hline C & 1.14428100 & -2.92906500 & 5. 10343500 \\
\hline H & -0.21164500 & -0.74532100 & 2. 88002400 \\
\hline H & 0.74323500 & -0.80518900 & 5. 15235300 \\
\hline H & 1. 55790000 & -2.95828500 & 6. 10805600 \\
\hline 0 & -1.39155500 & -1.97088800 & 0.76055400 \\
\hline 0 & 0.50050300 & -5.24851800 & 2. 39230400 \\
\hline C & -1.35411100 & -4.33617100 & -0.64556100 \\
\hline H & -1.44327200 & -5.35419900 & -1.01587400 \\
\hline $\mathrm{H}$ & -2.24647700 & -3.73133100 & -0.76155000 \\
\hline H & 0.23459800 & -6.14245700 & 0.59439300 \\
\hline P & -0.21725700 & 1. 27661000 & 0.07060800 \\
\hline 0 & -1.42608000 & 2. 21743100 & -0.55608100 \\
\hline 0 & 0.72735400 & 2. 38051800 & 0.85274600 \\
\hline 0 & 0.48600500 & 0.67179100 & -1.10942100 \\
\hline 0 & -0.76052200 & 0.41459000 & 1. 19493300 \\
\hline $\mathrm{C}$ & -2.29481600 & 2. 80349500 & 0.35933800 \\
\hline C & -3.49973600 & 2. 15413200 & 0.70023000 \\
\hline C & -4.27458100 & 2. 71515100 & 1. 72959300 \\
\hline C & -3.86854200 & 3. 85621400 & 2. 42130900 \\
\hline $\mathrm{C}$ & -2.69790800 & 4. 49917200 & 2. 03325000 \\
\hline $\mathrm{C}$ & -2.04237000 & 5. 72647500 & 2. 63034400 \\
\hline C & -0.62836500 & 5. 68830700 & 2. 00701900 \\
\hline C & -0.82847100 & 4. 99599200 & 0.61853200 \\
\hline C & -1.93441800 & 4. 00538700 & 0.96480700 \\
\hline $\mathrm{C}$ & -1.33969600 & 6. 04779500 & -0.42556000 \\
\hline $\mathrm{C}$ & -0.06329700 & 6. 74025700 & -0.94564000 \\
\hline C & 0.96643900 & 5. 63784400 & -0.83988300 \\
\hline C & 2. 21701100 & 5. 55683300 & -1.44170100 \\
\hline C & 2. 99467100 & 4. 42077400 & -1.22994800 \\
\hline $\mathrm{C}$ & 2. 53800500 & 3. 33236800 & -0.46513300 \\
\hline C & 1. 25887600 & 3. 43826500 & 0.12313600 \\
\hline C & 0.49736100 & 4. 59794000 & -0.02330500 \\
\hline H & -5.21231500 & 2. 23370100 & 1. 99187600 \\
\hline H & -4.46806600 & 4. 23837400 & 3. 24403300 \\
\hline H & -2.57885000 & 6. 64392200 & 2. 34906800 \\
\hline H & -2.02141800 & 5. 69967900 & 3. 72610600 \\
\hline H & -0.16490100 & 6. 67646600 & 1. 91937600 \\
\hline H & 0.03006200 & 5. 06241300 & 2. 61994200 \\
\hline H & -2.06942700 & 6. 74193300 & 0.00344300 \\
\hline H & -1.83001900 & 5. 51432000 & -1.24778800 \\
\hline H & 0.20894500 & 7. 60110600 & -0.31795400 \\
\hline H & -0.17300200 & 7. 11938900 & -1.96835100 \\
\hline H & 2. 58842000 & 6. 36533600 & -2.06673300 \\
\hline $\mathrm{H}$ & 3. 98703300 & 4. 36305400 & -1.66718200 \\
\hline
\end{tabular}




\begin{tabular}{|c|c|c|c|}
\hline C & 3. 42268100 & 2. 14457300 & -0.31150800 \\
\hline $\mathrm{C}$ & 4. 14933400 & 1. 67742300 & -1.41103400 \\
\hline $\mathrm{C}$ & 3. 61877800 & 1. 53071400 & 0.93803800 \\
\hline $\mathrm{C}$ & 5. 07719100 & 0.63100600 & -1.29672300 \\
\hline $\mathrm{C}$ & 4. 52543100 & 0.48145400 & 1. 09635300 \\
\hline $\mathrm{C}$ & 5.24335800 & 0.04877800 & -0.03575200 \\
\hline $\mathrm{C}$ & -3.98569200 & 0.93414900 & -0.00114700 \\
\hline C & -3.97409700 & 0.86007600 & -1.40645100 \\
\hline C & -4.55587000 & -0.11681400 & 0.72213600 \\
\hline $\mathrm{C}$ & -4.53960100 & -0.22287800 & -2.08121700 \\
\hline $\mathrm{C}$ & -5.12982800 & -1.22849600 & 0.08481800 \\
\hline $\mathrm{C}$ & -5.11033800 & -1.25629200 & -1.31261200 \\
\hline H & 5. 95960000 & -0.75603500 & 0.08407600 \\
\hline $\mathrm{H}$ & 3. 97595100 & 2. 14105500 & -2.37712500 \\
\hline H & 3. 05561500 & 1. 90120700 & 1. 78399600 \\
\hline H & -5.56644300 & -2.09263500 & -1.83004400 \\
\hline H & -4.54246200 & -0.06242900 & 1. 80636700 \\
\hline H & -3.53353100 & 1. 68039500 & -1.95762000 \\
\hline C & 5. 88935800 & 0.19764300 & -2.53406100 \\
\hline $\mathrm{C}$ & 6. 75538200 & 1. 38945500 & -3.00973500 \\
\hline H & 7. 34887100 & 1. 10606600 & -3.88806200 \\
\hline $\mathrm{H}$ & 7. 44560300 & 1. 71121400 & -2.22142400 \\
\hline H & 6. 13946300 & 2. 25145700 & -3.28656200 \\
\hline $\mathrm{C}$ & 6. 83099800 & -0.98556300 & -2.23936300 \\
\hline $\mathrm{H}$ & 7. 37958400 & -1.25590500 & -3.14886500 \\
\hline H & 6. 28016300 & -1.87475400 & -1.91096400 \\
\hline H & 7. 57057100 & -0.73824200 & -1.46949200 \\
\hline $\mathrm{C}$ & 4. 93497300 & -0.22192900 & -3.67712700 \\
\hline $\mathrm{H}$ & 4. 25757600 & 0.58876300 & -3.96299600 \\
\hline H & 4. 32109900 & -1.08123000 & -3.38498600 \\
\hline H & 5. 51033900 & -0.50491800 & -4.56751000 \\
\hline $\mathrm{C}$ & -4.61901400 & -0.28753000 & -3.62053000 \\
\hline $\mathrm{C}$ & -3.98111800 & -1.59782100 & -4.13875000 \\
\hline $\mathrm{H}$ & -2.91043900 & -1.63208000 & -3.90542400 \\
\hline $\mathrm{H}$ & -4.08573000 & -1.66691600 & -5.22854300 \\
\hline H & -4.45174000 & -2.48601400 & -3.70401700 \\
\hline $\mathrm{C}$ & -3.89328100 & 0.89114700 & -4.29740400 \\
\hline $\mathrm{H}$ & -2.83338000 & 0.93128900 & -4.02230300 \\
\hline H & -4.34791100 & 1. 85308000 & -4.03666100 \\
\hline $\mathrm{H}$ & -3.95268900 & 0.78444800 & -5.38681000 \\
\hline $\mathrm{C}$ & -5.77976200 & -2.33989400 & 0.93265400 \\
\hline C & -4.74317700 & -2.92741600 & 1.91841400 \\
\hline $\mathrm{H}$ & -5.20706200 & -3.70566400 & 2. 53718900 \\
\hline H & -4.33827200 & -2.16406100 & 2. 58986600 \\
\hline
\end{tabular}


$-3.89881800 \quad-3.37293000$

1. 38216000

$-6.96043400-1.74110200$

1. 73482900

$-6.63051800-0.94076800$

2. 40549600

$-7.43813600 \quad-2.51499600$

2. 34872600

$-7.71915200-1.32259400$

1. 06346400

$-6.32751300$

$-3.49432000$

0.07236200

$-5.53747100$

$-3.97468900$

$-0.51689700$

$-7.10978300$

$-3.15687700$

$-0.61707400$

$-6.76810800$

$-4.26108000$

0.71975600

4. 77882200

$-0.19366600$

2. 45897000

6. 26923600

$-0.03871000$

2. 84502500

6. 93448000

$-0.50337700$

2. 10944700

6. 46342700

$-0.51280000$

3. 81528800

6. 54486800

1. 01926800

2. 92277600

4. 42937500

$-1.69798400$

2. 36415200

5. 03938100

$-2.21084400$

1. 61236200

3. 37587700

$-1.83954000$

2. 09870500

4. 60258900

$-2.19407100$

3. 32717500

3. 92862300

0. 42460100

3. 58501200

4. 15431500

1. 48746500

3. 72805700

4. 14068300

$-0.08901600$

4. 52995300

2. 85631400

0. 32478400

3. 38709500

-6. 10607200

$-0.24926400$

$-4.04895800$

$-6.58651700$

0. 67316400

$-3.70386800$

$-6.66950300$

$-1.09287700$

$-3.63598900$

-6. 19218600

$-0.29021800$

$-5.14212400$

1. 12391900

$-6.15351800$

$-2.95246300$ 
TS-I (Table S4, entry 7)

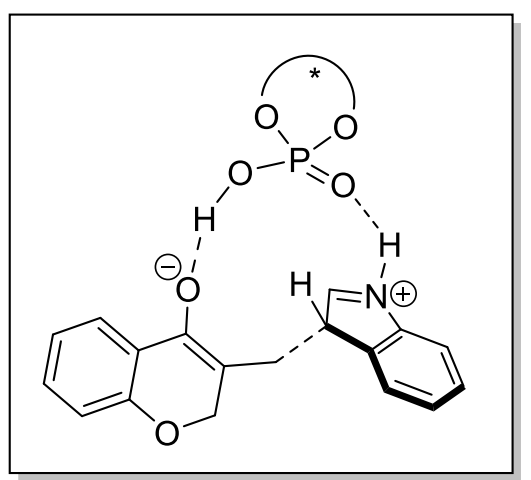

SCF energy [B3LYP-GD3BJ/6-311+G**/SMD (Chlorobenzene)]: -3291.661159890 a.u.

Thermal correction to Gibbs free energy at $258 \mathrm{~K}: 1.110038000$ a.u. Gibbs free energy at $258 \mathrm{~K}$ [B3LYP-GD3BJ/6-311+G**/SMD (Chlorobenzene)]: -3290.551121890 a.u.

\begin{tabular}{|c|c|c|c|}
\hline $\mathrm{H}$ & -0.68841100 & -1.06727400 & 0.83940300 \\
\hline $\mathrm{C}$ & 1. 16749200 & -4.93721100 & 2. 31425000 \\
\hline C & -0.02033900 & -3.68535600 & 3. 93715300 \\
\hline $\mathrm{C}$ & -0.22484200 & -2.61358400 & 3. 03264900 \\
\hline $\mathrm{C}$ & -0.04280200 & -2.89035400 & 1. 60900300 \\
\hline $\mathrm{C}$ & 0.53972800 & -4.10647000 & 1. 22575700 \\
\hline $\mathrm{H}$ & -0.14038900 & -4.37264400 & 5. 95964700 \\
\hline $\mathrm{H}$ & 2. 18605000 & -4.59029000 & 2. 56245900 \\
\hline $\mathrm{C}$ & -0.29429100 & -3.52691200 & 5. 29741100 \\
\hline $\mathrm{C}$ & -0.67840600 & -1.37737000 & 3. 53698500 \\
\hline $\mathrm{C}$ & -0.92528500 & -1.21897600 & 4. 89500700 \\
\hline $\mathrm{C}$ & -0.74195500 & -2.29717500 & 5. 77032100 \\
\hline $\mathrm{H}$ & -0.83684900 & -0.54557600 & 2. 85786200 \\
\hline $\mathrm{H}$ & -1.26737200 & -0.26017000 & 5. 27191900 \\
\hline H & -0.94189100 & -2.17564600 & 6.83159300 \\
\hline 0 & -0.41075600 & -2.07767000 & 0.65498400 \\
\hline 0 & 0.39459900 & -4.91415500 & 3. 52580400 \\
\hline $\mathrm{H}$ & 0.93679100 & -5.43927700 & -0.40939100 \\
\hline $\mathrm{H}$ & -0.04652600 & -3.95334300 & -0.82111900 \\
\hline $\mathrm{H}$ & 1. 23207400 & -5.99130000 & 2. 03233700 \\
\hline P & -0.31941200 & 1. 25521400 & -0.18257400 \\
\hline 0 & -1.51155900 & 2. 18597200 & -0.83555000 \\
\hline 0 & 0.53478500 & 2. 35090700 & 0.71331900 \\
\hline 0 & 0.47702800 & 0.73501900 & -1.33929700 \\
\hline 0 & -0.89707700 & 0.32667700 & 0.87419500 \\
\hline $\mathrm{C}$ & -2.41076300 & 2. 79156100 & 0.03604900 \\
\hline
\end{tabular}


$-4.08467900$

3. 93651400

1. 96293400

$-2.90800200$

4. 57544200

1. 58601300

$-2.29975700$

5. 84798300

2. 13497200

$-0.84804200$

5. 77381800

1. 61275600

$-0.95142300$

4. 99321200

0. 25884200

$-2.08623400$

4. 02866000

0. 58861100

$-1.35386400$

5. 98098100

$-0.88798900$

$-0.02635600$

6. 63915400

$-1.32331200$

0. 98387700

5. 54676100

$-1.04759600$

2. 29233500

5. 43441500

$-1.50538600$

3. 03950000

4. 31214900

$-1.15054300$

2. 49251400

3. 26297200

-0. 39177600

1. 15407500

3. 39414100

0. 03640800

0. 42318700

4. 54981000

$-0.23533800$

$-5.36913600$

2. 24850500

1. 60141700

$-4.72633100$

4. 36055500

2. 73169300

$-2.81766100$

6. 73769800

1. 74890300

$-2.35355700$

5. 90369400

3. 22852300

$-0.37649100$

6. 75491700

1. 49510200

$-0.23489800$

5. 18973400

2. 30847400

$-2.11473700$

6. 70172500

$-0.57128900$

$-1.76868000$

5. 40098900

$-1.72017900$

0. 18708500

7. 54012000

$-0.73022800$

$-0.03302000$

6. 95008900

$-2.37451100$

2. 73313600

6. 20955800

$-2.12761500$

4. 07472800

4. 23474200

$-1.47010900$

3. 33516900

2. 07984600

$-0.06432500$

4. 18462400

1. 53443900

$-1.03161500$

3. 35681300

1. 53944300

1. 23464900

5. 06072700

0. 47735500

$-0.73814500$

4. 21218000

0. 48870700

1. 57031600

5. 05493800

$-0.02630800$

0. 56602300

$-4.01055100$

0. 83725300

$-0.23447200$

$-3.95752900$

0.64914600

$-1.62641800$

$-4.48795000$

$-0.20048600$

0. 56934700

$-4.37282400$

$-0.54690100$

$-2.21186200$

$-4.91450100$

$-1.42048200$

0. 02173200

$-4.84993400$

$-1.56566300$

$-1.36651800$

5. 73030200

$-0.83475800$

0. 82215800

4. 14755000

1. 94046900

$-2.03773900$

2. 69909000

1. 96905500

1. 97838500

$-5.17270800$

$-2.49840900$

$-1.81456600$ 
$-4.49631200$

$-3.58377600$

$-0.05576600$

1. 45942800

5. 99714900

6. 94188000

7. 61913000

7. 55131800

6. 38412600

6. 86641800

7. 50442100

6. 25580300

7. 52369200

5. 16381800

4. 53872700

4. 50282200

5. 82434500

$-4.32496100$

$-3.41685100$

$-2.39478900$

$-3.37666800$

$-3.78242600$

$-3.76621700$

$-2.74554300$

$-4.38846800$

$-3.74200400$

$-5.40191200$

$-4.24341000$

$-4.56288000$

$-3.89866400$

$-3.38526000$

$-6.59152400$

$-6.31582300$

$-6.94397100$

$-7.42992500$

$-5.86796200$

$-5.05657500$

$-6.69902100$

$-6.21483300$

4. 29250900

5. 71515800

6. 48141900

5. 79496400

5. 94846400

3. 99928300

4. 69737300
$-0.06276500$

1. 07210900

0. 71200000

1. 43898100

1. 92266300

$-1.23699900$

$-1.58940400$

$-2.08503900$

$-0.94449400$

-0. 55591600

0. 23940200

$-1.38030900$

$-0.91592900$

$-0.78410900$

$-1.99928700$

$-1.82341700$

$-2.18383500$

$-2.91355800$

0. 43050300

0. 67573300

1. 32169900

0. 21034700

$-2.54458900$

$-2.96269500$

$-3.76973800$

$-2.12862200$

$-3.31983600$

$-2.03873800$

$-1.17693200$

$-2.82981600$

$-1.73820900$

$-3.79388700$

$-4.23672700$

$-3.56801700$

$-4.55610300$

$-0.08938400$

0. 14149900

$-0.34362600$

$-0.26281300$

1. 21149300

$-1.60842500$

-2. 14894000
1. 64551800

$-2.23849900$

$-1.83756600$

$-2.30107000$

$-3.08563500$

$-1.46720000$

$-2.70640300$

$-1.34867200$

$-2.16713000$

$-1.01738100$

$-0.52181000$

$-3.04351600$

$-3.46188200$

$-2.75409800$

$-3.84202800$

$-3.73392300$

$-4.03900400$

$-3.68460600$

$-5.12011900$

$-3.55974800$

$-4.49970100$

$-4.18595800$

-4. 35996700

$-5.57346400$

0. 95602200

1. 89241100

2. 56398300

2. 51192700

1. 31184400

1. 80583300

2. 42230900

2. 47953700

1. 16679000

0. 18452100

$-0.40430200$

$-0.49358300$

0. 89170000

2. 99754000

3. 56151800

2. 94750900

4. 57842100

3. 60205200

2. 96858400

2. 32023200 
$-6.41675100$

$-0.21960200$

$-4.06481400$

$-6.19336600$

$-1.95277600$

$-3.78693100$

$-5.73292900$

$-1.24437600$

$-5.34284900$

1. 45866100

$-2.58222500$

$-3.02202700$

2. 00721100

$-3.79860800$

$-2.54515000$

2. 07709500

$-4.89779500$

$-3.40769900$

1. 60376500

$-4.75720800$

$-4.71170300$

1. 05360000

$-3.54354500$

$-5.15969800$

0. 97058400

$-2.43451000$

-4. 31928300

2. 06623400

$-2.21084500$

$-0.90599400$

1. 66034700

$-5.59963500$

$-5.39528300$

0.68898000

$-3.46678600$

-6. 17989500

0. 55005700

$-1.49311700$

$-4.65925700$

1. 10404900

$-0.69409100$

$-1.93483200$

2. 21104700

$-1.62919700$

$-0.00753700$

3. 06897600

-4. 14529100

$-0.58038500$

1. 52697300

$-1.64771900$

$-1.98588000$

2. 49690100

$-5.84296700$

$-3.07288400$

0. 65072600

$-4.42579900$

$-0.13930000$

2. 31852900

$-3.59207900$

$-1.13166500$ 
INT-II' (Table S4, entry 8)

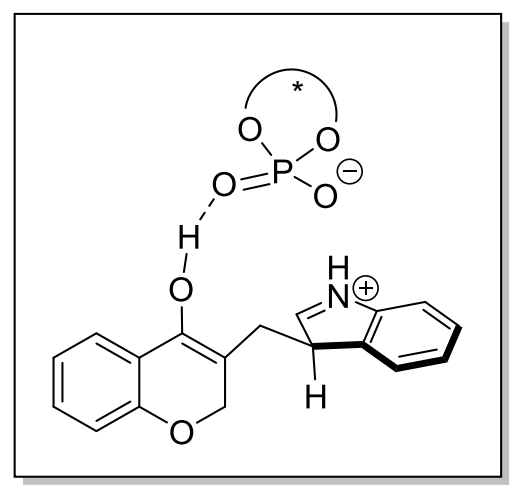

SCF energy [B3LYP-GD3BJ/6-311+G**/SMD (Chlorobenzene)]: -3291.684036610 a.u.

Thermal correction to Gibbs free energy at $258 \mathrm{~K}: 1.113856000$ a.u.

Gibbs free energy at $258 \mathrm{~K}$ [B3LYP-GD3BJ/6-311+G**/SMD (Chlorobenzene)]: -3290.570180610 a.u.

\begin{tabular}{crrr} 
O 1 & & & \\
$\mathrm{H}$ & -1.40939200 & -1.17471800 & 0.95751600 \\
$\mathrm{C}$ & 1.04338500 & -2.74836700 & -1.97249700 \\
$\mathrm{C}$ & 0.34334400 & -3.95360900 & -2.11046500 \\
$\mathrm{C}$ & 1.05033300 & -5.12933200 & -2.32729800 \\
$\mathrm{C}$ & 2.44895000 & -5.06307600 & -2.39510000 \\
$\mathrm{C}$ & 3.12415900 & -3.84738000 & -2.23773800 \\
$\mathrm{C}$ & 2.42668700 & -2.65415000 & -2.02018800 \\
$\mathrm{C}$ & -1.10581800 & -2.16490800 & -1.76007600 \\
$\mathrm{C}$ & -1.13021200 & -3.65623800 & -1.93833600 \\
$\mathrm{H}$ & 3.01803200 & -5.97219400 & -2.56635200 \\
$\mathrm{H}$ & 4.20886700 & -3.82729900 & -2.28254500 \\
$\mathrm{H}$ & 2.93751300 & -1.70706900 & -1.88016800 \\
$\mathrm{H}$ & 0.35231800 & -0.63976000 & -1.59909200 \\
$\mathrm{H}$ & -1.96327300 & -1.51362400 & -1.65458100 \\
$\mathrm{H}$ & -1.70632200 & -3.88255900 & -2.84821600 \\
$\mathrm{~N}$ & 0.10406500 & -1.69929900 & -1.78817500 \\
$\mathrm{C}$ & -0.43132900 & -5.46861800 & 1.17452100 \\
$\mathrm{C}$ & -0.23572100 & -4.29546900 & 3.23023400 \\
$\mathrm{C}$ & -0.54462600 & -3.06552700 & 2.60794000 \\
$\mathrm{C}$ & -1.16225800 & -3.13039200 & 1.27410900 \\
$\mathrm{C}$ & -1.17344200 & -4.29496500 & 0.58661600 \\
$\mathrm{H}$ & 0.46311700 & -5.30046500 & 4.98381900 \\
$\mathrm{H}$ & 0.62672800 & -5.48486400 & 0.85549700 \\
$\mathrm{C}$ & 0.25203000 & -4.33448400 & 4.53593000 \\
$\mathrm{C}$ & -0.33389200 & -1.87855700 & 3.32655500 \\
& & & \\
\hline & & & \\
& & &
\end{tabular}




\begin{tabular}{|c|c|c|c|}
\hline $\mathrm{C}$ & 0.16993900 & -1.91660800 & 4. 62621200 \\
\hline $\mathrm{C}$ & 0.45575400 & -3.14302700 & 5.23097500 \\
\hline & -0.55768100 & -0.92176700 & 2. 86620100 \\
\hline & 0.33130400 & -0.98850400 & 5. 16646200 \\
\hline & 0.84360700 & -3.17518400 & 6.24578200 \\
\hline & -1.81912100 & -2.06471300 & 0.74629800 \\
\hline & -0.46758800 & -5.49005800 & 2.60756800 \\
\hline & -1.84164700 & -4.41430800 & -0.75524100 \\
\hline & -1.90216900 & -5.46717200 & -1.05383800 \\
\hline & -2.87030500 & -4.03513600 & -0.71685900 \\
\hline & -0.87977500 & -6.41876200 & 0.86587100 \\
\hline & -0.04340100 & 1. 26443900 & 0.01837700 \\
\hline & -1.08888900 & 2. 44079300 & $-0.4870200 c$ \\
\hline & 1. 03771100 & 2. 10826900 & 0.92995600 \\
\hline & 0.59810500 & 0.75708900 & -1.25808100 \\
\hline & -0.70499000 & 0.30939500 & 0.97187800 \\
\hline & -1.90210300 & 3. 01246500 & 0.48623500 \\
\hline & -3.19468700 & 2. 49629000 & 0.71968600 \\
\hline & -3.92121300 & 3. 03113600 & 1. 79772700 \\
\hline & -3.38594600 & 4. 00604800 & 2.63924700 \\
\hline & -2.12299700 & 4. 51863200 & 2. 36179700 \\
\hline & -1.32159500 & 5. 54942800 & 3. 12829500 \\
\hline & 0.09285300 & 5. 39078100 & 2.52430400 \\
\hline & -0.15751100 & 4. 92429300 & 1. 05204700 \\
\hline & -1.40017500 & 4. 06341100 & 1.24938200 \\
\hline & -0.49146300 & 6. 16626300 & 0.15590100 \\
\hline & 0.88105900 & 6.74476400 & -0.24388600 \\
\hline & 1. 75362500 & 5. 51006200 & -0.27462500 \\
\hline & 3. 00680900 & 5. 34917800 & -0.85423300 \\
\hline & 3. 62588200 & 4. 10356600 & -0.78744600 \\
\hline & 3. 00405600 & 2. 98771600 & -0.19834400 \\
\hline & 1. 72426600 & 3. 17910500 & 0.36819500 \\
\hline & 1. 12349200 & 4. 43869200 & 0.37678100 \\
\hline & -4.92571100 & 2. 66066300 & 1. 98022400 \\
\hline & -3.95680700 & 4. 36222200 & 3. 49336500 \\
\hline & -1.71381100 & 6. 56366300 & 2. 96746700 \\
\hline & -1.34027300 & 5. 37445600 & 4. 21032500 \\
\hline & 0.69359600 & 6.30452700 & 2.57820100 \\
\hline & 0.63590400 & 4. 60051800 & 3. 05482300 \\
\hline & -1.13801700 & 6. 88760300 & 0.66561400 \\
\hline & -1.01986500 & 5. 81882300 & -0.73919300 \\
\hline & 1. 24189700 & 7. 46946600 & 0.50017600 \\
\hline & 0.85700600 & 7. 26840300 & -1.20665000 \\
\hline & 3. 50333100 & 6. 18102600 & -1.3479600 \\
\hline
\end{tabular}


3. 72897500

1. 68660900

-0. 18755400

C

4. 48640300

1. 30664800

$-1.30089600$

3. 75175300

0. 86618600

0. 95441200

C

5. 28180100

0. 15007600

$-1.29813700$

C

4. 51365100

$-0.30247500$

0. 99454600

5. 27222500

$-0.63887800$

-0. 14312400

$-3.80252200$

1. 43556900

$-0.12954100$

$-3.66888300$

1. 46427500

$-1.53063200$

$-4.58264400$

0. 42922400

0. 44826700

$-4.29930400$

0. 51869900

$-2.34105000$

$-5.23728000$

$-0.53997700$

$-0.32804900$

$-5.07676000$

$-0.47626100$

$-1.71580900$

5. 87751900

$-1.53779900$

$-0.11129300$

4. 44890000

1. 93180000

$-2.18761500$

3. 16910600

1. 16793500

1. 81388600

$-5.58065500$

$-1.20649300$

$-2.33905000$

$-4.66618000$

0. 39717200

1. 52985200

$-3.07075500$

2. 24989900

$-1.97161100$

6. 15687900

$-0.17537700$

$-2.52634500$

7. 18471300

0. 96494300

$-2.72699600$

7. 82761800

0. 75460100

$-3.59083100$

7. 82454900

1. 07373500

$-1.84396800$

6. 69204500

1. 92674100

$-2.90283500$

6. 93877500

$-1.49248300$

$-2.36162100$

7. 53563000

$-1.68383300$

$-3.26068700$

6. 27050600

$-2.35032100$

$-2.22008200$

7. 62694600

$-1.45573600$

$-1.50983100$

5. 28037100

$-0.29166900$

$-3.79534300$

4. 70790300

0. 62212400

$-3.98332000$

4. 56608400

$-1.11891600$

$-3.71092500$

5. 90783000

$-0.47866500$

$-4.67570900$

$-4.21418400$

0. 56445800

$-3.88093600$

$-3.66831900$

$-0.77612600$

$-4.42660300$

$-2.64145200$

$-0.94901700$

$-4.08164000$

$-3.65167700$

$-0.76310200$

$-5.52324700$

$-4.28096500$

$-1.62880700$

$-4.11440200$

$-3.29035300$

1. 69006900

$-4.38451700$

$-2.26916200$

1. 58339300

$-4.00212700$

$-3.65994200$

2. 67919100

$-4.09291200$

$-3.24259100$

1. 66622100

$-5.47943200$

-6. 12542800

$-1.59518300$

0. 36080800

$-5.32579500$

$-2.33578700$

1. 45762700

$-5.95720100$

$-3.09371600$

1. 93742400 
$-4.44355600-2.83077700$

1. 04023400

$-7.33579300-0.88160300$

1. 01080000

$-7.01519900-0.14327100$

1. 75351300

$-7.98248700$

$-1.60818300$

1. 51851900

$-7.93589000$

$-0.35869000$

0. 25714200

$-6.66448500$

$-2.64644000$

$-0.62815400$

$-5.85257300$

$-3.18680200$

$-1.12974900$

$-7.30306400$

$-2.19867000$

$-1.39816400$

$-7.26966500$

$-3.38427600$

$-0.08962300$

4. 56206400

$-1.21473400$

2. 23622800

6. 02472100

$-1.36031200$

2. 71940700

6. 66982200

$-1.80599700$

1. 95480100

6. $06941800-2.00359200$

3. 60688600

6. 44776600

$-0.38480200$

2. 98580400

4. 00189600

$-2.61022900$

1. 87104800

4. 56916800

$-3.07711900$

1. 05807100

2. 95504600

$-2.54133900$

1. 55517400

4. 04689900

$-3.27964400$

2. 73893700

3. 72902000

$-0.65720800$

3. 40583000

4. 09281900

0. 32426300

3. 73139400

3. 79655900

$-1.33789300$

4. 26207600

2. 66950900

$-0.56439700$

3. 14649800

$-5.62906500$

0. 80547800

$-4.46020000$

$-6.04401300$

1. 75082800

$-4.09295900$

-6. 32432400

0. 00615900

$-4.18241800$

$-5.59258700$

0. 85284900

$-5.55580800$

0. 53956600

$-6.08169300$

$-2.44196300$ 
INT-II (Table S4, entry 9)

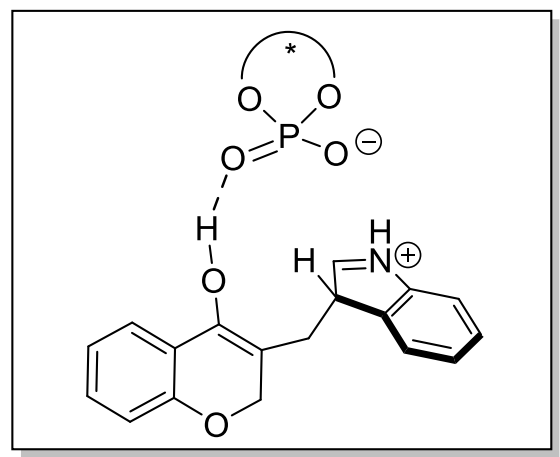

SCF energy [B3LYP-GD3BJ/6-311+G**/SMD (Chlorobenzene)]: -3291.681271260 a.u.

Thermal correction to Gibbs free energy at $258 \mathrm{~K}: 1.113184000$ a.u.

Gibbs free energy at $258 \mathrm{~K}$ [B3LYP-GD3BJ/6-311+G**/SMD (Chlorobenzene)]: -3290.568087260 a.u.

\begin{tabular}{lrrr}
01 & & & \\
$\mathrm{H}$ & -0.99314000 & -1.45115800 & 0.68623900 \\
$\mathrm{C}$ & 0.96578100 & -5.03217700 & 2.39149000 \\
$\mathrm{C}$ & -0.40145600 & -3.81987000 & 3.91529900 \\
$\mathrm{C}$ & -0.66503200 & -2.81379800 & 2.96013800 \\
$\mathrm{C}$ & -0.36877000 & -3.14198500 & 1.55910300 \\
$\mathrm{C}$ & 0.39770700 & -4.21736000 & 1.25824800 \\
$\mathrm{H}$ & -0.52940400 & -4.43560800 & 5.95971000 \\
$\mathrm{H}$ & 1.96916100 & -4.67995100 & 2.69638300 \\
$\mathrm{C}$ & -0.73020700 & -3.63312900 & 5.25688000 \\
$\mathrm{C}$ & -1.23375500 & -1.60555500 & 3.39073400 \\
$\mathrm{C}$ & -1.54284400 & -1.41090200 & 4.73670200 \\
$\mathrm{C}$ & -1.30068700 & -2.42709100 & 5.66419100 \\
$\mathrm{H}$ & -1.42029700 & -0.81152500 & 2.67453700 \\
$\mathrm{H}$ & -1.97487300 & -0.46767400 & 5.05759400 \\
$\mathrm{H}$ & -1.54645600 & -2.27964800 & 6.71253400 \\
$\mathrm{O}$ & -0.89121400 & -2.43481700 & 0.52571500 \\
$\mathrm{O}$ & 0.12085200 & -5.03107600 & 3.55062200 \\
$\mathrm{H}$ & 1.07655900 & -5.56381100 & -0.28327600 \\
$\mathrm{H}$ & -0.21639600 & -4.46446900 & -0.77590500 \\
$\mathrm{H}$ & 1.06352100 & -6.08672300 & 2.11249500 \\
$\mathrm{P}$ & -0.21102400 & 1.25981500 & -0.20076400 \\
$\mathrm{O}$ & -1.37106600 & 2.29016200 & -0.74923500 \\
$\mathrm{O}$ & 0.65594100 & 2.21848300 & 0.82937900 \\
$\mathrm{O}$ & 0.60005100 & 0.93057200 & -1.43697300 \\
$\mathrm{O}$ & -0.76336300 & 0.17328600 & 0.68056500 \\
$\mathrm{C}$ & -2.26514200 & 2.82224400 & 0.17425900 \\
& & & \\
\hline
\end{tabular}


$-3.49404300$

$-4.31937200$

$-3.94114800$

$-2.73674200$

$-2.10256600$

$-0.64405200$

$-0.74388800$

$-1.91216400$

$-1.09462400$

0. 25951000

1. 23407400

2. 54822500

3. 25482000

2. 65998300

1. 31791800

0. 62749800

$-5.27517600$

$-4.58538700$

$-2.58669100$

$-2.17585300$

$-0.14238500$

$-0.06133800$

$-1.84420800$

$-1.50339000$

0. 48239800

0. 28482900

3. 02446300

4. 29362400

3. 45131400

4. 29522500

3. 42436700

5. 11978200

4. 22846600

5. 06776500

$-3.93104100$

$-3.81113200$

$-4.51519300$

$-4.26092400$

$-4.98008300$

$-4.84400700$

5. 70501800

4. 29561000

2. 77046800

-5. 19344800
2. 17341500

0. 41753500

2. 70030400

1. 42526000

3. 80708400

2. 18483600

4. 44253400

1. 90109200

5. 62787100

5. 56617400

2. 59605100

4. 94112700

2. 09198900

3. 98037200

6. 05899900

0. 65923100

0. 86427600

6. 72430300

5. 57892700

$-0.37958700$

$-0.71181100$

$-0.54214700$

5. 47771500

$-0.98648700$

4. 29896300

3. 18898500

$-0.74936000$

3. 31395300

$-0.12604200$

0. 29120900

4. 51490500

0. 14222300

2. 21976200

1. 61376500

4. 16722900

2. 98339600

6. 57003000

5. 56556000

2. 30124000

3. 68806500

6. 53934500

2. 09037000

4. 89098500

2. 72895300

6. 76210400

-0. 00225700

5. 58503800

$-1.27914900$

7. 54540400

7. 15232600

$-0.01548900$

$-1.72067500$

6. 30388600

$-1.50892400$

4. 22502400

$-1.05831100$

1. 94412800

0. 07631800

1. 47604700

1. 25730800

$-0.93520700$

0. 35448300

1. 30431800

0. 13886800

$-0.75215500$

$-0.29442300$

0. 97549200

0. 48496800

0. 93403100

$-0.34981600$

$-1.75011000$

$-0.10931800$

0. 30907600

-0. 16330400

$-2.48376900$

$-1.23397800$

$-0.39087200$

$-1.23410500$

$-1.78144800$

$-1.15402600$

0. 65717200

1. 99692800

$-1.88776400$

1. 62631300

2. 08292900

$-2.09139600$

$-2.34483000$ 
$-4.58188800$

$-3.35838000$

6. 05208600

7. 05273000

7. 72523000

7. 66522100

6. 54022900

6. 86271000

7. 50126400

6. 21273000

7. 51499200

5. 21904000

4. 64318000

4. 51280900

5. 87646000

$-4.13925800$

-3. 28106500

$-2.27157600$

$-3.19299200$

$-3.71860500$

$-3.47350400$

$-2.46022300$

$-4.05355400$

$-3.40233600$

$-5.59247900$

$-4.53845600$

$-4.94207100$

$-4.23810500$

$-3.63385900$

$-6.82606800$

$-6.56392000$

$-7.26199600$

$-7.59860800$

$-6.04554800$

$-5.20777300$

$-6.81231800$

$-6.47650400$

4. 26160500

5. 67353700

6. 44704600

5. 72110400

5. 92226900

3. 94979400

4. 65253600
$-0.08165000$

1. 77964000

$-0.09818700$

1. 03801300

0. 73877500

1. 27877100

1. 95438100

$-1.35740800$

$-1.64022800$

$-2.21181300$

$-1.18998800$

-0. 41312700

0. 45474100

$-1.23030000$

$-0.71744200$

$-0.23818700$

$-1.46569300$

$-1.38523000$

$-1.53933400$

$-2.40183000$

1. 01603800

1. 16471100

1. 92244200

0. 91210600

$-2.41475800$

$-2.97915800$

$-3.84675800$

$-2.23730100$

$-3.29072600$

$-1.92704000$

$-1.13290300$

$-2.75374300$

$-1.53522100$

$-3.55953300$

$-3.98001500$

$-3.23210200$

$-4.36898900$

$-0.59555200$

$-0.45208300$

$-0.88212100$

$-0.96502900$

0. 60287400

$-2.09755800$

$-2.57641000$
1. 39264400

$-2.25000800$

$-1.89386400$

$-2.21462200$

$-3.02831900$

$-1.33822300$

$-2.52495000$

$-1.53273500$

$-2.37732500$

$-1.30989800$

$-0.66829200$

$-3.15875800$

$-3.49562200$

$-2.97173000$

$-3.98267900$

$-4.01850500$

$-4.40793200$

$-3.98851000$

$-5.49943300$

$-4.04537800$

$-4.61645800$

$-4.22728500$

$-4.40939000$

$-5.70567500$

0. 38730200

1. 36921500

1. 90631900

2. 11593000

0. 83627100

1. 18321500

1. 88991400

1. 75816100

0. 51118000

$-0.53870500$

$-1.10656100$

$-1.25050300$

0. 06162200

2. 88585500

3. 50409500

2. 85857000

4. 47269700

3. 66535000

2. 68633200

1. 99558900 
3. $28838900-0.59609700$

4. 82559500

2. 21042600

$-0.10484600$

3. 51238100

$-5.54652200$

$-0.38102900$

$-4.64508700$

$-6.17471200$

0. 48162300

$-4.39503000$

$-6.06046400$

$-1.28121400$

$-4.29203900$

$-5.47489400$

$-0.44409100$

$-5.73829600$

1. 51201000

$-2.36618100$

$-2.85951600$

1. 79002200

$-3.65024800$

$-2.36586100$

1. 98845600

$-4.69371900$

-3. 26089100

1. 90716100

$-4.42205600$

$-4.63328300$

1. 62822000

-3. 13298400

$-5.10129300$

1. 42351300

$-2.07207100$

$-4.21308900$

1. 47613600

$-2.13118600$

$-0.63966400$

2. 06243100

$-5.22651700$

$-5.34624300$

1. 56874100

$-2.95139000$

-6. 17017600

1. 20472900

$-1.06773100$

$-4.56096300$

1. 06441700

$-0.42491400$

$-1.74261600$

1. 41743900

$-1.60763600$

0. 30284400

2. 73936500

$-3.81285700$

$-0.40697300$

1. 35429800

$-1.48700900$

$-1.75699900$

2. 20184100

$-5.70050400$

$-2.91249300$

0. 69644500

$-4.54145100$

$-0.17826800$

1. 75983500

$-3.58737800$

$-0.85284900$ 
TS-II' (Table S4, entry 10)

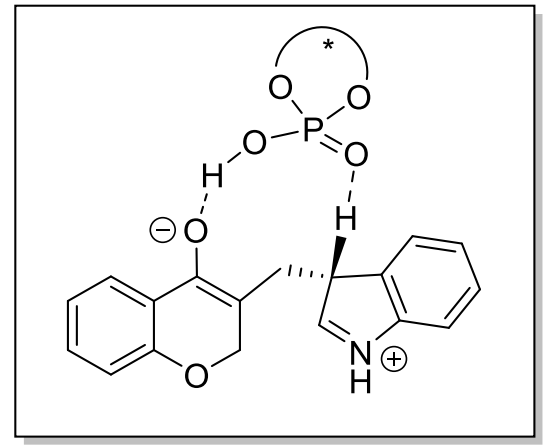

SCF energy [B3LYP-GD3BJ/6-311+G**/SMD (Chlorobenzene)]: -3291.661442480 a.u.

Thermal correction to Gibbs free energy at $258 \mathrm{~K}: 1.113140000$ a.u. Gibbs free energy at $258 \mathrm{~K}$ [B3LYP-GD3BJ/6-311+G**/SMD (Chlorobenzene)]: -3290.548302480 a.u.

01

P

0

0

0

0

C

C

C

C

C

C

C

C

C

C

C

C

C

C

C

C

C

$\mathrm{H}$

$\mathrm{H}$

$\mathrm{H}$

H
$-0.37487000$

$-1.61475700$

0. 27441400

0. 52263500

$-0.90470700$

$-2.63746400$

$-3.84689100$

$-4.81514700$

$-4.59549000$

$-3.41243700$

$-2.94898800$

$-1.45138700$

$-1.36253600$

$-2.45075800$

$-1.73526500$

-0. 42919400

0. 63542800

1. 98513900

2. 78411900

2. 25475500

0. 87603500

0. 08207400

$-5.76344800$

-5. 34937600

$-3.48921800$

$-3.11475400$
1. 27582700

1. 76834000

2. 69217600

0. 49362100

0. 61973900

2. 54728600

1. 94023500

2. 76439000

4. 12242700

4. 69821400

6. 13285800

6. 03425600

4. 83382600

3. 92509300

5. 32082300

5. 92458700

5. 10728000

4. 97660700

4. 13850700

3. 37865400

3. 50519500

4. 40223000

2. 32561100

4. 72121200

6. 78888000

6. 53888700
$-0.00777600$

$-0.98695600$

0. 51108900

$-0.91804100$

1. 26137000

$-0.46812100$

$-0.06153400$

0. 53961700

0. 76135800

0.31029000

0. 43753700

0. 07313000

$-0.92808700$

$-0.35732600$

$-2.37035000$

$-2.93293200$

$-2.23375200$

$-2.54475800$

$-1.76626400$

$-0.70979800$

$-0.44693200$

$-1.15493600$

0. 83492500

1. 26643200

$-0.25975800$

1. 44216600 
-0. 34729900

$-0.86790400$

5. 78854200

0. 96758400

$-2.57512900$

6. 02327600

$-2.37051400$

$-2.02719100$

4. 45071400

$-2.96939800$

$-0.34284100$

6. 99165400

$-2.68234800$

$-0.36755100$

5. 85267500

$-4.02497900$

2. 41865300

5. 52212400

-3. 37944300

3. 84649000

4. 05797300

$-1.97777200$

3. 14430800

2. 49893700

0. 09740200

4. 08135900

1. 66886600

$-0.53284500$

3. 10293400

2. 54070800

1. 49909100

4. 98516400

0. 89442400

0. 20625800

3. 98508400

1. 78225300

2. 27438700

4. 91751200

0.97569900

1. 60427400

-4. 15383400

0. 49902500

$-0.28627400$

$-3.78532100$

$-0.15306000$

$-1.47340900$

$-4.92485200$

$-0.21026400$

0. 64803700

$-4.18101100$

$-1.46940900$

$-1.74716800$

-5. 35178800

$-1.52313800$

0. 41769800

$-4.95747200$

$-2.13997200$

$-0.78434700$

5. 61678400

0. 38976900

2. 19315400

4. 07897500

1. 62223000

$-1.61526800$

2. 37331200

3. 18502800

1. 97193000

$-5.30406100$

$-3.14317400$

$-0.99660400$

$-5.19822900$

0. 28860100

1. 57099300

$-3.20239400$

0. 39573600

$-2.20294000$

6. 05416600

0. 00787600

$-0.46388600$

7. 44535200

0. 64117500

$-0.21957800$

8. 23147900

0. 03052600

$-0.68144900$

7. 66739400

0. 72322600

0. 85033300

7. 49968400

1. 64804500

$-0.64971200$

6. 03723000

$-1.41641100$

0. 13944600

6. 79419500

$-2.03988800$

$-0.35162000$

5. 06332300

$-1.89813800$

0. 00251500

6. 26430500

$-1.41316300$

1. 21092400

5. 83359800

$-0.11949800$

$-1.98407200$

5. 93377700

0. 84563200

$-2.49415800$

4. 84505700

$-0.53021900$

$-2.21549900$

6. 58682800

$-0.79228800$

$-2.40990300$

$-3.81937700$

-2. 10077600

$-3.10928600$

$-4.36010000$

$-3.53569200$

$-3.26103000$

$-3.95625600$

$-4.21215000$

$-2.49808700$

$-4.06842800$

$-3.93615200$

$-4.23812500$

$-5.45393900$

$-3.57064000$

$-3.20401400$ 
$-2.28356500$

-2. 14717300

$-1.82386800$

$-2.83955400$

$-1.81831600$

$-1.16455300$

$-2.02790300$

$-2.50726800$

$-6.31172700$

$-2.20702800$

$-7.71589300$

$-1.57675900$

$-8.43420900$

$-2.03950300$

$-8.08464000$

$-1.71537100$

$-7.69465800$

$-0.50116300$

$-6.43412800$

$-3.72473800$

$-6.87875100$

$-3.95322000$

$-7.08062400$

$-4.17205900$

$-5.46165900$

$-4.23043500$

$-5.84968000$

$-1.99312200$

$-5.84437700$

$-0.93650800$

$-4.84036100$

$-2.38409000$

$-6.53126700$

$-2.50894900$

3. 96322100

1. 81037600

5. 34183300

2. 26493000

6. 14649900

1. 59300400

5. 33811100

2. 28733800

5. 58631500

3. 27089000

3. 65159500

0. 39173400

4. 39446000

$-0.34090300$

2. 66672900

0. 05592100

3. 64476800

0. 38709000

2. 89529100

2. 77224000

3. 07788100

3. 80642300

2. 91435900

2. 75115200

1. 88612600

2. 49180200

$-4.43339800$

$-1.23914300$

$-4.03606300$

$-0.21944000$

$-5.52252400$

$-1.17523500$

$-4.20891400$

$-1.68152100$

-0. 34177300

$-0.72796200$

1. 65944900

$-0.70658200$

2. 43672200

$-4.14860100$

3. 55586300

$-4.70757700$

2. 99359100

$-3.59294100$

2. 40668000

$-2.52443600$

2. 18254600

$-2.75189500$

4. 63840000

$-6.54444700$

$-4.79742800$

1. 54525900

$-5.70717300$
$-3.28892400$

$-2.57227200$

$-3.15969900$

$-4.29246300$

1. 41425900

1. 23943400

1. 92767000

0. 21689100

1. 44471500

1. 16873500

0. 19413800

1. 93177600

1. 22113300

2. 87408400

3. 15740000

3. 04049000

3. 56089900

3. 81560200

4. 35003200

4. 03385800

5. 44701500

3. 99036900

4. 34998200

4. 01694600

4. 00333500

5. 44736200

4. 37080200

4. 05739100

5. 46669300

4. 04970300

$-4.23994600$

$-4.23622000$

$-4.13658500$

$-5.21807600$

1. 41383600

$-1.45492100$

0. 57244700

$-1.43625200$

$-2.08807900$

$-1.26417400$

0. 05430500

$-1.62196300$

0. 48016400

$-2.16037000$ 


$\begin{array}{lrrr}\mathrm{C} & 3.08027100 & -3.51633900 & -3.48449000 \\ \mathrm{C} & 3.71217700 & -4.52126100 & -4.21521600 \\ \mathrm{C} & 4.27969400 & -5.61166100 & -3.55003600 \\ \mathrm{H} & 2.65518400 & -2.65092700 & -3.98123000 \\ \mathrm{H} & 3.76946700 & -4.45059400 & -5.29772200 \\ \mathrm{H} & 4.77762800 & -6.39633900 & -4.11374600 \\ \mathrm{O} & 2.18940800 & -1.37885700 & -1.94927600 \\ \mathrm{O} & 3.53255300 & -4.81196700 & -0.07413600 \\ \mathrm{C} & 1.61751300 & -1.73190900 & 1.00846900 \\ \mathrm{H} & 2.13977900 & -1.79185500 & 1.97201300 \\ \mathrm{H} & 1.79404700 & -0.72446700 & 0.62996400 \\ \mathrm{C} & 0.10605000 & -1.89778000 & 1.29912400 \\ \mathrm{C} & -0.42141800 & -2.66979500 & 2.45084800 \\ \mathrm{C} & -0.85230900 & -2.16045300 & 0.25962400 \\ \mathrm{C} & -1.67138100 & -3.19933100 & 2.07828400 \\ \mathrm{~N} & -1.88947500 & -2.83725900 & 0.73403300 \\ \mathrm{C} & 0.05404700 & -2.90739300 & 3.74263700 \\ \mathrm{C} & -0.72583400 & -3.66847700 & 4.61263900 \\ \mathrm{H} & -0.36536600 & -3.86693100 & 5.61779400 \\ \mathrm{C} & -1.96903100 & -4.18782200 & 4.21484300 \\ \mathrm{H} & -2.55127800 & -4.78004700 & 4.91432400 \\ \mathrm{C} & -2.46555600 & -3.96013900 & 2.93255900 \\ \mathrm{H} & 1.01144700 & -2.50828800 & 4.06437100 \\ \mathrm{H} & -3.41967800 & -4.36844800 & 2.61491600 \\ \mathrm{H} & -2.78199100 & -2.91186100 & 0.24612500 \\ \mathrm{H} & 2.70685900 & -4.12288600 & 1.63298800 \\ \mathrm{H} & -0.84737900 & -1.75704300 & -0.74379600 \\ & & & \\ & & & \\ \mathrm{H} & & & \end{array}$


TS-II (Table S4, entry 11)

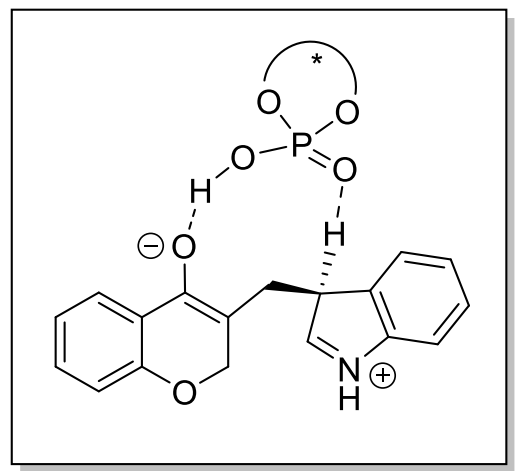

SCF energy [B3LYP-GD3BJ/6-311+G**/SMD (Chlorobenzene)]: -3291.663436190 a.u.

Thermal correction to Gibbs free energy at $258 \mathrm{~K}: 1.112228000$ a.u.

Gibbs free energy at $258 \mathrm{~K}$ [B3LYP-GD3BJ/6-311+G**/SMD (Chlorobenzene)]: -3290.551208190 a.u.

\begin{tabular}{lrrr}
01 & & & \\
$\mathrm{P}$ & -0.32067300 & 1.30732300 & -0.13929000 \\
$\mathrm{O}$ & -1.55777400 & 1.92290600 & -1.02876700 \\
$\mathrm{O}$ & 0.60609200 & 2.63698600 & 0.18455300 \\
$\mathrm{O}$ & 0.39912800 & 0.33633200 & -1.03280600 \\
$\mathrm{O}$ & -0.79564700 & 0.84113100 & 1.23095000 \\
$\mathrm{C}$ & -2.39210100 & 2.87101400 & -0.44112600 \\
$\mathrm{C}$ & -3.58636400 & 2.46460900 & 0.18900200 \\
$\mathrm{C}$ & -4.33767400 & 3.45295700 & 0.84837200 \\
$\mathrm{C}$ & -3.92077100 & 4.78233300 & 0.90847100 \\
$\mathrm{C}$ & -2.75729200 & 5.15698200 & 0.24452900 \\
$\mathrm{C}$ & -2.09795100 & 6.51735900 & 0.17365800 \\
$\mathrm{C}$ & -0.68865000 & 6.17760900 & -0.36030800 \\
$\mathrm{C}$ & -0.89577000 & 4.90489400 & -1.24901800 \\
$\mathrm{C}$ & -2.01134400 & 4.21062000 & -0.47308500 \\
$\mathrm{C}$ & -1.37721300 & 5.32936600 & -2.67777700 \\
$\mathrm{C}$ & -0.08231400 & 5.68241700 & -3.44160100 \\
$\mathrm{C}$ & 0.93492800 & 4.76481300 & -2.79870700 \\
$\mathrm{C}$ & 2.19266600 & 4.39647200 & -3.26502600 \\
$\mathrm{C}$ & 2.95657800 & 3.49944400 & -2.51763700 \\
$\mathrm{C}$ & 2.46673600 & 2.92404400 & -1.33513700 \\
$\mathrm{C}$ & 1.17380500 & 3.28300100 & -0.90851300 \\
$\mathrm{C}$ & 0.43271200 & 4.24085300 & -1.59762000 \\
$\mathrm{H}$ & -5.26913900 & 3.16133500 & 1.32463300 \\
$\mathrm{H}$ & -4.50712100 & 5.51335100 & 1.45991400 \\
$\mathrm{H}$ & -2.63483800 & 7.18602500 & -0.51432800 \\
$\mathrm{H}$ & -2.07072800 & 7.02355300 & 1.14559200 \\
& & & \\
\hline
\end{tabular}


$-0.22189200$

7. 00079300

$-0.91108700$

$-0.02927400$

5. 91878500

0. 47604000

$-2.10308400$

6. 14824900

$-2.64734200$

$-1.86269700$

4. 46968200

$-3.15320300$

0. 18981000

6. 73821900

$-3.30083000$

$-0.17092400$

5. 52182600

$-4.52217300$

2. 57966200

4. 79800400

$-4.19829700$

3. 95219700

3. 22396000

$-2.85484400$

3. 33197300

1. 98133300

$-0.56807300$

3. 81965900

0. 81659600

$-1.17550400$

3. 74262300

2. 29884300

0. 73363500

4. 71620300

$-0.03277400$

$-0.51312900$

4. 65822200

1. 49545800

1. 42495500

5. 12206300

0. 33304500

0. 78290500

$-4.07980900$

1. 06025200

0. 15757900

$-4.06239200$

0. 31270000

$-1.02824900$

$-4.64838500$

0. 48678600

1. 30246500

$-4.60549300$

$-0.97571200$

$-1.09290500$

$-5.20921400$

$-0.79595500$

1. 28167700

$-5.17575300$

$-1.50771800$

0. 07367100

5. 86064500

$-0.28345100$

1. 28931800

3. 46978000

0. 58066100

$-2.17283200$

3. 35650200

3. 20513100

1. 18370400

$-5.60810000$

$-2.49817800$

0. 03773100

$-4.63510500$

1. 05772700

2. 22589500

$-3.62419800$

0. 75810200

$-1.91394700$

5. 29810000

$-1.29590800$

$-1.18149500$

6. 81330900

$-1.08222500$

$-1.41512400$

7. 25283400

$-1.96158700$

$-1.90151200$

7. 35037300

$-0.91517500$

$-0.47483200$

6. 98979800

$-0.21369700$

$-2.05955400$

5. 09712700

$-2.53214300$

$-0.27385300$

5. 53903300

$-3.41954900$

$-0.74154300$

4. 03266400

$-2.74120700$

$-0.11557800$

5. 57206100

-2. 40922500

0. 70607600

4. 63284400

$-1.59546500$

$-2.53938800$

4. 81916900

$-0.79963600$

$-3.26894200$

3. 54904200

$-1.72695300$

$-2.44595000$

5. 04529600

$-2.52231000$

$-2.95350400$

$-4.56917300$

$-1.74782700$

$-2.42752300$

$-5.26206300$

-3. 12090600

$-2.33508900$

$-4.77910000$

$-3.77365100$

$-1.59906700$

$-5.20643700$

$-3.62618900$

$-3.30595700$

$-6.32189900$

$-3.02909100$

$-2.06974700$ 
$-3.09909600$

$-2.57905200$

$-1.98039100$

$-2.61819500$

$-2.53992100$

$-1.04288500$

$-3.05656300$

$-2.48077000$

$-5.83986400$

$-1.37123900$

$-6.97462200$

$-0.43590300$

$-7.43700500$

$-0.83308900$

$-7.75504400$

$-0.34314700$

$-6.60591400$

0. 57018300

-6. 44099500

$-2.77388700$

$-7.23698200$

$-2.76685300$

$-6.87736100$

$-3.13371800$

$-5.68379400$

$-3.50210900$

$-4.76202500$

$-1.47295400$

$-4.31065200$

$-0.50087400$

$-3.95676600$

$-2.15067000$

$-5.19787000$

$-1.86330000$

5. 21595900

1. 88388900

6. 74110100

2. 11976700

7. 26804500

1. 22142300

7. 16063400

2. 40836400

6. 95610400

2. 92161000

4. 95951200

0. 75251700

5. 40751700

$-0.19756100$

3. 88410300

0. 60169800

5. 39753300

1. 00936900

4. 57261600

3. 17033400

4. 77450600

4. 03416100

4. 98710400

3. 39265500

3. 48680500

3. 07120500

-5. 29164700

$-0.92379400$

$-4.81113700$

0. 04565600

$-6.33645200$

$-0.73730200$

$-5.28199100$

$-1.46672600$

$-0.22043000$

$-0.46010500$

0. 33664700

$-1.29098700$

$-0.04244700$

$-4.80133900$

0. 62459900

$-5.72171300$

0. 93758100

$-4.44142100$

0. 42657000

$-3.26639800$

$-0.12444600$

-3. 43013800

0. 73795400

$-7.83956800$

0. 88048000

$-4.92830900$

1. 00676700

$-6.87062600$
$-2.84957800$

$-2.12621700$

$-2.92989300$

$-3.82500200$

2. 56607900

3. 04824200

3. 96062200

2. 28438000

3. 27291200

2. 35474300

1. 60160800

3. 29370600

2. 04231300

3. 67069300

3. 89285600

3. 36238400

4. 59905300

2. 81109000

2. 69171100

2. 35306800

3. 66329900

1. 97679800

3. 83403500

3. 51817200

3. 98903900

4. 80600900

3. 36394500

2. 72155800

4. 35376800

3. 47205400

$-3.52023900$

$-3.68640900$

$-3.24535400$

$-4.47343900$

1. 52881500

$-1.70276500$

0. 34337200

$-1.74001300$

$-2.23743800$

$-1.51396500$

$-0.28573400$

$-2.02401900$

0. 94012600

$-2.43308600$ 
2. $04860200-5.48925200$

$-4.12865600$

1. 72074400

$-6.75105000$

$-3.62522300$

1. 86961300

$-3.35751200$

$-3.83188600$

$2.60293900-5.39771600$

$-5.05838200$

2. 02466200

$-7.64812400$

$-4.15841400$

0. 54983000

$-2.11233500$

$-2.21211900$

$-0.10915900$

$-5.87999100$

$-0.59975100$

$-0.72963800$

$-2.31192800$

0. 52048400

$-1.59296600$

$-2.67959300$

1. 08875800

$-1.12395900$

$-1.54140600$

$-0.14402200$

0. 22595900

$-1.62977400$

1. 53412700

0. 35952000

$-2.04520800$

2. 95399100

1. 57332100

$-1.28879400$

1. 16216400

1. 68890200

$-1.80078600$

3. 35214500

2. 37713400

$-1.33541300$

2. 21497200

$-0.54301800$

$-2.53760900$

3. 89902300

$-0.09262900$

$-2.77203400$

5. 19803500

$-0.78407800$

$-3.16065600$

5. 94005500

1. 23698200

$-2.51208100$

5. 56918600

1. 55562100

$-2.70088500$

6. 58994500

2. $15588600-2.01591600$

4. 64623800

$-1.57743300$

$-2.73061300$

3. 63116800

3. $18541000-1.81254900$

4. 92421800

3. 33055300

$-0.98239300$

2. 19556900

$-0.88647700$

$-4.96582400$

1. 02147300

1.91682800

$-0.91991900$

0. 20507500 
INT-III (Table S4, entry 12)

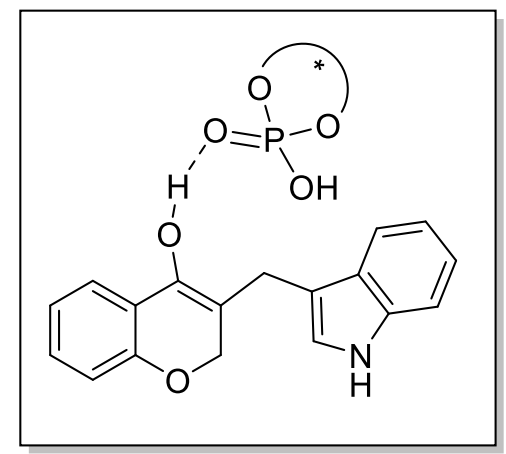

SCF energy [B3LYP-GD3BJ/6-311+G**/SMD (Chlorobenzene)]: -3291.683006080 a.u.

Thermal correction to Gibbs free energy at $258 \mathrm{~K}: 1.113197000$ a.u.

Gibbs free energy at $258 \mathrm{~K}$ [B3LYP-GD3BJ/6-311+G**/SMD (Chlorobenzene)]: -3290.569809080 a.u.

$\begin{array}{lrrr}01 & & & \\ \mathrm{P} & -0.13727700 & 1.42587300 & -0.23984600 \\ \mathrm{O} & -1.32753600 & 2.08591100 & -1.10813100 \\ \mathrm{O} & 0.80058000 & 2.67169200 & 0.21024700 \\ \mathrm{O} & 0.52636500 & 0.35023400 & -1.01530100 \\ \mathrm{O} & -0.76150800 & 1.01223900 & 1.16475900 \\ \mathrm{C} & -2.12946000 & 3.10151500 & -0.55886300 \\ \mathrm{C} & -3.36493900 & 2.75971400 & 0.02407900 \\ \mathrm{C} & -4.09037700 & 3.79484200 & 0.63869200 \\ \mathrm{C} & -3.60638500 & 5.10094600 & 0.69873000 \\ \mathrm{C} & -2.39558900 & 5.40560800 & 0.08590000 \\ \mathrm{C} & -1.66250600 & 6.72811300 & 0.03894800 \\ \mathrm{C} & -0.24658400 & 6.30730500 & -0.41044600 \\ \mathrm{C} & -0.47272800 & 5.04024200 & -1.30373600 \\ \mathrm{C} & -1.66641900 & 4.41361000 & -0.58816300 \\ \mathrm{C} & -0.83895900 & 5.47997200 & -2.76148100 \\ \mathrm{C} & 0.52046000 & 5.76555400 & -3.43760200 \\ \mathrm{C} & 1.44783000 & 4.80384300 & -2.72628700 \\ \mathrm{C} & 2.71700000 & 4.37508600 & -3.10117800 \\ \mathrm{C} & 3.38526000 & 3.44368400 & -2.30473900 \\ \mathrm{C} & 2.78715400 & 2.88784500 & -1.16363200 \\ \mathrm{C} & 1.48837400 & 3.31399300 & -0.83730600 \\ \mathrm{C} & 0.83744100 & 4.30503400 & -1.56466100 \\ \mathrm{H} & -5.05266400 & 3.55608700 & 1.08145300 \\ \mathrm{H} & -4.17580000 & 5.86957600 & 1.21528300 \\ \mathrm{H} & -2.12448100 & 7.41426300 & -0.68492700 \\ \mathrm{H} & -1.66322500 & 7.24405900 & 1.00578300\end{array}$




\begin{tabular}{|c|c|c|c|}
\hline H & 0.29614000 & 7. 09767800 & -0.93833300 \\
\hline H & 0.34833300 & 6. 02047600 & 0.46415200 \\
\hline & -1.52292800 & 6. 33421400 & -2.77937800 \\
\hline & -1.33484900 & 4. 64400900 & -3.26760600 \\
\hline & 0.83285000 & 6. 80792800 & -3.28328500 \\
\hline & 0.49567800 & 5. 60156800 & -4.52074600 \\
\hline & 3. 18705000 & 4. 75654700 & -4.00410000 \\
\hline & 4. 38713700 & 3. 11967400 & -2.57158600 \\
\hline & 3. 52893500 & 1. 89322200 & -0.33551200 \\
\hline & 3. 94186700 & 0.67698900 & -0.89135200 \\
\hline & 3. 86985800 & 2. 19961400 & 0.98819800 \\
\hline & 4. 68712900 & -0.24592200 & -0.14583500 \\
\hline & 4. 63299900 & 1. 31728500 & 1. 76148900 \\
\hline & 5. 01996200 & 0.10270600 & 1. 17254800 \\
\hline & -3.91562200 & 1. 37663600 & 0.00709100 \\
\hline & -3.92393200 & 0.61244500 & -1.16753300 \\
\hline & -4.49758900 & 0.84272700 & 1. 16404200 \\
\hline & -4.50381900 & -0.66112300 & -1.20788900 \\
\hline & -5.08717800 & -0.42693700 & 1. $1700050 \mathrm{c}$ \\
\hline & -5.07662900 & -1.15851700 & -0.02708200 \\
\hline & 5. 61662000 & -0.59236900 & 1. 75683300 \\
\hline & 3. 64787800 & 0.45391900 & $-1.9095060 c$ \\
\hline & 3. 54640000 & 3. 15059300 & 1. 39460300 \\
\hline & -5.53071700 & -2.13974400 & -0.04317400 \\
\hline & -4.47030900 & 1. 43233700 & 2. 07527500 \\
\hline & -3.48120000 & 1. 03204900 & -2.06406100 \\
\hline & 5. 16312900 & -1.58844200 & -0.73675900 \\
\hline & 6. 70920700 & -1.58699200 & -0.80699900 \\
\hline & 7. 07219300 & -2.53146700 & -1.23034300 \\
\hline & 7. 16173100 & -1.46843100 & 0.18378400 \\
\hline & 7. 07275600 & -0.76919900 & -1.43986800 \\
\hline & 4. 69665500 & -2.76095100 & 0.15804600 \\
\hline & 5. 05270600 & -3.71293900 & $-0.2532180 c$ \\
\hline & 3. 60343800 & -2.80793800 & $0.2105330 \mathrm{C}$ \\
\hline & 5. 08142300 & -2.67961800 & 1. 18049400 \\
\hline & 4. 61112200 & -1.82769200 & -2.15550200 \\
\hline & 4. 97183000 & -1.07434100 & -2.86547700 \\
\hline & 3. 51580400 & -1.82267500 & -2.17581900 \\
\hline & 4. 94317300 & -2.80645500 & -2.51906900 \\
\hline & -4.51416800 & -1.44768100 & -2.53439800 \\
\hline & -5.19688700 & -2.82247200 & -2.40104700 \\
\hline & -4.68753500 & -3.46222100 & -1.67143600 \\
\hline & -5.16871800 & -3.34073000 & -3.36600900 \\
\hline & -6.24873200 & -2.73312900 & $-2.1050540 \mathrm{C}$ \\
\hline
\end{tabular}


$-3.06324000$

$-2.50606200$

$-1.67940900$

-2. 30712800

$-2.51204800$

$-0.74114700$

$-3.06361600$

$-2.18964800$

$-5.73628100$

$-0.95783200$

$-6.91689600$

$-0.03533700$

$-7.39553900$

$-0.39394300$

$-7.67453900$

$-0.01298100$

$-6.58589900$

0. 99381000

$-6.28243600$

$-2.38966800$

$-7.06811000$

-2. 44773800

$-6.71876900$

$-2.72331300$

$-5.49282200$

$-3.10062700$

$-4.69900100$

$-0.96489200$

$-4.30426500$

0. 03589500

$-3.84845700$

$-1.61472100$

$-5.16036600$

$-1.33181200$

5. 07574400

1. 64970200

6. 62017700

1. 61133900

7. 02182500

0. 62590800

6. 95135700

1. 85041300

7. 06701000

2. 34229600

4. 48596900

0. 61074900

4. 78946300

$-0.41091300$

3. 38978900

0. 66430200

4. 82713100

0. 80769800

4. 60783400

3. 04630300

5. 01707400

3. 83748700

4. 95086600

3. 23614400

3. 51561200

3. 13426700

$-5.28523600$

$-0.63328800$

$-4.81555200$

0. 33731300

$-6.31841600$

$-0.44901300$

$-5.31276600$

$-1.18156600$

$-0.47547400$

0. 10714300

0. 47156900

$-1.32327900$

$-0.11092900$

$-4.94784600$

0. 61315600

$-5.72252400$

0. 97843700

$-4.41589900$

0. 46484300

$-3.28962200$

$-0.14203200$

$-3.53015500$

0. 68677900

$-7.81362900$

0.78814400

$-5.13702500$

0. 99554500

$-6.82330300$
$-3.01814100$

$-2.31450100$

$-3.13686100$

$-3.98905400$

2. 46405100

2. 85308600

3. 77279900

2. 06137500

3. 02854400

2. 30631700

1. 54454700

3. 25462000

2. 03723600

3. 61161000

3. 81430900

3. 37802100

4. 53695100

3. 20127200

3. 28863900

3. 03124900

4. 30667600

2. 60551700

4. 18398700

3. 92694900

4. 18705900

5. 20756800

3. 65388300

3. 01600300

4. 67723000

3. 64973900

$-3.60132900$

$-3.79189900$

$-3.28442900$

$-4.55099400$

1. 43236500

$-1.80699100$

$-0.08345200$

$-2.20577200$

$-2.58581300$

$-1.79187400$

$-0.60653100$

$-2.65288600$

0. 52921200

$-2.97294000$ 
$1.74322600-4.24470400$

$-3.74745600$

C

2. $14180100-5.34237300$

$-4.50907800$

C

$\begin{array}{lll}1.76207000 & -6.63028200 & -4.12210700\end{array}$

$\mathrm{H}$

2. $00592000-3.23692100$

$-4.04936900$

2. $73792000-5.19324400$

$-5.40498600$

2. 06676600

$-7.49069100$

$-4.71235000$

0.63230000

$-2.08488200$

$-2.40217800$

$-0.17025200$

$-5.94805300$

$-1.11162600$

$-0.77979000$

$-2.48210700$

0. 27761000

$-1.78456900$

$-2.82201800$

0. 56508700

$-0.93753400-1.56623600$

$-0.29803500$

$-0.00279100$

$-2.17608500$

1. 54495500

$-0.29837300$

$-2.64488600$

2. 88037900

$1.19642300-1.49606200$

1. 63544600

0. 75821000

$-2.20768200$

3. 72741200

1. 65191600

$-1.51579100$

2. 93800200

$-1.34872600$

$-3.39382100$

3. 44242300

$-1.32640700$

$-3.67568200$

4. 80211500

$-2.13252400$

$-4.25452100$

5. 24420900

$-0.27161500$

$-3.22370300$

5. 62261600

$-0.28233500$

$-3.45925200$

6. 68322500

0.78181200

$-2.48463300$

5. 09915700

$-2.16656200-3.75073800$

2. 82156200

$1.59536900-2.13807800$

5. 73100700

$2.50184000-1.07217500$

3. 25234800

$-0.98021800$

$-5.14471300$

0. 55236000

1. 77953900

$-1.02259500$

0. 85752600 
TS-III-R (Table S4, entry 13)

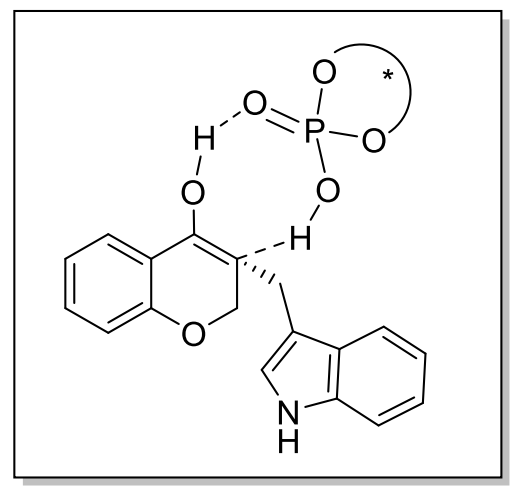

SCF energy [B3LYP-GD3BJ/6-311+G**/SMD (Chlorobenzene)]: -3291.662756180 a.u.

Thermal correction to Gibbs free energy at $258 \mathrm{~K}: 1.109545000$ a.u.

Gibbs free energy at $258 \mathrm{~K}$ [B3LYP-GD3BJ/6-311+G**/SMD (Chlorobenzene)]: -3290.553211180 a.u.

\begin{tabular}{|c|c|c|c|}
\hline $\mathrm{P}$ & 0.41070400 & 1. 35328900 & 0.03919100 \\
\hline 0 & -0.50880300 & 2. 51315400 & -0.66767100 \\
\hline 0 & 1. 76313600 & 2. 15346500 & 0.49249700 \\
\hline 0 & 0.75819000 & 0.37472100 & -1.10317000 \\
\hline 0 & -0.23473900 & 0.78127000 & 1. 26807800 \\
\hline $\mathrm{C}$ & -0.89060400 & 3. 59079000 & 0.14583300 \\
\hline $\mathrm{C}$ & -2.10561600 & 3. 54708900 & 0.85506800 \\
\hline $\mathrm{C}$ & -2.38831900 & 4. 60020900 & 1. 74067200 \\
\hline $\mathrm{C}$ & -1.50261000 & 5. 65844200 & 1. 93473500 \\
\hline $\mathrm{C}$ & -0.32561000 & 5. 69248100 & 1. 19653900 \\
\hline $\mathrm{C}$ & 0.79468700 & 6. 70730300 & 1. 25320300 \\
\hline $\mathrm{C}$ & 1. 95039100 & 5. 96004200 & 0.55680000 \\
\hline $\mathrm{C}$ & 1. 24787100 & 5. 02126300 & -0.48336300 \\
\hline $\mathrm{C}$ & -0.02772300 & 4. 67794500 & 0.27345600 \\
\hline $\mathrm{C}$ & 0.95212900 & 5. 81955300 & -1.79593300 \\
\hline $\mathrm{C}$ & 2. 28594700 & 5. 79346500 & -2.57446900 \\
\hline $\mathrm{C}$ & 2. 90264400 & 4. 48664000 & -2.12518800 \\
\hline $\mathrm{C}$ & 3. 98116000 & 3. 79843100 & -2.66820600 \\
\hline $\mathrm{C}$ & 4. 37904400 & 2. 59862500 & -2.08304100 \\
\hline $\mathrm{C}$ & 3. 68112700 & 2. 01649600 & -1.00836700 \\
\hline $\mathrm{C}$ & 2. 56463800 & 2. 72068200 & -0.50915600 \\
\hline C & 2. 22469000 & 3. 97660900 & -1.00802900 \\
\hline $\mathrm{H}$ & 0.39615000 & -0.78807500 & -0.86395600 \\
\hline $\mathrm{H}$ & -3.32445000 & 4. 56861200 & 2. 29075800 \\
\hline $\mathrm{H}$ & -1.73264200 & 6. 44108400 & 2. 65351100 \\
\hline
\end{tabular}




\begin{tabular}{|c|c|c|c|}
\hline $\mathrm{H}$ & 0.52752500 & 7. 62537300 & 0.71045700 \\
\hline H & 1. 04049600 & 7. 00761900 & 2.27820700 \\
\hline & 2. 68321600 & 6. 62470800 & 0.08857900 \\
\hline & 2. 48099200 & 5. 33881000 & 1. 28710500 \\
\hline & 0.58401100 & 6. 83072400 & -1.59473100 \\
\hline & 0.17964900 & 5. 28942400 & -2.36451800 \\
\hline & 2. 92276800 & 6. 64869000 & -2.30710200 \\
\hline & 2. 14216900 & 5. 83794000 & -3.66032700 \\
\hline & 4. 52095000 & 4. 19332600 & -3.52547200 \\
\hline & 5. 25684700 & 2. 08616500 & -2.46356000 \\
\hline & 4. 17334500 & 0.73167100 & -0.44181100 \\
\hline & 4. 71427100 & -0.24153000 & -1.29568300 \\
\hline & 4. 20751400 & 0.51152200 & 0.94320000 \\
\hline & 5. 30556500 & -1.40656400 & $-0.7986430 c$ \\
\hline & 4. 77087200 & -0.65039800 & 1. $4812850 \mathrm{C}$ \\
\hline & 5. 31412400 & -1.58857100 & 0.59150000 \\
\hline & -3.11234400 & 2. 45840900 & 0.70902500 \\
\hline & -3.75133300 & 2. 23432900 & -0.51148100 \\
\hline & -3.51574400 & 1. 73269500 & 1. 83995800 \\
\hline & -4.79756100 & 1. 30472400 & -0.62705300 \\
\hline & -4.53914500 & 0.78615100 & 1. 76674700 \\
\hline & -5.15999700 & 0.58788100 & 0.51937400 \\
\hline & 0.28815100 & -2.98891900 & -1.78712300 \\
\hline & 1. 44462800 & -4.14787900 & -0.08591600 \\
\hline & 0.63984300 & -3.56195800 & 0.91490200 \\
\hline & -0.29722700 & -1.99910900 & -0.78342200 \\
\hline & 2. 87336100 & -5.67218200 & -0.56468900 \\
\hline & -0.43587200 & -3.77885700 & -2.02895700 \\
\hline & 2. 26071500 & -5.24154700 & 0.21960700 \\
\hline & 0.67582400 & -4.08396500 & 2.22185200 \\
\hline$C$ & 1. 46791500 & -5.18368200 & 2.51787400 \\
\hline & 2. 26250200 & -5.75498300 & 1. 51259300 \\
\hline & 0.06903500 & -3.60978800 & 2. 98541900 \\
\hline & 1. 47945600 & -5.59403700 & 3. 52279200 \\
\hline & 2. 88801700 & -6.61371300 & 1. 74160100 \\
\hline & -0.65934500 & -1.72672900 & 1. 58295400 \\
\hline o & 1. 49900200 & -3.62859000 & -1.33541500 \\
\hline$C$ & -1.65802300 & -1.39864500 & -1.24394800 \\
\hline & -1.46227800 & -0.62204500 & -1.99020600 \\
\hline & -2.12245000 & -0.88816200 & -0.39367900 \\
\hline & -2.60289800 & -2.41137100 & -1.83361300 \\
\hline & -3.30978500 & -3.47801800 & -1.16115900 \\
\hline & -2.93429200 & -2.51394500 & -3.16432000 \\
\hline & -4.05692400 & -4.17894100 & $-2.1488340 \mathrm{C}$ \\
\hline
\end{tabular}


$-3.80354500$

$-3.56884700$

$-3.91215500$

$-3.38453700$

$-5.00397500$

$-4.18568000$

-4. 24950900

$-5.34498400$

$-5.67854300$

$-4.92022700$

$-5.53910200$

$-4.86711100$

$-5.27595700$

$-2.83087300$

$-3.39726700$

$-5.43282700$

$-4.21847200$

$-5.79587800$

$-3.82447400$

0. 56523100

$-2.48281700$

$-2.60946600$

$-1.90696400$

$-0.72029200$

$-0.69150400$

2. 82489900

$-5.95732000$

$-0.14315300$

$-3.00016600$

1. 92157100

3. 79828700

1. 27026100

5. 76292000

$-2.49123200$

4. 65850500

-0. 07490900

$-5.56185500$

1. 17518200

2. 46553400

2. 61164700

$-7.10696600$

$-6.95269700$

2. 41005500

3. 35154400

$-5.74783000$

$-6.52511400$

$-7.31847400$

$-5.99732500$

$-7.01175300$

$-4.57932600$

$-3.98635800$

$-3.88864300$

$-5.13442200$

4. 82418700

5. 95295500

6. 29598700

6. 78528900

6. 34806100

6. 87621500

4. 04014600

4. 44604500

2. 98754600

4. 08222400

4. 20400300

3. 14951600
$-0.02794700$

0. 07439500

$-0.97025700$

-0. 10181400

1. 00637700

0. 09377100

1. 85050600

0. 93404400

$-0.91349100$

$-2.46202300$

$-1.08033700$

$-1.91973600$

$-1.26638500$

$-0.17596700$

$-2.20644500$

$-3.07611700$

$-2.10776400$

$-2.41762200$

0. 23651300

0.39429600
-3. 36125200

0. 17452200

0. 48658800

1. 51616900

$-0.50970800$

$-0.23456600$

$-1.83913200$

0. 95338300

$-2.60811800$

$-4.24350900$

$-2.71522500$

$-3.99888800$

1. 40760900

$-1.36983100$

0. 45188500

2. 77361500

1. 59604100

0. 99575300

$-2.36442100$

$-1.96008600$

$-2.17479200$

$-1.35819400$

$-3.11582700$

$-2.21725300$

$-1.97084800$

$-1.22216300$

$-1.78564000$

$-2.95046600$

$-3.14048500$

$-3.02280300$

$-3.23040500$

$-4.08422500$

2. 99937300

$-1.71731700$

3. 44631100

2. 94086300

4. 52636300

3. 22960400

3. 32442700

2. 79707900

3. 03826600

4. 40052800

3. 81622600

3. 56451200 
$4.25882600-0.00063900$

4. 88503300

7. $44894700 \quad-2.60972600$

$-1.35037600$

7. 92830100

$-3.35858300$

$-1.99330100$

7. $58491100 \quad-2.92669600$

$-0.31113500$

7. 98017300

$-1.65974200$

$-1.48036000$

5. 86483300

$-2.07949500$

$-3.20724000$

6. 37701100

$-1.13360000$

$-3.41761100$

4. 82605200

$-1.98842600$

$-3.54382700$

6. 34248200

$-2.85604300$

$-3.81583400$

$5.24199200-3.82186300$

$-1.53244100$

5. 30019300

-4. 17144900

$-0.49619000$

5. 70629200

$-4.58523900$

$-2.17004100$

4. 18322600

$-3.74458600$

$-1.80124500$

$-0.14990900$

$-2.39258000$

0. 56372600

$-5.00125300$

$-0.02462000$

2. 99373100

$-4.69323800$

$-1.52180300$

2. 76206900

$-5.18592900$

$-1.90802200$

1. 86400000

$-5.03363900$

$-2.12042000$

3. 61673000

$-3.61486000$

$-1.67849200$

2. 64464800

$-4.28897800$

0. 41456000

4. 28785200

$-4.47315900$

1. 47070600

4. 51615500

$-3.20577600$

0. 26100800

4. 23123400

$-4.66186500$

$-0.17709100$

5. 13184500

$-6.52268600$

0. 16230700

3. 20258500

$-6.85836000$

$-0.40631600$

4. 07859400

$-7.10223300$

-0. 18533600

2. 34139600

$-6.76888900$

1. 21756100

3. 36824300 
TS-III-S (Table S4, entry 14)<smiles>O=P12OCCCC(CO1)c1c2o[pH]oc2ccccc12</smiles>

SCF energy [B3LYP-GD3BJ/6-311+G**/SMD (Chlorobenzene)]: -3291.667743360 a.u.

Thermal correction to Gibbs free energy at $258 \mathrm{~K}: 1.111076000$ a.u.

Gibbs free energy at $258 \mathrm{~K}$ [B3LYP-GD3BJ/6-311+G**/SMD (Chlorobenzene)]: -3290.556667360 a.u.

\begin{tabular}{|c|c|c|c|}
\hline $\mathrm{P}$ & 0.12593400 & 1. 39840000 & -0.10424600 \\
\hline 0 & 1. 29776300 & 2. 39231800 & 0.46198000 \\
\hline 0 & -1.01669200 & 2. 41702300 & -0.68316300 \\
\hline 0 & -0.39616200 & 0.69141600 & 1. 16804600 \\
\hline 0 & 0.57286500 & 0.52390100 & -1.23932400 \\
\hline $\mathrm{C}$ & 1.95459600 & 3. 18333700 & -0.49136500 \\
\hline $\mathrm{C}$ & 3. 14690800 & 2. 72694500 & -1.08572500 \\
\hline $\mathrm{C}$ & 3. 68684000 & 3. 49666000 & -2.13040100 \\
\hline $\mathrm{C}$ & 3. 07352900 & 4. 66488700 & -2.58137300 \\
\hline C & 1.92588100 & 5. 11589000 & -1.93843900 \\
\hline $\mathrm{C}$ & 1. 08258000 & 6. 33602000 & -2.23888700 \\
\hline C & -0.21593400 & 6. 04607500 & -1.45385900 \\
\hline $\mathrm{C}$ & 0.24317100 & 5. 18221700 & -0.23084400 \\
\hline $\mathrm{C}$ & 1. 38404800 & 4. 39738200 & -0.86231200 \\
\hline $\mathrm{C}$ & 0.76443500 & 6. 11562600 & 0.91326100 \\
\hline $\mathrm{C}$ & -0.50646100 & 6. 55793100 & 1. 66982900 \\
\hline $\mathrm{C}$ & -1.43271700 & 5. 37837000 & 1. 47110300 \\
\hline $\mathrm{C}$ & -2.61232900 & 5. 07547100 & 2. 14099000 \\
\hline $\mathrm{C}$ & -3.31375600 & 3. 92514000 & 1. 78625600 \\
\hline $\mathrm{C}$ & -2.83261500 & 3. 02148800 & 0.82162100 \\
\hline $\mathrm{C}$ & -1.60407300 & 3. 33042700 & 0.20115500 \\
\hline C & -0.94622500 & 4. 53027500 & 0.46480500 \\
\hline $\mathrm{H}$ & -0.42313300 & -0.51344700 & 1. 02665400 \\
\hline $\mathrm{H}$ & 4. 60940400 & 3. 15866700 & -2.59322400 \\
\hline $\mathrm{H}$ & 3. 49724800 & 5. 21658000 & -3.41690200 \\
\hline
\end{tabular}




\begin{tabular}{|c|c|c|c|}
\hline $\mathrm{H}$ & 1. 56920900 & 7. 25418600 & -1.88012200 \\
\hline H & 0.90565200 & 6. 47274400 & -3.3118640 \\
\hline H & -0.75175700 & 6.95035800 & -1.14797300 \\
\hline $\mathrm{H}$ & -0.89539500 & 5. 44788100 & -2.0714820 \\
\hline $\mathrm{H}$ & 1. 35768600 & 6. 95207600 & 0.530268 \\
\hline $\mathrm{H}$ & 1. 40621000 & 5. 52863000 & 1. 579953 \\
\hline H & -0.93208300 & 7. 47462300 & 1. 237222 \\
\hline H & -0.31620400 & 6. 76980100 & 2. 7283210 \\
\hline $\mathrm{H}$ & -2.99659600 & 5.73183900 & 2. 917884 \\
\hline H & -4.26726000 & 3. 71280100 & 2.260101 \\
\hline $\mathrm{C}$ & -3.67191500 & 1. 84550300 & 0.4620460 \\
\hline $\mathrm{C}$ & -4.35967300 & 1. 14183000 & 1. 462380 \\
\hline $\mathrm{C}$ & -3.90126900 & 1. 51359500 & -0.881614 \\
\hline $\mathrm{C}$ & -5.29461100 & 0.14930500 & 1. 147681 \\
\hline $\mathrm{C}$ & -4.82459600 & 0.52514100 & -1.240952 \\
\hline $\mathrm{C}$ & -5.51046800 & -0.13454600 & -0.209255 \\
\hline $\mathrm{C}$ & 3. 87454200 & 1.51291700 & -0.626409 \\
\hline $\mathrm{C}$ & 4. 17861300 & 1. 33703500 & 0.725694 \\
\hline $\mathrm{C}$ & 4. 36261900 & 0.58571000 & -1.561774 \\
\hline $\mathrm{C}$ & 4. 98192100 & 0.27362900 & 1. 1639630 \\
\hline $\mathrm{C}$ & 5. 15650800 & -0.49288100 & -1.169044 \\
\hline $\mathrm{C}$ & 5. 45914300 & -0.62140000 & 0.199898 \\
\hline $\mathrm{C}$ & -1.08687000 & -2.45828100 & 2. 098831 \\
\hline C & 0.71515400 & -3.94910900 & 2. 338134 \\
\hline $\mathrm{C}$ & 1. 51188600 & -3.12070100 & 1.509650 \\
\hline $\mathrm{C}$ & 0.82605700 & -2.22566900 & 0.596678 \\
\hline $\mathrm{C}$ & -0.53280700 & -1.90755400 & 0.793077 \\
\hline H & 0.67121100 & -5.62830100 & 3. 670007 \\
\hline H & -0.81812200 & -1.80953300 & 2. 944316 \\
\hline $\mathrm{C}$ & 1. 30535100 & -4.99453800 & 3. 058966 \\
\hline $\mathrm{C}$ & 2. 90516800 & -3.32809600 & 1. 4658260 \\
\hline $\mathrm{C}$ & 3. 48917100 & -4.34292000 & 2. 208162 \\
\hline $\mathrm{C}$ & 2. 68070900 & -5.18069300 & 2. 993877 \\
\hline H & 3. 50246900 & -2.68055500 & 0.8335690 \\
\hline $\mathrm{H}$ & 4. 56319300 & -4.49829200 & 2.1735860 \\
\hline H & 3. 13494200 & -5.98286800 & 3.569660 \\
\hline 0 & 1. 54419100 & -1.77767400 & -0.4183680 \\
\hline 0 & -0.62099100 & -3.78782200 & 2. 4323420 \\
\hline $\mathrm{C}$ & -1.49106900 & -1.90140400 & -0.4288230 \\
\hline H & -0.97812800 & -1.45050700 & -1.2830570 \\
\hline H & -2.33246600 & -1.23642200 & -0.205259 \\
\hline C & -2.01643000 & -3.26617100 & -0.7813440 \\
\hline $\mathrm{C}$ & -1.31768800 & -4.34738600 & -1.4408130 \\
\hline & -3.27906700 & -3.73568600 & -0.509482 \\
\hline
\end{tabular}




\begin{tabular}{rrr}
-2.22421000 & -5.44045800 & -1.53212100 \\
-3.41005800 & -5.03772600 & -0.95177100 \\
-0.02658300 & -4.49798100 & -1.97812100 \\
0.32157300 & -5.70402800 & -2.57460600 \\
1.31736500 & -5.82718500 & -2.99177200 \\
-0.59353300 & -6.77317700 & -2.64885800 \\
-0.29184000 & -7.70432100 & -3.12098100 \\
-1.87809800 & -6.65591700 & -2.13039100 \\
0.69094100 & -3.68436600 & -1.92902600 \\
-2.58701400 & -7.47805000 & -2.19028300 \\
-4.24725800 & -5.59454300 & -0.88518400 \\
-2.17349500 & -2.54503200 & 2.05730700 \\
-4.10916700 & -3.22772700 & -0.03879100 \\
1.13459100 & -0.95192100 & -0.84309300 \\
-6.25094100 & -0.88387800 & -0.47435100 \\
-4.15357900 & 1.39007500 & 2.49665700 \\
-3.36638800 & 2.06312700 & -1.64497000 \\
6.09042300 & -1.44496700 & 0.51350400 \\
4.09515200 & 0.72312800 & -2.60272200 \\
3.80163100 & 2.06070400 & 1.44038400 \\
-6.11398400 & -0.58772100 & 2.22724400 \\
-7.61353500 & -0.25332700 & 2.03923500 \\
-8.21892000 & -0.76660200 & 2.79674300 \\
-7.97806900 & -0.56177400 & 1.05365000 \\
-7.78849700 & 0.82404500 & 2.13642400 \\
-5.91842700 & -2.11613200 & 2.09744000 \\
-6.50924100 & -2.64364200 & 2.85638000 \\
-4.86680900 & -2.39187300 & 2.24005800 \\
-6.23607800 & -2.48717400 & 1.11679500 \\
-5.70767400 & -0.17945700 & 3.65627900 \\
-5.87961700 & 0.88676300 & 3.84023200 \\
-4.65214800 & -0.39437800 & 3.85779700 \\
-6.30564700 & -0.73919400 & 4.38445300 \\
5.32808200 & 0.15840200 & 2.66195800 \\
6.21069000 & -1.06498200 & 2.97474900 \\
5.71175000 & -2.00576600 & 2.71574800 \\
6.42957100 & -1.09512200 & 4.04825800 \\
\hline .16917400 & -1.02539200 & 2.44444500 \\
\hline & -0.03649700 & 3.48880300 \\
\hline
\end{tabular}


$-1.13302500$

$\begin{array}{lll}7.64783200 & -0.52523400 & -2.40085100\end{array}$

$5.19442200-2.93458800$

$-1.80526800$

$5.53832600-3.24726600$

$-0.81320800$

5. $55802000-3.67495200$

$-2.52872700$

4. 09919900

$-2.96319300$

$-1.80479100$

$5.27110200-1.22316600$

$-3.62074400$

5. 62816700

$-0.24463600$

$-3.96160800$

4. $18125600-1.24666100$

$-3.73051400$

5. 68809900

$-1.97933500$

$-4.29572300$

$-5.15162700$

0. 20004000

$-2.71261200$

$-5.02641200$

$-1.31962400$

$-2.96912100$

$-4.01330000$

$-1.67787700$

$-2.75843600$

$-5.25618300$

$-1.54476600$

$-4.01765000$

$-5.71992300$

$-1.89962400$

$-2.35074600$

$-4.21069500$

0. 92463100

$-3.69472000$

$-3.16060700$

0. 66830200

$-3.51644900$

$-4.31159200$

2. 01385900

$-3.63155600$

$-4.45630000$

0. 63261900

$-4.72212100$

$-6.60325700$

0. 64703300

$-3.01135700$

$-7.32554700$

0. 13154100

$-2.36865500$

$-6.86518500$

0. 42771200

$-4.05407600$

$-6.72265600$

1. 72453600

$-2.85001200$

6. 09707900

1. 42626300

3. 10570200

5. 50373500

2. 33442000

2. 95809100

7. 02798800

1. 53906400

2. 53803200

6. 35283300

1. 36414800

4. 17080000 
INT-IV-R (Table S4, entry 15)

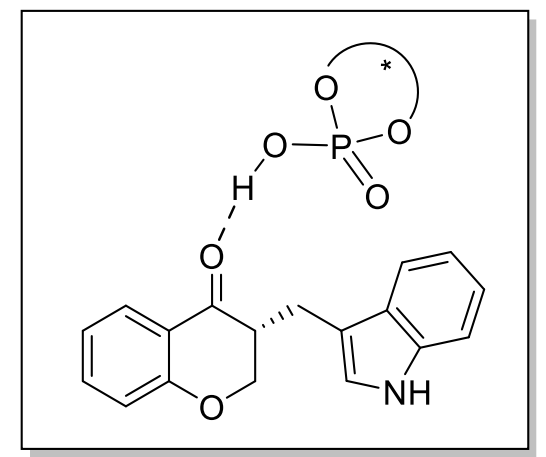

SCF energy [B3LYP-GD3BJ/6-311+G**/SMD (Chlorobenzene)]: -3291.697307340 a.u.

Thermal correction to Gibbs free energy at $258 \mathrm{~K}: 1.113018000$ a.u.

Gibbs free energy at $258 \mathrm{~K}$ [B3LYP-GD3BJ/6-311+G**/SMD (Chlorobenzene)]: -3290.584289340 a.u.

01

\begin{tabular}{lrrr}
$\mathrm{P}$ & 0.37044500 & 1.45282300 & -0.10056200 \\
0 & -0.63017500 & 2.65544500 & -0.55485400 \\
$\mathrm{O}$ & 1.63359900 & 2.22618000 & 0.59319600 \\
$\mathrm{O}$ & 0.69427600 & 0.57936400 & -1.24872100 \\
$\mathrm{O}$ & -0.30658100 & 0.79815200 & 1.17417600 \\
$\mathrm{C}$ & -1.10345700 & 3.56888600 & 0.39650300 \\
$\mathrm{C}$ & -2.37456000 & 3.37886600 & 0.97553900 \\
$\mathrm{C}$ & -2.75102600 & 4.26040000 & 2.00448000 \\
$\mathrm{C}$ & -1.91092800 & 5.27744300 & 2.45393200 \\
$\mathrm{C}$ & -0.68809900 & 5.47161900 & 1.82170000 \\
$\mathrm{C}$ & 0.38043700 & 6.50063000 & 2.11878300 \\
$\mathrm{C}$ & 1.59838800 & 5.94897500 & 1.34710100 \\
$\mathrm{C}$ & 0.98380900 & 5.18181300 & 0.12756900 \\
$\mathrm{C}$ & -0.29630700 & 4.64484900 & 0.75786500 \\
$\mathrm{C}$ & 0.67153900 & 6.18940800 & -1.02995200 \\
$\mathrm{C}$ & 2.01609100 & 6.36586200 & -1.76991900 \\
$\mathrm{C}$ & 2.68625100 & 5.02589500 & -1.55498300 \\
$\mathrm{C}$ & 3.78308400 & 4.47866000 & -2.21179600 \\
$\mathrm{C}$ & 4.22802900 & 3.20864800 & -1.84576300 \\
$\mathrm{C}$ & 3.56253200 & 2.43453100 & -0.87940200 \\
$\mathrm{C}$ & 2.42211100 & 2.99763200 & -0.27380100 \\
$\mathrm{C}$ & 2.02620600 & 4.30275100 & -0.55026100 \\
$\mathrm{H}$ & 0.86287900 & -1.79968400 & -1.20863000 \\
$\mathrm{H}$ & -3.73217200 & 4.13808600 & 2.45311900 \\
$\mathrm{H}$ & -2.22041600 & 5.91776100 & 3.27631500 \\
$\mathrm{H}$ & 0.08905900 & 7.49424700 & 1.74920000 \\
& & & \\
\hline
\end{tabular}




\begin{tabular}{|c|c|c|c|}
\hline $\mathrm{H}$ & 0.57415100 & 6. 60945100 & 3. 19214700 \\
\hline $\mathrm{H}$ & 2. 30689800 & 6.72422200 & 1. 03832500 \\
\hline $\mathrm{H}$ & 2. 14050300 & 5. 23291400 & 1. 97510000 \\
\hline $\mathrm{H}$ & 0.24943600 & 7. 12995300 & -0.66177900 \\
\hline $\mathrm{H}$ & -0.06368800 & 5. 73384800 & -1.70294600 \\
\hline H & 2. 60863000 & 7. 18221600 & -1.33298600 \\
\hline H & 1. 88541700 & 6. 60621100 & -2.83134400 \\
\hline & 4. 29908600 & 5. 03386300 & -2.99130100 \\
\hline H & 5. 11454500 & 2. 79281300 & -2.31547800 \\
\hline & 4. 11221100 & 1. 10502600 & -0.50053100 \\
\hline & 4. 54481400 & 0.20901300 & -1.48714900 \\
\hline $\mathrm{C}$ & 4. 30712000 & 0.78203400 & 0.84974300 \\
\hline C & 5. 18118700 & -0.99192600 & -1.15153400 \\
\hline $\mathrm{C}$ & 4. 94303800 & -0.40416600 & 1. 22810400 \\
\hline C & 5. 37084400 & -1.26910100 & 0.20979400 \\
\hline C & -3.34527100 & 2. 34848400 & 0.51540500 \\
\hline C & -3.59138700 & 2. 15323700 & -0.84691900 \\
\hline $\mathrm{C}$ & -4.12219400 & 1. 64564800 & 1. 45289500 \\
\hline $\mathrm{C}$ & -4.61079700 & 1. 29820700 & -1.29235300 \\
\hline $\mathrm{C}$ & -5.14631500 & 0.78576500 & 1. 05286000 \\
\hline C & -5.37315000 & 0.63373800 & -0.32705700 \\
\hline $\mathrm{C}$ & 0.46119600 & -3.88082600 & -1.65438000 \\
\hline $\mathrm{C}$ & 1. 82538300 & -4.67776900 & 0.08743400 \\
\hline C & 1. 12287000 & -3.89805800 & 1. 03100700 \\
\hline $\mathrm{C}$ & 0.14119700 & -2.57910300 & -0.92144400 \\
\hline $\mathrm{H}$ & 3. 23368000 & -6.26410700 & -0.22972800 \\
\hline $\mathrm{H}$ & -0.32771100 & -4.62160200 & -1.47994500 \\
\hline C & 2. 69771200 & -5.68661200 & 0.51630400 \\
\hline $\mathrm{C}$ & 1. 28815200 & -4.16933500 & 2. 40286100 \\
\hline $\mathrm{C}$ & 2. 12565400 & -5.18809500 & 2. 82723900 \\
\hline C & 2. 83808300 & -5.93715000 & 1.87558600 \\
\hline $\mathrm{H}$ & 0.74622900 & -3.55063400 & 3.11100000 \\
\hline $\mathrm{H}$ & 2. 24030000 & -5.40030300 & 3. 88572100 \\
\hline $\mathrm{H}$ & 3.50410600 & -6.73122400 & 2. 20296500 \\
\hline 0 & -0.08006900 & -1.90076400 & 1. 38496600 \\
\hline 0 & 1. 71329900 & -4.46543400 & -1.24711300 \\
\hline $\mathrm{C}$ & -1.26369400 & -2.02073800 & -1.30873400 \\
\hline $\mathrm{H}$ & -1.14568600 & -1.46500600 & -2.24350500 \\
\hline $\mathrm{H}$ & -1.54107600 & -1.27682800 & -0.55634500 \\
\hline $\mathrm{C}$ & -2.35939000 & -3.03900400 & -1.47568500 \\
\hline $\mathrm{C}$ & -3.04026700 & -3.81316800 & -0.46027900 \\
\hline $\mathrm{C}$ & -2.90890200 & -3.42069500 & -2.67661200 \\
\hline $\mathrm{C}$ & -3.98464900 & -4.64500500 & -1.12589400 \\
\hline $\mathrm{N}$ & -3.88081600 & -4.38230900 & -2.47643700 \\
\hline
\end{tabular}




\begin{tabular}{|c|c|c|c|}
\hline$C$ & -2.95359800 & -3.88454600 & 0.94304900 \\
\hline C & -3.77933600 & -4.76849000 & 1. 62777100 \\
\hline H & -3.71719700 & -4.82926800 & 2. 71085800 \\
\hline$C$ & -4.70132300 & -5.58641100 & 0.94370200 \\
\hline H & -5.33343200 & -6.26740900 & 1. 50706400 \\
\hline 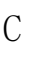 & -4.81905500 & -5.53352000 & -0.44009900 \\
\hline & -2.25936200 & -3.25025700 & 1. 48519800 \\
\hline$t$ & -5.53355300 & -6.15957100 & -0.96871800 \\
\hline & -4.44246400 & -4.80318100 & -3.19967400 \\
\hline & 0.55442900 & -3.71797600 & -2.72998900 \\
\hline & -2.67878700 & -3.07280400 & -3.67496900 \\
\hline & -0.27222300 & -0.19792800 & 1. 17789000 \\
\hline & -2.99184200 & 2. 70011300 & -1.56616900 \\
\hline & -6.17377900 & -0.02166800 & -0.64826700 \\
\hline & -3.90280400 & 1. 78461700 & 2. 50489300 \\
\hline & 3. 97170300 & 1. 48660800 & 1. 59999300 \\
\hline & 5. 87343100 & -2.19069800 & 0.48824200 \\
\hline 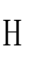 & 4. 36390100 & 0.46384600 & -2.52472300 \\
\hline $\mathrm{C}$ & -4.86912700 & 1. 15262000 & -2.80505200 \\
\hline 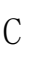 & -5.22719000 & 2. 53592400 & -3.39953500 \\
\hline & -6.12834000 & 2. 94421400 & -2.92740500 \\
\hline & -5.41594100 & 2. 45124300 & -4.47713700 \\
\hline & -4.41924600 & 3. 26132700 & -3.25937100 \\
\hline & -6.02706500 & 0.18542100 & -3.11654300 \\
\hline & -6.97456600 & 0.52758100 & -2.68462600 \\
\hline & -5.82434000 & -0.82457100 & -2.74251600 \\
\hline 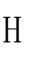 & -6.16648200 & 0.11598800 & -4.20172700 \\
\hline$C$ & -3.59592600 & 0.61197200 & -3.49696700 \\
\hline 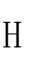 & -3.35122400 & -0.38842300 & -3.12260800 \\
\hline$H$ & -2.72820000 & 1. 25561300 & -3.32161000 \\
\hline H & -3.75065700 & 0.54360900 & -4.58151000 \\
\hline $\mathrm{C}$ & 5. 19902900 & -0.76887000 & 2. 70369300 \\
\hline $\mathrm{C}$ & 5. 70632800 & -1.97659100 & -2.21543800 \\
\hline $\mathrm{C}$ & 6.71829600 & -0.94193700 & 2.93837000 \\
\hline H & 7. 14179800 & -1.73244200 & 2.31003000 \\
\hline H & 6. 91511400 & -1.20693000 & 3. 98476600 \\
\hline H & 7. 25710000 & -0.01367900 & 2. 71582800 \\
\hline $\mathrm{C}$ & 4. 47276100 & -2.09171200 & 3. 03932100 \\
\hline H & 4. 81170200 & -2.91887500 & 2. 40713300 \\
\hline H & 3. 39158600 & -1.98952400 & 2. 89747300 \\
\hline H & 4. 65449900 & -2.37312500 & 4. 08440200 \\
\hline C & 4. 68761600 & 0.31360000 & 3. 67377000 \\
\hline H & 3. 60733000 & 0.46955100 & 3. 57887700 \\
\hline 11 & 5. 18776200 & 1. 27540800 & 3. 51247200 \\
\hline
\end{tabular}




$\begin{array}{lrrr}\mathrm{H} & 4.88852900 & 0.00629300 & 4.70659700 \\ \mathrm{C} & 7.25261600 & -2.00524100 & -2.15124900 \\ \mathrm{H} & 7.65566400 & -2.70382600 & -2.89540500 \\ \mathrm{H} & 7.60995000 & -2.32177100 & -1.16541800 \\ \mathrm{H} & 7.67142300 & -1.01280500 & -2.35450600 \\ \mathrm{C} & 5.29141200 & -1.57267100 & -3.64330600 \\ \mathrm{H} & 5.70844400 & -0.60147700 & -3.93246500 \\ \mathrm{H} & 4.20190500 & -1.52190700 & -3.74989500 \\ \mathrm{H} & 5.66282000 & -2.31507400 & -4.35910100 \\ \mathrm{C} & 5.16015200 & -3.39823000 & -1.94691600 \\ \mathrm{H} & 5.46831700 & -3.77856800 & -0.96736600 \\ \mathrm{H} & 5.53887900 & -4.09645100 & -2.70401700 \\ \mathrm{H} & 4.06588100 & -3.41983700 & -1.98217300 \\ \mathrm{C} & 0.33677200 & -2.74125700 & 0.57748800 \\ \mathrm{C} & -6.05094000 & 0.04861100 & 2.06003400 \\ \mathrm{C} & -6.08023700 & -1.46514300 & 1.74863900 \\ \mathrm{H} & -6.47557100 & -1.67372900 & 0.74933700 \\ \mathrm{H} & -6.72119100 & -1.98680800 & 2.47031900 \\ \mathrm{H} & -5.07897300 & -1.90402900 & 1.80484000 \\ \mathrm{C} & -5.57246700 & 0.22340300 & 3.51435200 \\ \mathrm{H} & -5.59585900 & 1.27183900 & 3.83239900 \\ \mathrm{H} & -4.55285000 & -0.15339600 & 3.65389500 \\ \mathrm{H} & -6.23013500 & -0.33743000 & 4.18831900 \\ \mathrm{C} & -7.48616800 & 0.61830700 & 1.95152000 \\ \mathrm{H} & -8.15678200 & 0.10615700 & 2.65305900 \\ \mathrm{H} & -7.89480500 & 0.49127900 & 0.94324900 \\ \mathrm{H} & -7.50203300 & 1.68918200 & 2.18545700\end{array}$


INT-IV-S (Table S4, entry 16)

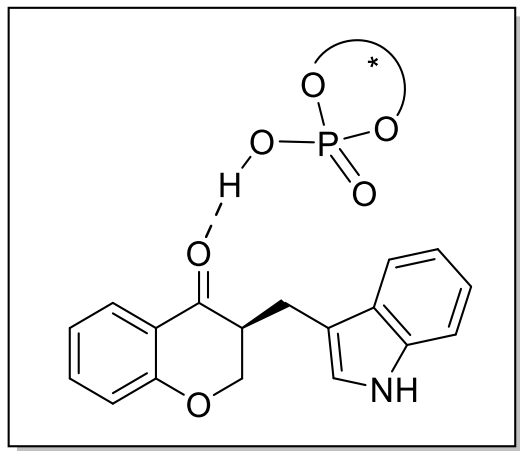

SCF energy [B3LYP-GD3BJ/6-311+G**/SMD (Chlorobenzene)]: -3291.702517840 a.u.

Thermal correction to Gibbs free energy at $258 \mathrm{~K}: 1.111869000$ a.u. Gibbs free energy at $258 \mathrm{~K}$ [B3LYP-GD3BJ/6-311+G**/SMD (Chlorobenzene)]: -3290.590648840 a.u.

01

$\mathrm{P}$

0

0

0

0

C

C

C

C

C

C

C

C

C

C

C

C

C

C

C

C

C

H

H

H

H
$-0.85641800$

$-0.37334600$

$-2.19473900$

$-1.00939200$

0. 10265000

$-0.15406000$

1. 14796700

1. 29459200

0. 20778900

$-1.05928400$

$-2.38145500$

$-3.40102500$

$-2.68568500$

$-1.24166600$

$-2.87474400$

$-4.27828400$

$-4.42006900$

$-5.34014900$

$-5.27023700$

$-4.25392400$

$-3.31072100$

$-3.42913000$

-0. 11950700

2. 29176200

0. 35564500

$-2.48895300$
1. 44762000

2. 95875300

1. 67364200

0. 65857300

0. 89232000

3. 90980900

4. 11447000

5. 05009500

5. 72807900

5. 51355900

6. 10285200

5. 17332700

4. 70100100

4. 63402000

5. 77831600

5. 49452100

4. 00102300

3. 12571500

1. 76947000

1. 24613900

2. 15542300

3. 52588500

-1. 30242900

5. 24117500

6. 41720600

7. 14123500
0. 26567100

0. 64726900

$-0.64024900$

1. 50817600

$-0.86369800$

-0. 35426700

$-0.85720100$

$-1.89736900$

$-2.44505500$

$-1.91498600$

$-2.35301800$

-1. 66222200

$-0.35006900$

$-0.83700700$

0. 76917500

1. 34955500

1. 14820500

1. 71447700

1. 39805500

0. 58014000

0. 06055200

0. 27528800

1. 37401500

-2. 28172000

$-3.27288500$

$-2.00824600$ 


\begin{tabular}{|c|c|c|c|}
\hline H & -2.49504300 & 6. 12005800 & -3.44303000 \\
\hline H & -4.36432400 & 5. 65294600 & -1.46243900 \\
\hline & -3.58897500 & 4. 29792200 & -2.29416200 \\
\hline & -2.75782500 & 6. 79867400 & 0.39030000 \\
\hline & -2.11487200 & 5. 61924300 & 1.54259400 \\
\hline & -5.05618800 & 6. 04742500 & 0.80410200 \\
\hline & -4.36660700 & 5. 78569100 & 2. 40257300 \\
\hline & -6.11238000 & 3. 48934700 & 2. 38793300 \\
\hline & -6.01376800 & 1. 08569800 & 1. 79673800 \\
\hline & -4.23794200 & -0.21011500 & 0.27213400 \\
\hline & -4.49221400 & -1.14536700 & 1. 28525100 \\
\hline & -4.07029800 & -0.66116700 & -1.04528400 \\
\hline & -4.60401800 & -2.51183900 & 1. 00501100 \\
\hline & -4.17366500 & -2.01821700 & -1.36932500 \\
\hline & -4.44454400 & -2.91880300 & -0.32784400 \\
\hline & 2. 35132600 & 3. 40283100 & -0.34517100 \\
\hline & 2. 55846400 & 3. 19519000 & 1. 02176100 \\
\hline & 3. 34634500 & 2. 98897900 & -1.24954500 \\
\hline & 3. 73238100 & 2. 59522500 & 1.50337400 \\
\hline & 4. 53419700 & 2. 40328500 & -0.81256100 \\
\hline & 4. 70460700 & 2. 21879900 & 0.57179000 \\
\hline & 0.52786100 & -3.02994300 & 2. 41942200 \\
\hline & 2. 80300700 & -3.58602500 & 2. 12205800 \\
\hline & 3. 02202000 & -2.40816800 & 1. 37296300 \\
\hline & 1. 88273800 & -1.66790100 & 0.82085000 \\
\hline & 0.49440600 & -2.18435500 & 1. 14775800 \\
\hline & 3. 69738500 & -5.26540800 & 3.11173000 \\
\hline & 0.66918200 & -2.37584000 & 3. 29122300 \\
\hline & 3. 89233300 & -4.36180700 & 2. 54341100 \\
\hline & 4. 34427000 & -2.01335700 & 1. 08702200 \\
\hline$C$ & 5. 41980800 & -2.76978600 & 1.52052700 \\
\hline & 5. 18414100 & -3.95215600 & 2. 24301700 \\
\hline & 4. 48492100 & -1.10684300 & 0.50834000 \\
\hline 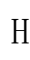 & 6. 43612000 & -2.46137000 & 1. 29521000 \\
\hline & 6. 02360500 & -4.55447100 & 2.58067100 \\
\hline & 2. 04949900 & -0.68899600 & 0.07920500 \\
\hline 0 & 1.56683100 & -4.02519000 & 2. 45133200 \\
\hline C & -0.17481200 & -2.86122300 & -0.09007100 \\
\hline & -0.23667800 & -2.09697500 & -0.87368800 \\
\hline & -1.21067400 & -3.07545300 & 0.19840900 \\
\hline & 0.46437900 & -4.10528700 & -0.64257100 \\
\hline & 1. 52337000 & -4.18990600 & -1.62376400 \\
\hline & 0.12609400 & -5.39993800 & -0.33326900 \\
\hline & 1. 77745000 & -5.57084300 & -1.85351700 \\
\hline
\end{tabular}




\begin{tabular}{|c|c|c|c|}
\hline N & 0.91354100 & -6.28274300 & -1.04678500 \\
\hline $\mathrm{C}$ & 2. 26793500 & -3.23862700 & -2.34518800 \\
\hline $\mathrm{C}$ & 3. 22535200 & -3.67603100 & -3.25160100 \\
\hline $\mathrm{H}$ & 3. 80459700 & -2.94749200 & -3.81245600 \\
\hline $\mathrm{C}$ & 3. 46220700 & -5.05070000 & -3.45777800 \\
\hline $\mathrm{H}$ & 4. 21918600 & -5.36174200 & -4.17273000 \\
\hline $\mathrm{C}$ & 2. 74243700 & -6.01565400 & -2.76320800 \\
\hline $\mathrm{H}$ & 2. 10416400 & -2.17625700 & -2.18911200 \\
\hline $\mathrm{H}$ & 2. 92152400 & -7.07599600 & -2.92339500 \\
\hline $\mathrm{H}$ & 0.84726700 & -7.28732700 & -1.00377500 \\
\hline $\mathrm{H}$ & -0.40366000 & -3.58497900 & 2. 55363300 \\
\hline $\mathrm{H}$ & -0.62602300 & -5.76875400 & 0.35136600 \\
\hline $\mathrm{H}$ & 0.83787700 & 0.31235600 & -0.49776200 \\
\hline $\mathrm{H}$ & -4.54286600 & -3.97417600 & -0.56543500 \\
\hline $\mathrm{H}$ & -4.59093600 & -0.78385400 & 2. 30185000 \\
\hline $\mathrm{H}$ & -3.87776100 & 0.07082100 & -1.81867400 \\
\hline $\mathrm{H}$ & 5. 62476500 & 1. 76655600 & 0.92417600 \\
\hline $\mathrm{H}$ & 3. 16247500 & 3. 12256700 & -2.30859300 \\
\hline $\mathrm{H}$ & 1. 79341500 & 3. 51583300 & 1. 71897300 \\
\hline $\mathrm{C}$ & -4.91477200 & -3.55893200 & 2. 09347000 \\
\hline $\mathrm{C}$ & -6.24772900 & -4.26885500 & 1. 75575800 \\
\hline $\mathrm{H}$ & -6.48528200 & -5.02076500 & 2. 51861700 \\
\hline $\mathrm{H}$ & -6.20523600 & -4.77750700 & 0.78702500 \\
\hline $\mathrm{H}$ & -7.07392000 & -3.54974900 & 1. 71861500 \\
\hline C & -3.77994800 & -4.60862400 & 2. 15012200 \\
\hline H & -3.99904300 & -5.37173100 & 2. 90729400 \\
\hline H & -2.82704900 & -4.13453100 & 2. 41445300 \\
\hline H & -3.64718800 & -5.11891400 & 1. 19018500 \\
\hline C & -5.05034700 & -2.93074800 & 3. 49359000 \\
\hline H & -5.86705200 & -2.20141300 & 3. 53474000 \\
\hline H & -4.12856600 & -2.42822300 & 3. 80734800 \\
\hline H & -5.26965500 & -3.71269300 & 4. 22970100 \\
\hline C & 3. 90278000 & 2. 38605000 & 3. 02070600 \\
\hline C & 5. 25053600 & 1.73232700 & 3. 37984500 \\
\hline H & 5. 35541100 & 0.73925400 & 2. 92798400 \\
\hline H & 5. 32085300 & 1. 60738500 & 4. 46645700 \\
\hline H & 6. 10112000 & 2. 34720000 & 3. 06306700 \\
\hline C & 2. 77106600 & 1. 46713300 & 3. 54013600 \\
\hline H & 2. 83146000 & 0.47832000 & 3. 07201700 \\
\hline H & 1. 77748200 & 1. 87316500 & 3. 32605900 \\
\hline H & 2. 85583800 & 1. 33524800 & 4. 62626100 \\
\hline C & 5. 65057600 & 1. 97353800 & -1.78468800 \\
\hline C & 6. 93333200 & 2. 78339100 & -1.47860800 \\
\hline & 7. 74460700 & 2. 48671000 & -2.15519600 \\
\hline
\end{tabular}


$-0.45193800$

6. 75794900

3. 85749300

$-1.60843300$

C

5. 94664900

0. 46551100

$-1.61204000$

$\mathrm{H}$

6. 29495900

0. 23393400

$-0.59968200$

6. 72989700

0. 14649300

$-2.31099300$

5. 05061900

$-0.13477500$

$-1.80580500$

5. 27318300

2. 21572100

$-3.25878600$

5. 08715400

3. 27604700

-3. 46411800

4. 38110600

1. 64968100

$-3.54967000$

6. 09600700

1. 89435500

$-3.90755300$

$-4.04592100$

$-2.53523100$

$-2.81649000$

$-2.94556100$

$-3.61890700$

$-2.89884300$

$-1.96860000$

$-3.21529300$

$-2.61270100$

$-2.86569500$

$-3.99993600$

$-3.92441600$

-3. 15632600

$-4.47243500$

$-2.24614400$

$-3.68119300$

$-1.41442800$

$-3.80887000$

$-2.73324400$

$-0.93143000$

$-3.54719200$

-4. 45694700

$-0.64192100$

$-3.85675400$

$-3.57411700$

$-1.83535600$

$-4.81509400$

$-5.39746300$

-3. 14840200

$-3.25582400$

$-5.69251100$

$-3.98581300$

$-2.61403800$

$-5.33075800$

$-3.52308400$

$-4.28488400$

-6. 19811000

$-2.40076900$

$-3.21748900$

3. 82739500

3. 75263100

3. 74242600

2. 86878200

4. 25298900

3. 57156500

4. 62176400

4. 42366300

3. 39519000

3. 94453600

3. 61912000

4. 82506200 
(S)-4aa (Table S4, entry 17)

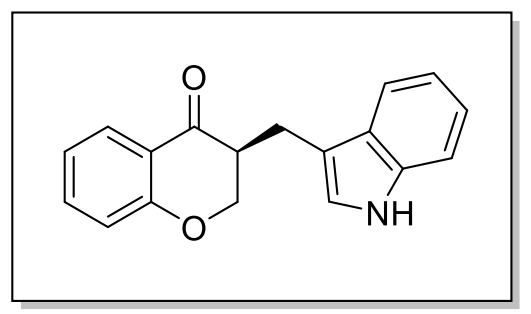

SCF energy [B3LYP-GD3BJ/6-311+G**/SMD (Chlorobenzene)]: -900.502213444 a.u. Thermal correction to Gibbs free energy at $258 \mathrm{~K}: 0.253973000$ a.u.

Gibbs free energy at $258 \mathrm{~K}$ [B3LYP-GD3BJ/6-311+G**/SMD (Chlorobenzene)]: -900.248240444 a.u.

\begin{tabular}{lrrr} 
O 1 & & & \\
$\mathrm{C}$ & -0.57705900 & 0.02037000 & 1.45773500 \\
$\mathrm{C}$ & -2.43231500 & 0.91696800 & 0.29319600 \\
$\mathrm{C}$ & -2.94946600 & -0.37748700 & 0.08051000 \\
$\mathrm{C}$ & -2.08908500 & -1.56805700 & 0.27531300 \\
$\mathrm{C}$ & -0.63569800 & -1.26769400 & 0.64037100 \\
$\mathrm{H}$ & -2.79030900 & 3.02932200 & 0.18061000 \\
$\mathrm{H}$ & -1.11054200 & -0.10898800 & 2.41049600 \\
$\mathrm{C}$ & -3.21346100 & 2.04450300 & 0.01033300 \\
$\mathrm{C}$ & -4.26616200 & -0.51772800 & -0.39150200 \\
$\mathrm{C}$ & -5.04741400 & 0.59617700 & -0.65957900 \\
$\mathrm{C}$ & -4.50978800 & 1.87807000 & -0.46309300 \\
$\mathrm{H}$ & -4.63730400 & -1.52578900 & -0.54945500 \\
$\mathrm{H}$ & -6.06317500 & 0.48068500 & -1.02563000 \\
$\mathrm{H}$ & -5.11437900 & 2.75615800 & -0.67482500 \\
$\mathrm{O}$ & -2.48482700 & -2.71119000 & 0.09589000 \\
O & -1.17138000 & 1.13175200 & 0.76113100 \\
$\mathrm{C}$ & 0.22050000 & -1.22745900 & -0.66127900 \\
$\mathrm{H}$ & -0.12562200 & -0.39865900 & -1.29078600 \\
$\mathrm{H}$ & 0.00544400 & -2.14915600 & -1.21363100 \\
$\mathrm{C}$ & 1.70107200 & -1.10873100 & -0.42975400 \\
$\mathrm{C}$ & 2.48395000 & 0.09959100 & -0.29859600 \\
$\mathrm{C}$ & 2.58555000 & -2.15304300 & -0.30865300 \\
$\mathrm{C}$ & 3.83729000 & -0.29326700 & -0.10052700 \\
$\mathrm{~N}$ & 3.86493600 & -1.67270000 & -0.10471100 \\
$\mathrm{C}$ & 2.17852300 & 1.47310100 & -0.34045500 \\
$\mathrm{C}$ & 3.20440200 & 2.39725000 & -0.18818000 \\
$\mathrm{H}$ & 2.97780700 & 3.45953800 & -0.21823300 \\
$\mathrm{C}$ & 4.53825000 & 1.98281400 & 0.00661900 \\
$\mathrm{H}$ & 5.31868400 & 2.72996400 & 0.12220000 \\
$\mathrm{C}$ & 4.87271100 & 0.63470900 & 0.05373500 \\
& & & \\
\hline
\end{tabular}


1. 80550600

$-0.47763900$

$\mathrm{H}$

5. 90069400

0.31457100

0. 20395500

H

4. $69190800-2.24143500$

$-0.01269100$

H

$-0.27370300$

$-2.09495700$

1. 26121000

$\mathrm{H}$

0.44936500

0. 32524700

1. 66629400

H

2. 40232700

$-3.21812000$

$-0.36222500$ 
INT-I'-nc (Table S5, entry 1)

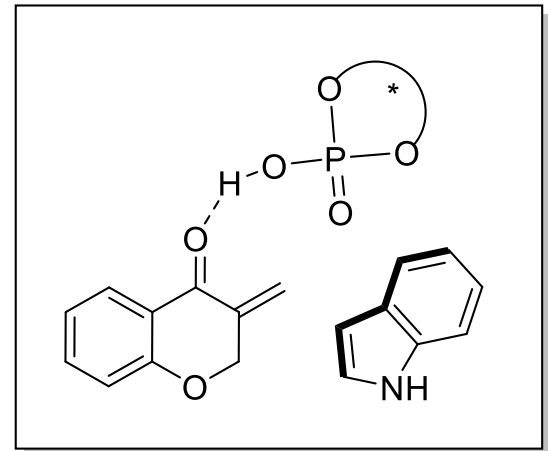

SCF energy [B3LYP-GD3BJ/6-311+G**/SMD (Chlorobenzene)]: -3291.683903880 a.u.

Thermal correction to Gibbs free energy at $258 \mathrm{~K}: 1.109567000$ a.u.

Gibbs free energy at $258 \mathrm{~K}$ [B3LYP-GD3BJ/6-311+G**/SMD (Chlorobenzene)]: -3290.574336880 a.u.

\begin{tabular}{lrrr}
01 & & & \\
$\mathrm{P}$ & -0.43553000 & 1.43988400 & 0.06538700 \\
$\mathrm{O}$ & 0.31966900 & 2.55086500 & 0.98513900 \\
$\mathrm{O}$ & -1.78639100 & 2.17896600 & -0.44703400 \\
$\mathrm{O}$ & -0.93221000 & 0.41764400 & 1.16038100 \\
$\mathrm{O}$ & 0.34181600 & 0.93336000 & -1.08802500 \\
$\mathrm{C}$ & 0.77490800 & 3.71196700 & 0.34058700 \\
$\mathrm{C}$ & 2.09141000 & 3.77432700 & -0.15872500 \\
$\mathrm{C}$ & 2.45075500 & 4.93233600 & -0.87149700 \\
$\mathrm{C}$ & 1.54618800 & 5.96443600 & -1.11493300 \\
$\mathrm{C}$ & 0.26175900 & 5.87789900 & -0.58894000 \\
$\mathrm{C}$ & -0.89403100 & 6.84306500 & -0.73675400 \\
$\mathrm{C}$ & -2.09764700 & 5.99172000 & -0.27708300 \\
$\mathrm{C}$ & -1.50206300 & 4.99933100 & 0.77822800 \\
$\mathrm{C}$ & -0.11726400 & 4.76890500 & 0.18242800 \\
$\mathrm{C}$ & -1.41152400 & 5.70613500 & 2.17288500 \\
$\mathrm{C}$ & -2.82923200 & 5.57583100 & 2.76981800 \\
$\mathrm{C}$ & -3.31911800 & 4.27786200 & 2.16628200 \\
$\mathrm{C}$ & -4.40410200 & 3.49656000 & 2.54725000 \\
$\mathrm{C}$ & -4.66238300 & 2.31205600 & 1.85941000 \\
$\mathrm{C}$ & -3.82251400 & 1.84870000 & 0.83207300 \\
$\mathrm{C}$ & -2.71195300 & 2.64932800 & 0.49650600 \\
$\mathrm{C}$ & -2.49142500 & 3.88309900 & 1.10318300 \\
$\mathrm{H}$ & 3.46659200 & 5.01041700 & -1.24668100 \\
$\mathrm{H}$ & 1.84857800 & 6.82586800 & -1.70527700 \\
$\mathrm{H}$ & -0.76114900 & 7.72605100 & -0.09577100 \\
$\mathrm{H}$ & -1.00518800 & 7.21311100 & -1.76237700 \\
& & & \\
\hline
\end{tabular}




\begin{tabular}{|c|c|c|c|}
\hline H & -2.92299100 & 6.58509700 & 0.12870900 \\
\hline H & -2.48625100 & 5. 41386400 & -1.12334000 \\
\hline & -1.06520100 & 6. 74155000 & 2. 09457100 \\
\hline & -0.69496500 & 5. 16085700 & 2. 79752900 \\
\hline & -3.46785300 & 6. 42009500 & 2. 47365700 \\
\hline & -2.82631200 & 5. 55394100 & 3.86562900 \\
\hline & -5.05059200 & 3. 80423200 & 3.36539400 \\
\hline & -5.53247900 & 1. 71757100 & 2. 12141600 \\
\hline & -4.14649800 & 0.56779600 & 0.14543700 \\
\hline & -4.57220000 & -0.53760000 & 0.89720800 \\
\hline & -4.11837200 & 0.46929600 & -1.25372600 \\
\hline 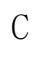 & -4.98793900 & -1.72404000 & 0.28221000 \\
\hline & -4.52403600 & -0.69904700 & $-1.9085960 c$ \\
\hline & -4.95855000 & -1.77415200 & -1.11896700 \\
\hline & 3. 09133300 & 2. 68946100 & 0.03438500 \\
\hline & 3. 24360000 & 2. 05252700 & 1. 27818600 \\
\hline$C$ & 3. 94054600 & 2. 33139100 & -1.01658500 \\
\hline C & 4. 22049900 & 1. 07611500 & 1. 47599800 \\
\hline$C$ & 4. 93934800 & 1. 35840400 & -0.86063800 \\
\hline$C$ & 5. 05707800 & 0.74884500 & 0.39227600 \\
\hline $\mathrm{H}$ & -5.28957700 & -2.68274700 & -1.61351600 \\
\hline & -4.56134100 & -0.45697800 & 1. 97764200 \\
\hline & -3.79091000 & 1. 32877500 & -1.82398900 \\
\hline 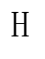 & 5. 82001100 & -0.00662000 & 0.53850400 \\
\hline H & 3. 80182800 & 2. 81109400 & -1.98030700 \\
\hline & 2. 58829400 & 2. 34235800 & 2. 08881600 \\
\hline $\mathrm{C}$ & -5.49051100 & -2.94291800 & 1. 08195500 \\
\hline C & -6.97472700 & -3.20284600 & 0.72908900 \\
\hline & -7.35398800 & -4.07004000 & 1. 28383200 \\
\hline & -7.10887100 & -3.40450600 & -0.33895800 \\
\hline H & -7.59608300 & -2.33696600 & 0.98501900 \\
\hline C & -4.65509100 & -4.19364000 & 0.72038600 \\
\hline & -5.01065400 & -5.06460500 & 1. 28445700 \\
\hline 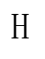 & -3.59631400 & -4.04265200 & 0.95878100 \\
\hline H & -4.72587200 & -4.43913900 & -0.34448100 \\
\hline 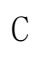 & -5.38999700 & -2.72850900 & 2.60432800 \\
\hline & -6.00354200 & -1.88490700 & 2.94015600 \\
\hline 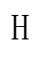 & -4.35729900 & -2.54869200 & 2. 92367400 \\
\hline & -5.74775900 & -3.62308700 & 3. 12667400 \\
\hline $\mathrm{C}$ & 4. 41311000 & 0.36339700 & 2. 82882000 \\
\hline $\mathrm{C}$ & 4. 22559200 & -1.16017300 & 2. 63851700 \\
\hline & 3. 21729000 & -1.38399700 & 2. 27287300 \\
\hline & 4. 36656200 & -1.68521300 & 3. 59173900 \\
\hline & 4. 94456500 & -1.57493900 & 1.92388900 \\
\hline
\end{tabular}




\begin{tabular}{|c|c|c|c|}
\hline C & 3. 40006600 & 0.83547400 & 3. 88949000 \\
\hline $\mathrm{H}$ & 2. 36699400 & 0.64393700 & 3.57852100 \\
\hline $\mathrm{H}$ & 3. 50097700 & 1. 90564400 & 4. 10326200 \\
\hline $\mathrm{H}$ & 3. 57153100 & 0.29528400 & 4. 82794800 \\
\hline $\mathrm{C}$ & 5. 86956900 & 1. 02316700 & -2.04329400 \\
\hline C & 6.73681200 & 2. 26508200 & -2.36207600 \\
\hline $\mathrm{H}$ & 7. 40722500 & 2. 05798500 & -3.20557100 \\
\hline H & 7. 35168100 & 2. 54699900 & -1.49933700 \\
\hline $\mathrm{H}$ & 6. 11939200 & 3. 12975700 & -2.62933900 \\
\hline $\mathrm{C}$ & 6. 80995900 & -0.15810800 & -1.73599700 \\
\hline H & 7. 48190500 & 0.05797300 & -0.89733500 \\
\hline H & 7. 43647800 & -0.36570100 & -2.61097400 \\
\hline $\mathrm{H}$ & 6. 24910800 & -1.07103300 & -1.50637800 \\
\hline C & 5. 03349200 & 0.65117400 & -3.29038500 \\
\hline H & 4. 37989700 & 1. 47011500 & -3.60752500 \\
\hline H & 4. 40519100 & -0.22420000 & -3.09649300 \\
\hline H & 5. 69744900 & 0.41499700 & -4.13104400 \\
\hline $\mathrm{C}$ & -4.54164500 & -0.82244100 & -3.44549900 \\
\hline C & -6.00079600 & -1.01622700 & -3.92349800 \\
\hline H & -6.45147500 & -1.91960100 & -3.49878700 \\
\hline $\mathrm{H}$ & -6.03731100 & -1.10634300 & -5.01628600 \\
\hline H & -6.62419100 & -0.16325400 & -3.63198500 \\
\hline $\mathrm{C}$ & -3.69702900 & -2.04109300 & -3.88663600 \\
\hline $\mathrm{H}$ & -4.07194600 & -2.97717900 & -3.45916700 \\
\hline H & -2.64973500 & -1.92804000 & -3.58328200 \\
\hline H & -3.72126900 & -2.14528900 & -4.97834700 \\
\hline C & -3.97022300 & 0. 42808700 & -4.14065100 \\
\hline $\mathrm{H}$ & -4.56359500 & 1. 32338500 & -3.92382800 \\
\hline H & -3.98016100 & 0.28327800 & -5.22701000 \\
\hline H & -2.93519500 & 0.62507300 & -3.83905900 \\
\hline $\mathrm{C}$ & 5. 83753200 & 0.64403100 & 3. 36306900 \\
\hline $\mathrm{H}$ & 5. 99309700 & 1. 71818000 & 3.51666400 \\
\hline H & 6. 60966700 & 0.29103400 & 2. 67138500 \\
\hline $\mathrm{H}$ & 5. 99320100 & 0.13744200 & 4. 32386900 \\
\hline H & -0.37063200 & -0.40010900 & 1. 31385500 \\
\hline $\mathrm{C}$ & 0.22882200 & -4.74380600 & -0.32171500 \\
\hline $\mathrm{C}$ & 0.77046000 & -5.40130200 & 1. 89061100 \\
\hline $\mathrm{C}$ & 0.88723700 & -4.05261700 & 2. 29990400 \\
\hline $\mathrm{C}$ & 0.32637700 & -2.97678700 & 1. 47054500 \\
\hline C & -0.28552700 & -3.42449700 & 0.19030200 \\
\hline $\mathrm{H}$ & 1. 08063100 & -7.45849600 & 2. 40979200 \\
\hline $\mathrm{H}$ & 1. 25418200 & -4.63282700 & -0.69778900 \\
\hline $\mathrm{C}$ & 1. 18448300 & -6.43230000 & 2. 74658300 \\
\hline $\mathrm{C}$ & 1. 43861000 & -3.76719200 & 3. 56527900 \\
\hline
\end{tabular}




$\begin{array}{lrrr}\mathrm{C} & 1.86233100 & -4.78530600 & 4.40191000 \\ \mathrm{C} & 1.72557900 & -6.12107400 & 3.98619100 \\ \mathrm{H} & 1.50480600 & -2.72646400 & 3.86396200 \\ \mathrm{H} & 2.28825500 & -4.55685700 & 5.37397600 \\ \mathrm{H} & 2.05185600 & -6.92582800 & 4.63978900 \\ \mathrm{O} & 0.33061500 & -1.79840000 & 1.85982700 \\ \mathrm{O} & 0.24507500 & -5.76585000 & 0.69875900 \\ \mathrm{C} & -1.21080100 & -2.69677800 & -0.44768100 \\ \mathrm{H} & -1.67033500 & -3.05113300 & -1.36546600 \\ \mathrm{H} & -1.53980100 & -1.73205000 & -0.07840700 \\ \mathrm{H} & -0.39675800 & -5.13827100 & -1.12351200 \\ \mathrm{C} & 1.99548300 & -3.24145400 & -3.38115300 \\ \mathrm{C} & 2.03388100 & -2.18607600 & -2.42019400 \\ \mathrm{C} & 2.90387700 & -2.20556000 & -1.32135500 \\ \mathrm{C} & 3.74756800 & -3.30412800 & -1.18940500 \\ \mathrm{C} & 3.72804400 & -4.35992500 & -2.12831500 \\ \mathrm{C} & 2.86481400 & -4.33650900 & -3.21741200 \\ \mathrm{C} & 0.99222100 & -2.88246700 & -4.34239300 \\ \mathrm{C} & 0.47868100 & -1.67072300 & -3.94588100 \\ \mathrm{H} & 2.92163000 & -1.38749800 & -0.60722300 \\ \mathrm{H} & 4.43309100 & -3.35171000 & -0.34715000 \\ \mathrm{H} & 4.40380900 & -5.20097700 & -1.99592200 \\ \mathrm{H} & 2.86126900 & -5.15341000 & -3.93532900 \\ \mathrm{H} & 0.69936800 & -3.44313200 & -5.21976500 \\ \mathrm{H} & -0.28465000 & -1.05841800 & -4.40649000 \\ \mathrm{~N} & 1.10101200 & -1.24660600 & -2.79329800 \\ \mathrm{H} & 0.86418400 & -0.40880300 & -2.26064800\end{array}$


INT-I-nc (Table S5, entry 2)

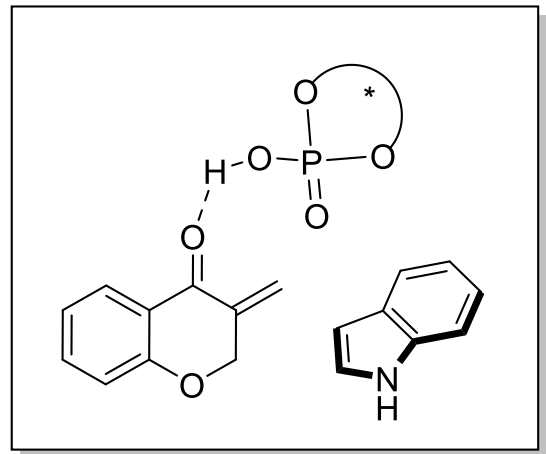

SCF energy [B3LYP-GD3BJ/6-311+G**/SMD (Chlorobenzene)]: -3291.681885020 a.u.

Thermal correction to Gibbs free energy at $258 \mathrm{~K}: 1.106223000$ a.u. Gibbs free energy at $258 \mathrm{~K}$ [B3LYP-GD3BJ/6-311+G**/SMD (Chlorobenzene)]: -3290.575662020 a.u.

\begin{tabular}{lrrr}
01 & & & \\
$\mathrm{P}$ & -0.63060100 & 1.34875800 & -0.09505000 \\
0 & -1.98058800 & 1.78797200 & -0.89404000 \\
0 & 0.00278900 & 2.74837700 & 0.42554300 \\
0 & 0.25992600 & 0.86433400 & -1.30383300 \\
0 & -0.82613000 & 0.41758900 & 1.04214200 \\
$\mathrm{C}$ & -3.00007900 & 2.39989300 & -0.15016600 \\
$\mathrm{C}$ & -4.04334700 & 1.61821400 & 0.38573800 \\
$\mathrm{C}$ & -4.97821500 & 2.27281700 & 1.20708600 \\
$\mathrm{C}$ & -4.87546400 & 3.62981000 & 1.50988400 \\
$\mathrm{C}$ & -3.85390200 & 4.37741500 & 0.93397600 \\
$\mathrm{C}$ & -3.52694700 & 5.84332900 & 1.11794600 \\
$\mathrm{C}$ & -2.09776200 & 5.94159800 & 0.54031900 \\
$\mathrm{C}$ & -2.04017200 & 4.83677300 & -0.56800200 \\
$\mathrm{C}$ & -2.93594800 & 3.77442500 & 0.06070400 \\
$\mathrm{C}$ & -2.65962100 & 5.38792000 & -1.89680900 \\
$\mathrm{C}$ & -1.51083900 & 6.17012000 & -2.57035800 \\
$\mathrm{C}$ & -0.28406600 & 5.42130200 & -2.09637800 \\
$\mathrm{C}$ & 1.01254600 & 5.46493400 & -2.59770300 \\
$\mathrm{C}$ & 1.99466400 & 4.66425800 & -2.01416600 \\
$\mathrm{C}$ & 1.69818500 & 3.77106400 & -0.97203900 \\
$\mathrm{C}$ & 0.36605000 & 3.71954800 & -0.52129000 \\
$\mathrm{C}$ & -0.60844200 & 4.57661700 & -1.02303800 \\
$\mathrm{H}$ & -5.80027400 & 1.69299800 & 1.61585800 \\
$\mathrm{H}$ & -5.59371200 & 4.09403100 & 2.18111200 \\
$\mathrm{H}$ & -4.22820200 & 6.48086900 & 0.56107200 \\
$\mathrm{H}$ & -3.58136600 & 6.15794800 & 2.16638900 \\
& & & \\
\hline
\end{tabular}


$-1.85253800$

6. 93488000

0. 15136400

$-1.36626300$

5. 69806600

1. 31918200

$-3.55471900$

5. 99283500

$-1.72025100$

$-2.94920100$

4. 54010600

$-2.52804900$

$-1.49185300$

7. 21769000

$-2.23779700$

$-1.59656000$

6. 18882500

$-3.66281100$

1. 26418900

6. 11793700

$-3.42973000$

3. 01932000

4. 71726700

$-2.37061000$

2. 77808500

2. 93969000

$-0.37321700$

3. 62965500

2. 19062100

$-1.19480300$

2. 99863400

2. 95775000

1. 01093200

4. 69717300

1. 46096200

$-0.65833700$

4. 06176200

2. 25433800

1. 58649500

4. 89112500

1. 51496500

0. 72953000

$-4.20134300$

0. 16560600

0. 10179600

$-4.04687000$

$-0.33881400$

$-1.19667500$

$-4.58621800$

$-0.71358800$

1. 12275500

$-4.26750100$

$-1.68958800$

$-1.48956000$

$-4.82418600$

$-2.07078600$

0. 87425000

$-4.65619300$

$-2.53517300$

$-0.43897800$

5. 72010900

0. 96172000

1. 16111000

3. 42894800

2. 17294700

$-2.25960700$

2. 33329600

3. 54728300

1. 62935600

$-4.83654300$

$-3.58032300$

$-0.64977700$

$-4.68699300$

$-0.32206500$

2. 13028500

$-3.76109400$

0. 34511400

$-1.98768400$

5. 64965700

0. 62176500

$-1.53242700$

7. 09106000

1. 16638200

$-1.39191700$

7. 78492100

0. 57631200

$-2.00353800$

7. 44270600

1. 12551500

$-0.35574700$

7. 14913200

2. 20980200

$-1.72259400$

5. 61330000

$-0.85460500$

$-1.07135000$

6. 29503900

$-1.46209300$

$-1.67965700$

4. 60469800

$-1.27133700$

$-1.17291700$

5. 91674800

$-0.96222400$

-0. 02461700

5. 26548900

0. 66273900

$-3.02378100$

5. 30798200

1. 67991700

$-3.42953000$

4. 25611200

0. 27074400

$-3.19372500$

5. 96445300

0. 04757400

$-3.60211700$

$-4.09956200$

$-2.18753700$

$-2.93936800$

$-4.35590400$

$-3.70007700$

$-3.07804100$

$-3.66381500$

$-4.28863800$

$-2.46509900$

$-4.21114000$

$-4.00315300$

$-4.12123500$

$-5.37989800$

$-3.97033400$

$-2.79562400$ 
$-2.65810700$

$-1.92617200$

$-1.90045900$

$-2.43977700$

$-2.41148700$

$-0.83510400$

$-2.53382400$

$-2.22441200$

$-5.28365600$

$-2.98802000$

$-6.67056200$

$-2.51400900$

$-7.02004300$

$-3.14794100$

$-7.41325600$

$-2.56176400$

$-6.63765200$

$-1.48109800$

$-5.40861400$

-4. 46178000

-6. 15865900

$-4.59692800$

$-5.71837400$

$-5.07155400$

$-4.45553900$

$-4.86247500$

$-4.27590300$

$-2.91866700$

$-4.18373700$

$-1.90383400$

$-3.27692900$

$-3.24434500$

$-4.60275500$

$-3.56919700$

4. 35767100

2. 29207600

5. 76025700

2. 90493200

6. 54598400

2. 31720400

5. 98911800

5. 81205800

4. 32517300

2. 94396900

3. 92570400

0. 85857500

5. 05364400

0. 20120500

3. 33733300

0. 40284100

4. 55776500

0. 87802600

3. 33243100

3. 14087900

3. 33656300

4. 18763300

3. 57776800

3. 12675600

2. 31452000

2. 75092500

$-5.10264000$

$-1.44473300$

$-4.93830000$

$-0.36227300$

$-6.13527500$

$-1.62926500$

$-4.99950400$

$-1.78803400$

0. 52130400

$-0.09845400$

0. 69028400

$-4.84734600$

1. 85624500

$-5.16563400$

1. 97187300

$-3.77011500$

1. 12365200

$-2.84162100$

0. 24217000

$-3.48390100$

2. 45567600

$-7.11585600$

1. 56462900

$-4.75329100$

2. 56635900

$-6.04919300$

2. 82266600

$-3.28848200$

$-3.42294200$

$-2.81148700$

$-3.37306600$

$-4.46375800$

2. 02585900

2. 52384900

3. 34833200

1. 71921100

2. 88661300

1. 59403700

0. 80668600

2. 45027100

1. 22980200

3. 19719600

3. 59788200

2. 88565000

4. 01771900

3. 09895000

3. 32752200

2. 84094200

4. 39966700

2. 93110300

3. 67770600

3. 19229200

3. 55367200

4. 74961700

3. 87510100

3. 54975000

4. 94315100

3. 76426100

$-3.85444900$

$-3.84399400$

$-3.53630800$

$-4.89134800$

$-1.28400700$

0. 06108500

$-1.97770600$

$-2.17055300$

$-1.40513100$

$-0.39426700$

$-2.63784000$

0. 72002900

$-2.80332900$

-3. 18476100 
$3.53757800-4.16103500$

$-3.98764700$

3. $39764000-5.54602800$

$-3.79471000$

$\mathrm{H}$

$\begin{array}{lll}2.88357300 & -2.21384800 & -3.32268800\end{array}$

$\mathrm{H}$

$\begin{array}{lll}4.19329100 & -3.78127300 & -4.76506300\end{array}$

$\mathrm{H}$

$3.95104400-6.23758100$

$-4.42462600$

0

$1.12586200-1.62735400$

$-1.64924700$

1. 05736600

$-5.71435700$

$-1.03289700$

$-0.84505400$

$-2.86974300$

0. 08792500

$-1.49841700$

$-3.35904500$

0. 80416400

$-1.10614200$

$-1.85622700$

-0. 19154800

$-0.09864500$

$-5.37333300$

0. 60095500

1. 42861700

-3. 26750300

3. 73406200

1. 45914400

$-2.20901800$

2. 77696600

2. 52731600

$-2.04122700$

1. 88573500

3. 57637200

$-2.95226700$

1. 95781600

3. 56613000

$-4.00797100$

2. 89568000

2. 50717100

$-4.17002400$

3. 78106200

0. 19531500

-3. 13257700

4. 45590000

$-0.45540100$

$-2.04352500$

3. 92978500

2. 53861600

$-1.22662100$

1. 16716500

4. 41973600

$-2.84747900$

1. 28041400

4. $40414800-4.69962200$

2. 92686400

2. 51115100

$-4.98390200$

4. 50240700

$-0.15734300$

$-3.75669900$

5. 26585600

$-1.40891200-1.60925200$

4. 19678200

0. 30016500

$-1.47958800$

2. 92474000

$-0.00995200$

$-0.71835400$

2. 32018700 
TS-I'-nc (Table S5, entry 3)

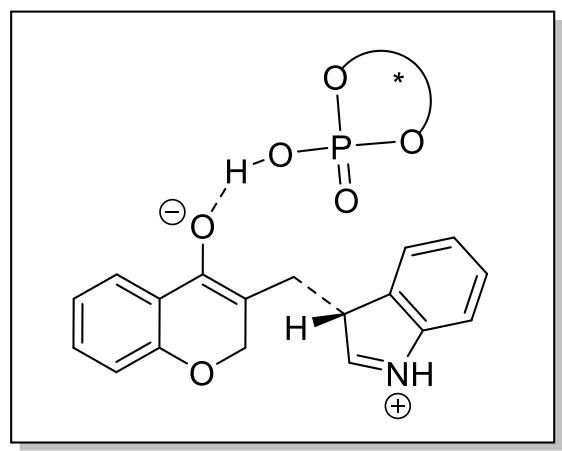

SCF energy [B3LYP-GD3BJ/6-311+G**/SMD (Chlorobenzene)]: -3291.660083890 a.u.

Thermal correction to Gibbs free energy at $258 \mathrm{~K}: 1.113227000$ a.u. Gibbs free energy at $258 \mathrm{~K}$ [B3LYP-GD3BJ/6-311+G**/SMD (Chlorobenzene)]: -3290.546856890 a.u.

01

\begin{tabular}{lrrr}
$\mathrm{P}$ & -0.78567800 & 1.39129700 & -0.28315300 \\
$\mathrm{O}$ & -0.13391500 & 2.38906300 & 0.87928900 \\
$\mathrm{O}$ & -2.27936100 & 2.05554600 & -0.50785500 \\
$\mathrm{O}$ & -0.92477600 & 0.05023700 & 0.41401900 \\
$\mathrm{O}$ & -0.06338700 & 1.50572400 & -1.58689000 \\
$\mathrm{C}$ & 0.09392200 & 3.70105600 & 0.48922200 \\
$\mathrm{C}$ & 1.33797900 & 4.06589600 & -0.06733100 \\
$\mathrm{C}$ & 1.48387700 & 5.37497700 & -0.55396700 \\
$\mathrm{C}$ & 0.43254000 & 6.29097800 & -0.52349100 \\
$\mathrm{C}$ & -0.77662900 & 5.91188300 & 0.05029500 \\
$\mathrm{C}$ & -2.06353100 & 6.70155900 & 0.15715400 \\
$\mathrm{C}$ & -3.09964800 & 5.60412300 & 0.48650900 \\
$\mathrm{C}$ & -2.29648700 & 4.52563500 & 1.28821200 \\
$\mathrm{C}$ & -0.93880600 & 4.63086900 & 0.59935800 \\
$\mathrm{C}$ & -2.20618200 & 4.94842500 & 2.79365300 \\
$\mathrm{C}$ & -3.53683100 & 4.47245400 & 3.41598800 \\
$\mathrm{C}$ & -3.86614300 & 3.25265900 & 2.58293900 \\
$\mathrm{C}$ & -4.77294900 & 2.23247500 & 2.84985800 \\
$\mathrm{C}$ & -4.88064000 & 1.17082100 & 1.95275700 \\
$\mathrm{C}$ & -4.06627400 & 1.07184200 & 0.81097900 \\
$\mathrm{C}$ & -3.15010000 & 2.11959300 & 0.57129900 \\
$\mathrm{C}$ & -3.08336500 & 3.22394300 & 1.41954000 \\
$\mathrm{H}$ & 2.44238900 & 5.66913600 & -0.97272300 \\
$\mathrm{H}$ & 0.56141100 & 7.28482600 & -0.94526100 \\
$\mathrm{H}$ & -2.00670400 & 7.45154800 & 0.95916200 \\
$\mathrm{H}$ & -2.30078300 & 7.24520400 & -0.76469100 \\
$\mathrm{H}$ & -3.96744500 & 5.97487400 & 1.04155300 \\
$\mathrm{H}$ & -3.46442400 & 5.15069700 & -0.44201000 \\
& & & \\
\hline
\end{tabular}




\begin{tabular}{|c|c|c|c|}
\hline$H$ & -2.02630500 & 6. 02147600 & 2. 91681900 \\
\hline H & -1.36975700 & 4. 41362100 & 3. $2579170 \mathrm{c}$ \\
\hline & -4.31752400 & 5. 24167800 & 3. 32666900 \\
\hline & -3.44530800 & 4. 24110900 & 4. 48368600 \\
\hline & -5.39082800 & 2. 25623400 & 3.74434200 \\
\hline & -5.60716600 & 0.38538200 & 2. 13978200 \\
\hline & -4.18028500 & -0.11863400 & -0.07530000 \\
\hline & -4.36033200 & -1.39064000 & 0.48856400 \\
\hline & -4.14766000 & 0.00301200 & -1.47220200 \\
\hline & -4.51104600 & -2.53179100 & $-0.3065540 c$ \\
\hline & -4.27840100 & -1.11395800 & -2.30356200 \\
\hline & -4.46114600 & -2.36607000 & -1.69813800 \\
\hline & 2. 48461200 & 3. 12016500 & -0.14845300 \\
\hline & 2. 88808300 & 2. 38131700 & 0.97726600 \\
\hline & 3. 22354100 & 3. 00606800 & -1.32951100 \\
\hline & 4. 01762000 & 1. 56132700 & 0.94518300 \\
\hline & 4. 36276800 & 2. 18988300 & -1.41308400 \\
\hline & 4. 73966400 & 1. 47901100 & -0.26471500 \\
\hline & -4.57264200 & -3.24102000 & -2.33280200 \\
\hline & -4.35546000 & -1.47673300 & 1. $5684330 \mathrm{c}$ \\
\hline & -4.01041500 & 0.98668500 & -1.89905300 \\
\hline & 5. 64024600 & 0.87480600 & -0.29073300 \\
\hline & 2. 88567000 & 3. 55840100 & -2.20047900 \\
\hline & 2. 30507000 & 2. 47717000 & 1. 88369400 \\
\hline & -4.72519500 & -3.93742200 & 0.29101900 \\
\hline & -6.05858100 & -4.52174400 & -0.23332000 \\
\hline & -6.22706500 & -5.52376300 & 0.18090000 \\
\hline & -6.06696900 & -4.60654300 & -1.32496900 \\
\hline & -6.90357500 & -3.88716900 & 0.05738600 \\
\hline & -3.56098900 & -4.86655000 & -0.12781400 \\
\hline & -3.71822500 & -5.87960000 & 0.26312200 \\
\hline & -2.60724300 & -4.49739800 & 0.26735100 \\
\hline & -3.47237300 & -4.94034600 & -1.21743700 \\
\hline & -4.78677600 & -3.92005600 & 1. 83027500 \\
\hline & -5.61022900 & -3.29677600 & 2. 19677800 \\
\hline & -3.85502400 & -3.55053000 & 2. 27244100 \\
\hline & -4.94966000 & -4.93725300 & 2. 20482700 \\
\hline & 4. 52303600 & 0.80974700 & 2. 19344700 \\
\hline & 4. 71245100 & -0.69218400 & 1. 87814000 \\
\hline & 3. 76207800 & -1.15600400 & 1.59104700 \\
\hline & 5. 08588500 & -1.22326600 & 2. 76159300 \\
\hline & 5. 43671200 & -0.85460800 & 1. 07140000 \\
\hline & 3.53926800 & 0.92265400 & 3. 37416200 \\
\hline & 2. 54773500 & 0.54106600 & 3. 10758700 \\
\hline
\end{tabular}




\begin{tabular}{|c|c|c|c|}
\hline H & 3. 42803300 & 1. 95751500 & 3. 71553800 \\
\hline $\mathrm{H}$ & 3. 91312100 & 0.33689400 & 4. 22201300 \\
\hline $\mathrm{C}$ & 5. 15805800 & 2. 12768400 & -2.73273900 \\
\hline $\mathrm{C}$ & 5. 69544100 & 3. 54052800 & -3.06591300 \\
\hline $\mathrm{H}$ & 6. 26442100 & 3. 52192600 & -4.00362400 \\
\hline $\mathrm{H}$ & 6. 35803400 & 3. 90501200 & -2.27255000 \\
\hline $\mathrm{H}$ & 4. 88320700 & 4. 26510400 & -3.18206900 \\
\hline $\mathrm{C}$ & 6. 36057600 & 1. 16736400 & -2.65619400 \\
\hline $\mathrm{H}$ & 7. 08219000 & 1. 47310500 & -1.89017500 \\
\hline $\mathrm{H}$ & 6. 88556200 & 1. 15767300 & -3.61791800 \\
\hline $\mathrm{H}$ & 6. 05044300 & 0.13712000 & -2.44332700 \\
\hline $\mathrm{C}$ & 4. 23530300 & 1. 65115100 & -3.87964400 \\
\hline $\mathrm{H}$ & 3. 36720300 & 2. 30501700 & -4.00550200 \\
\hline $\mathrm{H}$ & 3. 86188500 & 0.63795100 & -3.69059800 \\
\hline $\mathrm{H}$ & 4. 78477800 & 1. 63596700 & -4.82891000 \\
\hline $\mathrm{C}$ & -4.22706800 & -1.00676100 & -3.84067100 \\
\hline $\mathrm{C}$ & -5.54930500 & -1.53563700 & -4.44470200 \\
\hline $\mathrm{H}$ & -5.73312600 & -2.58152600 & -4.17614200 \\
\hline $\mathrm{H}$ & -5.52346000 & -1.47150200 & -5.53970600 \\
\hline $\mathrm{H}$ & -6.40245100 & -0.94610500 & -4.09010500 \\
\hline $\mathrm{C}$ & -3.04704400 & -1.85383200 & -4.37462100 \\
\hline $\mathrm{H}$ & -3.13911800 & -2.90808100 & -4.09130000 \\
\hline $\mathrm{H}$ & -2.09539300 & -1.47669100 & -3.98057500 \\
\hline $\mathrm{H}$ & -3.00233600 & -1.80454000 & -5.46978900 \\
\hline $\mathrm{C}$ & -4.02308300 & 0.44279100 & -4.32162600 \\
\hline $\mathrm{H}$ & -4.84730100 & 1. 09341400 & -4.00835600 \\
\hline $\mathrm{H}$ & -3.98294600 & 0.46590500 & -5.41701600 \\
\hline $\mathrm{H}$ & -3.08814000 & 0.87093400 & -3.94393900 \\
\hline $\mathrm{C}$ & 5. 88158300 & 1. 41091100 & 2. 62707700 \\
\hline $\mathrm{H}$ & 5.77883100 & 2. 47516400 & 2. 86694100 \\
\hline $\mathrm{H}$ & 6.63658700 & 1. 31832300 & 1. 83860600 \\
\hline $\mathrm{H}$ & 6. 26145400 & 0.89590000 & 3. 51820900 \\
\hline $\mathrm{H}$ & 0.02886400 & -0.76193800 & 1. 19972700 \\
\hline $\mathrm{C}$ & 1. 07848100 & -4.70420700 & 0.40678700 \\
\hline $\mathrm{C}$ & 1. 33122200 & -4.73944700 & 2. 73677900 \\
\hline $\mathrm{C}$ & 1. 37523700 & -3.32865500 & 2. 76140400 \\
\hline $\mathrm{C}$ & 0.82567500 & -2.57993400 & 1. 62150200 \\
\hline $\mathrm{C}$ & 0.54919200 & -3.28460000 & 0.44937400 \\
\hline $\mathrm{H}$ & 1. 62933600 & -6.56598900 & 3. 81414600 \\
\hline $\mathrm{H}$ & 2. 15362400 & -4.73080400 & 0.17381300 \\
\hline $\mathrm{C}$ & 1.68970800 & -5.48364700 & 3. 86451800 \\
\hline $\mathrm{C}$ & 1. 80098000 & -2.68887800 & 3. 93903600 \\
\hline $\mathrm{C}$ & 2. 18753300 & -3.42691800 & 5. 05038400 \\
\hline & 2. 12244900 & -4.82622200 & 5. 01156900 \\
\hline
\end{tabular}


$1.80560700-1.60559100$

3. 96428600

2. $52336500 \quad-2.92036700$

5. 95020500

2. $41442900-5.40808400$

5. 88181500

$0.59631600-1.31049400$

1. 87333600

0

$0.89227100-5.41420800$

1. 64322400

C

$-0.15770000 \quad-2.76521400$

$-0.67317100$

$-0.67662600-3.50949500$

$-1.27312100$

$-0.71766000-1.84154400$

$-0.54869800$

$0.55539600-5.29599900$

$-0.34738200$

2. 10553500

$-3.14327900$

$-2.33318800$

3. $19334600-2.50425900$

$-1.69849500$

4. 42926600

$-3.11860300$

$-1.51585900$

4. 57392700

$-4.41146000$

$-2.02144800$

3. 51703700

$-5.05532200$

$-2.68580500$

2. 27713500

$-4.43414900$

$-2.83947700$

0.96860700

$-2.21881600$

$-2.25185400$

1. 51353300

$-0.99730900$

$-1.76584900$

5. 24764900

$-2.61449000$

$-1.01086700$

5. 52510000

$-4.92304400$

$-1.90763200$

3. 66869100

$-6.05489100$

$-3.08267300$

1. 46391800

$-4.94391500$

$-3.34915700$

0. 15325000

$-2.20677900$

$-2.96609500$

1. 01877100

$-0.02523600$

$-1.66895600$

2. 77165300

$-1.20427600$

$-1.36613400$

3. 34720900

$-0.48532600$

$-0.92951000$ 
TS-I-nc (Table S5, entry 4)

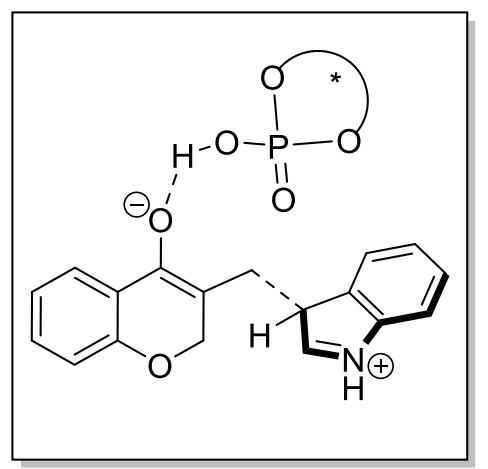

SCF energy [B3LYP-GD3BJ/6-311+G**/SMD (Chlorobenzene)]: -3291.658289830 a.u.

Thermal correction to Gibbs free energy at $258 \mathrm{~K}: 1.111947000$ a.u. Gibbs free energy at $258 \mathrm{~K}$ [B3LYP-GD3BJ/6-311+G**/SMD (Chlorobenzene)]: -3290.546342830 a.u.

\begin{tabular}{lrrr}
01 & & & \\
$\mathrm{P}$ & -0.76808200 & 1.35237500 & 0.15273900 \\
$\mathrm{O}$ & -2.07671000 & 1.60242200 & -0.83922700 \\
$\mathrm{O}$ & -0.32370500 & 2.90991700 & 0.52278700 \\
$\mathrm{O}$ & 0.28406200 & 0.75175000 & -0.76073400 \\
$\mathrm{O}$ & -1.13157800 & 0.68497100 & 1.43561100 \\
$\mathrm{C}$ & -3.19408000 & 2.17480100 & -0.24561200 \\
$\mathrm{C}$ & -4.18909300 & 1.35667100 & 0.32870100 \\
$\mathrm{C}$ & -5.24090000 & 1.98769200 & 1.01366400 \\
$\mathrm{C}$ & -5.30061900 & 3.37371600 & 1.15807900 \\
$\mathrm{C}$ & -4.31902600 & 4.15699300 & 0.56023500 \\
$\mathrm{C}$ & -4.14347700 & 5.65971700 & 0.60677600 \\
$\mathrm{C}$ & -2.68648000 & 5.83886000 & 0.12563800 \\
$\mathrm{C}$ & -2.44170900 & 4.64244900 & -0.85387600 \\
$\mathrm{C}$ & -3.28672800 & 3.56433600 & -0.18168400 \\
$\mathrm{C}$ & -2.99947400 & 4.99887000 & -2.27349900 \\
$\mathrm{C}$ & -1.87341700 & 5.81361200 & -2.94564700 \\
$\mathrm{C}$ & -0.62922100 & 5.22436500 & -2.31771700 \\
$\mathrm{C}$ & 0.69047400 & 5.32217000 & -2.74580900 \\
$\mathrm{C}$ & 1.68317900 & 4.65127700 & -2.03270000 \\
$\mathrm{C}$ & 1.38203100 & 3.84610600 & -0.92177400 \\
$\mathrm{C}$ & 0.03288400 & 3.75102100 & -0.51871000 \\
$\mathrm{C}$ & -0.96027800 & 4.47332700 & -1.17983900 \\
$\mathrm{H}$ & -6.01953100 & 1.36749300 & 1.44903900 \\
$\mathrm{H}$ & -6.10532900 & 3.82931000 & 1.73023000 \\
$\mathrm{H}$ & -4.85101600 & 6.16636900 & -0.06537000 \\
$\mathrm{H}$ & -4.31189200 & 6.07241900 & 1.60836100 \\
& & & \\
\hline
\end{tabular}


-0. 34393200

$-2.00255300$

5. 73968400

0. 97616400

$-3.95505700$

5. 53123200

$-2.22667900$

-3. 16223300

4. 06811200

$-2.82871900$

$-1.96575500$

6. 88557000

$-2.71855800$

$-1.87795600$

5. 72234700

$-4.03808500$

0. 94935100

5. 90892000

$-3.62389800$

2. 72106900

4. 74097700

-2. 34148700

2. 48153900

3. 12432600

$-0.22465000$

3. 43764800

2. 42466300

$-0.97376000$

2. 62010800

3. 17997100

1. 16998200

4. 52673300

1. 79117800

$-0.36421100$

3. 69605700

2. 56614000

1. 82146100

4. 63244100

1. 87630700

1. 03304600

-4. 16515700

$-0.12889400$

0. 23140100

$-3.97653900$

$-0.76981800$

$-1.00547800$

$-4.40629500$

$-0.90888500$

1. 36501300

$-4.04922800$

$-2.15914400$

$-1.12240100$

$-4.48106400$

$-2.30875800$

1. 29461200

$-4.30614400$

$-2.90669400$

0. 04281400

5. 48288900

1. 41039100

1. 52443100

3. 30005200

2. 36975300

$-2.04659300$

1. 87397900

3. 72165300

1. 73608800

$-4.37797300$

$-3.98488500$

$-0.04177700$

$-4.51718500$

$-0.40443900$

2. 31987000

$-3.78376900$

$-0.15443600$

$-1.87490500$

5. 59697600

1. 03111300

$-1.17445300$

6. 98438500

1. 66972200

$-0.92921800$

7. 75707200

1. 14200000

$-1.50212900$

7. 27035000

1. 63555800

0. 12723400

6. 98794000

2. 72011200

$-1.24064000$

5. 63429100

$-0.45109800$

$-0.73143800$

6. 41060800

$-1.00275100$

$-1.27557100$

4. 67462700

$-0.93933200$

$-0.94512600$

5. 85902800

$-0.54759400$

0. 33862800

5. 31585500

1. 05756900

$-2.68898000$

5. 31329800

2. 07964200

$-3.08286400$

4. 35092100

0. 59780600

$-2.93058300$

6. 09582900

0. 50003600

$-3.22023500$

$-3.91036700$

$-2.88443200$

$-2.47607100$

$-2.83279000$

$-3.99013000$

$-2.38679100$

$-1.85253200$

$-3.57113200$

$-2.13456900$

$-2.74046700$

$-4.50757200$

-3. 34959900

$-3.07901800$

$-4.74476700$

$-1.63211300$ 
$-3.51203900$

$-2.56093100$

$-1.92563500$

$-1.42204300$

$-4.27340700$

$-1.15683400$

$-3.39755700$

$-2.48921800$

$-4.74771500$

$-3.12150100$

$-6.08449300$

$-2.66722500$

$-6.28918700$

$-3.23674800$

$-6.91839400$

$-2.82670400$

$-6.06963900$

$-1.60528400$

$-4.84006900$

$-4.63616200$

$-5.65636400$

$-4.87956200$

$-5.03195100$

$-5.16617900$

$-3.90980400$

$-5.03583700$

$-3.60022500$

$-2.87940700$

$-3.49358400$

$-1.81923600$

$-2.64264500$

$-3.22087500$

$-3.78282500$

-3. 43049400

3. 89703300

2. 65851800

5. 22980700

3. 38705300

6. 08887700

2. 85651700

5. 38877700

3. 46804000

5. 22260100

4. 39945300

3. 94623600

1. 24000900

4. 75438500

0. 63502500

3. 00217800

0. 70804300

4. 11496100

1. 29965800

2. 76129000

3. 43458500

2. 70542600

4. 47220400

2. 94071500

3. 45818000

1. 78491100

2. 96636600

$-5.26857500$

$-3.53149400$

$-6.05405100$

$-2.77169100$

$-5.58607800$

$-4.25732200$

$-5.19855100$

$-4.05679600$

0. 89540300

$-0.51635500$

0. 85609500

$-4.53426400$

1. 78704300

$-4.75300000$

2. 07587200

$-3.37144400$

1. 36926200

$-2.47961700$

0. 65467200

$-3.04868200$

2. 07659900

$-6.67288600$

1. 77302000

$-4.74433700$

2. 33050700

$-5.61905300$

2. 93209100

$-2.88649200$
$-3.61488100$

$-3.40928900$

$-3.78740200$

$-4.54813100$

2. 57694600

3. 21023700

4. 12557300

2. 51710700

3. 47556400

2. 31056700

1. 62108700

3. 25068100

1. 89029000

3. 58634100

3. 83623200

3. 17289900

4. 51763400

3. 34811800

3. 64417700

3. 21952100

4. 72667400

3. 22483800

3. 96290200

3. 53681000

3. 79906500

5. 04501800

4. 04289600

3. 69565800

5. 12394000

3. 87700800

$-2.84051900$

$-2.92411700$

$-2.08381400$

$-3.80147500$

$-1.32383100$

$-0.27929300$

$-2.43149200$

$-2.47193200$

$-1.54843600$

$-0.49564500$

$-3.33472600$

0. 29322500

$-3.38442900$

$-3.47612400$ 
$3.49514600-3.75039200 \quad-4.40652900$

3. $18448200-5.11630700$

$-4.36135200$

3. $12894300-1.82081100$

$-3.51640100$

4. $16122100-3.36667900$

$-5.17333900$

3. $61535400-5.79496700$

$-5.09263700$

1. 46103200

$-1.20082200$

$-1.84467100$

0. 93584800

$-5.27433000$

$-1.51010800$

$-0.15997000$

$-2.30166800$

0. 39209400

$-0.89281200$

$-2.86204700$

0. 96709300

$-0.51345900$

$-1.32236000$

0. 09958800

0. 01349100

$-4.96771600$

0. 26342000

1. 44977000

$-2.78236500$

2. 65164700

2. 78950200

$-2.72136500$

2. 20586700

3. $73053700-3.70769900$

2. 49106200

3. 30161900

$-4.78297600$

3. 26945700

1. 97987500

$-4.85767600$

3. 74143400

1. $04598000-3.86843600$

3. 43457100

$0.75771700-1.62480400$

2. 08078500

1. 77222100

$-0.84486500$

1. 47437500

4. 75362200

$-3.64362400$

2. 13195700

4. $00492900-5.57145700$

3. 52051500

$1.68265800-5.70264400$

4. 35576100

0.02564700

$-3.93706200$

3. 80205800

$-0.14251700$

$-1.15038200$

2. 45375900

1. 67329200

0. 09738600

0. 95296700

2. 92525500

$-1.52781200$

1. 48153900

3. 77640300

$-1.19803100$

1. 04179900 
INT-III'-dt (Table S6, entry 1)

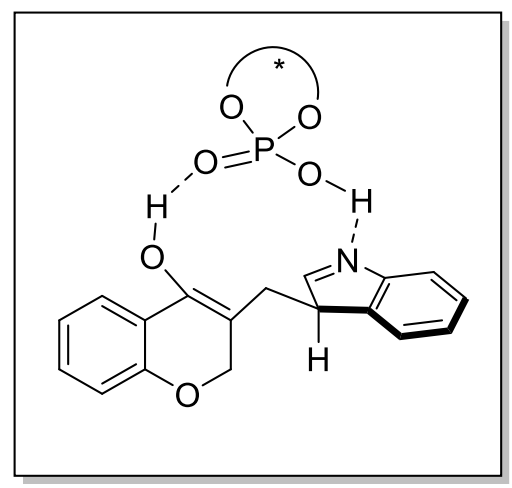

SCF energy [B3LYP-GD3BJ/6-311+G**/SMD (Chlorobenzene)]: -3291.677950730 a.u.

Thermal correction to Gibbs free energy at $258 \mathrm{~K}: 1.112958000$ a.u.

Gibbs free energy at $258 \mathrm{~K}$ [B3LYP-GD3BJ/6-311+G**/SMD (Chlorobenzene)]: -3290.564992730 a.u.

$\begin{array}{crrr}01 & & & \\ \mathrm{H} & -1.51139300 & -1.42542700 & 0.98646700 \\ \mathrm{C} & 1.34331200 & -2.67018700 & -2.04431200 \\ \mathrm{C} & 0.79712000 & -3.96006900 & -2.14140400 \\ \mathrm{C} & 1.62833000 & -5.05245800 & -2.35258500 \\ \mathrm{C} & 3.00876100 & -4.83112500 & -2.45626400 \\ \mathrm{C} & 3.53934000 & -3.54165400 & -2.34310900 \\ \mathrm{C} & 2.70965800 & -2.43426500 & -2.13412500 \\ \mathrm{C} & -0.81226700 & -2.32753800 & -1.81278200 \\ \mathrm{C} & -0.69529400 & -3.82716300 & -1.95420200 \\ \mathrm{H} & 3.67524900 & -5.67286500 & -2.62264000 \\ \mathrm{H} & 4.61339900 & -3.39737500 & -2.41909800 \\ \mathrm{H} & 3.11302300 & -1.43203500 & -2.03301100 \\ \mathrm{H} & 0.43945800 & -0.12783200 & -1.55905600 \\ \mathrm{H} & -1.74876700 & -1.79947000 & -1.66984000 \\ \mathrm{H} & -1.24811200 & -4.14265000 & -2.85266800 \\ \mathrm{~N} & 0.31654400 & -1.70228900 & -1.85552800 \\ \mathrm{C} & 0.35575200 & -5.33878100 & 1.13352600 \\ \mathrm{C} & 0.30580600 & -4.22975500 & 3.23381500 \\ \mathrm{C} & -0.31130500 & -3.09685900 & 2.66118100 \\ \mathrm{C} & -0.92131600 & -3.25876500 & 1.33148300 \\ \mathrm{C} & -0.65951500 & -4.35863300 & 0.59451100 \\ \mathrm{H} & 1.26082400 & -5.08833800 & 4.94470300 \\ \mathrm{H} & 1.37710700 & -5.09152800 & 0.79490600 \\ \mathrm{C} & 0.80913900 & -4.19066100 & 4.53408400 \\ \mathrm{C} & -0.39395100 & -1.92336400 & 3.42427800\end{array}$




\begin{tabular}{|c|c|c|c|}
\hline C & 0.12430300 & -1.87557000 & 4. 71815100 \\
\hline C & 0.71885000 & -3.01139400 & 5. 27330100 \\
\hline H & -0.86290000 & -1.04182900 & 3. 00098700 \\
\hline H & 0.05628500 & -0.95619200 & 5. 2923630 \\
\hline H & 1. 11832100 & -2.98116900 & 6. 2836770 \\
\hline 0 & -1.84506700 & -2.34956700 & 0.8940720 \\
\hline 0 & 0.36414300 & -5.41930000 & 2. 5664410 \\
\hline C & -1.30171700 & -4.61667400 & -0.7459550 \\
\hline H & -1.22610300 & -5.68346200 & -0.9902530 \\
\hline $\mathrm{H}$ & -2.36974700 & -4.37757800 & -0.7090400 \\
\hline H & 0.13839000 & -6.35742300 & 0.7957710 \\
\hline$P$ & -0.23886500 & 1. 27440100 & 0.00113600 \\
\hline 0 & -1.33154300 & 2. 37655400 & -0.5003130 \\
\hline 0 & 0.82572400 & 2. 13494300 & 0.8792630 \\
\hline 0 & 0.45029900 & 0.88929100 & -1.3520330 \\
\hline 0 & -0.78697200 & 0.18559600 & 0.8518870 \\
\hline $\mathrm{C}$ & -2.17264600 & 2. 88786200 & 0.5005490 \\
\hline $\mathrm{C}$ & -3.42070300 & 2. 28104900 & 0.74196200 \\
\hline $\mathrm{C}$ & -4.15848100 & 2. 74577800 & 1. 84487100 \\
\hline $\mathrm{C}$ & -3.67165800 & 3. 73754700 & 2. 69583700 \\
\hline $\mathrm{C}$ & -2.44986600 & 4. 33653500 & 2. 40870400 \\
\hline $\mathrm{C}$ & -1.70176500 & 5. 39985600 & 3. 18399100 \\
\hline $\mathrm{C}$ & -0.28941300 & 5. 33857000 & 2. 55884600 \\
\hline $\mathrm{C}$ & -0.53233500 & 4. 88516400 & 1. 08055400 \\
\hline $\mathrm{C}$ & -1.71816900 & 3. 94934800 & 1. 27601500 \\
\hline $\mathrm{C}$ & -0.94873900 & 6. 12139500 & 0.2124480 \\
\hline C & 0.38363200 & 6. 78878400 & -0.1844230 \\
\hline $\mathrm{C}$ & 1. 33002700 & 5. 61079500 & -0.24128600 \\
\hline $\mathrm{C}$ & 2. 59328700 & 5. 54103400 & -0.8161330 \\
\hline C & 3. 29350100 & 4. 33880600 & -0.76567300 \\
\hline $\mathrm{C}$ & 2. 74387900 & 3. 17062700 & -0.20544500 \\
\hline $\mathrm{C}$ & 1. 44891500 & 3. 27172400 & 0.34737900 \\
\hline $\mathrm{C}$ & 0.76769400 & 4. 48786700 & 0.38573900 \\
\hline H & -5.13014000 & 2. 30153800 & 2. 03896300 \\
\hline H & -4.24754100 & 4. 03794400 & 3. 56770500 \\
\hline $\mathrm{H}$ & -2.15764000 & 6. 39070900 & 3. 04791100 \\
\hline H & -1.69411000 & 5. 20408300 & 4. 26237900 \\
\hline $\mathrm{H}$ & 0.25578800 & 6. 28567200 & 2. 62165400 \\
\hline H & 0.30834000 & 4. 57386700 & 3. 0675920 \\
\hline H & -1.63293600 & 6. 79073900 & 0.74311100 \\
\hline$H$ & -1.46363900 & 5. 76157500 & -0.68560400 \\
\hline$H$ & 0.70503000 & 7. 52137400 & 0.5695200 \\
\hline & 0.32147900 & 7. 32562900 & -1.137960 \\
\hline & 3. 03837700 & 6. 41262000 & -1.289641 \\
\hline
\end{tabular}


H

C

C

C

C

C

C

C

C

C

C

C

C

$\mathrm{H}$

H

H

H

H

$\mathrm{H}$

C

C

H

H

$\mathrm{H}$

C

H

H

$\mathrm{H}$

C

H

$\mathrm{H}$

$\mathrm{H}$

C

C

H

H

$\mathrm{H}$

C

$\mathrm{H}$

H

$\mathrm{H}$

C

C

H
4. 30068900

3. 56213100

4. 38565100

3. 60986500

5. 27470000

4. 46142900

5. 28799600

$-3.97161700$

$-3.92488300$

$-4.60688100$

$-4.50859800$

$-5.20671500$

$-5.14534500$

5. 96081900

4. 32920600

2. 97853000

$-5.61246400$

$-4.60607200$

$-3.43506700$

6. 21403300

7. 13653200

7. 82029000

7. 73876300

6. 56252100

7. 10971600

7. 75045600

6. 52023500

7. 76351300

5. 39077500

4. 74744000

4. 74984300

6. 06012400

$-4.48586500$

$-3.76648200$

$-2.72525900$

$-3.76033500$

$-4.25749700$

$-3.74782400$

$-2.70425900$

$-4.23332400$

$-3.74725000$

$-5.93260500$

$-5.78780000$

$-6.29876600$
4. 29259000

1. 92584800

1. 63635100

1. 07979100

0. 55039100

$-0.02552800$

$-0.26584200$

1. 19397700

1. 27781400

0. 09632000

0. 29405800

$-0.90752100$

$-0.78155300$

$-1.11489500$

2. 27681700

1. 31066700

$-1.54742400$

0. 02875800

2. 13167800

0. 32808000

1. 56207400

1. 42664800

1. 71535200

2. 47754400

$-0.91334500$

$-1.03189400$

$-1.83159400$

$-0.82796900$

0. 15096600

1. 01420000

$-0.73622100$

0. 03079100

0. 36451600

$-0.88354300$

$-0.92159700$

$-0.85895800$

$-1.81229500$

1. 61324500

1. 63850400

2. 53916100

1. 61196300

$-2.10949100$

-2. 13485700

$-3.01672600$
$-1.16722000$

-0. 20170400

$-1.29583300$

0. 92075300

$-1.28808700$

0. 96134400

-0. 15256000

$-0.11101400$

$-1.51068100$

0. 48552300

$-2.31591500$

$-0.28222100$

$-1.67726300$

$-0.12053900$

$-2.17050300$

1. 76774200

$-2.28983800$

1. 56652100

$-1.96004400$

$-2.49154800$

$-2.64455800$

$-3.49183200$

$-1.74178700$

$-2.82240200$

$-2.31891000$

$-3.20000600$

$-2.21376000$

$-1.44372200$

$-3.78904800$

$-3.98749400$

$-3.73548200$

$-4.64991600$

$-3.85594400$

$-4.42053300$

$-4.07904600$

$-5.51728000$

$-4.11089000$

$-4.37546100$

$-4.04261100$

$-4.04713000$

$-5.47151900$

0. 35272600

1. 88666700

2. 28983500 


$\begin{array}{lrrr}\mathrm{H} & -6.23829300 & -1.25321800 & 2.35688600 \\ \mathrm{H} & -4.73594300 & -2.19020900 & 2.18759700 \\ \mathrm{C} & -7.43713800 & -2.03583100 & 0.00097500 \\ \mathrm{H} & -7.88651900 & -1.11488200 & 0.39026900 \\ \mathrm{H} & -7.97467000 & -2.88722700 & 0.43681100 \\ \mathrm{H} & -7.60120000 & -2.05446300 & -1.08197900 \\ \mathrm{C} & -5.33931900 & -3.42868500 & -0.19328300 \\ \mathrm{H} & -4.27445700 & -3.49050700 & 0.05243100 \\ \mathrm{H} & -5.44875800 & -3.51143900 & -1.28018000 \\ \mathrm{H} & -5.84868900 & -4.28992000 & 0.25639100 \\ \mathrm{C} & 4.52949600 & -0.97083800 & 2.17681500 \\ \mathrm{C} & 5.97768200 & -1.02085700 & 2.71905100 \\ \mathrm{H} & 6.68695300 & -1.38963200 & 1.97047800 \\ \mathrm{H} & 6.03443300 & -1.68972300 & 3.58658400 \\ \mathrm{H} & 6.31233000 & -0.02608400 & 3.03548700 \\ \mathrm{C} & 4.09412300 & -2.39105700 & 1.74326600 \\ \mathrm{H} & 4.73584700 & -2.79056200 & 0.95042900 \\ \mathrm{H} & 3.06447900 & -2.38693400 & 1.36943600 \\ \mathrm{H} & 4.14084700 & -3.08080000 & 2.59475300 \\ \mathrm{C} & 3.60742400 & -0.51693200 & 3.32411300 \\ \mathrm{H} & 3.88243200 & 0.47703400 & 3.69630700 \\ \mathrm{H} & 3.68884500 & -1.21932400 & 4.16098700 \\ \mathrm{H} & 2.55535100 & -0.49436600 & 3.02196500 \\ \mathrm{C} & -5.93638400 & 0.40370600 & -4.39329600 \\ \mathrm{H} & -6.47052700 & 1.28110200 & -4.01119300 \\ \mathrm{H} & -6.50580300 & -0.48544500 & -4.10214400 \\ \mathrm{H} & -5.93676800 & 0.45582100 & -5.48913100 \\ \mathrm{H} & 1.22686600 & -6.05952300 & -2.43646900 \\ & & & \\ \mathrm{H} & & \end{array}$


INT-III-dt (Table S6, entry 2)

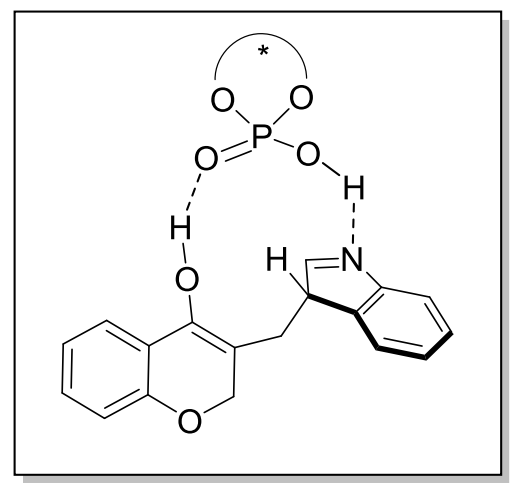

SCF energy [B3LYP-GD3BJ/6-311+G**/SMD (Chlorobenzene)]: -3291.675519570 a.u.

Thermal correction to Gibbs free energy at $258 \mathrm{~K}: 1.112727000$ a.u.

Gibbs free energy at $258 \mathrm{~K}$ [B3LYP-GD3BJ/6-311+G**/SMD (Chlorobenzene)]: -3290.562792570 a.u.

\begin{tabular}{crrr}
01 & & & \\
$\mathrm{H}$ & 1.18859600 & -1.53997000 & -0.78914500 \\
$\mathrm{C}$ & -0.95405700 & -5.02431000 & -2.32494000 \\
$\mathrm{C}$ & 0.27083800 & -3.83440600 & -3.99016100 \\
$\mathrm{C}$ & 0.70749500 & -2.87382200 & -3.05340800 \\
$\mathrm{C}$ & 0.54425600 & -3.20290300 & -1.63061000 \\
$\mathrm{C}$ & -0.23533500 & -4.24030000 & -1.25602000 \\
$\mathrm{H}$ & 0.11835600 & -4.39964300 & -6.04782700 \\
$\mathrm{H}$ & -1.98340600 & -4.65174000 & -2.48521700 \\
$\mathrm{C}$ & 0.45039000 & -3.63055800 & -5.35742900 \\
$\mathrm{C}$ & 1.30240400 & -1.69560400 & -3.52489000 \\
$\mathrm{C}$ & 1.46479900 & -1.47991000 & -4.89327800 \\
$\mathrm{C}$ & 1.04678600 & -2.45128400 & -5.80566100 \\
$\mathrm{H}$ & 1.63205800 & -0.94127000 & -2.81769900 \\
$\mathrm{H}$ & 1.92081600 & -0.55869300 & -5.24391600 \\
$\mathrm{H}$ & 1.17674900 & -2.29037500 & -6.87261400 \\
$\mathrm{O}$ & 1.23339200 & -2.51785900 & -0.67348500 \\
$\mathrm{O}$ & -0.27512800 & -5.02196300 & -3.58863800 \\
$\mathrm{H}$ & -0.73345200 & -5.60795000 & 0.33452700 \\
$\mathrm{H}$ & 0.46275300 & -4.38074000 & 0.76036900 \\
$\mathrm{H}$ & -1.03472800 & -6.08175400 & -2.04966000 \\
$\mathrm{P}$ & 0.21640600 & 1.29713600 & 0.16484500 \\
$\mathrm{O}$ & 1.33294300 & 2.31573200 & 0.76101900 \\
$\mathrm{O}$ & -0.69340100 & 2.23250900 & -0.81054500 \\
$\mathrm{O}$ & -0.59510600 & 1.00279100 & 1.47260100 \\
$\mathrm{O}$ & 0.72092200 & 0.15784300 & -0.64696300 \\
& & & \\
\hline
\end{tabular}




\begin{tabular}{|c|c|c|c|}
\hline $\mathrm{C}$ & 2. 24122200 & 2. 88203500 & -0.14461800 \\
\hline $\mathrm{C}$ & 3. 48262900 & 2. 25381300 & $-0.3709230 c$ \\
\hline $\mathrm{C}$ & 4. 31344000 & 2. 81354300 & -1.35726800 \\
\hline $\mathrm{C}$ & 3. 92398000 & 3. 91962600 & -2.11159400 \\
\hline $\mathrm{C}$ & 2. 70196900 & 4. 52769300 & -1.84409700 \\
\hline $\mathrm{C}$ & 2. 05259800 & 5. 70417500 & -2.53978100 \\
\hline $\mathrm{C}$ & 0.59007700 & 5. 61302400 & -2.05123100 \\
\hline C & 0.68827200 & 4. 98134700 & -0.62139600 \\
\hline $\mathrm{C}$ & 1. 87197500 & 4. 03990500 & -0.82316200 \\
\hline $\mathrm{C}$ & 1. 02210300 & 6. 09786700 & 0.42484700 \\
\hline $\mathrm{C}$ & -0.34034700 & 6. 74666500 & 0.75226100 \\
\hline $\mathrm{C}$ & -1.30074400 & 5. 59112400 & 0.57438500 \\
\hline $\mathrm{C}$ & -2.61486900 & 5. 47513300 & 1.01396600 \\
\hline $\mathrm{C}$ & -3.31107700 & 4. 29200200 & 0.76995300 \\
\hline $\mathrm{C}$ & -2.70591500 & 3. 18965500 & 0.14307200 \\
\hline $\mathrm{C}$ & -1.36507400 & 3. 33327900 & -0.26629700 \\
\hline $\mathrm{C}$ & -0.68013000 & 4. 53553300 & -0.11228700 \\
\hline $\mathrm{H}$ & 5. 28208100 & 2. 35608900 & $-1.5350650 c$ \\
\hline $\mathrm{H}$ & 4. 57400100 & 4. 30048000 & $-2.8955200 c$ \\
\hline $\mathrm{H}$ & 2. 51620400 & 6.65372300 & $-2.2368130 c$ \\
\hline $\mathrm{H}$ & 2. 13795800 & 5. 64698900 & -3.63098200 \\
\hline $\mathrm{H}$ & 0.07175500 & 6. 57710900 & -2.04764700 \\
\hline $\mathrm{H}$ & 0.02519900 & 4. 93260600 & -2.69857200 \\
\hline $\mathrm{H}$ & 1. 76614600 & 6. 80974300 & 0.05382800 \\
\hline $\mathrm{H}$ & 1. 43168300 & 5. 62499300 & 1. 32460800 \\
\hline $\mathrm{H}$ & -0.56954600 & 7. 56715900 & 0.05756900 \\
\hline $\mathrm{H}$ & -0.37542100 & 7. 17066600 & 1. 76229200 \\
\hline $\mathrm{H}$ & -3.09942400 & 6. 29462600 & 1.53898000 \\
\hline $\mathrm{H}$ & -4.34929900 & 4. 20559600 & 1. 07653400 \\
\hline $\mathrm{C}$ & -3.48476000 & 1. 93768700 & -0.06362900 \\
\hline $\mathrm{C}$ & -4.30207400 & 1. 44707700 & 0.95860100 \\
\hline $\mathrm{C}$ & -3.46924900 & 1. 26878900 & -1.30104100 \\
\hline $\mathrm{C}$ & -5.10905300 & 0.31279500 & 0.77815300 \\
\hline $\mathrm{C}$ & -4.25799100 & 0.13920400 & -1.52385900 \\
\hline $\mathrm{C}$ & -5.06803400 & -0.31934900 & -0.46766300 \\
\hline $\mathrm{C}$ & 3. 92312600 & 1. 04498300 & 0.37684400 \\
\hline $\mathrm{C}$ & 3. 74296900 & 0.94699900 & 1.76800600 \\
\hline $\mathrm{C}$ & 4. 56320600 & 0.00088400 & -0.29653600 \\
\hline $\mathrm{C}$ & 4. 17981600 & -0.17166500 & 2. 47701200 \\
\hline $\mathrm{C}$ & 5. 01984000 & -1.14164500 & 0.37886100 \\
\hline $\mathrm{C}$ & 4. 81585000 & -1.20149400 & 1.75979000 \\
\hline $\mathrm{H}$ & -5.68762900 & -1.19237800 & $-0.6352450 c$ \\
\hline $\mathrm{H}$ & -4.29372900 & 1. 95709100 & 1. 91699000 \\
\hline $\mathrm{H}$ & -2.83908400 & 1. 65916700 & $-2.0892820 \mathrm{C}$ \\
\hline
\end{tabular}




\begin{tabular}{|c|c|c|c|}
\hline $\mathrm{H}$ & 5. 15221400 & -2.07607800 & 2. 30390300 \\
\hline $\mathrm{H}$ & 4. 68168000 & 0.07250300 & -1.37338900 \\
\hline & 3. 25508700 & 1. 76368000 & 2. 28272600 \\
\hline & -6.00830300 & -0.17254200 & 1.93229000 \\
\hline & -7.03740200 & 0.93242800 & 2.27190600 \\
\hline & -7.68701400 & 0.61181100 & 3. 09595900 \\
\hline & -7.67174300 & 1. 15689800 & 1. 40661000 \\
\hline & -6.54723900 & 1. 86319300 & 2.57643800 \\
\hline & -6.78478900 & -1.45477600 & 1.57717700 \\
\hline & -7.39880100 & -1.76191100 & 2. 43129500 \\
\hline & -6.11134800 & -2.28620900 & 1. 33885500 \\
\hline & -7.45786100 & -1.30404300 & 0.72553100 \\
\hline & -5.14560400 & -0.46692000 & 3. 18209500 \\
\hline & -4.60763500 & 0.42220500 & 3.52636700 \\
\hline & -4.40293600 & -1.24518100 & 2. 97554700 \\
\hline & -5.77909100 & -0.81242300 & 4. 00846500 \\
\hline & 3. 98737600 & -0.31297900 & 3. 99945400 \\
\hline & 3. 13892500 & -1.57240500 & 4. 29763300 \\
\hline & 2. 14738600 & -1.49676600 & 3. 83702900 \\
\hline & 3. 00366400 & -1.69233500 & 5.38000500 \\
\hline & 3. 61284000 & -2.48391800 & 3.91888600 \\
\hline $\mathrm{C}$ & 3. 26634000 & 0.90175400 & 4. 61480000 \\
\hline $\mathrm{H}$ & 2. 27032500 & 1. 04678100 & 4. 18185000 \\
\hline & 3. 83649900 & 1. 82766900 & 4. 47713300 \\
\hline & 3. 14223500 & 0.74876200 & 5. 69319900 \\
\hline C & 5. 71322200 & -2.26727300 & -0.41292800 \\
\hline C & 4. 78711400 & -2.74441900 & -1.55663500 \\
\hline H & 5. 24593400 & -3.58631800 & -2.08983200 \\
\hline H & 4. 60229500 & -1.95374800 & -2.29137800 \\
\hline & 3. 81493900 & -3.06398500 & -1.16834500 \\
\hline C & 7. 03404100 & -1.72767800 & -1.01160300 \\
\hline H & 6. 85779900 & -0.86985400 & -1.66960400 \\
\hline & 7. 53343100 & -2.50586100 & -1.60225400 \\
\hline $\mathrm{H}$ & 7. 72233900 & -1.40609400 & -0.22102800 \\
\hline $\mathrm{C}$ & 6. 04742600 & -3.48599000 & 0.46815900 \\
\hline $\mathrm{H}$ & 5. 14628600 & -3.92413600 & 0.91196400 \\
\hline 11 & 6. 74102200 & -3.23134900 & 1. 27787400 \\
\hline $\mathrm{H}$ & 6.52649200 & -4.25987600 & -0.14235800 \\
\hline $\mathrm{C}$ & -4.29677100 & -0.58886400 & $-2.8826660 c$ \\
\hline C & -5.72808600 & -0.50255500 & -3.46545300 \\
\hline $\mathrm{H}$ & -6.46726800 & -0.96741700 & -2.80426400 \\
\hline H & -5.77711700 & -1.01428800 & -4.4345450 \\
\hline & -6.02548800 & 0.54136400 & -3.61674400 \\
\hline & -3.91494800 & -2.07634900 & -2.695644 \\
\hline
\end{tabular}




\begin{tabular}{lrrr}
$\mathrm{H}$ & -4.58139300 & -2.58672100 & -1.99188600 \\
$\mathrm{H}$ & -2.88848000 & -2.17212100 & -2.32324700 \\
$\mathrm{H}$ & -3.97378800 & -2.60657300 & -3.65398100 \\
$\mathrm{C}$ & -3.32262000 & 0.02328900 & -3.90750400 \\
$\mathrm{H}$ & -3.56806600 & 1.06807000 & -4.12882500 \\
$\mathrm{H}$ & -3.38076400 & -0.53476400 & -4.84881400 \\
$\mathrm{H}$ & -2.28389000 & -0.01973500 & -3.56187900 \\
$\mathrm{C}$ & 5.36730000 & -0.45160700 & 4.68507300 \\
$\mathrm{H}$ & 5.98707000 & 0.43311100 & 4.49888600 \\
$\mathrm{H}$ & 5.91712900 & -1.32625300 & 4.32196300 \\
$\mathrm{H}$ & 5.24659600 & -0.55999900 & 5.77024500 \\
$\mathrm{C}$ & -1.45067300 & -2.44732800 & 2.85682500 \\
$\mathrm{C}$ & -1.63775200 & -3.75473100 & 2.37220500 \\
$\mathrm{C}$ & -1.79974900 & -4.80705800 & 3.26326200 \\
$\mathrm{C}$ & -1.77450400 & -4.53132700 & 4.63770500 \\
$\mathrm{C}$ & -1.58939500 & -3.22643700 & 5.10624800 \\
$\mathrm{C}$ & -1.42401100 & -2.15920300 & 4.21645600 \\
$\mathrm{C}$ & -1.37810200 & -2.20101800 & 0.68115000 \\
$\mathrm{H}$ & -1.90019600 & -5.34309700 & 5.34876500 \\
$\mathrm{H}$ & -1.57340300 & -3.03809400 & 6.17605400 \\
$\mathrm{H}$ & -1.28022000 & -1.14227200 & 4.56840200 \\
$\mathrm{H}$ & -0.89012700 & 0.02445700 & 1.63072300 \\
$\mathrm{H}$ & -1.29554100 & -1.70524100 & -0.27818600 \\
$\mathrm{H}$ & -2.54695500 & -3.96323300 & 0.40716900 \\
$\mathrm{~N}$ & -1.30493600 & -1.52885600 & 1.77961600 \\
$\mathrm{H}$ & -1.94283500 & -5.82586500 & 2.91189600 \\
$\mathrm{C}$ & -0.45973800 & -4.55406700 & 0.20035600 \\
$\mathrm{C}$ & -1.58449700 & -3.68515800 & 0.86307200 \\
& & & \\
\hline & & &
\end{tabular}


INT-IV'-dt-R (Table S6, entry 3)

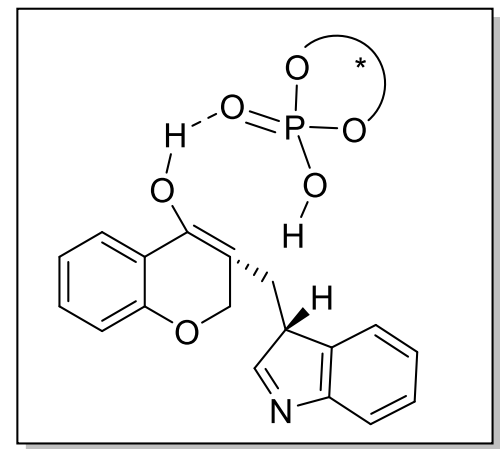

SCF energy [B3LYP-GD3BJ/6-311+G**/SMD (Chlorobenzene)]: -3291.655882490 a.u.

Thermal correction to Gibbs free energy at $258 \mathrm{~K}: 1.113834000$ a.u.

Gibbs free energy at $258 \mathrm{~K}$ [B3LYP-GD3BJ/6-311+G**/SMD (Chlorobenzene)]: -3290.542048490 a.u.

01

P

0

0

0

0

C

C

C

C

C

C

C

C

C

C

C

C

C

C

C

C

C

$\mathrm{H}$

$\mathrm{H}$

$\mathrm{H}$

H
-0. 25742900

0. 78136200

$-1.49642600$

-0. 74030800

0. 26875800

1. 42994000

2. 71832800

3. 23835000

2. 51985800

1. 28449400

0. 33346900

$-0.96617600$

$-0.47944800$

0. 75547200

$-0.07431600$

$-1.39630000$

$-2.19568000$

$-3.33949300$

$-3.91386400$

-3. 34401700

$-2.16234500$

$-1.61921400$

$-0.83897300$

4. 23473700

2. 93650700

0. 70543800
1. 40048700

2. 44447300

2. 29937000

0. 61769600

0. 56267100

3. 32072700

3. 00450600

3. 81557100

4. 88065500

5. 20917000

6. 31994800

5. 92715100

5. 15699800

4. 45931300

6. 17833200

6. 52354700

5. 24994000

4. 84558700

3. 61508900

2. 74207200

3. 16727900

4. 42626700

$-0.27529800$

0. 40561200

$-0.79578600$

1. 03105700

$-1.38196700$

$-0.49421100$

$-0.96844700$

$-1.99307900$

$-2.53387700$

$-1.98721200$

$-2.37835500$

$-1.64062600$

$-0.36917400$

$-0.92525900$

0. 74933700

1. 46654800

1. 30503000

1. 98421400

1. 67162400

0. 72788600

0. 09187400

0. 32619700

$-0.34487000$

0. 85352600

3. 59721300

$-2.36452700$

5. 45756000

-3. 35560900

7. 29801800

$-2.04222400$ 


\begin{tabular}{|c|c|c|c|}
\hline $\mathrm{H}$ & 0.19720200 & 6. 39206300 & -3.46336000 \\
\hline $\mathrm{H}$ & -1.60137100 & 6. 78290100 & -1.39129200 \\
\hline & -1.55460300 & 5. 24394700 & -2.26332400 \\
\hline & 0.44575900 & 7. 05242100 & 0.34554600 \\
\hline & 0.60588000 & 5. 68058700 & 1. 44979700 \\
\hline & -1.90251200 & 7. 37295400 & 0.98623900 \\
\hline & -1.24758700 & 6. 79917500 & 2.51672700 \\
\hline & -3.79080100 & 5. 48169600 & 2. 74150600 \\
\hline 1 & -4.83264900 & 3. 30916800 & 2. 16249700 \\
\hline & -4.02363100 & 1. 45524000 & 0.42245500 \\
\hline & -4.52702900 & 0.66607100 & 1. 46567000 \\
\hline$C$ & -4.26220700 & 1.07144800 & -0.90450500 \\
\hline & -5.27030500 & -0.49116600 & 1. 20934400 \\
\hline & -5.00138000 & -0.07705500 & -1.20485300 \\
\hline & -5.48781000 & -0.83899200 & -0.13184100 \\
\hline $\mathrm{C}$ & 3.57780400 & 1. 93890700 & -0.38368400 \\
\hline C & 3. 70733100 & 1.81873000 & 1. 00366700 \\
\hline $\mathrm{C}$ & 4. 38778100 & 1. 14360500 & -1.21311000 \\
\hline $\mathrm{C}$ & 4. 64365400 & 0.94777800 & 1. 58066500 \\
\hline C & 5. 33975300 & 0.26980000 & -0.68233700 \\
\hline C & 5. 44892400 & 0.19328700 & 0.71936500 \\
\hline $\mathrm{C}$ & -0.30737100 & -3.38381300 & 1. 74286700 \\
\hline $\mathrm{C}$ & -2.10209000 & -4.25136100 & 0.48702900 \\
\hline C & -1.55127700 & -3.75557100 & -0.70891900 \\
\hline $\mathrm{C}$ & 0.13816300 & -2.53233800 & 0.57067300 \\
\hline $\mathrm{H}$ & -3.51639600 & -5.56711900 & 1. 40911900 \\
\hline H & 0.28976300 & -4.30784700 & 1.81293400 \\
\hline C & -3.11069900 & -5.21275900 & 0.46703000 \\
\hline $\mathrm{C}$ & -2.02272900 & -4.25722000 & -1.92859100 \\
\hline $\mathrm{C}$ & -3.01824500 & -5.23318700 & -1.95549500 \\
\hline C & -3.56558600 & -5.70266500 & -0.75821800 \\
\hline H & -1.59719100 & -3.87253600 & -2.84894200 \\
\hline $\mathrm{H}$ & -3.36898700 & -5.62296500 & -2.90669700 \\
\hline $\mathrm{H}$ & -4.34257500 & -6.46240500 & -0.77465700 \\
\hline 0 & -0.22052800 & -2.11775300 & -1.79281400 \\
\hline 0 & -1.69605200 & -3.74607100 & 1. 69257900 \\
\hline $\mathrm{C}$ & 1. 44433700 & -1.78706800 & 0.70980400 \\
\hline $\mathrm{H}$ & 1. 43699500 & -1.13710000 & 1. 59589600 \\
\hline $\mathrm{H}$ & 1.59385800 & -1.12248700 & -0.14323800 \\
\hline $\mathrm{C}$ & 2. 70838800 & -2.69560500 & 0.81108100 \\
\hline $\mathrm{C}$ & 2.84566000 & -3.84348200 & -0.16056600 \\
\hline $\mathrm{C}$ & 2. 92166700 & -3.44619100 & 2. 11351500 \\
\hline $\mathrm{C}$ & 3. 15934700 & -4.98452000 & 0.60313500 \\
\hline & 3. 19842100 & -4.69931900 & 1. 996965 \\
\hline
\end{tabular}


$-1.54240500$

$-2.14723200$

2. 86144600

$-5.27496300$

$-3.22608400$

3. 27723700

$-6.30776800$

$-1.38238600$

3. 44172200

$-7.26177500$

$-1.87626600$

3. 38311700

$-6.22282300$

0. 00956100

2. 45271900

$-3.07718200$

$-2.14545000$

3. 62609600

$-7.08851900$

0. 61815000

$-0.18922200$

$-2.84457800$

2. 68837200

2. 88967000

$-2.96787200$

3. 09207200

0. 11480300

$-1.20216200$

$-1.65392400$

3. 09175900

2. 44574000

1. 63918000

6. 19694900

$-0.46577000$

1. 14325500

4. 26159700

1. 22901200

$-2.28590900$

$-3.88020400$

1. 69867200

$-1.69955600$

$-6.06077500$

$-1.73529500$

-0. 34942400

$-4.32111300$

0. 97013100

2. 48526000

4. 78854100

0. 90002100

3. 11528100

5. 26027500

2. 28466500

3. 62158600

6. 22787400

2. 55280900

3. 18246000

5. 37235300

2. 27414400

4. 71275300

4. 54653300

3. 07471300

3. 36578300

5. 81113200

$-0.15448900$

3. 58024400

6. 82081100

0. 06311600

3. 21400500

5. 53795700

$-1.16337700$

3. 25001500

5. 85600400

-0. 16598400

4. 67513100

3. 42515100

0. 56307000

3. 76357800

3. 07451900

$-0.42630200$

3. 44756200

2. 65005300

1. 28940200

3. 49938900

3. 51547100

0. 55433600

4. 85660300

$-5.30442300$

$-0.50578600$

$-2.65377200$

$-5.85445800$

$-1.36466000$

2. 33679500

$-6.83596200$

$-0.56551200$

$-2.86488900$

$-7.31503300$

$-1.28002000$

$-2.18741200$

$-7.06617600$

$-0.87552300$

$-3.89171000$

$-7.29468600$

0. 41553200

$-2.69479700$

$-4.69290700$

$-1.90110500$

$-2.91336800$

$-5.09186000$

$-2.65846300$

$-2.23117200$

$-3.60580100$

$-1.88236700$

$-2.78352300$

$-4.90971500$

$-2.22705700$

$-3.93863700$

$-4.71848500$

0. 47250600

$-3.69025300$

$-3.62759700$

0. 54173400

$-3.61543700$

$-5.13499200$

1. 48089600

$-3.58319700$

$-4.95841000$

0. 12243600

$-4.70071100$ 


$\begin{array}{lrrr}\text { C } & -7.39254500 & -1.43944800 & 2.18382400 \\ \mathrm{H} & -7.82432700 & -2.05571400 & 2.98210300 \\ \mathrm{H} & -7.68684500 & -1.88267300 & 1.22679800 \\ \mathrm{H} & -7.84353700 & -0.44201400 & 2.24272300 \\ \mathrm{C} & -5.54372600 & -0.80074200 & 3.73678200 \\ \mathrm{H} & -5.96287700 & 0.20253600 & 3.87730700 \\ \mathrm{H} & -4.46576500 & -0.75151100 & 3.92736900 \\ \mathrm{H} & -5.98350900 & -1.45173700 & 4.50082200 \\ \mathrm{C} & -5.26022200 & -2.78913200 & 2.25138900 \\ \mathrm{H} & -5.48235300 & -3.26651400 & 1.29170600 \\ \mathrm{H} & -5.67848900 & -3.42241900 & 3.04419800 \\ \mathrm{H} & -4.17128200 & -2.77147100 & 2.36743500 \\ \mathrm{C} & -0.49983500 & -2.73301200 & -0.61480000 \\ \mathrm{C} & 6.30743600 & -0.53768000 & -1.57136800 \\ \mathrm{C} & 6.32560400 & -2.02691200 & -1.15665100 \\ \mathrm{H} & 6.63864700 & -2.16507500 & -0.11663000 \\ \mathrm{H} & 7.03278000 & -2.58159400 & -1.78502200 \\ \mathrm{H} & 5.33973600 & -2.48882200 & -1.27587900 \\ \mathrm{C} & 5.92720800 & -0.46847400 & -3.06317500 \\ \mathrm{H} & 5.98201900 & 0.55296600 & -3.45597000 \\ \mathrm{H} & 4.91540000 & -0.85008500 & -3.24169800 \\ \mathrm{H} & 6.62200900 & -1.08071100 & -3.64890600 \\ \mathrm{C} & 7.73033100 & 0.05009100 & -1.40391900 \\ \mathrm{H} & 8.44759600 & -0.50082800 & -2.02477300 \\ \mathrm{H} & 8.07066700 & -0.00976500 & -0.36444800 \\ \mathrm{H} & 7.75821200 & 1.10381500 & -1.70456600 \\ \mathrm{H} & 3.56696700 & -2.01152700 & 0.70316400\end{array}$


INT-IV'-dt-S (Table S6, entry 4)

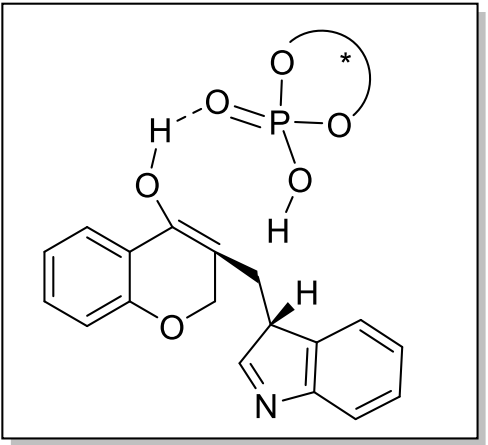

SCF energy [B3LYP-GD3BJ/6-311+G**/SMD (Chlorobenzene)]: -3291.654663860 a.u.

Thermal correction to Gibbs free energy at $258 \mathrm{~K}: 1.112451000$ a.u.

Gibbs free energy at $258 \mathrm{~K}$ [B3LYP-GD3BJ/6-311+G**/SMD (Chlorobenzene)]: -3290.542212860 a.u

$\begin{array}{rrrr}01 & & & \\ 01 & & & \\ P & -0.02274600 & 1.27365400 & 0.01259000 \\ 0 & -1.15267600 & 2.34710800 & -0.43164200 \\ 0 & 1.25014100 & 2.15632700 & 0.49096500 \\ 0 & 0.34562500 & 0.65726300 & -1.41090400 \\ \text { O } & -0.39466600 & 0.29763800 & 1.06958400 \\ \text { C } & -1.62146500 & 3.21481100 & 0.57495300 \\ \text { C } & -2.76281700 & 2.86761500 & 1.31702300 \\ \text { C } & -3.10783100 & 3.69571200 & 2.39756700 \\ \text { C } & -2.35784200 & 4.82437600 & 2.72888400 \\ \text { C } & -1.26785100 & 5.16987000 & 1.93665800 \\ \text { C } & -0.30821100 & 6.32953900 & 2.09080800 \\ \text { C } & 0.87078800 & 5.90924500 & 1.18653600 \\ \text { C } & 0.22002500 & 5.04076900 & 0.05469700 \\ \text { C } & -0.91071400 & 4.38247000 & 0.83216100 \\ \text { C } & -0.31632300 & 5.96681400 & -1.08719300 \\ \text { C } & 0.92033300 & 6.26072400 & -1.96621500 \\ \text { C } & 1.76033100 & 5.01500300 & -1.78325700 \\ \text { C } & 2.85635400 & 4.58223600 & -2.52202600 \\ \text { C } & 3.49915600 & 3.40011800 & -2.15394100 \\ \text { C } & 3.02388700 & 2.59070500 & -1.10832000 \\ \text { C } & 1.86051800 & 3.01766700 & -0.44054700 \\ \text { C } & 1.28227600 & 4.25511200 & -0.70456200 \\ \text { H } & 0.25340900 & -0.32689900 & -1.41317100 \\ \text { H } & -3.98505200 & 3.43547900 & 2.98288100 \\ \text { H } & -2.63150900 & 5.42760300 & 3.59087300\end{array}$




\begin{tabular}{|c|c|c|c|}
\hline $\mathrm{H}$ & -0.76275600 & 7. 26845700 & 1.74423500 \\
\hline $\mathrm{H}$ & -0.00481600 & 6. 49195000 & 3. 13121600 \\
\hline $\mathrm{H}$ & 1. 43758200 & 6. 75634900 & 0.78747300 \\
\hline $\mathrm{H}$ & 1. 56575000 & 5. 28188000 & 1. 75602900 \\
\hline $\mathrm{H}$ & -0.79925200 & 6. 86905400 & -0.69877900 \\
\hline $\mathrm{H}$ & -1.06134300 & 5. 41317900 & -1.66978200 \\
\hline $\mathrm{H}$ & 1. 45618000 & 7. 15456800 & -1.61716200 \\
\hline $\mathrm{H}$ & 0.65885900 & 6. 44115300 & -3.01508800 \\
\hline $\mathrm{H}$ & 3. 22741800 & 5. 16612700 & -3.36057800 \\
\hline $\mathrm{H}$ & 4. 39841100 & 3. 08849400 & -2.67709200 \\
\hline $\mathrm{C}$ & 3. 81415300 & 1. 40078000 & -0.68567100 \\
\hline $\mathrm{C}$ & 4. 30775500 & 0.49004200 & -1.63020000 \\
\hline $\mathrm{C}$ & 4. 20271900 & 1. 27382800 & 0.65606900 \\
\hline $\mathrm{C}$ & 5. 20033400 & -0.52540800 & -1.26172700 \\
\hline $\mathrm{C}$ & 5. 10566700 & 0.28664500 & 1. 06477800 \\
\hline $\mathrm{C}$ & 5. 58659500 & -0.59717400 & 0.08592300 \\
\hline $\mathrm{C}$ & -3.63999200 & 1.71420200 & 0.96579700 \\
\hline $\mathrm{C}$ & -4.32929900 & 1. 71849700 & -0.24805400 \\
\hline $\mathrm{C}$ & -3.86551700 & 0.67875800 & 1. 88488400 \\
\hline $\mathrm{C}$ & -5.26708500 & 0.72158400 & -0.55854500 \\
\hline $\mathrm{C}$ & -4.77013700 & -0.34795100 & 1. 60486000 \\
\hline $\mathrm{C}$ & -5.46380300 & -0.29613000 & 0.38106200 \\
\hline $\mathrm{C}$ & 0.89967000 & -2.94794400 & -2.48910200 \\
\hline $\mathrm{C}$ & -1.24084300 & -3.71358300 & -3.09395100 \\
\hline $\mathrm{C}$ & -1.82573700 & -2.86938600 & -2.12730200 \\
\hline $\mathrm{C}$ & -0.97570400 & -2.37204000 & -1.03562900 \\
\hline $\mathrm{C}$ & 0.37972300 & -2.47438900 & -1.14485100 \\
\hline $\mathrm{H}$ & -1.54201100 & -4.99162100 & -4.78505100 \\
\hline $\mathrm{H}$ & 0.97012300 & -2.12306900 & -3.21887400 \\
\hline $\mathrm{C}$ & -2.02424200 & -4.33802400 & -4.06517000 \\
\hline $\mathrm{C}$ & -3.20970000 & -2.64686100 & -2.17343600 \\
\hline $\mathrm{C}$ & -3.99164800 & -3.25233000 & -3.15430100 \\
\hline $\mathrm{C}$ & -3.39739400 & -4.10207700 & -4.09336100 \\
\hline $\mathrm{H}$ & -3.65607200 & -2.00653600 & -1.42013800 \\
\hline $\mathrm{H}$ & -5.06221700 & -3.07216100 & -3.18384500 \\
\hline $\mathrm{H}$ & -4.00532200 & -4.57929700 & -4.85740100 \\
\hline 0 & -1.66618800 & -1.93064100 & 0.04127400 \\
\hline 0 & 0.09714800 & -3.98473700 & -3.07425500 \\
\hline $\mathrm{C}$ & 1. 37158900 & -2.33412100 & -0.01362200 \\
\hline $\mathrm{H}$ & 0.95424400 & -1.75895100 & 0.81627200 \\
\hline $\mathrm{H}$ & 2. 25452100 & -1.77699200 & -0.35813400 \\
\hline $\mathrm{C}$ & 1. 86509400 & -3.70957900 & 0.53575500 \\
\hline $\mathrm{C}$ & 0.89544100 & -4.47631400 & 1. 40662500 \\
\hline $\mathrm{C}$ & 3. 00034100 & -3.52803800 & 1.52587100 \\
\hline
\end{tabular}




\begin{tabular}{|c|c|c|c|}
\hline C & 1. 50607400 & -4.60649500 & 2. 66948600 \\
\hline $\mathrm{N}$ & 2. 79750600 & -4.00811000 & 2. 70282600 \\
\hline $\mathrm{C}$ & -0.35845600 & -5.02922800 & 1. 17873300 \\
\hline $\mathrm{C}$ & -0.99899100 & -5.69196400 & 2. 23561900 \\
\hline $\mathrm{H}$ & -1.98264900 & -6.12481200 & 2. 07479500 \\
\hline $\mathrm{C}$ & -0.39004300 & -5.80623100 & 3. 48958300 \\
\hline $\mathrm{H}$ & -0.90688000 & -6.32696500 & 4. 29146200 \\
\hline C & 0.87880600 & -5.26505400 & 3. 72161500 \\
\hline $\mathrm{H}$ & -0.83920600 & -4.95973700 & 0.20786400 \\
\hline H & 1. 37069100 & -5.35376200 & 4. 68548300 \\
\hline $\mathrm{H}$ & 1. 89753000 & -3.38316600 & -2.39049700 \\
\hline H & 3. 91913900 & -3.00079700 & 1. 27921500 \\
\hline H & -1.15064600 & -1.27754700 & 0.57100100 \\
\hline $\mathrm{H}$ & 6. 30634200 & -1.35536100 & 0.38047100 \\
\hline $\mathrm{H}$ & 3. 98968900 & 0.59578300 & -2.66118500 \\
\hline H & 3. 81961100 & 1. 99121500 & 1. 37068800 \\
\hline H & -6.18331900 & -1.07778900 & 0.16650500 \\
\hline $\mathrm{H}$ & -3.30620400 & 0.68746700 & 2. 81288200 \\
\hline $\mathrm{H}$ & -4.15104600 & 2. 53421800 & -0.94230500 \\
\hline $\mathrm{C}$ & 5. 80033200 & -1.51288600 & -2.28338700 \\
\hline $\mathrm{C}$ & 7. 33154000 & -1.29722500 & -2.35668000 \\
\hline $\mathrm{H}$ & 7. 78172800 & -1.99143200 & -3.07694700 \\
\hline $\mathrm{H}$ & 7. 81266700 & -1.46289700 & -1.38714100 \\
\hline H & 7. 56715900 & -0.27568600 & -2.67608300 \\
\hline $\mathrm{C}$ & 5.51495600 & -2.96897700 & -1.84711500 \\
\hline $\mathrm{H}$ & 5. 95442100 & -3.67472600 & -2.56232600 \\
\hline $\mathrm{H}$ & 4. 43637700 & -3.16239300 & -1.80428400 \\
\hline $\mathrm{H}$ & 5. 93396900 & -3.19331200 & -0.86087200 \\
\hline $\mathrm{C}$ & 5. 22271800 & -1.31863100 & -3.69853100 \\
\hline $\mathrm{H}$ & 5. 44195700 & -0.32160000 & -4.09641100 \\
\hline $\mathrm{H}$ & 4. 13627500 & -1.46306100 & -3.72045200 \\
\hline $\mathrm{H}$ & 5. 66758500 & -2.05012500 & -4.38237600 \\
\hline $\mathrm{C}$ & -6.06214000 & 0.81302900 & -1.87646100 \\
\hline $\mathrm{C}$ & -7.02085600 & -0.37672000 & -2.07036900 \\
\hline $\mathrm{H}$ & -6.48579500 & -1.33289700 & -2.09378500 \\
\hline H & -7.55174900 & -0.27116700 & -3.02326700 \\
\hline $\mathrm{H}$ & -7.77475900 & -0.42785600 & -1.27665800 \\
\hline $\mathrm{C}$ & -5.08904700 & 0.85051400 & -3.07847900 \\
\hline $\mathrm{H}$ & -4.48534800 & -0.06204600 & -3.12785900 \\
\hline $\mathrm{H}$ & -4.40199500 & 1. 70104000 & -3.02227100 \\
\hline $\mathrm{H}$ & -5.64992100 & 0.93842200 & -4.01737400 \\
\hline $\mathrm{C}$ & -5.03820000 & -1.50880200 & 2.58212800 \\
\hline $\mathrm{C}$ & -6.52764300 & -1.49389900 & 3. 00177400 \\
\hline H & -6.73603800 & -2.31830400 & 3. 69495900 \\
\hline
\end{tabular}


$-6.78563500-0.55439200$

3. 50451300

$-4.71206200-2.85428800$

1. 89068500

$-5.34237400$

$-3.02110200$

1. 01036000

$-4.88483400$

$-3.68712700$

2. 58381700

$-3.66755400$

$-2.88545000$

1. 56428900

$-4.18105500$

$-1.40805000$

3. 85845300

$-4.38862100$

$-0.48956700$

4. 41984700

$-3.10942400$

$-1.43793400$

3. 63325100

$-4.40413500$

$-2.25412800$

4. 51820800

5. 64492700

0. 21408700

2. 50853400

5. 50866900

$-1.21479000$

3. 08158700

4. 46125700

$-1.52571700$

3. 15011500

5. 93377200

$-1.25432000$

4. 09159400

6. 04236500

$-1.95652100$

2. 47769300

4. 90221600

1. 17416000

3. 45837100

3. 82743500

0. 96316600

3. 48969600

5. 03837300

2. 22341300

3. 17299300

5. 29412000

1. 05922300

4. 47517300

7. 14189800

0. 61061900

2. 49041000

7. 73275200

$-0.07180100$

1. 86951300

7. 55646400

0. 58447800

3. 50574500

7. 27546900

1. 62387500

2. 09418700

$-6.90761300$

2. 10982700

$-1.86683900$

$-6.28011100$

3. 00264800

$-1.77687700$

$-7.61352800$

2. 11173500

$-1.02829400$

$-7.48319200$

2. 19781600

$-2.79676000$

2. 21346700

$-4.32120800$

$-0.31064800$ 
INT-IV-dt-R (Table S6, entry 5)

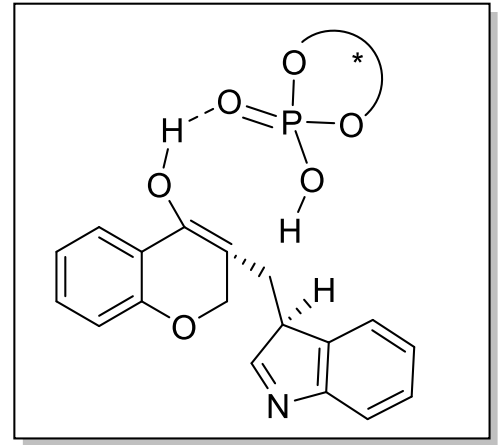

SCF energy [B3LYP-GD3BJ/6-311+G**/SMD (Chlorobenzene)]: -3291.656937380 a.u.

Thermal correction to Gibbs free energy at $258 \mathrm{~K}: 1.112537000$ a.u.

Gibbs free energy at $258 \mathrm{~K}$ [B3LYP-GD3BJ/6-311+G**/SMD (Chlorobenzene)]: -3290.544400380 a.u

$\begin{array}{lrrr}01 & & & \\ \mathrm{P} & -0.61076300 & 1.49238700 & -0.02297700 \\ 0 & 0.22304300 & 2.82042900 & 0.36847300 \\ \mathrm{O} & -2.02642000 & 2.02270800 & -0.62277500 \\ \mathrm{O} & -0.90783000 & 0.96531500 & 1.46175300 \\ \mathrm{O} & 0.02195100 & 0.54881300 & -0.96971100 \\ \mathrm{C} & 0.57529600 & 3.65496800 & -0.71640800 \\ \mathrm{C} & 1.81858000 & 3.48007100 & -1.34839300 \\ \mathrm{C} & 2.06606300 & 4.23673100 & -2.50613000 \\ \mathrm{C} & 1.12083900 & 5.12023000 & -3.02507800 \\ \mathrm{C} & -0.08118800 & 5.30062400 & -2.34936900 \\ \mathrm{C} & -1.25586600 & 6.17695100 & -2.72521600 \\ \mathrm{C} & -2.38755700 & 5.61543400 & -1.83800100 \\ \mathrm{C} & -1.66033000 & 5.08490000 & -0.55659100 \\ \mathrm{C} & -0.34977000 & 4.59522300 & -1.16600100 \\ \mathrm{C} & -1.42684400 & 6.26331400 & 0.44824300 \\ \mathrm{C} & -2.75697200 & 6.38819900 & 1.22415500 \\ \mathrm{C} & -3.26987000 & 4.96422900 & 1.22427000 \\ \mathrm{C} & -4.24618800 & 4.38722300 & 2.03022500 \\ \mathrm{C} & -4.52987200 & 3.02746500 & 1.89097400 \\ \mathrm{C} & -3.82342600 & 2.22248000 & 0.98532000 \\ \mathrm{C} & -2.82741100 & 2.83245700 & 0.20137800 \\ \mathrm{C} & -2.57591800 & 4.19684400 & 0.27499600 \\ \mathrm{H} & -1.68911800 & 0.37593200 & 1.49043400 \\ \mathrm{H} & 3.02328000 & 4.11238400 & -3.00369200 \\ \mathrm{H} & 1.32778000 & 5.66277900 & -3.94412300 \\ \mathrm{H} & -1.05446900 & 7.23293700 & -2.49590300\end{array}$




\begin{tabular}{|c|c|c|c|}
\hline $\mathrm{H}$ & -1.49346500 & 6. 12684600 & -3.79378000 \\
\hline $\mathrm{H}$ & -3.16333600 & 6. 35068700 & -1.60173200 \\
\hline $\mathrm{H}$ & -2.86972300 & 4. 77175600 & -2.34474800 \\
\hline H & -1.12823500 & 7. 18722600 & -0.05656500 \\
\hline H & -0.62228200 & 5. 98458700 & 1. 13804500 \\
\hline H & -3.45775700 & 7. 06174700 & 0.71118100 \\
\hline H & -2.62060800 & 6. 78657500 & 2. 23576200 \\
\hline H & -4.78099000 & 4. 98280200 & 2. 76564600 \\
\hline H & -5.30256600 & 2. 56726100 & 2. 50026800 \\
\hline $\mathrm{C}$ & -4.10461900 & 0.76294400 & 0.87401200 \\
\hline C & -3.99727300 & -0.07302200 & 1. 99177100 \\
\hline C & -4.50048200 & 0.21588900 & -0.35800400 \\
\hline C & -4.26591800 & -1.45290300 & 1. 90018000 \\
\hline $\mathrm{C}$ & -4.80467100 & -1.13894800 & -0.48360100 \\
\hline C & -4.66836100 & -1.95155000 & 0.65891100 \\
\hline C & 2. 87502500 & 2.56790800 & -0.83099200 \\
\hline C & 3. 36467400 & 2. 71878700 & 0.47165000 \\
\hline C & 3. 46106700 & 1. 61936400 & -1.67556600 \\
\hline $\mathrm{C}$ & 4. 43999000 & 1. 95348400 & 0.93796300 \\
\hline $\mathrm{C}$ & 4. 53294200 & 0.82592100 & -1.24799400 \\
\hline C & 5. 00273900 & 1. 01327700 & 0.06089400 \\
\hline C & -0.31994800 & -4.34404500 & 0.93039900 \\
\hline $\mathrm{C}$ & -1.36399100 & -5.04232400 & -1.07547900 \\
\hline C & -0.61044300 & -4.12090800 & -1.82695900 \\
\hline C & 0.24847700 & -3.12813200 & 0.22994300 \\
\hline H & -2.65684200 & -6.74851900 & -1.08519300 \\
\hline H & 0.43859300 & -5.14034400 & 1. 04476500 \\
\hline $\mathrm{C}$ & -2.08092800 & -6.06214200 & -1.69835200 \\
\hline C & -0.59289600 & -4.24766000 & -3.22166900 \\
\hline C & -1.29529300 & -5.27534200 & -3.85122900 \\
\hline C & -2.04161700 & -6.17774500 & -3.08914900 \\
\hline H & -0.02754800 & -3.52450600 & -3.79946900 \\
\hline H & -1.26623200 & -5.36728600 & -4.93326000 \\
\hline H & -2.59263100 & -6.97865900 & -3.57507600 \\
\hline 0 & 0.51960700 & -2.03097900 & -1.91084300 \\
\hline 0 & -1.46621800 & -4.91209300 & 0.28458200 \\
\hline C & 1.04845300 & -2.15950000 & 1. 06294400 \\
\hline H & 0.38940300 & -1.58230200 & 1. 72989800 \\
\hline H & 1. 55241300 & -1.42933600 & 0.42346200 \\
\hline C & 2. 10817200 & -2.85948500 & 1. 96265800 \\
\hline C & 3. 28885700 & -3.52269400 & 1. 28921200 \\
\hline C & 2. 84833700 & -1.91027900 & 2. 88614400 \\
\hline C & 4. 44625800 & -2.97514500 & 1. 87566600 \\
\hline & 4. 13332500 & -1.98205800 & 2. 84448700 \\
\hline
\end{tabular}




\begin{tabular}{|c|c|c|c|}
\hline $\mathrm{C}$ & 3. 40157300 & -4.50466900 & 0.31209800 \\
\hline $\mathrm{C}$ & 4. 68358700 & -4.93256600 & -0.06260900 \\
\hline $\mathrm{H}$ & 4. 78822900 & -5.69582500 & -0.82886700 \\
\hline C & 5. 82676200 & -4.39251800 & 0.53520800 \\
\hline H & 6. 80866800 & -4.74603300 & 0.23213500 \\
\hline C & 5. 72132900 & -3.40102800 & 1. 51704700 \\
\hline H & 2. 52417200 & -4.93092200 & -0.16469700 \\
\hline H & 6. 59875300 & -2.97370400 & 1. 99308400 \\
\hline $\mathrm{H}$ & -0.66691500 & -4.08797600 & 1. 93770800 \\
\hline H & 2. 34368700 & -1.21319700 & 3. 55317700 \\
\hline H & 0.47588600 & -1.16030300 & -1.46072200 \\
\hline H & 2. 91230200 & 3. 47025600 & 1. 11139500 \\
\hline $\mathrm{H}$ & 5. 83099000 & 0.41166400 & 0.40843400 \\
\hline $\mathrm{H}$ & 3. 05584800 & 1. 49870600 & -2.67555700 \\
\hline H & -4.57618400 & 0.87955400 & -1.21013300 \\
\hline H & -4.88276100 & -3.00869700 & 0.56346400 \\
\hline H & -3.71229300 & 0.36808600 & 2. 94388400 \\
\hline $\mathrm{C}$ & 4. 99557600 & 2. 20680000 & 2. 35449200 \\
\hline C & 5.57870300 & 3. 63961700 & 2. 41224700 \\
\hline H & 6. 39549800 & 3. 75929700 & 1. 69093200 \\
\hline $\mathrm{H}$ & 5. 97599700 & 3. 85050600 & 3. 41314900 \\
\hline H & 4. 82029100 & 4. 39701900 & 2. 18581100 \\
\hline $\mathrm{C}$ & 6. 10832600 & 1. 21403100 & 2. 74264300 \\
\hline $\mathrm{H}$ & 6.97051200 & 1. 28222300 & 2. 06890500 \\
\hline H & 5. 74412200 & 0.18106200 & 2. 75006100 \\
\hline H & 6. 46655100 & 1. 44645700 & 3. 75255400 \\
\hline C & 3. 86150000 & 2. 07722100 & 3. 39799700 \\
\hline $\mathrm{H}$ & 3. 44211100 & 1. 06597700 & 3. 38715900 \\
\hline H & 3. 04574300 & 2. 78411800 & 3.21414000 \\
\hline H & 4. 25048300 & 2. 27235100 & 4. 40500600 \\
\hline $\mathrm{C}$ & -5.28793300 & -1.75914600 & -1.80793700 \\
\hline C & -4.13475400 & -2.34433600 & 3. 15012500 \\
\hline $\mathrm{C}$ & -6.69963900 & -2.35902300 & -1.60099100 \\
\hline H & -6.70278100 & -3.13863200 & -0.83191100 \\
\hline H & -7.05895000 & -2.81047500 & -2.53364800 \\
\hline H & -7.41668100 & -1.58674800 & -1.29872900 \\
\hline C & -4.31394300 & -2.87656000 & -2.24508700 \\
\hline H & -4.23835300 & -3.67477100 & -1.50061500 \\
\hline H & -3.30600800 & -2.48224200 & -2.41054300 \\
\hline H & -4.65672300 & -3.33077100 & -3.18238100 \\
\hline $\mathrm{C}$ & -5.36852300 & -0.72279600 & -2.94517000 \\
\hline H & -4.39136700 & -0.27529600 & -3.15822800 \\
\hline H & -6.07532200 & 0.08398100 & -2.71829900 \\
\hline H & -5.71317900 & -1.21342900 & -3.86227900 \\
\hline
\end{tabular}




$\begin{array}{lrrr}\mathrm{C} & -5.22169800 & -1.93883200 & 4.17447400 \\ \mathrm{H} & -5.14360800 & -2.55460300 & 5.07883000 \\ \mathrm{H} & -6.22500900 & -2.07596500 & 3.75549900 \\ \mathrm{H} & -5.12469500 & -0.88950500 & 4.47438400 \\ \mathrm{C} & -2.73986300 & -2.15286200 & 3.79086200 \\ \mathrm{H} & -2.56776600 & -1.12075700 & 4.11381900 \\ \mathrm{H} & -1.94557500 & -2.42265200 & 3.08500100 \\ \mathrm{H} & -2.63838500 & -2.79365700 & 4.67477300 \\ \mathrm{C} & -4.30389000 & -3.84066400 & 2.82152500 \\ \mathrm{H} & -5.30839200 & -4.06395900 & 2.44466900 \\ \mathrm{H} & -4.16018600 & -4.43430800 & 3.73151300 \\ \mathrm{H} & -3.57441700 & -4.18240000 & 2.07819700 \\ \mathrm{C} & 0.08015400 & -3.03824800 & -1.10892200 \\ \mathrm{C} & 5.14401300 & -0.20938500 & -2.21267100 \\ \mathrm{C} & 6.40100800 & -0.88454000 & -1.63241600 \\ \mathrm{H} & 7.18416100 & -0.15316800 & -1.39919600 \\ \mathrm{H} & 6.81153100 & -1.58711700 & -2.36692000 \\ \mathrm{H} & 6.17853400 & -1.45533700 & -0.72515200 \\ \mathrm{C} & 4.09522400 & -1.30670000 & -2.51247000 \\ \mathrm{H} & 3.17565300 & -0.89236000 & -2.93784100 \\ \mathrm{H} & 3.82084800 & -1.84836500 & -1.60114600 \\ \mathrm{H} & 4.49927000 & -2.03458400 & -3.22762000 \\ \mathrm{C} & 5.55012100 & 0.48503100 & -3.53491700 \\ \mathrm{H} & 5.99809500 & -0.24369500 & -4.22150900 \\ \mathrm{H} & 6.28553400 & 1.27791100 & -3.35420600 \\ \mathrm{H} & 4.69225700 & 0.93338200 & -4.04645500 \\ \mathrm{H} & 1.58477200 & -3.59014000 & 2.60094700 \\ & & & \\ & & & \\ \mathrm{H} & & & \end{array}$


INT-IV-dt-S (Table S6, entry 6)

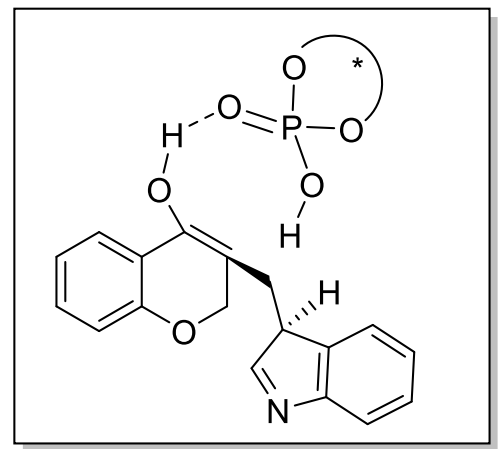

SCF energy [B3LYP-GD3BJ/6-311+G**/SMD (Chlorobenzene)]: -3291.656292930 a.u.

Thermal correction to Gibbs free energy at $258 \mathrm{~K}: 1.111386000$ a.u. Gibbs free energy at $258 \mathrm{~K}$ [B3LYP-GD3BJ/6-311+G**/SMD (Chlorobenzene)]: -3290.544906930 a.u.

01

\begin{tabular}{lrrr}
$\mathrm{P}$ & 0.09198100 & 1.41441300 & -0.16000500 \\
$\mathrm{O}$ & 1.24758800 & 2.38224700 & 0.43366100 \\
$\mathrm{O}$ & -1.09340000 & 2.40621500 & -0.64839700 \\
$\mathrm{O}$ & -0.40507200 & 0.70916500 & 1.17931400 \\
$\mathrm{O}$ & 0.48177200 & 0.50618000 & -1.27075500 \\
$\mathrm{C}$ & 1.87289200 & 3.22200900 & -0.51288200 \\
$\mathrm{C}$ & 3.04839100 & 2.79220700 & -1.15213400 \\
$\mathrm{C}$ & 3.54369000 & 3.58970500 & -2.19684700 \\
$\mathrm{C}$ & 2.90303700 & 4.76347800 & -2.59471300 \\
$\mathrm{C}$ & 1.77486200 & 5.18831500 & -1.90072000 \\
$\mathrm{C}$ & 0.91144400 & 6.40779400 & -2.14020100 \\
$\mathrm{C}$ & -0.36172600 & 6.08162800 & -1.32793600 \\
$\mathrm{C}$ & 0.13957900 & 5.18690000 & -0.14371700 \\
$\mathrm{C}$ & 1.27257100 & 4.43623000 & -0.82826900 \\
$\mathrm{C}$ & 0.67438000 & 6.09146400 & 1.01741000 \\
$\mathrm{C}$ & -0.58502400 & 6.48779100 & 1.81821200 \\
$\mathrm{C}$ & -1.49674500 & 5.29931600 & 1.60572000 \\
$\mathrm{C}$ & -2.65202500 & 4.95196300 & 2.29685200 \\
$\mathrm{C}$ & -3.34649800 & 3.80284000 & 1.92256500 \\
$\mathrm{C}$ & -2.87870400 & 2.94377600 & 0.91260100 \\
$\mathrm{C}$ & -1.67172800 & 3.29554100 & 0.27729400 \\
$\mathrm{C}$ & -1.02178400 & 4.49378200 & 0.55926100 \\
$\mathrm{H}$ & -0.43691200 & -0.27344100 & 1.07232500 \\
$\mathrm{H}$ & 4.44963400 & 3.26730900 & -2.70180000 \\
$\mathrm{H}$ & 3.29070800 & 5.33975400 & -3.43103700 \\
$\mathrm{H}$ & 1.40001500 & 7.31920800 & -1.76776800 \\
& & & \\
\hline
\end{tabular}




\begin{tabular}{|c|c|c|c|}
\hline $\mathrm{H}$ & 0.70241500 & 6. 57481000 & -3.20286800 \\
\hline $\mathrm{H}$ & -0.89664500 & 6. 97075000 & -0.97967500 \\
\hline $\mathrm{H}$ & -1.05321400 & 5. 49610600 & -1.9443890 \\
\hline $\mathrm{H}$ & 1. 24350600 & 6. 94972100 & 0.64670300 \\
\hline $\mathrm{H}$ & 1. 34241000 & 5. 49561600 & 1. 64952800 \\
\hline $\mathrm{H}$ & -1.03660500 & 7. 40920100 & 1. 424261 \\
\hline $\mathrm{H}$ & -0.37187900 & 6. 67047300 & 2. 877539 \\
\hline $\mathrm{H}$ & -3.02372300 & 5. 57397600 & 3. 10720100 \\
\hline $\mathrm{H}$ & -4.28172500 & 3. 55518700 & 2.4154020 \\
\hline $\mathrm{C}$ & -3.70543700 & 1. 76899500 & 0.5203760 \\
\hline $\mathrm{C}$ & -4.30711100 & 0.96895900 & 1. 50259100 \\
\hline $\mathrm{C}$ & -4.00838600 & 1. 53666300 & -0.8296390 \\
\hline $\mathrm{C}$ & -5.22168200 & -0.03651800 & 1. 16570400 \\
\hline $\mathrm{C}$ & -4.92057100 & 0.54623600 & -1.2099670 \\
\hline $\mathrm{C}$ & -5.50966700 & -0.22343300 & -0.19451200 \\
\hline $\mathrm{C}$ & 3. 80356000 & 1. 57905500 & -0.7297910 \\
\hline $\mathrm{C}$ & 4. 31952800 & 1. 50052400 & 0.5651660 \\
\hline $\mathrm{C}$ & 4. 09263200 & 0.55990700 & -1.64986400 \\
\hline $\mathrm{C}$ & 5. 14923700 & 0.43915000 & 0.9577760 \\
\hline $\mathrm{C}$ & 4. 89113300 & -0.52868200 & -1.29258200 \\
\hline $\mathrm{C}$ & 5. 41753100 & -0.55667600 & 0.01261900 \\
\hline $\mathrm{C}$ & -1.49400400 & -2.90681000 & 1. 703687 \\
\hline $\mathrm{C}$ & 0.49483200 & -3.82271500 & 2. 58106100 \\
\hline $\mathrm{C}$ & 1. 26554800 & -2.97451000 & 1.76024600 \\
\hline $\mathrm{C}$ & 0.61481500 & -2.36645400 & 0.592444 \\
\hline $\mathrm{C}$ & -0.74411900 & -2.38538200 & $0.491068 c$ \\
\hline $\mathrm{H}$ & 0.46724600 & -5.19926900 & 4. 22040900 \\
\hline $\mathrm{H}$ & -1.66032800 & -2.11594400 & 2. 45654100 \\
\hline $\mathrm{C}$ & 1.08829600 & -4.54357600 & 3. 61850900 \\
\hline $\mathrm{C}$ & 2. 63703400 & -2.84413000 & 2. 02109400 \\
\hline $\mathrm{C}$ & 3. 22864200 & -3.54576400 & 3. 06887700 \\
\hline $\mathrm{C}$ & 2. 45303700 & -4.40004500 & 3. 86000900 \\
\hline $\mathrm{H}$ & 3. 22660400 & -2.19873600 & 1. 37911000 \\
\hline $\mathrm{H}$ & 4. 29172500 & -3.43792400 & 3. 26380400 \\
\hline $\mathrm{H}$ & 2. 91188500 & -4.95385600 & 4. 6748840 \\
\hline 0 & 1. 47853100 & -1.90678700 & -0.35062500 \\
\hline 0 & -0.83674300 & -4.00285700 & 2. 35508800 \\
\hline $\mathrm{C}$ & -1.51233100 & -2.08657700 & -0.77583500 \\
\hline $\mathrm{H}$ & -0.91358800 & -1.46252900 & -1.4425730 \\
\hline $\mathrm{H}$ & -2.41407300 & -1.49782800 & -0.554490 \\
\hline $\mathrm{C}$ & -1.96137800 & -3.34510000 & -1.58307200 \\
\hline $\mathrm{C}$ & -0.93410600 & -4.41832300 & -1.8583660 \\
\hline $\mathrm{C}$ & -3.04880100 & -4.19875900 & -0.95325000 \\
\hline $\mathrm{C}$ & -1.49721800 & -5.63626700 & -1.4332060 \\
\hline
\end{tabular}




\begin{tabular}{|c|c|c|c|}
\hline $\mathrm{N}$ & -2.79867000 & -5.45993800 & -0.88456900 \\
\hline $\mathrm{C}$ & 0.33344200 & -4.39314600 & -2.42330200 \\
\hline $\mathrm{C}$ & 1. 02907000 & -5.60304600 & -2.55694700 \\
\hline $\mathrm{H}$ & 2. 02612000 & -5.60067300 & -2.98894300 \\
\hline $\mathrm{C}$ & 0.46067200 & -6.81016900 & -2.13647600 \\
\hline $\mathrm{H}$ & 1. 02065800 & -7.73455700 & -2.25096500 \\
\hline $\mathrm{C}$ & -0.81635300 & -6.84185500 & -1.56682300 \\
\hline $\mathrm{H}$ & 0.79305700 & -3.46141300 & -2.74072500 \\
\hline $\mathrm{H}$ & -1.27090500 & -7.76982700 & -1.23374200 \\
\hline $\mathrm{H}$ & -2.47146200 & -3.29827700 & 1. 41674100 \\
\hline H & -3.99855200 & -3.79242800 & -0.60715200 \\
\hline $\mathrm{H}$ & 1. 09972200 & -1.14205000 & -0.84436700 \\
\hline $\mathrm{H}$ & -6.23460600 & -0.98292500 & -0.47285600 \\
\hline $\mathrm{H}$ & -4.05123900 & 1. 15093700 & 2.53986500 \\
\hline $\mathrm{H}$ & -3.54626200 & 2. 16889500 & -1.57692500 \\
\hline $\mathrm{H}$ & 6.05823800 & -1.38533800 & 0.29077000 \\
\hline $\mathrm{H}$ & 3. 66434200 & 0.63087200 & -2.64277200 \\
\hline $\mathrm{H}$ & 4. 09302800 & 2.30026800 & 1. 26359600 \\
\hline $\mathrm{C}$ & -5.94782300 & -0.88656900 & 2. 22827200 \\
\hline $\mathrm{C}$ & -7.46322000 & -0.57579200 & 2. 16985700 \\
\hline $\mathrm{H}$ & -8.00325000 & -1.16442100 & 2.92159000 \\
\hline $\mathrm{H}$ & -7.88866800 & -0.81465100 & 1. 18945700 \\
\hline $\mathrm{H}$ & -7.65350600 & 0.48523400 & 2. 36743200 \\
\hline $\mathrm{C}$ & -5.72945000 & -2.39224000 & 1. 95291900 \\
\hline $\mathrm{H}$ & -6.26776200 & -2.99691000 & 2. 69246000 \\
\hline $\mathrm{H}$ & -4.66746800 & -2.65409300 & 2. 01766400 \\
\hline $\mathrm{H}$ & -6.09076100 & -2.68712700 & 0.96212200 \\
\hline $\mathrm{C}$ & -5.45206600 & -0.58998900 & 3. 65677600 \\
\hline $\mathrm{H}$ & -5.64133700 & 0.44906900 & 3. 94906000 \\
\hline $\mathrm{H}$ & -4.37916300 & -0.78576500 & 3. 76558600 \\
\hline $\mathrm{H}$ & -5.97946400 & -1.23261300 & 4. 37055100 \\
\hline $\mathrm{C}$ & 5. 75159600 & 0.43459200 & 2.37671600 \\
\hline $\mathrm{C}$ & 6.62651100 & -0.80496400 & 2. 64322900 \\
\hline $\mathrm{H}$ & 6. 05705200 & -1.73639700 & 2.54689500 \\
\hline $\mathrm{H}$ & 7. 02168800 & -0.76405600 & 3. 66459500 \\
\hline $\mathrm{H}$ & 7. 48291400 & -0.85697500 & 1.96104300 \\
\hline $\mathrm{C}$ & 4. 61700500 & 0.46069300 & 3. 42787200 \\
\hline $\mathrm{H}$ & 3. 97860700 & -0.42538200 & 3. 34335200 \\
\hline $\mathrm{H}$ & 3. 97816100 & 1. 34295300 & 3. 31665000 \\
\hline $\mathrm{H}$ & 5. 03874800 & 0.48055700 & 4. 44053900 \\
\hline $\mathrm{C}$ & 5. 21762000 & -1.67444500 & -2.26995600 \\
\hline $\mathrm{C}$ & 6. 74171000 & -1.70520900 & -2.53465500 \\
\hline $\mathrm{H}$ & 6. 99369000 & -2.52431900 & -3.21963300 \\
\hline $\mathrm{H}$ & 7. 31227500 & -1.85514700 & -1.61190800 \\
\hline
\end{tabular}


7. 08101500

$-0.76661300$

$-2.98845400$

4. 77299900

$-3.02338700$

$-1.65563000$

5. 30713900

$-3.24598700$

$-0.72566100$

$\mathrm{H}$

4. 97797500

$-3.84339900$

$-2.35551200$

3. 70165700

$-3.01647400$

$-1.42989700$

4. 49896900

$-1.51011400$

$-3.62263900$

4. 80330900

$-0.59341400$

$-4.14077300$

3. 40994400

$-1.49015900$

$-3.50281000$

4. 74684100

$-2.35436400$

$-4.27600000$

$-5.33645200$

0. 32607900

$-2.67884000$

$-5.09015100$

$-1.14453400$

$-3.08686600$

$-4.02482200$

$-1.39333900$

$-3.02329600$

$-5.41366700$

$-1.31194200$

-4. 12124400

$-5.63893600$

$-1.84724800$

$-2.45133700$

$-4.55047300$

1. 22560400

$-3.65193700$

$-3.47071900$

1. 04972500

$-3.58834700$

$-4.73524000$

2. 28925800

$-3.46474400$

$-4.86207100$

1. 01475500

$-4.68116600$

$-6.84240100$

0. 64952000

$-2.83068400$

$-7.46223800$

0. 00922900

-2. 19417800

$-7.16231800$

0. 49924000

$-3.86914200$

$-7.04824800$

1. 69088600

$-2.55820900$

6. 63958000

1. 69011900

2. 55390400

6. 06673500

2. 61460700

2. 42687100

7. 45592500

1. 69992900

1. 82242400

7. 08163800

1. 70631900

3. 55788100

$-2.36616300$

$-2.96087400$

$-2.53430700$ 
TS-III'-dt-R (Table S6, entry 7)

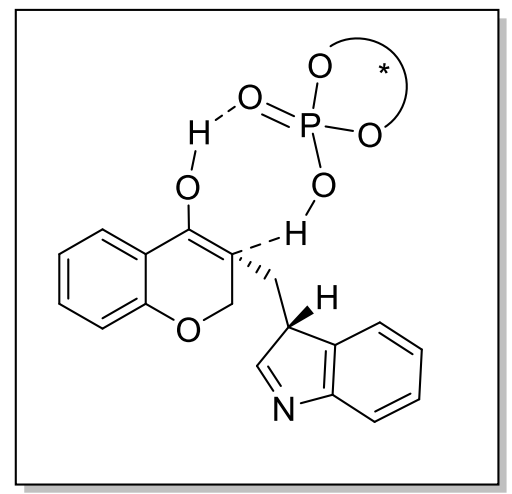

SCF energy [B3LYP-GD3BJ/6-311+G**/SMD (Chlorobenzene)]: -3291.644475860 a.u.

Thermal correction to Gibbs free energy at $258 \mathrm{~K}: 1.111300000$ a.u.

Gibbs free energy at $258 \mathrm{~K}$ [B3LYP-GD3BJ/6-311+G**/SMD (Chlorobenzene)]: -3290.533175860 a.u.

01

$\begin{array}{lrrr}\text { P } & -0.21289200 & 1.31967300 & -0.22748300 \\ 0 & 0.86529400 & 2.36058000 & 0.44008800 \\ 0 & -1.41569000 & 2.27851200 & -0.77057900 \\ 0 & -0.71503700 & 0.47009700 & 0.96033600 \\ 0 & 0.35654500 & 0.58602900 & -1.40997100 \\ \mathrm{C} & 1.51501100 & 3.23140200 & -0.44828100 \\ \mathrm{C} & 2.79027200 & 2.90275000 & -0.95103500 \\ \mathrm{C} & 3.31899600 & 3.72260000 & -1.96329800 \\ \mathrm{C} & 2.62146200 & 4.81871900 & -2.46836500 \\ \mathrm{C} & 1.40064300 & 5.16037600 & -1.89787200 \\ \mathrm{C} & 0.47202000 & 6.30207100 & -2.25161600 \\ \mathrm{C} & -0.83113900 & 5.91512300 & -1.51811600 \\ \mathrm{C} & -0.35520500 & 5.10013000 & -0.27006700 \\ \mathrm{C} & 0.86511400 & 4.39620300 & -0.84959100 \\ \mathrm{C} & 0.06549600 & 6.08166800 & 0.87692000 \\ \mathrm{C} & -1.25279300 & 6.43450500 & 1.59806500 \\ \mathrm{C} & -2.08173300 & 5.18629800 & 1.39149300 \\ \mathrm{C} & -3.24917200 & 4.79706500 & 2.03830800 \\ \mathrm{C} & -3.85236900 & 3.59406600 & 1.67756000 \\ \mathrm{C} & -3.28751000 & 2.72839500 & 0.72343500 \\ \mathrm{C} & -2.07796600 & 3.13151200 & 0.12254200 \\ \mathrm{C} & -1.51268400 & 4.37331500 & 0.40013600 \\ \mathrm{H} & -0.49040300 & -0.73281200 & 0.83050100 \\ \mathrm{H} & 4.30459500 & 3.48669500 & -2.35339700\end{array}$




\begin{tabular}{|c|c|c|c|}
\hline $\mathrm{H}$ & 3. 04164100 & 5. 40655400 & -3.28064700 \\
\hline H & 0.86628100 & 7. 26187900 & -1.88859000 \\
\hline H & 0.33192100 & 6. 40957000 & -3.33336300 \\
\hline $\mathrm{H}$ & -1.44909600 & 6. 77587800 & -1.2432560 \\
\hline $\mathrm{H}$ & -1.43484300 & 5. 25979200 & -2.155968 \\
\hline H & 0.60494800 & 6. 95600200 & 0.499340 \\
\hline H & 0.73243600 & 5. 55061800 & 1.5656160 \\
\hline H & -1.73551400 & 7. 31152000 & 1.1436450 \\
\hline $\mathrm{H}$ & -1.10401400 & 6. 67234800 & 2. 657675 \\
\hline H & -3.69823400 & 5. 42515300 & 2.803764 \\
\hline H & -4.79248500 & 3. 30653700 & 2.1382560 \\
\hline $\mathrm{C}$ & -4.00533400 & 1. 47574300 & 0.367480 \\
\hline $\mathrm{C}$ & -4.61424700 & 0.71068000 & 1. 371699 \\
\hline $\mathrm{C}$ & -4.17685300 & 1. 10221000 & -0.972849 \\
\hline $\mathrm{C}$ & -5.39654000 & -0.40716700 & 1. 065236 \\
\hline $\mathrm{C}$ & -4.94559700 & -0.01230900 & -1.322944 \\
\hline $\mathrm{C}$ & -5.54029500 & -0.74844200 & -0.287289 \\
\hline $\mathrm{C}$ & 3. 63542400 & 1. 80470100 & -0.406071 \\
\hline $\mathrm{C}$ & 3. 83491900 & 1. 68599800 & 0.973192 \\
\hline $\mathrm{C}$ & 4. 36806800 & 0.97472100 & -1.2721050 \\
\hline $\mathrm{C}$ & 4. 77042200 & 0.78537100 & 1.505010 \\
\hline $\mathrm{C}$ & 5. 31242600 & 0.06578100 & -0.787236 \\
\hline $\mathrm{C}$ & 5. 49686900 & -0.00609800 & 0.606762( \\
\hline C & -0.35208700 & -2.86672800 & 1. 9175140 \\
\hline $\mathrm{C}$ & -2.04576000 & -3.94523100 & 0.690055 \\
\hline $\mathrm{C}$ & -1.47476900 & -3.54067100 & -0.5348430 \\
\hline $\mathrm{C}$ & 0.06513400 & -2.03165600 & 0.711671 \\
\hline H & -3.44928900 & -5.23325900 & 1. 6702060 \\
\hline H & 0.28874200 & -3.74993000 & 2. 0428360 \\
\hline $\mathrm{C}$ & -3.02035200 & -4.94618900 & 0.716296 \\
\hline $\mathrm{C}$ & -1.89185900 & -4.15907700 & -1.7270500 \\
\hline $\mathrm{C}$ & -2.84210200 & -5.17046700 & -1.698028 \\
\hline $\mathrm{C}$ & -3.40789400 & -5.55495300 & -0.473855 \\
\hline H & -1.44948500 & -3.83526400 & -2.662589 \\
\hline $\mathrm{H}$ & -3.14880600 & -5.65721900 & -2.618560 \\
\hline H & -4.15477300 & -6.34395200 & -0.448058 \\
\hline 0 & -0.24141900 & -1.90789700 & -1.6771620 \\
\hline 0 & -1.71606700 & -3.32762300 & 1. 852282 \\
\hline $\mathrm{C}$ & 1.53276000 & -1.57356800 & 0.7380120 \\
\hline H & 1. 68129000 & -0.89766500 & 1.588271 \\
\hline H & 1. 73764700 & -0.96737600 & -0.145909 \\
\hline $\mathrm{C}$ & 2. 62669700 & -2.67737700 & 0.8156760 \\
\hline $\mathrm{C}$ & 2. 56524600 & -3.85882100 & -0.1233330 \\
\hline & 2. 79610600 & -3.41617000 & 2. 131719 \\
\hline
\end{tabular}


2. $75174200-5.01206000$

2. $89478800-4.69850500$

2. $41308900-3.97229700$

2. 44031800

2. 31616500

2. 62483300

2. 64325400

2. 78553100

2. 26000100

2. 93350300

$-0.29740800$

2. 87548200

0. 12787100

3. 27635300

6. 24425300

4. 18801000

$-3.71428100$

$-6.13851500$

$-4.45680600$

5. 01778400

5. 62625000

6. 57583600

5. 81798400

4. 95497800

5. 99204900

6. 98804300

5. 62692200

6. 10828900

3. 68700100

3. 24091500

2. 94925000

3. 86063300

$-5.15967500$

$-6.09288400$

$-6.67303000$

$-7.21099200$

$-6.83510100$

$-7.12716700$

-4. 55508900

$-5.01122900$

$-3.47862400$

$-4.70721400$

$-4.48697000$

$-3.40253300$
$-5.25181100$

$-5.35848700$

$-6.39115100$

$-7.37143100$

$-6.28435800$

$-3.09767300$

$-7.15829200$

$-2.27300900$

$-2.91164800$

$-0.95889000$

2. 34022800

$-0.68821100$

1. 06446700

1. 70903900

$-1.61756700$

1. 00051500

0. 75537100

2. 11184200

2. 29677100

2. 11896400

2. 94652500

-0. 36287000

$-0.22355700$

$-1.35396000$

-0. 36217000

0. 53622600

$-0.43042700$

1. 31311800

0. 54299300

$-0.43664900$

$-1.25005000$

-0. 47203900

$-1.18280900$

$-0.77169600$

0. 51511000

$-1.84309100$

$-2.59019600$

$-1.83742700$

$-2.17029400$

0. 52889700

0. 57830600
0. 66475200

2. 04442600

$-1.50062900$

$-2.07379300$

-3. 14804100

$-1.28295600$

$-1.75168000$

0. 10218500

$-2.12655800$

0. 72914300

2. 83352200

3. 09405900

$-1.59147400$

1. 63393800

0. 99437100

$-2.33689400$

$-1.73978300$

$-0.54429900$

2. 40363900

3. 02726100

3. 46011600

2. 94497800

4. 54037500

3. 23256100

3. 44539400

3. 01055900

3. 15173100

4. 53504000

3. 78357900

3. 52180400

3. 56059800

4. 86641600

$-2.78940000$

2. 15194900

-3. 10828100

$-2.47225900$

$-4.15121100$

$-2.96414300$

$-3.00539100$

$-2.34746700$

$-2.80322800$

$-4.04191400$

$-3.78437000$

$-3.63714000$ 
$-3.70234100$

$-4.66664800$

0. 18397200

$-4.80922100$

$-7.61652600$

$-1.28237900$

1. 88549000

$-8.12497300$

$-1.87866900$

2. 65333800

$-7.85332500$

$-1.72416000$

0. 91197200

$-8.03994900$

$-0.27150200$

1. 90433300

$-5.87097900$

$-0.68335700$

3. 56758600

$-6.26051800$

0. 33663800

3. 66514000

$-4.81023800$

$-0.67247100$

3. 84149300

-6. 39411600

$-1.30848400$

4. 30030000

$-5.53452900$

$-2.69103400$

2. 12224100

$-5.69348800$

-3. 16848500

1. 14968600

$-6.02818200$

$-3.30833900$

2. 88398100

-4. 45774700

$-2.69389200$

2. 32294800

$-0.49401600$

$-2.46521500$

$-0.51233500$

6. 19674000

$-0.78521100$

$-1.72157000$

6. 17182800

$-2.27286700$

$-1.30113000$

6. 52690200

$-2.42101700$

$-0.27607100$

6. 82365900

$-2.85987700$

$-1.95941900$

5. 16201000

$-2.69186800$

$-1.37191200$

5. 74276400

$-0.70365000$

$-3.19196700$

5. 82157200

0. 31334900

$-3.59165200$

4. 70690500

$-1.03965800$

$-3.31621300$

6. 37842800

$-1.34780600$

$-3.80993800$

7. 65095700

$-0.26048400$

$-1.63264900$

8. 30956200

$-0.84218800$

$-2.28957200$

8. 04359300

$-0.33381000$

$-0.61269200$

7. 70792300

0. 79077700

$-1.93748900$

3. 57289900

-2. 13341100

0. 65212500 
TS-III'-dt-S (Table S6, entry 8)

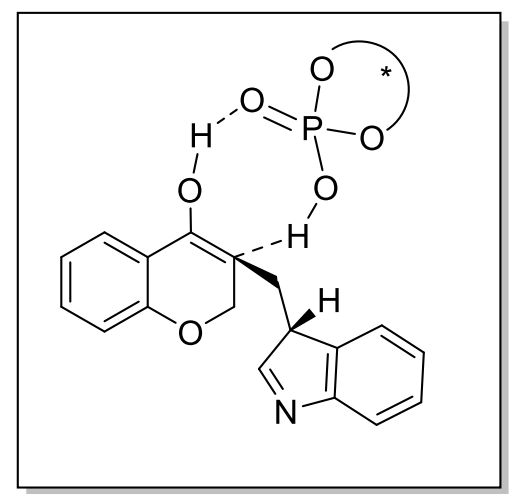

SCF energy [B3LYP-GD3BJ/6-311+G**/SMD (Chlorobenzene)]: -3291.643578360 a.u.

Thermal correction to Gibbs free energy at $258 \mathrm{~K}: 1.109963000$ a.u.

Gibbs free energy at $258 \mathrm{~K}$ [B3LYP-GD3BJ/6-311+G**/SMD (Chlorobenzene)]: -3290.533615360 a.u.

\begin{tabular}{|c|c|c|c|}
\hline $\mathrm{P}$ & 0.03896200 & 1. 28975000 & -0.02316000 \\
\hline 0 & -1.03184400 & 2. 43675200 & -0.48894200 \\
\hline 0 & 1. 31880000 & 2. 13727000 & 0.54359800 \\
\hline 0 & 0.40800600 & 0.58830500 & -1.34940300 \\
\hline 0 & -0.45598400 & 0.41779200 & 1. 09627500 \\
\hline $\mathrm{C}$ & -1.51723400 & 3. 29308800 & 0.50804400 \\
\hline $\mathrm{C}$ & -2.71324100 & 2. 98456400 & 1. 18347800 \\
\hline $\mathrm{C}$ & -3.08312500 & 3. 80965700 & 2. 25906200 \\
\hline $\mathrm{C}$ & -2.30513000 & 4. 89626300 & 2. 65757800 \\
\hline $\mathrm{C}$ & -1.15785000 & 5. 20608300 & 1. 93510300 \\
\hline $\mathrm{C}$ & -0.15741300 & 6. 31736900 & 2. 16696500 \\
\hline $\mathrm{C}$ & 1. 04621700 & 5. 86624700 & 1. 31089400 \\
\hline $\mathrm{C}$ & 0.41495200 & 5. 05488000 & 0.12765100 \\
\hline $\mathrm{C}$ & -0.77774300 & 4. 42598300 & 0.83331800 \\
\hline $\mathrm{C}$ & -0.03755600 & 6. 03234200 & -1.00755900 \\
\hline C & 1. 24306800 & 6. 29966500 & -1.82871500 \\
\hline $\mathrm{C}$ & 2. 02311900 & 5. 01511400 & -1.64953000 \\
\hline $\mathrm{C}$ & 3. 12632900 & 4. 55739900 & -2.36145200 \\
\hline $\mathrm{C}$ & 3. 70167200 & 3. 33818800 & -2.00577500 \\
\hline $\mathrm{C}$ & 3. 15556400 & 2. 52119400 & -1.00067000 \\
\hline $\mathrm{C}$ & 1. 99062400 & 2. 97860700 & -0.35332200 \\
\hline $\mathrm{C}$ & 1. 47636800 & 4. 24749300 & -0.60988700 \\
\hline $\mathrm{H}$ & 0.27090700 & -0.63069200 & -1.27237700 \\
\hline $\mathrm{H}$ & -4.00541200 & 3. 58380500 & 2. 78655100 \\
\hline $\mathrm{H}$ & -2.60216900 & 5. 49517000 & 3. 51493800 \\
\hline
\end{tabular}




\begin{tabular}{|c|c|c|c|}
\hline H & -0.55192000 & 7. 28439300 & 1. 82352000 \\
\hline $\mathrm{H}$ & 0.09965000 & 6. 44068700 & 3. 22523500 \\
\hline $\mathrm{H}$ & 1. 66910000 & 6. 69637300 & 0.96253300 \\
\hline $\mathrm{H}$ & 1.68323900 & 5. 19390200 & 1. 89659400 \\
\hline H & -0.50139700 & 6. 94222500 & -0.61343400 \\
\hline $\mathrm{H}$ & -0.77886900 & 5. 52323100 & -1.63382000 \\
\hline $\mathrm{H}$ & 1. 79999300 & 7. 16042600 & -1.43221500 \\
\hline $\mathrm{H}$ & 1.03144300 & 6. 52061300 & -2.88123200 \\
\hline $\mathrm{H}$ & 3. 55262900 & 5. 14774300 & -3.16877200 \\
\hline $\mathrm{H}$ & 4. 60413400 & 3. 00172100 & -2.50757400 \\
\hline C & 3. 88347200 & 1. 28290300 & -0.60658200 \\
\hline $\mathrm{C}$ & 4. 38874700 & 0.41217300 & -1.58305400 \\
\hline $\mathrm{C}$ & 4. 21248400 & 1. 06335800 & 0.73886500 \\
\hline $\mathrm{C}$ & 5. 23811200 & -0.64830500 & -1.24391100 \\
\hline $\mathrm{C}$ & 5. 07076600 & 0.02579200 & 1. 12154800 \\
\hline $\mathrm{C}$ & 5. 56834600 & -0.81210400 & 0.11090800 \\
\hline $\mathrm{C}$ & -3.61513200 & 1. 87342700 & 0.77428000 \\
\hline $\mathrm{C}$ & -4.06995900 & 1. 78418100 & -0.54343400 \\
\hline $\mathrm{C}$ & -4.10597600 & 0.96938600 & 1. 73003500 \\
\hline $\mathrm{C}$ & -5.02706200 & 0.83208300 & -0.92533000 \\
\hline $\mathrm{C}$ & -5.04715100 & -0.00408400 & 1. 39071900 \\
\hline $\mathrm{C}$ & -5.49901100 & -0.04490900 & 0.05786500 \\
\hline $\mathrm{C}$ & 0.57651100 & -2.56604600 & -2.47867300 \\
\hline C & -1.38226000 & -3.84116900 & -2.60783500 \\
\hline C & -2.01521800 & -2.94637300 & -1.70894200 \\
\hline C & -1.16801600 & -2.17245400 & -0.81646900 \\
\hline C & 0.20946500 & -2.01662200 & -1.10745000 \\
\hline H & -1.63021000 & -5.46893400 & -3.98076200 \\
\hline H & 0.27398600 & -1.87084400 & -3.27382600 \\
\hline C & -2.14037400 & -4.78330600 & -3.31239500 \\
\hline C & -3.41813800 & -2.98563500 & -1.57440600 \\
\hline $\mathrm{C}$ & -4.16836600 & -3.89744300 & -2.30072500 \\
\hline C & -3.52167300 & -4.80141600 & -3.15962200 \\
\hline H & -3.88796100 & -2.29436500 & -0.88352800 \\
\hline H & -5.24884600 & -3.92199100 & -2.19820900 \\
\hline H & -4.10770500 & -5.52248600 & -3.72322800 \\
\hline 0 & -1.75439000 & -1.67668100 & 0.24792500 \\
\hline 0 & -0.04088500 & -3.84075000 & -2.78200100 \\
\hline C & 1. 25444400 & -2.17382500 & 0.00982300 \\
\hline H & 0.92159100 & -1.66507600 & 0.91764300 \\
\hline H & 2. 16386600 & -1.65194800 & -0.31182700 \\
\hline C & 1. 64256000 & -3.64311500 & 0.35858800 \\
\hline C & 0.72885600 & -4.41653100 & 1. 28876000 \\
\hline & 2. 91073400 & -3.65717200 & 1. 19438100 \\
\hline
\end{tabular}




\begin{tabular}{|c|c|c|c|}
\hline C & 1. 48849200 & -4.72050700 & 2. 43663300 \\
\hline $\mathrm{N}$ & 2. 82076800 & -4.23206900 & 2. 34199000 \\
\hline $\mathrm{C}$ & -0.58377600 & -4.86189000 & 1. 19463600 \\
\hline $\mathrm{C}$ & -1.13089400 & -5.58153900 & 2. 26687100 \\
\hline $\mathrm{H}$ & -2.15892700 & -5.92839600 & 2. 20578100 \\
\hline $\mathrm{C}$ & -0.37300700 & -5.86163200 & 3. 40764200 \\
\hline $\mathrm{H}$ & -0.81873600 & -6.42271300 & 4. 22473000 \\
\hline C & 0.95568600 & -5.43597400 & 3. 50336300 \\
\hline $\mathrm{H}$ & -1.18738000 & -4.67272600 & 0.31302000 \\
\hline H & 1.56567500 & -5.65633400 & 4. 37404500 \\
\hline $\mathrm{H}$ & 1. 65045300 & -2.74026700 & -2.56550600 \\
\hline H & 3. 83154600 & -3.17642300 & 0.87180700 \\
\hline H & -1.21123900 & -0.93153400 & 0.68648000 \\
\hline $\mathrm{H}$ & 6. 25581900 & -1.60655200 & 0.38631700 \\
\hline $\mathrm{H}$ & 4. 11287500 & 0.58716400 & -2.61650100 \\
\hline H & 3. 81882700 & 1. 74702900 & 1. 48016000 \\
\hline H & -6.24634100 & -0.78349200 & -0.20996600 \\
\hline $\mathrm{H}$ & -3.72303400 & 1. 03803900 & 2. 74128500 \\
\hline $\mathrm{H}$ & -3.68810400 & 2. 49201700 & -1.27178200 \\
\hline $\mathrm{C}$ & 5. 84633200 & -1.59274100 & -2.30100200 \\
\hline $\mathrm{C}$ & 7. 38781200 & -1.45516000 & -2.28086600 \\
\hline $\mathrm{H}$ & 7. 84090600 & -2.11679400 & -3.02948400 \\
\hline $\mathrm{H}$ & 7. 80723200 & -1.71963400 & -1.30467500 \\
\hline H & 7. 69151200 & -0.42670600 & -2.50675000 \\
\hline $\mathrm{C}$ & 5. 46507500 & -3.05782200 & -1.98562300 \\
\hline $\mathrm{H}$ & 5. 90904100 & -3.73676900 & -2.72386800 \\
\hline $\mathrm{H}$ & 4. 37729900 & -3.19590000 & -2.01512800 \\
\hline $\mathrm{H}$ & 5. 81540400 & -3.36997800 & -0.99638500 \\
\hline $\mathrm{C}$ & 5. 35804500 & -1.27224300 & -3.72687100 \\
\hline H & 5.64246500 & -0.26128900 & -4.03949200 \\
\hline $\mathrm{H}$ & 4. 26922800 & -1.36167000 & -3.81666000 \\
\hline $\mathrm{H}$ & 5.80726800 & -1.97501900 & -4.43772400 \\
\hline $\mathrm{C}$ & -5.53886600 & 0.81682800 & -2.37962400 \\
\hline $\mathrm{C}$ & -6.57260900 & -0.29651700 & -2.63293800 \\
\hline $\mathrm{H}$ & -6.15172400 & -1.29218200 & -2.45216200 \\
\hline $\mathrm{H}$ & -6.90064000 & -0.26351500 & -3.67810200 \\
\hline $\mathrm{H}$ & -7.46292100 & -0.17979100 & -2.00446200 \\
\hline $\mathrm{C}$ & -4.35159700 & 0.59993900 & -3.34775700 \\
\hline $\mathrm{H}$ & -3.86739300 & -0.36579000 & -3.16472800 \\
\hline $\mathrm{H}$ & -3.59004000 & 1. 37909100 & -3.24262900 \\
\hline $\mathrm{H}$ & -4.70135800 & 0.61223500 & -4.38767900 \\
\hline $\mathrm{C}$ & -5.60467400 & -1.00993200 & 2. 41699200 \\
\hline $\mathrm{C}$ & -7.13475300 & -0.81916500 & 2. 54670800 \\
\hline H & -7.55043000 & -1.53841700 & 3. 26335200 \\
\hline
\end{tabular}




\begin{tabular}{lrrr} 
H & -7.64805200 & -0.96674800 & 1.59054700 \\
$\mathrm{H}$ & -7.37444600 & 0.19001600 & 2.90122300 \\
$\mathrm{C}$ & -5.30523300 & -2.45256500 & 1.94461900 \\
$\mathrm{H}$ & -5.79233700 & -2.67715300 & 0.98915300 \\
$\mathrm{H}$ & -5.67255100 & -3.17908500 & 2.68013000 \\
$\mathrm{H}$ & -4.22777100 & -2.60627300 & 1.81858800 \\
$\mathrm{C}$ & -4.98005100 & -0.83011500 & 3.81391600 \\
$\mathrm{H}$ & -5.18127200 & 0.16520700 & 4.22591400 \\
$\mathrm{H}$ & -3.89517300 & -0.98163100 & 3.79755200 \\
$\mathrm{H}$ & -5.40729000 & -1.56520500 & 4.50543900 \\
$\mathrm{C}$ & 5.55575100 & -0.13878700 & 2.57755700 \\
$\mathrm{C}$ & 5.45452600 & -1.60899700 & 3.04168500 \\
$\mathrm{H}$ & 4.42052300 & -1.96743000 & 3.03942600 \\
$\mathrm{H}$ & 5.83728600 & -1.70311800 & 4.06509400 \\
$\mathrm{H}$ & 6.04504000 & -2.28546600 & 2.41490900 \\
$\mathrm{C}$ & 4.74136200 & 0.72230200 & 3.56342800 \\
$\mathrm{H}$ & 3.67210100 & 0.48694200 & 3.51956300 \\
$\mathrm{H}$ & 4.86370100 & 1.79440500 & 3.37380500 \\
$\mathrm{H}$ & 5.08452500 & 0.53185900 & 4.58658000 \\
$\mathrm{C}$ & 7.03686600 & 0.30935800 & 2.64622700 \\
$\mathrm{H}$ & 7.67217300 & -0.30529400 & 1.99876100 \\
$\mathrm{H}$ & 7.41634400 & 0.22184100 & 3.67189300 \\
$\mathrm{H}$ & 7.14749700 & 1.35304400 & 2.33000100 \\
$\mathrm{C}$ & -6.21321600 & 2.17369400 & -2.69581200 \\
$\mathrm{H}$ & -5.51774500 & 3.01108100 & -2.57876000 \\
$\mathrm{H}$ & -7.06547400 & 2.35176300 & -2.02992600 \\
$\mathrm{H}$ & -6.58037500 & 2.18591400 & -3.72963000 \\
$\mathrm{H}$ & 1.81419600 & -4.19634900 & -0.57737600 \\
& & & \\
\hline & &
\end{tabular}


TS-III-dt-R (Table S6, entry 9)

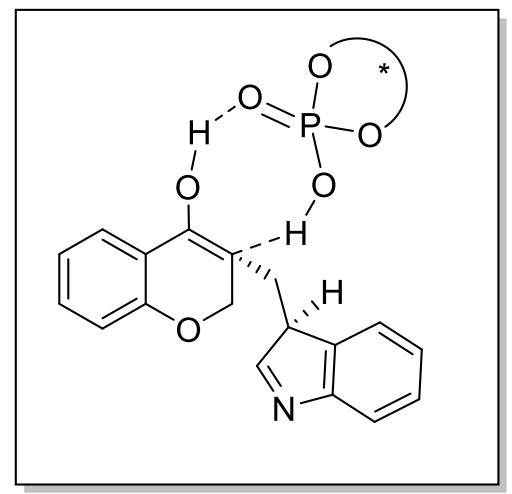

SCF energy [B3LYP-GD3BJ/6-311+G**/SMD (Chlorobenzene)]: -3291.638656620 a.u.

Thermal correction to Gibbs free energy at $258 \mathrm{~K}: 1.112100000$ a.u.

Gibbs free energy at $258 \mathrm{~K}$ [B3LYP-GD3BJ/6-311+G**/SMD (Chlorobenzene)]: -3290.526556620 a.u.

\begin{tabular}{|c|c|c|c|}
\hline $\mathrm{P}$ & -0.11186900 & 1. 19453500 & -0.24270200 \\
\hline 0 & 0.98059200 & 2. 26239800 & 0.34987200 \\
\hline 0 & -1.32254100 & 2. 12808800 & -0.82532400 \\
\hline 0 & -0.61098400 & 0.41174100 & 0.99148500 \\
\hline 0 & 0.43770000 & 0.38709000 & -1.38608100 \\
\hline $\mathrm{C}$ & 1. 64753800 & 3. 05808800 & -0.59865600 \\
\hline $\mathrm{C}$ & 2. 93331200 & 2. 69658900 & -1.04916700 \\
\hline $\mathrm{C}$ & 3. 46812900 & 3. 42727300 & -2.12568000 \\
\hline $\mathrm{C}$ & 2. 77069200 & 4. 46635300 & -2.73897500 \\
\hline $\mathrm{C}$ & 1. 54541000 & 4. 85611600 & -2.21064100 \\
\hline $\mathrm{C}$ & 0.62286000 & 5. 96858200 & -2.66143000 \\
\hline $\mathrm{C}$ & -0.67654000 & 5. 66784600 & -1.88057000 \\
\hline $\mathrm{C}$ & -0.19468300 & 4. 95689300 & -0.57353700 \\
\hline $\mathrm{C}$ & 1. 00561100 & 4. 18628600 & -1.10273000 \\
\hline $\mathrm{C}$ & 0.26949700 & 6. 02540000 & 0.47599400 \\
\hline $\mathrm{C}$ & -1.02324200 & 6. 46081900 & 1. 19562700 \\
\hline $\mathrm{C}$ & -1.87401800 & 5. 21329500 & 1. 11729900 \\
\hline $\mathrm{C}$ & -3.02663100 & 4. 90151200 & 1. 82814800 \\
\hline $\mathrm{C}$ & -3.66455200 & 3. 68994400 & 1. 57548500 \\
\hline $\mathrm{C}$ & -3.14895500 & 2. 74260200 & 0.67168400 \\
\hline $\mathrm{C}$ & -1.94381800 & 3. 06276200 & 0.01426900 \\
\hline $\mathrm{C}$ & -1.34805500 & 4. 31132600 & 0.18032200 \\
\hline $\mathrm{H}$ & -0.54662200 & -0.82064600 & 0.82488700 \\
\hline $\mathrm{H}$ & 4. 46245800 & 3. 16960500 & -2.47710700 \\
\hline $\mathrm{H}$ & 3. 19842200 & 4. 98019300 & -3.59628500 \\
\hline
\end{tabular}




\begin{tabular}{|c|c|c|c|}
\hline $\mathrm{H}$ & 1. 02975600 & 6.95482700 & -2.39712700 \\
\hline $\mathrm{H}$ & 0.47066200 & 5. 97328700 & -3.74696400 \\
\hline $\mathrm{H}$ & -1.27670600 & 6. 55987100 & -1.67396800 \\
\hline $\mathrm{H}$ & -1.29959100 & 4. 96982400 & -2.45092900 \\
\hline $\mathrm{H}$ & 0.81394600 & 6. 85516300 & 0.01438600 \\
\hline $\mathrm{H}$ & 0.94439400 & 5.54203800 & 1. 19148400 \\
\hline $\mathrm{H}$ & -1.50607600 & 7. 30216300 & 0.67804200 \\
\hline $\mathrm{H}$ & -0.84466200 & 6.78722500 & 2. 22670000 \\
\hline $\mathrm{H}$ & -3.44003900 & 5. 59704600 & 2. 55434900 \\
\hline $\mathrm{H}$ & -4.59964300 & 3. 46183300 & 2. 07767400 \\
\hline $\mathrm{C}$ & -3.94882900 & 1.51577300 & 0.41021600 \\
\hline $\mathrm{C}$ & -4.55790400 & 0.84254100 & 1. 47827700 \\
\hline $\mathrm{C}$ & -4.24085200 & 1. 11895400 & -0.90232800 \\
\hline $\mathrm{C}$ & -5.46966600 & -0.19560700 & 1. 26345500 \\
\hline $\mathrm{C}$ & -5.15144500 & 0.08893800 & -1.16202500 \\
\hline $\mathrm{C}$ & -5.74747900 & -0.55063500 & -0.06447300 \\
\hline $\mathrm{C}$ & 3. 78804100 & 1. 67664800 & -0.38374800 \\
\hline $\mathrm{C}$ & 3. 90992100 & 1. 65949500 & 1. 01267500 \\
\hline $\mathrm{C}$ & 4. 61424200 & 0.83550400 & -1.14115500 \\
\hline $\mathrm{C}$ & 4. 85695000 & 0.85256900 & 1. 65453500 \\
\hline C & 5. 57370800 & 0.01062000 & -0.54242200 \\
\hline $\mathrm{C}$ & 5. 67904900 & 0.04098600 & 0.85684200 \\
\hline $\mathrm{C}$ & -0.80022900 & -2.91838400 & 1. 90158400 \\
\hline $\mathrm{C}$ & -2.53367000 & -3.79758800 & 0.57070400 \\
\hline $\mathrm{C}$ & -1.85234900 & -3.46915400 & -0.62000700 \\
\hline $\mathrm{C}$ & -0.19292600 & -2.17148600 & 0.71698000 \\
\hline $\mathrm{H}$ & -4.11954000 & -4.92372500 & 1. 47048500 \\
\hline $\mathrm{H}$ & -0.28801000 & -3.87495200 & 2. 08914700 \\
\hline $\mathrm{C}$ & -3.60827200 & -4.69034900 & 0.54319500 \\
\hline $\mathrm{C}$ & -2.26509900 & -4.04761600 & -1.83418600 \\
\hline $\mathrm{C}$ & -3.31693500 & -4.95326100 & -1.85676200 \\
\hline $\mathrm{C}$ & -3.98881200 & -5.26541600 & -0.66614200 \\
\hline $\mathrm{H}$ & -1.73635500 & -3.78009700 & -2.74261000 \\
\hline $\mathrm{H}$ & -3.62220100 & -5.41119300 & -2.79236500 \\
\hline $\mathrm{H}$ & -4.81489500 & -5.97125100 & -0.68135400 \\
\hline 0 & -0.34383700 & -2.02820100 & -1.68220100 \\
\hline 0 & -2.20135700 & -3.21120700 & 1. 74922400 \\
\hline $\mathrm{C}$ & 1. 33149600 & -1.96584300 & 0.82281900 \\
\hline $\mathrm{H}$ & 1. 53330200 & -1.19774600 & 1. 57837800 \\
\hline $\mathrm{H}$ & 1.71373100 & -1.56594200 & -0.11928000 \\
\hline $\mathrm{C}$ & 2. 16120000 & -3.22589900 & 1.21516800 \\
\hline $\mathrm{C}$ & 2. 24349500 & -4.39634800 & 0.25580800 \\
\hline $\mathrm{C}$ & 3. 63775400 & -2.88678800 & 1. 31892100 \\
\hline & 3. 61010700 & -4.58065200 & -0.03590800 \\
\hline
\end{tabular}


4. $43065700-3.63173500$

1. $30317000-5.26499600$

1. $74068200-6.28963800$

1. 01136100

3. 09744300

3. 41235600

4. 05431300

0. 24532700

5. 11398900

$-0.73276500$

4. 01491200

0. 12147600

3. 27872600

6. 42247600

4. 50395200

$-3.77249400$

$-6.46256800$

$-4.30769600$

5. 00012100

5. 42638900

6. 38543900

5. 53773900

4. 68846600

6. 05789700

7. 05807700

5. 82095700

6. 10578500

3. 64763900

3. 33565000

2. 84754100

3. 73247900

$-5.55423800$

-6. 18326300

$-7.06538300$

$-7.67708400$

$-7.37385800$

$-7.29691600$

$-5.27015100$

$-5.80860900$

$-4.20228300$

$-5.57830000$

$-4.78248400$

$-3.70032300$

$-4.98301000$
$-6.96789800$

-6. 45112100

$-7.25354900$

$-5.59611400$

$-5.17254800$

$-5.71450000$

$-2.31626100$

$-2.05591100$

$-1.11753500$

2. 32196900

$-0.58266600$

0. 84050600

1. 65460300

$-1.34646000$

1. 15155000

0. 92914900

2. 36384800

2. 62671400

2. 44403400

3. 10808300

$-0.04812300$

0. 16988300

$-1.09154100$

0. 03621600

0. 60192400

$-0.42670700$

1. 26937000

0. 70033200

$-0.31321900$

$-0.92072100$

$-0.04344900$

$-0.62011200$

$-0.31832100$

1. 01731400

$-1.81530800$

$-2.44710500$

$-2.03115100$

$-2.11522500$

0. 47984400

0. 32882100

1. 55532800
0. 63222900

$-0.28556300$

$-1.13761700$

$-1.57211400$

$-1.43174300$

$-2.09340100$

$-0.87558500$

$-0.06240300$

$-1.07961800$

2. 81136400

1. 90883800

$-1.57712300$

1. 59501300

1. 33411200

$-2.22115900$

$-1.71813200$

$-0.25177800$

2. 48609700

3. 18920100

3. 58529100

3. 12463200

4. 67394900

3. 26853400

3. 73747700

3. 34685600

3. 49839700

4. 82922200

3. 86520900

3. 64718700

3. 53021500

4. 95458700

$-2.59504700$

2. 42183600

$-2.79233500$

$-2.09034900$

$-3.80883900$

$-2.64241100$

$-2.81919400$

$-2.10525000$

$-2.71132000$

$-3.82883100$

$-3.66737800$

$-3.58599200$

$-3.60461200$ 
$-4.66476300$

$-8.23732400-1.26029500$

3. 08939200

$-8.08263600$

$-1.16423300$

1. 32893300

$-7.99352200$

0. 31120000

2. 30131200

$-5.77561600$

$-0.36856300$

3. 80140700

$-6.02637800$

0.69297900

3. 90787400

$-4.70214000$

$-0.48714400$

3. 98653200

$-6.30903300$

$-0.91387600$

4. 58848200

$-5.82934600$

$-2.42456100$

2. 38873300

$-6.12484000$

$-2.88877100$

1. 44194800

$-6.34439400$

$-2.95657700$

3. 19893900

$-4.75072800$

$-2.56929000$

2. 51190400

$-0.74008100$

$-2.53136100$

$-0.54064200$

6. 50315300

$-0.84398800$

$-1.42807900$

7. 41937200

$-1.76896400$

$-0.60324800$

8. 09312400

$-1.20213200$

0. 05035000

8. 04756100

$-2.35847500$

$-1.28137600$

6. 83812200

$-2.46795900$

0. 00740500

5. 66512700

$-1.73125800$

$-2.37813800$

5. 01379700

$-1.13945500$

$-3.02983500$

5. 03834700

$-2.42534700$

$-1.81002900$

6. 32799500

$-2.32148600$

$-3.02286200$

7. 39422500

0. 10129600

$-2.26994200$

8. 06753200

$-0.48029800$

$-2.91210300$

8. 00808800

0. 74093000

$-1.62509500$

6. 79686700

0. 75363500

$-2.91665500$

1. 82919200

$-3.56966600$

2. 20704900 
TS-III-dt-S (Table S6, entry 10)

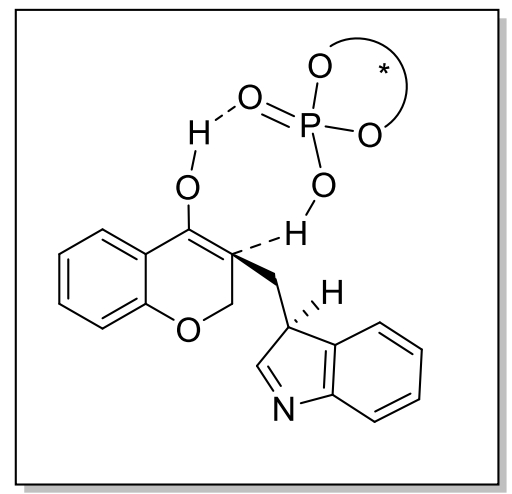

SCF energy [B3LYP-GD3BJ/6-311+G**/SMD (Chlorobenzene)]: -3291.647469050 a.u.

Thermal correction to Gibbs free energy at $258 \mathrm{~K}: 1.110047000$ a.u.

Gibbs free energy at $258 \mathrm{~K}$ [B3LYP-GD3BJ/6-311+G**/SMD (Chlorobenzene)]: -3290.537422050 a.u.

\begin{tabular}{|c|c|c|c|}
\hline P & 0.20731500 & 1. 38972100 & 0.08416200 \\
\hline 0 & -0.75904100 & 2. 54154500 & -0.56103800 \\
\hline 0 & 1.51014900 & 2. 21575000 & 0.62852600 \\
\hline 0 & 0.60050400 & 0.52123900 & -1.13400900 \\
\hline 0 & -0.39768900 & 0.67937300 & 1. 26123900 \\
\hline $\mathrm{C}$ & -1.27798700 & 3. 48817000 & 0.33488000 \\
\hline $\mathrm{C}$ & -2.54100700 & 3. 28310400 & 0.92316300 \\
\hline $\mathrm{C}$ & -2.94822500 & 4. 19021700 & 1. 91647100 \\
\hline $\mathrm{C}$ & -2.14064200 & 5. 25090300 & 2. 32491500 \\
\hline $\mathrm{C}$ & -0.92180100 & 5. 45541400 & 1. 68748000 \\
\hline $\mathrm{C}$ & 0.12382900 & 6. 51715700 & 1. 95098000 \\
\hline $\mathrm{C}$ & 1. 35896300 & 5. 96143800 & 1. 20797800 \\
\hline $\mathrm{C}$ & 0.76813900 & 5. 13426900 & 0.01625100 \\
\hline $\mathrm{C}$ & -0.50361100 & 4. 59828000 & 0.65886400 \\
\hline $\mathrm{C}$ & 0.43708800 & 6. 08815700 & -1.18085500 \\
\hline $\mathrm{C}$ & 1. 77587400 & 6. 25761400 & -1.93025500 \\
\hline $\mathrm{C}$ & 2. 46991800 & 4. 94034500 & -1.66154500 \\
\hline $\mathrm{C}$ & 3. 57839600 & 4. 39376800 & -2.29806400 \\
\hline $\mathrm{C}$ & 4. 05620500 & 3. 15417000 & -1.87708900 \\
\hline $\mathrm{C}$ & 3. 41399300 & 2. 40697900 & -0.87387500 \\
\hline $\mathrm{C}$ & 2. 25774400 & 2. 96368700 & -0.29026700 \\
\hline $\mathrm{C}$ & 1.82856500 & 4. 24635500 & -0.62410200 \\
\hline $\mathrm{H}$ & 0.42770800 & -0.65768500 & -0.92547300 \\
\hline $\mathrm{H}$ & -3.92297800 & 4. 04788000 & 2. 3738540 \\
\hline $\mathrm{H}$ & -2.46945300 & 5. 91169300 & 3. 12319800 \\
\hline
\end{tabular}




\begin{tabular}{|c|c|c|c|}
\hline $\mathrm{H}$ & -0.18472500 & 7. 49000100 & 1.54241700 \\
\hline $\mathrm{H}$ & 0.30843200 & 6. 67037000 & 3. 02045700 \\
\hline H & 2. 05292700 & 6. 73937000 & 0.87374900 \\
\hline $\mathrm{H}$ & 1.91134400 & 5.28207400 & 1. 8670370 \\
\hline $\mathrm{H}$ & -0.00099600 & 7. 03527700 & -0.8503030 \\
\hline $\mathrm{H}$ & -0.29132800 & 5. 59296200 & -1.832900 \\
\hline H & 2. 35565500 & 7. 10058600 & -1.5281580 \\
\hline H & 1. 64013000 & 6. 45191700 & -3.0003900 \\
\hline $\mathrm{H}$ & 4. 07981100 & 4. 92855300 & -3.100885 \\
\hline H & 4. 95575800 & 2. 74495500 & -2.3271090 \\
\hline $\mathrm{C}$ & 4. 02282100 & 1. 12070100 & -0.4365390 \\
\hline $\mathrm{C}$ & 4. 52418100 & 0.21986500 & -1.387396 \\
\hline $\mathrm{C}$ & 4. 23081100 & 0.85891700 & 0.925894 \\
\hline $\mathrm{C}$ & 5. 24757200 & -0.91703800 & -1.007785 \\
\hline $\mathrm{C}$ & 4. 95068100 & -0.26385300 & 1. 349188 \\
\hline $\mathrm{C}$ & 5. 44424800 & -1.13366500 & 0.364265 \\
\hline $\mathrm{C}$ & -3.46676900 & 2. 19359400 & 0.510669 \\
\hline $\mathrm{C}$ & -3.77798700 & 1. 99762600 & -0.8371210 \\
\hline $\mathrm{C}$ & -4.12249900 & 1. 41997400 & 1. 482493 \\
\hline $\mathrm{C}$ & -4.74904500 & 1. 06697900 & -1.2371050 \\
\hline $\mathrm{C}$ & -5.08546300 & 0.47371600 & 1. 128709 \\
\hline $\mathrm{C}$ & -5.38654700 & 0.32266000 & -0.238193 \\
\hline $\mathrm{C}$ & 0.73505600 & -2.75121900 & -1.8725190 \\
\hline C & -1.28352500 & -3.92606100 & -2.045256 \\
\hline $\mathrm{C}$ & -1.93041400 & -2.91268300 & -1.295674 \\
\hline $\mathrm{C}$ & -1.11228000 & -2.09484900 & -0.420371 \\
\hline $\mathrm{C}$ & 0.29021800 & -2.04041500 & -0.601006 \\
\hline H & -1.51938600 & -5.67624700 & -3.2600110 \\
\hline H & 0.54916100 & -2.12179900 & -2.754447 \\
\hline $\mathrm{C}$ & -2.03963200 & -4.89802700 & -2.711724 \\
\hline $\mathrm{C}$ & -3.33925700 & -2.86500000 & -1.276866 \\
\hline $\mathrm{C}$ & -4.08358800 & -3.80639400 & -1.9695000 \\
\hline $\mathrm{C}$ & -3.42638800 & -4.82824500 & -2.675611 \\
\hline H & -3.81954400 & -2.08225300 & -0.700678 \\
\hline $\mathrm{H}$ & -5.16845600 & -3.76471600 & -1.956090 \\
\hline H & -4.00874000 & -5.57330300 & -3.211387 \\
\hline 0 & -1.75655900 & -1.44499500 & 0.528257 \\
\hline 0 & 0.06166400 & -4.00818100 & -2.114202 \\
\hline $\mathrm{C}$ & 1. 20942500 & -2.09141600 & 0.630383 \\
\hline $\mathrm{H}$ & 0.82868200 & -1.40832700 & 1. 393588 \\
\hline H & 2. 19541100 & -1.69833000 & 0.354405 \\
\hline C & 1. 41550200 & -3.48439100 & 1. 2924200 \\
\hline $\mathrm{C}$ & 0.19655700 & -4.28122500 & 1. 6958900 \\
\hline & 2. 13393600 & -4.53527700 & 0.463603 \\
\hline
\end{tabular}




\begin{tabular}{|c|c|c|c|}
\hline C & 0.34739100 & -5.56380000 & 1. 13448500 \\
\hline $\mathrm{N}$ & 1. 55318800 & -5.68181900 & 0.38929000 \\
\hline $\mathrm{C}$ & -0.91001100 & -3.98797500 & 2. 48243100 \\
\hline C & -1.87015300 & -4.98895800 & 2. 68635700 \\
\hline $\mathrm{H}$ & -2.74579600 & -4.77448100 & 3. 29303700 \\
\hline $\mathrm{C}$ & -1.71601600 & -6.25856700 & 2. 11912400 \\
\hline $\mathrm{H}$ & -2.47408500 & -7.01789900 & 2. 29235000 \\
\hline C & -0.59826300 & -6.56411100 & 1. 33562200 \\
\hline $\mathrm{H}$ & -1.04489100 & -3.00468300 & 2. 92494600 \\
\hline H & -0.46040800 & -7.54696400 & 0.89544200 \\
\hline H & 1. 79715500 & -2.99654900 & -1.84456000 \\
\hline H & 3. 09678400 & -4.36043900 & -0.01337100 \\
\hline H & -1.21063700 & -0.68345000 & 0.91723200 \\
\hline $\mathrm{H}$ & 6. 02015900 & -1.99949000 & 0.67767000 \\
\hline $\mathrm{H}$ & 4. 34155500 & 0.42791200 & -2.43503000 \\
\hline H & 3. 84707000 & 1. 56669700 & 1. 64911100 \\
\hline H & -6.14677700 & -0.39647800 & -0.52215600 \\
\hline H & -3.85117300 & 1. 56880200 & 2. 52088100 \\
\hline $\mathrm{H}$ & -3.27018000 & 2. 60503300 & -1.57907700 \\
\hline $\mathrm{C}$ & 5.87327400 & -1.88197000 & -2.03575500 \\
\hline $\mathrm{C}$ & 7. 41483200 & -1.82033800 & -1.90732200 \\
\hline $\mathrm{H}$ & 7. 88665300 & -2.49593500 & -2.63149500 \\
\hline H & 7. 75152300 & -2.11316400 & -0.90725200 \\
\hline H & 7. 78198400 & -0.80562100 & -2.09882800 \\
\hline $\mathrm{C}$ & 5. 40237800 & -3.33045800 & -1.77094700 \\
\hline $\mathrm{H}$ & 5. 87618500 & -4.02242200 & -2.47756200 \\
\hline $\mathrm{H}$ & 4. 31680000 & -3.41958400 & -1.89640700 \\
\hline $\mathrm{H}$ & 5. 65482500 & -3.66801700 & -0.76020100 \\
\hline $\mathrm{C}$ & 5. 49877200 & -1.52127700 & -3.48615400 \\
\hline H & 5. 86489100 & -0.52804100 & -3.76867700 \\
\hline $\mathrm{H}$ & 4. 41457900 & -1.54316500 & -3.64511100 \\
\hline $\mathrm{H}$ & 5.94927800 & -2.24535500 & -4.17439400 \\
\hline $\mathrm{C}$ & -5.09376600 & 0.92965700 & -2.73352800 \\
\hline $\mathrm{C}$ & -6.16828800 & -0.14191200 & -2.99784300 \\
\hline H & -5.83987500 & -1.13664600 & -2.67600200 \\
\hline H & -6.37619800 & -0.19693000 & -4.07245700 \\
\hline H & -7.11223700 & 0.09075200 & -2.49156500 \\
\hline $\mathrm{C}$ & -3.82345800 & 0.54291700 & -3.52730800 \\
\hline H & -3.43496200 & -0.42670400 & -3.19681800 \\
\hline $\mathrm{H}$ & -3.02433100 & 1. 28089300 & -3.40538600 \\
\hline $\mathrm{H}$ & -4.05195500 & 0.47023400 & -4.59797200 \\
\hline $\mathrm{C}$ & -5.81917100 & -0.39029500 & 2. 17358700 \\
\hline $\mathrm{C}$ & -7.34220100 & -0.13399000 & 2. 08125000 \\
\hline H & -7.87773300 & -0.74574100 & 2. 81785000 \\
\hline
\end{tabular}




\begin{tabular}{lrrr} 
H & -7.73927800 & -0.38278800 & 1.09150200 \\
$\mathrm{H}$ & -7.57577300 & 0.91835500 & 2.27972700 \\
$\mathrm{C}$ & -5.53380900 & -1.88617200 & 1.90091700 \\
$\mathrm{H}$ & -5.90075800 & -2.19710900 & 0.91671600 \\
$\mathrm{H}$ & -6.03182500 & -2.51319500 & 2.65107700 \\
$\mathrm{H}$ & -4.45849500 & -2.09321300 & 1.93786100 \\
$\mathrm{C}$ & -5.36940500 & -0.07666800 & 3.61354100 \\
$\mathrm{H}$ & -5.57022100 & 0.96568300 & 3.88643600 \\
$\mathrm{H}$ & -4.30021200 & -0.26765500 & 3.75731300 \\
$\mathrm{H}$ & -5.91741100 & -0.71341300 & 4.31747500 \\
$\mathrm{C}$ & 5.26195100 & -0.53332600 & 2.83571700 \\
$\mathrm{C}$ & 4.70692900 & -1.91498900 & 3.25193900 \\
$\mathrm{H}$ & 3.61458700 & -1.93583000 & 3.16454000 \\
$\mathrm{H}$ & 4.96400800 & -2.13128800 & 4.29602600 \\
$\mathrm{H}$ & 5.1165300 & -2.72470300 & 2.63571300 \\
$\mathrm{C}$ & 4.64173100 & 0.52588700 & 3.76714400 \\
$\mathrm{H}$ & 3.55071900 & 0.56367700 & 3.67324100 \\
$\mathrm{H}$ & 5.03666100 & 1.52798400 & 3.56591700 \\
$\mathrm{H}$ & 4.87771400 & 0.28243300 & 4.80943800 \\
$\mathrm{C}$ & 6.79567400 & -0.51861500 & 3.04480200 \\
$\mathrm{H}$ & 7.29668400 & -1.29197900 & 2.45290200 \\
$\mathrm{H}$ & 7.03861100 & -0.69613000 & 4.09968400 \\
$\mathrm{H}$ & 7.22029800 & 0.44966200 & 2.75644100 \\
$\mathrm{C}$ & -5.63150800 & 2.28314700 & -3.25739800 \\
$\mathrm{H}$ & -4.89506300 & 3.08499600 & -3.14250900 \\
$\mathrm{H}$ & -6.53731400 & 2.58085700 & -2.71660600 \\
$\mathrm{H}$ & -5.88035300 & 2.20848800 & -4.32334500 \\
$\mathrm{H}$ & 2.04422900 & -3.29624500 & 2.17911700 \\
& & & \\
\hline & &
\end{tabular}


Enol (Table S7, entry 1)

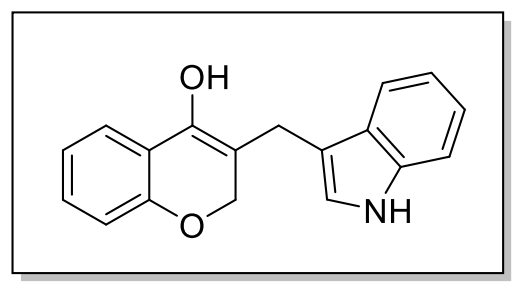

SCF energy [B3LYP-GD3BJ/6-311+G**/SMD (Chlorobenzene)]: -900.480994622 a.u. Thermal correction to Gibbs free energy at $258 \mathrm{~K}: 0.252285000$ a.u.

Gibbs free energy at $258 \mathrm{~K}$ [B3LYP-GD3BJ/6-311+G**/SMD (Chlorobenzene)]: -900.228709622 a.u.

\begin{tabular}{crrr} 
O 1 & & & \\
$\mathrm{C}$ & 1.69486400 & 1.92042700 & 0.81190600 \\
$\mathrm{C}$ & 3.61083000 & 0.68215500 & 0.13794100 \\
$\mathrm{C}$ & 2.80791100 & -0.39861400 & -0.27695400 \\
$\mathrm{C}$ & 1.37335200 & -0.15403300 & -0.44297200 \\
$\mathrm{C}$ & 0.80898600 & 0.97739300 & 0.03042800 \\
$\mathrm{H}$ & 5.58435200 & 1.39914200 & 0.55114700 \\
$\mathrm{H}$ & 1.66469700 & 1.70503200 & 1.89619100 \\
$\mathrm{C}$ & 4.99437800 & 0.54278100 & 0.23997600 \\
$\mathrm{C}$ & 3.42058100 & -1.62350200 & -0.57007200 \\
$\mathrm{C}$ & 4.80200800 & -1.77181200 & -0.45550100 \\
$\mathrm{C}$ & 5.58579600 & -0.68586500 & -0.05629800 \\
$\mathrm{H}$ & 2.79920500 & -2.44983400 & -0.89887000 \\
$\mathrm{H}$ & 5.26596000 & -2.72716400 & -0.68321100 \\
$\mathrm{H}$ & 6.66359200 & -0.79434100 & 0.03162400 \\
$\mathrm{O}$ & 0.70637000 & -1.13146200 & -1.12690800 \\
$\mathrm{O}$ & 3.06653100 & 1.90942100 & 0.39076700 \\
$\mathrm{C}$ & -0.64739600 & 1.34674200 & -0.17121200 \\
$\mathrm{H}$ & -0.84766300 & 2.29559900 & 0.34705000 \\
$\mathrm{H}$ & -0.82432900 & 1.56584100 & -1.23693400 \\
$\mathrm{C}$ & -1.66532200 & 0.32116000 & 0.27704300 \\
$\mathrm{C}$ & -3.06327800 & 0.30108300 & -0.09110500 \\
$\mathrm{C}$ & -1.48659600 & -0.71693600 & 1.16283800 \\
$\mathrm{C}$ & -3.67000900 & -0.77771000 & 0.60793200 \\
$\mathrm{~N}$ & -2.68437600 & -1.37401300 & 1.36876800 \\
$\mathrm{C}$ & -3.85727700 & 1.09065800 & -0.94100300 \\
$\mathrm{C}$ & -5.20633300 & 0.78872700 & -1.07669600 \\
$\mathrm{H}$ & -5.82910000 & 1.39185700 & -1.73150700 \\
$\mathrm{C}$ & -5.78484000 & -0.29029700 & -0.37705900 \\
$\mathrm{H}$ & -6.84280200 & -0.50259700 & -0.50394500 \\
$\mathrm{C}$ & -5.02738900 & -1.08753100 & 0.47301700 \\
& & & \\
\hline
\end{tabular}


$-1.48545300$

$-5.47468400$

$-1.91799500$

1. 01309700

$\mathrm{H}$

$-2.80141200 \quad-2.19908000$

1. 93597800

1. 36964700

2. 95707300

0. 67976000

$-0.58898500$

$-1.03289600$

1. 67608400

$\mathrm{H}$

$-0.24948900$

$-1.01044400$

$-0.97651900$ 
TS-S1 (Table S7, entry 2)

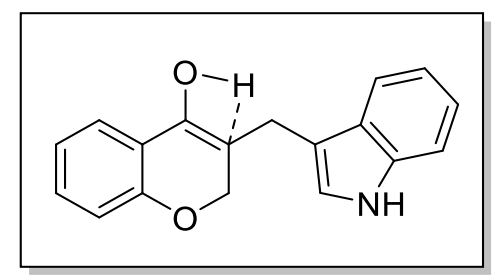

SCF energy [B3LYP-GD3BJ/6-311+G**/SMD (Chlorobenzene)]: -900.3887433930

a.u.

Thermal correction to Gibbs free energy at $258 \mathrm{~K}: 0.247148000$ a.u.

Gibbs free energy at $258 \mathrm{~K}$ [B3LYP-GD3BJ/6-311+G**/SMD (Chlorobenzene)]:

-900.141595393 a.u.

\begin{tabular}{crrr} 
O 1 & & & \\
H & -0.84150000 & -2.26562300 & -0.67140800 \\
C & -1.04554200 & -0.42557300 & 1.57521200 \\
C & -3.01485000 & 0.65871600 & 0.71925600 \\
C & -3.15692300 & -0.34525400 & -0.27974900 \\
C & -1.99400200 & -1.14840500 & -0.52321000 \\
C & -0.76084800 & -0.97536000 & 0.20149000 \\
H & -3.92440100 & 2.36689000 & 1.65006400 \\
H & -1.56229600 & -1.14652500 & 2.22189500 \\
C & -4.04429400 & 1.59625800 & 0.89577600 \\
C & -4.34784600 & -0.43044900 & -1.03034300 \\
C & -5.36273600 & 0.48977100 & -0.83510100 \\
C & -5.19701200 & 1.50398200 & 0.12654900 \\
H & -4.43241200 & -1.21786600 & -1.77319800 \\
H & -6.27751500 & 0.43403400 & -1.41668800 \\
H & -5.99382900 & 2.22645200 & 0.28373500 \\
O & -1.88339500 & -2.12250500 & -1.36560600 \\
O & -1.93250100 & 0.75702100 & 1.51279600 \\
C & 0.33606600 & -0.20718100 & -0.57284400 \\
H & 0.17841200 & 0.88025500 & -0.47551600 \\
H & 0.24030300 & -0.42466100 & -1.64586700 \\
C & 1.72830500 & -0.53694300 & -0.10447100 \\
C & 2.90842600 & 0.27343400 & -0.29473200 \\
C & 2.13979100 & -1.66488200 & 0.56023900 \\
C & 4.00395300 & -0.42998700 & 0.27958600 \\
N & 3.50419400 & -1.61048000 & 0.78885600 \\
C & 3.14732300 & 1.52238700 & -0.89440400 \\
C & 4.43924900 & 2.03358400 & -0.90302700 \\
H & 4.63282400 & 2.99888200 & -1.36309300 \\
C & 5.50842600 & 1.31884300 & -0.32499500 \\
& & & \\
\hline
\end{tabular}


1. 74299400

$-0.34744400$

C

5. 30642200

0.07977600

0.27265000

$\mathrm{H}$

2. $33175000 \quad 2.08185600$

$-1.34561400$

$\mathrm{H}$

6. 13161000

$-0.47127200$

0.71703600

$\mathrm{H}$

4. 03860500

$-2.30683000$

1. 28364000

$\mathrm{H}$

$-0.15185800$

$-0.06537700$

2. 08602600

H

1. 55599400

$-2.51227200$

0. 88963000 
TS-S2 (Table S7, entry 3)

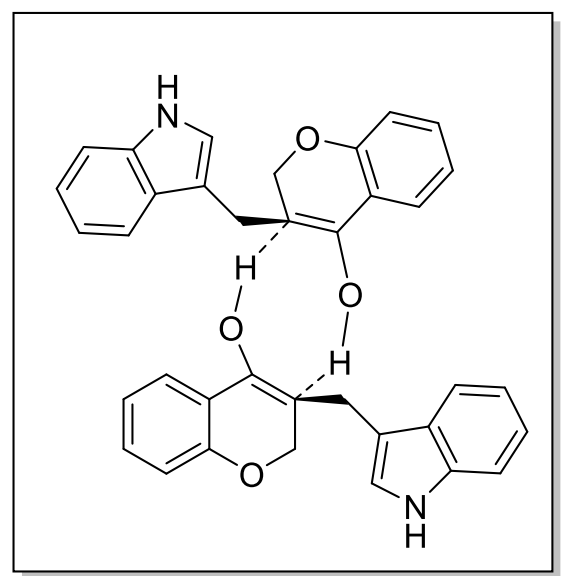

SCF energy [B3LYP-GD3BJ/6-311+G**/SMD (Chlorobenzene)]: -1800.9315224500 a.u.

Thermal correction to Gibbs free energy at $258 \mathrm{~K}: 0.518285000$ a.u.

Gibbs free energy at $258 \mathrm{~K}$ [B3LYP-GD3BJ/6-311+G**/SMD (Chlorobenzene)]:

-1800.413237450 a.u.

01

$\begin{array}{rrrr}\text { H } & 0.17662600 & -1.11628700 & -1.09139000 \\ \mathrm{H} & -0.44849000 & -0.68176100 & 1.11866500 \\ \mathrm{C} & 2.84764700 & -1.75436100 & -1.33607700 \\ \mathrm{C} & 2.73304800 & -3.60048000 & 0.12144500 \\ \mathrm{C} & 1.68112300 & -2.98701700 & 0.83145900 \\ \mathrm{C} & 1.49604800 & -1.51553700 & 0.72068700 \\ \mathrm{C} & 2.11876300 & -0.87983600 & -0.33615800 \\ \mathrm{H} & 3.85761300 & -5.38745500 & -0.24320200 \\ \mathrm{H} & 2.17203000 & -2.16687800 & -2.10705600 \\ \mathrm{C} & 3.03309400 & -4.95072200 & 0.31207900 \\ \mathrm{C} & 0.93635400 & -3.76399500 & 1.72697200 \\ \mathrm{C} & 1.21218800 & -5.11816600 & 1.90460900 \\ \mathrm{C} & 2.26650600 & -5.70666900 & 1.19871900 \\ \mathrm{H} & 0.15881600 & -3.26950400 & 2.30093300 \\ \mathrm{H} & 0.62218600 & -5.70949500 & 2.59945700 \\ \mathrm{H} & 2.49381300 & -6.76078200 & 1.33654900 \\ \mathrm{O} & 0.76393100 & -0.92071000 & 1.64223100 \\ \mathrm{O} & 3.52088400 & -2.87807900 & -0.7304100 \\ \mathrm{C} & 2.33807400 & 0.61865300 & -0.37345300 \\ \mathrm{H} & 1.70252400 & 1.06873900 & 0.39779000 \\ \mathrm{H} & 2.00965800 & 1.05224200 & -1.33325400 \\ \mathrm{C} & 3.77365100 & 1.03572500 & -0.14545200 \\ \mathrm{C} & 4.33222800 & 2.34092500 & -0.40835700 \\ \mathrm{C} & 4.79169800 & 0.27999900 & 0.37931600 \\ & & & \end{array}$


$-0.01627200$

5. 94909700

1. 03560300

0. 46192600

3. 81248300

3. 53665600

$-0.93376700$

4. 64619000

4. 64144500

$-1.05996900$

$\mathrm{H}$

4. 25209700

5. 56963600

$-1.46543000$

5. 99864700

4. 58036500

$-0.66675600$

$\mathrm{H}$

6. 62730700

5. 46015900

$-0.77552500$

6. 54286500

3. 41389600

-0. 14014000

2. 76985300

3. 59848900

$-1.23605600$

7. 58558500

3. 36797600

0. 16463100

6. 83799600

0. 69874500

0. 79601800

3. 63381000

$-1.19367800$

$-1.84611000$

4. 78392300

$-0.75439800$

0. 69331200

$-2.66813600$

$-0.54796200$

1. 52893100

$-4.03638200$

$-1.60688300$

$-0.07589600$

$-2.87963800$

$-2.01038200$

$-0.79846900$

$-1.66456800$

$-1.28603700$

$-0.56485400$

$-1.53946800$

$-0.36408800$

0. 52398200

$-6.16468800$

$-1.78451400$

0. 11967800

$-2.48398100$

$-1.43669700$

2. 14659000

-5. 29242100

$-2.12012800$

$-0.43100400$

$-3.00580100$

$-2.97963500$

$-1.82261700$

$-4.24291000$

$-3.50562200$

-2. 14091400

$-5.38489800$

$-3.06161500$

$-1.44591200$

$-2.11159500$

$-3.28881600$

$-2.35283600$

$-4.33746900$

$-4.25299100$

$-2.92215400$

$-6.35884900$

-3. 47253300

$-1.69822700$

$-0.69419400$

$-1.47066300$

$-1.44480400$

$-3.97954400$

$-0.72385700$

0. 93123200

$-1.20803600$

1. 11040800

0. 11154000

$-0.45706200$

1. 08818500

$-0.68368200$

$-0.71708400$

1. 58340400

0. 96791300

$-2.39379500$

1. 92968600

$-0.31491100$

$-3.15188000$

2. 84583000

0. 50513800

$-2.97839800$

1. 95384500

$-1.55878200$

$-4.18602700$

3. 38572700

$-0.30827100$

$-4.05519400$

2. 81861600

$-1.55943400$

$-3.05140700$

3. 27483100

1. 84107000

$-3.96550800$

4. 20167100

2. 32521700

$-3.89353500$

4. 53947200

3. 35529200

$-4.98621900$

4. 71725100

1. 49997200

$-5.68662500$

5. 44163100

1. 90635300

$-5.10917200$

4. 31936200

0. 17410100

$-2.26280700$

2. 89520700

2. 48659500 
$-0.46342700$

2. 18148300

$-2.70686800$

1. 41916100

$-2.45941200$ 
TS-H 2 O (Table S7, entry 4)

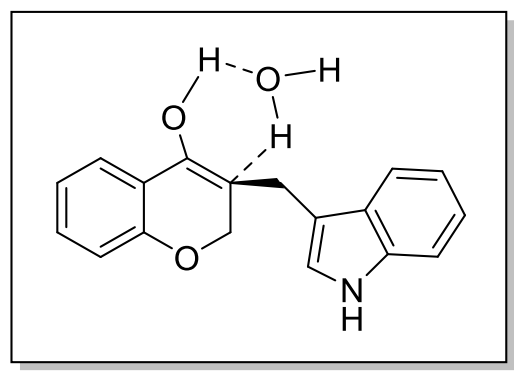

SCF energy [B3LYP-GD3BJ/6-311+G**/SMD (Chlorobenzene)]: -976.9046430940 a.u.

Thermal correction to Gibbs free energy at $258 \mathrm{~K}: 0.270197000$ a.u.

Gibbs free energy at $258 \mathrm{~K}$ [B3LYP-GD3BJ/6-311+G**/SMD (Chlorobenzene)]: -976.634446094 a.u.

\begin{tabular}{crrr} 
O 1 & & & \\
$\mathrm{H}$ & 1.63742500 & 3.00602100 & -0.86017300 \\
$\mathrm{O}$ & 1.15833700 & 3.79687900 & 0.02229400 \\
$\mathrm{H}$ & 0.99930400 & 2.72449300 & 0.63891000 \\
$\mathrm{H}$ & 1.91326200 & 4.25897200 & 0.42479900 \\
$\mathrm{C}$ & 0.84656900 & 0.45191300 & 1.79711100 \\
$\mathrm{C}$ & 2.42251400 & -0.99126900 & 0.76457700 \\
$\mathrm{C}$ & 2.78568700 & 0.03217300 & -0.14364000 \\
$\mathrm{C}$ & 1.86094500 & 1.14169100 & -0.33511300 \\
$\mathrm{C}$ & 0.75569700 & 1.31763100 & 0.55552500 \\
$\mathrm{H}$ & 2.88930200 & -2.93677200 & 1.53759500 \\
$\mathrm{H}$ & 1.53021700 & 0.88495800 & 2.54121900 \\
$\mathrm{C}$ & 3.19179400 & -2.15980100 & 0.84291200 \\
$\mathrm{C}$ & 3.94816100 & -0.11811600 & -0.92299700 \\
$\mathrm{C}$ & 4.72231200 & -1.26441200 & -0.82402200 \\
$\mathrm{C}$ & 4.33177000 & -2.28594600 & 0.05671600 \\
$\mathrm{H}$ & 4.20549400 & 0.67904600 & -1.61294900 \\
$\mathrm{H}$ & 5.61860700 & -1.37589900 & -1.42647200 \\
$\mathrm{H}$ & 4.93300000 & -3.18796600 & 0.13668600 \\
O & 2.09254800 & 2.00144400 & -1.27921500 \\
O & 1.32821000 & -0.90042500 & 1.55168400 \\
$\mathrm{C}$ & -0.65437000 & 1.52606500 & -0.05939600 \\
$\mathrm{H}$ & -0.53894600 & 2.08940600 & -0.99292200 \\
$\mathrm{H}$ & -1.23451700 & 2.18269400 & 0.60047100 \\
$\mathrm{C}$ & -1.45072300 & 0.26801500 & -0.29654400 \\
$\mathrm{C}$ & -2.87738200 & 0.12674700 & -0.11443900 \\
$\mathrm{C}$ & -1.00463600 & -0.94230400 & -0.76850900 \\
$\mathrm{C}$ & -3.22633300 & -1.19874600 & -0.49580600 \\
$\mathrm{~N}$ & -2.06294800 & -1.82195100 & -0.89647400 \\
& & & \\
\hline & & & \\
& & &
\end{tabular}




$\begin{array}{lrrr}\mathrm{C} & -3.89524700 & 0.98880200 & 0.33108100 \\ \mathrm{C} & -5.20143000 & 0.51984800 & 0.39412900 \\ \mathrm{H} & -5.99314300 & 1.17987500 & 0.73831700 \\ \mathrm{C} & -5.52100600 & -0.80010200 & 0.01481200 \\ \mathrm{H} & -6.55193900 & -1.13850200 & 0.07342800 \\ \mathrm{C} & -4.53969400 & -1.67581900 & -0.43531300 \\ \mathrm{H} & -3.66605500 & 2.01111800 & 0.62112200 \\ \mathrm{H} & -4.78550600 & -2.69279900 & -0.73083500 \\ \mathrm{H} & -1.98298000 & -2.78610000 & -1.17878300 \\ \mathrm{H} & -0.13036400 & 0.31262800 & 2.26217900 \\ \mathrm{H} & -0.00305100 & -1.25892800 & -1.02187700\end{array}$

$\mathbf{H}_{\mathbf{2}} \mathbf{O}$ (Table S7, entry 5)

$\mathrm{H}_{2} \mathrm{O}$

SCF energy [B3LYP-GD3BJ/6-311+G**/SMD (Chlorobenzene)]: -76.4651401167 a.u. Thermal correction to Gibbs free energy at $258 \mathrm{~K}: 0.006353000$ a.u.

Gibbs free energy at $258 \mathrm{~K}$ [B3LYP-GD3BJ/6-311+G**/SMD (Chlorobenzene)]: -76.4587871170 a.u.

$\begin{array}{crrr}01 & & & \\ 0 & 0.00000000 & 0.00000000 & 0.11972000 \\ H & 0.00000000 & -0.76156000 & -0.47887900 \\ H & 0.00000000 & 0.76156000 & -0.47887900\end{array}$


2b (Table S8, entry 1)

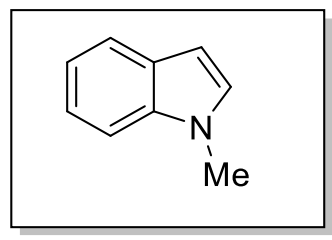

SCF energy [B3LYP-GD3BJ/6-311+G**/SMD (Chlorobenzene)]: -403.284380513 a.u. Thermal correction to Gibbs free energy at $258 \mathrm{~K}: 0.130534000$ a.u.

Gibbs free energy at $258 \mathrm{~K}$ [B3LYP-GD3BJ/6-311+G**/SMD (Chlorobenzene)]: -403.153846513 a.u.

01

$\begin{array}{lrrr}\text { C } & -0.38926200 & 0.98539100 & 0.00000000 \\ \mathrm{C} & 0.15176200 & -0.33251500 & -0.00000100 \\ \mathrm{C} & -0.65970700 & -1.47318500 & 0.00000000 \\ \mathrm{C} & -2.03698700 & -1.28158300 & 0.00000100 \\ \mathrm{C} & -2.59445800 & 0.01373300 & 0.00000100 \\ \mathrm{C} & -1.78585100 & 1.14329700 & 0.00000100 \\ \mathrm{C} & 0.72610400 & 1.88906800 & -0.00000200 \\ \mathrm{C} & 1.86016100 & 1.12104000 & 0.00000100 \\ \mathrm{H} & -0.23439100 & -2.47306100 & 0.00000000 \\ \mathrm{H} & -2.69540500 & -2.14607500 & 0.00000100 \\ \mathrm{H} & -3.67533100 & 0.12628000 & 0.00000100 \\ \mathrm{H} & -2.22543600 & 2.13768100 & 0.00000100 \\ \mathrm{H} & 0.69013900 & 2.96990200 & -0.00000300 \\ \mathrm{H} & 2.90076300 & 1.41741100 & 0.00000100 \\ \mathrm{~N} & 1.53003800 & -0.22387900 & -0.00000200 \\ \mathrm{C} & 2.46033200 & -1.33261300 & 0.00000000 \\ \mathrm{H} & 2.32820400 & -1.95943900 & 0.89044000 \\ \mathrm{H} & 2.32818400 & -1.95945600 & -0.89042500 \\ \mathrm{H} & 3.48044800 & -0.94188700 & -0.00001500\end{array}$


INT-I'-Me (Table S8, entry 2)

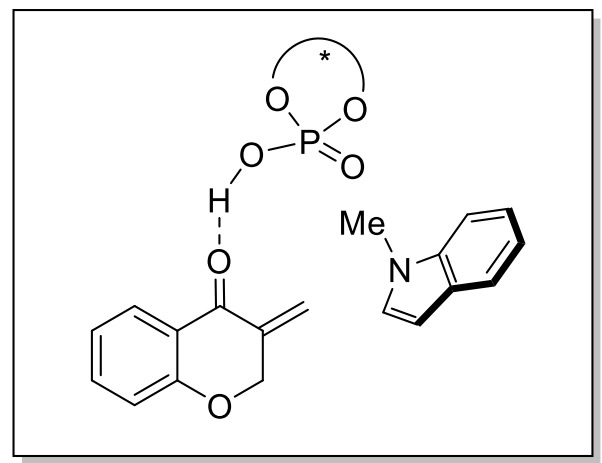

SCF energy [B3LYP-GD3BJ/6-311+G**/SMD (Chlorobenzene)]: -3330.998809810 a.u.

Thermal correction to Gibbs free energy at $258 \mathrm{~K}: 1.133465000$ a.u.

Gibbs free energy at $258 \mathrm{~K}$ [B3LYP-GD3BJ/6-311+G**/SMD (Chlorobenzene)]: -3329.865344810 a.u.

01

P

0

0

0

0

C

C

C

C

C

C

C

C

C

C

C

C

C

C

C

C

C

$\mathrm{H}$

$\mathrm{H}$

$\mathrm{H}$

H
0.65539200

1. 94238400

$-0.00827300$

$-0.32549700$

0. 94839200

2. 99398200

4. 09640500

5. 06581300

4. 93887000

3. 85405100

3. 49112600

2. 02841800

1. 94360500

2. 90214100

2. 48744400

1. 29450800

0. 10693000

$-1.21528600$

-2. 15321000

$-1.79462100$

$-0.43799600$

0. 49615300

5. 93734900

5. 68768100

4. 13730500

3. 59655400
$-1.48023900$

$-1.93926700$

$-2.87460000$

$-0.97244100$

$-0.59475200$

$-2.57877800$

$-1.83420100$

$-2.53032600$

$-3.88393600$

$-4.59245400$

-6. 04750800

$-6.09704800$

$-4.98591200$

$-3.95280100$

$-5.54734200$

-6. 30920100

$-5.53963100$

$-5.57374500$

-4. 75949200

$-3.85585600$

$-3.82227500$

$-4.69590800$

$-1.98527700$

-4. 37607100

-6. 70664400

-6. 36603100
0. 08673300

$-0.80882100$

0. 59051700

$-1.04732900$

1. 23391700

$-0.13735400$

0. 33200200

1. 07735400

1. 38277300

0. 87854700

1. 07984000

0. 58684300

$-0.51302700$

0. 07062400

$-1.87075800$

$-2.48579500$

$-1.95143100$

$-2.37906700$

$-1.74719000$

$-0.73143700$

$-0.34849700$

$-0.89899900$

1. 42609400

1. 99844500

0. 48285900

2. 12328500 


\begin{tabular}{|c|c|c|c|}
\hline $\mathrm{H}$ & 1. 72857800 & -7.08004800 & 0.21021700 \\
\hline $\mathrm{H}$ & 1. 35148100 & -5.83296300 & 1. 40723800 \\
\hline $\mathrm{H}$ & 3. 37960400 & -6.16733600 & -1.73780200 \\
\hline $\mathrm{H}$ & 2. 76043100 & -4.70406100 & -2.51529200 \\
\hline $\mathrm{H}$ & 1. 27364500 & -7.35649400 & -2.15247800 \\
\hline $\mathrm{H}$ & 1. 32436100 & -6.32885400 & -3.58126400 \\
\hline $\mathrm{H}$ & -1.52118100 & -6.23189300 & -3.18855700 \\
\hline H & -3.19635800 & -4.81417100 & -2.04321400 \\
\hline C & -2.85028400 & -3.01439200 & -0.10331700 \\
\hline $\mathrm{C}$ & -3.84940600 & -2.43940600 & -0.90336000 \\
\hline $\mathrm{C}$ & -2.92289600 & -2.86148900 & 1. 28925100 \\
\hline C & -4.92686700 & -1.74466600 & -0.34257200 \\
\hline C & -3.98058900 & -2.17007300 & 1. 89069800 \\
\hline C & -4.96991800 & -1.63145000 & 1. 05461600 \\
\hline $\mathrm{C}$ & 4. 28652500 & -0.38313700 & 0.06000400 \\
\hline C & 3. 98577400 & 0.16834600 & -1.19853400 \\
\hline C & 4. 84983200 & 0.44393500 & 1. 03750700 \\
\hline C & 4. 23532800 & 1.51134900 & -1.48159500 \\
\hline $\mathrm{C}$ & 5. 13540200 & 1. 79537300 & 0.78931500 \\
\hline $\mathrm{C}$ & 4. 81466300 & 2. 30415400 & -0.47297100 \\
\hline $\mathrm{H}$ & -5.80711700 & -1.10906900 & 1. 50814400 \\
\hline $\mathrm{H}$ & -3.76403000 & -2.53762900 & -1.97898800 \\
\hline $\mathrm{H}$ & -2.14746200 & -3.30758700 & 1. 89781900 \\
\hline $\mathrm{H}$ & 5. 02300400 & 3. 34542900 & -0.68994300 \\
\hline $\mathrm{H}$ & 5. 05950700 & 0.02316900 & 2. 01574700 \\
\hline $\mathrm{H}$ & 3. 56172400 & -0.47730900 & -1.95507800 \\
\hline C & -6.06080000 & -1.14239200 & -1.19669300 \\
\hline $\mathrm{C}$ & -7.38451900 & -1.87154100 & -0.86223200 \\
\hline $\mathrm{H}$ & -8.21098400 & -1.45658800 & -1.45263900 \\
\hline $\mathrm{H}$ & -7.64629800 & -1.77202700 & 0.19651900 \\
\hline $\mathrm{H}$ & -7.30911700 & -2.94150600 & -1.08787100 \\
\hline C & -6.21811800 & 0.36489900 & -0.88748000 \\
\hline $\mathrm{H}$ & -7.02656700 & 0.79446100 & -1.49168900 \\
\hline $\mathrm{H}$ & -5.29640600 & 0.91184600 & -1.11467800 \\
\hline $\mathrm{H}$ & -6.46305900 & 0.54375700 & 0.16486800 \\
\hline $\mathrm{C}$ & -5.79982600 & -1.29116000 & -2.70782200 \\
\hline $\mathrm{H}$ & -5.73735000 & -2.34238700 & -3.01087100 \\
\hline $\mathrm{H}$ & -4.87283200 & -0.79201500 & -3.01192100 \\
\hline $\mathrm{H}$ & -6.62254500 & -0.83571700 & -3.27062600 \\
\hline C & 3. 91598100 & 2. 13831000 & -2.85318700 \\
\hline C & 2. 92421700 & 3. 31077900 & -2.66583100 \\
\hline $\mathrm{H}$ & 1. 98087700 & 2. 95449800 & -2.2366120 \\
\hline $\mathrm{H}$ & 2. 70195700 & 3. 78061900 & -3.63230900 \\
\hline & 3. 32570300 & 4. 08700800 & -2.005942 \\
\hline
\end{tabular}


3. 27392800

2. 33039000

3. 93985500

3. 05835800

5. 81355800

7. 21997000

7. 72885300

7. 84104600

7. 16480200

5. 98109500

6. 61853300

6. 45264000

5. 01675500

4. 98019500

4. 84744800

3. 98396200

5. 47965300

-4. 10589000

$-5.36577000$

-6. 27748100

$-5.47781200$

-5. 29722100

-4. 23781500

$-5.12302200$

$-3.35854300$

$-4.32919200$

$-2.88365800$

$-2.76976400$

$-3.00410800$

$-1.95413800$

5. 22025500

5. 93875400

5. 70389800

5. 00893000

$-0.35484700$

$-3.11054400$

$-2.87002800$

$-1.76794900$

$-1.52617500$

$-2.43798100$

$-4.03633700$

$-2.39066400$

$-3.18198000$

$-0.98507900$
1. 12947300

0. 73187400

0. 28540100

1. 62494300

2. 64329700

2. 06114300

2. 64775700

2. 07777500

1. 02410700

4. 11736600

4. 22416000

4. 67892400

4. 59274400

2. 60549400

1. 58607800

3. 03460200

3. 18583200

$-2.02129100$

$-2.77958000$

$-2.38966100$

$-2.68485000$

$-3.84614600$

$-0.52630800$

$-0.06562500$

0. 04107400

$-0.41476200$

$-2.59048700$

$-3.66803300$

$-2.43384300$

$-2.09873300$

2. 66616600

1. 85245400

3. 42669600

3. 11940000

0. 01256700

3. 77884400

4. 33641900

3. 46019900

2. 41665800

2. 45179800

5. 89147100

4. 50764200

5. 23456600

3. 52083300
$-3.82499800$

$-3.43462400$

$-4.03708600$

$-4.77892500$

1. 88448300

2. 16792200

2. 94293200

1. 26495500

2. 51597900

1. 46964000

0. 58459200

2. 28401500

1. 25586900

3. 18686700

3. 56245300

3. 03286200

3. 97222900

3. 42019600

3. 90226500

3. 43666400

4. 98953600

3. 65928400

3. 79483400

3. 34320200

3. 46932000

4. 88243300

4. 16598300

4. 00258800

5. 24405700

3. 85841400

$-3.49641600$

$-3.64785300$

$-2.87427200$

$-4.47302900$

$-1.21061400$

$-0.25153800$

$-2.53926000$

$-2.67281900$

$-1.66456600$

$-0.49041000$

$-3.44403300$

0. 14589000

$-3.56998000$

$-3.84309500$ 
-1. 28223000

-2. 39103500

$-0.15392300$

$-0.67159900$

$-2.63381100$

$-0.64424900$

$-3.67900500$

$-2.61868000$

$-3.30379000$

$-2.09252700$

$-3.93719700$

$-1.46742300$

-0. 45794700

$-0.28167100$

$-1.13679300$

-2. 14154700

$-2.31052100$

$-1.35273200$

$-0.32388500$

0. 49583400

$-1.02463000$

$-2.79013700$

$-3.08585700$

$-1.94501500$

0. 07028600

0. 22586700

1. 31065800

0. 97226000

2. 14363000

1. 65438000
4. 42187200

5. 27399600

2. 82929800

4. 46485100

5. 97957400

1. 55872200

4. 33844400

1. 38610700

1. 41844000

0. 45280200

3. 69240100

3. 82471000

4. 09285200

5. 36438400

6. 37981800

6. 13627500

4. 87455800

2. 43371100

1. 92338300

5. 55533500

7. 37804200

6. 95210200

4. 70025700

1. 88420900

0. 91869200

2. 91808100

2. 74719200

2. 96205400

3. 41690900

1. 71340700
$-4.85119400$

$-4.70995000$

$-3.93169100$

$-5.74776400$

$-5.50017500$

$-1.82865000$

$-1.45441800$

0. 30139300

1. 14224500

0. 13706200

0. 45457200

3. 38736500

2. 41504600

1. 85434500

2. 27431200

3. 23379600

3. 79229400

3. 71991100

2. 96517900

1. 11952400

1. 85939200

3. 54210900

4. 53473100

4. 43867900

2. 89341800

2. 17940500

1. 23006200

0. 21004900

1. 47039000

1. 26726200 
INT-I-Me (Table S8, entry 3)

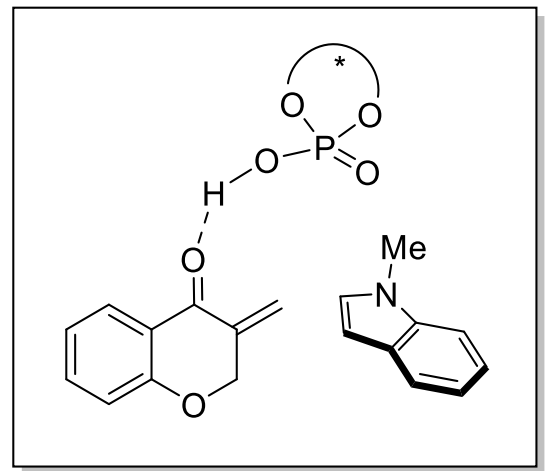

SCF energy [B3LYP-GD3BJ/6-311+G**/SMD (Chlorobenzene)]: -3330.997669430 a.u.

Thermal correction to Gibbs free energy at $258 \mathrm{~K}: 1.132585000$ a.u.

Gibbs free energy at $258 \mathrm{~K}$ [B3LYP-GD3BJ/6-311+G**/SMD (Chlorobenzene)]: -3329.865084430 a.u.

\begin{tabular}{lrrr}
01 & & & \\
$\mathrm{P}$ & -0.49986000 & -1.47971600 & -0.07076500 \\
0 & 0.19726500 & -2.72031300 & -0.87408600 \\
0 & -1.86739100 & -2.12302100 & 0.53773700 \\
0 & -0.99268000 & -0.59248800 & -1.28645800 \\
0 & 0.30607800 & -0.85220800 & 0.99639100 \\
$\mathrm{C}$ & 0.61829900 & -3.82239700 & -0.11910200 \\
$\mathrm{C}$ & 1.94145800 & -3.88885900 & 0.36358300 \\
$\mathrm{C}$ & 2.27020700 & -4.98238400 & 1.18474800 \\
$\mathrm{C}$ & 1.33232000 & -5.94802600 & 1.54561100 \\
$\mathrm{C}$ & 0.04296400 & -5.86439700 & 1.03163200 \\
$\mathrm{C}$ & -1.14462200 & -6.76468600 & 1.29199500 \\
$\mathrm{C}$ & -2.32362900 & -5.91903600 & 0.76400700 \\
$\mathrm{C}$ & -1.71014500 & -5.06100800 & -0.39428700 \\
$\mathrm{C}$ & -0.30823200 & -4.82447200 & 0.15779800 \\
$\mathrm{C}$ & -1.66919700 & -5.90757000 & -1.71092700 \\
$\mathrm{C}$ & -3.09197100 & -5.78556900 & -2.29807700 \\
$\mathrm{C}$ & -3.52531000 & -4.41611900 & -1.82198400 \\
$\mathrm{C}$ & -4.59037200 & -3.63792000 & -2.26123700 \\
$\mathrm{C}$ & -4.79556200 & -2.38360000 & -1.68826800 \\
$\mathrm{C}$ & -3.91998500 & -1.85234400 & -0.72597400 \\
$\mathrm{C}$ & -2.82676800 & -2.65191700 & -0.33359200 \\
$\mathrm{C}$ & -2.66384100 & -3.94744300 & -0.81736600 \\
$\mathrm{H}$ & 3.28975000 & -5.06360100 & 1.54916200 \\
$\mathrm{H}$ & 1.61298300 & -6.75712900 & 2.21547800 \\
$\mathrm{H}$ & -1.05541000 & -7.71311900 & 0.74317500 \\
$\mathrm{H}$ & -1.25186700 & -7.02413300 & 2.35153200 \\
$\mathrm{H}$ & -3.17691700 & -6.51905800 & 0.43232100 \\
& & & \\
\hline & & &
\end{tabular}




\begin{tabular}{|c|c|c|c|}
\hline $\mathrm{H}$ & -2.67667200 & -5.24425700 & 1. 55214700 \\
\hline $\mathrm{H}$ & -1.35862400 & -6.94187000 & -1.53234400 \\
\hline $\mathrm{H}$ & -0.94496100 & -5.45456500 & -2.39736700 \\
\hline $\mathrm{H}$ & -3.75436800 & -6.57179000 & -1.90868400 \\
\hline $\mathrm{H}$ & -3.10784700 & -5.87446700 & -3.39046700 \\
\hline $\mathrm{H}$ & -5.26299700 & -4.00131100 & -3.03427200 \\
\hline H & -5.65178100 & -1.78840800 & -1.99159500 \\
\hline C & -4.19074200 & -0.50510100 & -0.15324400 \\
\hline $\mathrm{C}$ & -4.60821000 & 0.54104800 & -0.98939700 \\
\hline $\mathrm{C}$ & -4.12026300 & -0.28299400 & 1. 23014100 \\
\hline C & -4.97574000 & 1. 78894500 & -0.47246800 \\
\hline $\mathrm{C}$ & -4.47567600 & 0.94958600 & 1. 78800900 \\
\hline $\mathrm{C}$ & -4.90550400 & 1. 96241300 & 0.91745900 \\
\hline C & 2. 98168000 & -2.87572800 & 0.03876700 \\
\hline C & 3. 12481600 & -2.37265800 & -1.26611800 \\
\hline $\mathrm{C}$ & 3. 87892200 & -2.45713900 & 1. 02555300 \\
\hline $\mathrm{C}$ & 4. 13874300 & -1.46943000 & -1.58591900 \\
\hline $\mathrm{C}$ & 4. 91640500 & -1.55515900 & 0.74610900 \\
\hline $\mathrm{C}$ & 5. 02255100 & -1.07761700 & -0.56340200 \\
\hline $\mathrm{H}$ & -5.19976600 & 2. 91969600 & 1. 33799900 \\
\hline $\mathrm{H}$ & -4.62949800 & 0.36410900 & -2.05816600 \\
\hline $\mathrm{H}$ & -3.79700600 & -1.09627700 & 1. 86586900 \\
\hline H & 5.81327000 & -0.37735600 & -0.80558300 \\
\hline $\mathrm{H}$ & 3. 74785800 & -2.83007300 & 2. 03632500 \\
\hline $\mathrm{H}$ & 2. 43151600 & -2.70725400 & -2.02596400 \\
\hline $\mathrm{C}$ & -5.47062100 & 2. 94459200 & -1.36534500 \\
\hline C & -6.94414800 & 3. 25957100 & -1.01247100 \\
\hline $\mathrm{H}$ & -7.31935300 & 4. 08365800 & -1.63211100 \\
\hline $\mathrm{H}$ & -7.05634000 & 3. 55198800 & 0.03704400 \\
\hline $\mathrm{H}$ & -7.58300400 & 2. 38586700 & -1.18529700 \\
\hline C & -4.60887900 & 4. 20663800 & -1.12625900 \\
\hline $\mathrm{H}$ & -4.95287600 & 5. 02900100 & -1.76562100 \\
\hline $\mathrm{H}$ & -3.55535000 & 4. 01351200 & -1.35701900 \\
\hline $\mathrm{H}$ & -4.66576700 & 4. 55108400 & -0.08814200 \\
\hline $\mathrm{C}$ & -5.39957600 & 2. 59987800 & -2.86546200 \\
\hline $\mathrm{H}$ & -6.03470900 & 1. 74397700 & -3.12009500 \\
\hline $\mathrm{H}$ & -4.37583200 & 2. 37263100 & -3.18354200 \\
\hline $\mathrm{H}$ & -5.74881300 & 3. 45437200 & -3.45622100 \\
\hline $\mathrm{C}$ & 4. 32256800 & -0.90416100 & -3.00766600 \\
\hline $\mathrm{C}$ & 4. 21050400 & 0.63832700 & -2.96662300 \\
\hline $\mathrm{H}$ & 3. 22711300 & 0.94516400 & -2.59356900 \\
\hline $\mathrm{H}$ & 4. 34184200 & 1. 05839100 & -3.97201700 \\
\hline $\mathrm{H}$ & 4. 97285400 & 1. 08699100 & -2.32054000 \\
\hline $\mathrm{C}$ & 3. 25809100 & -1.42795400 & $-3.990949 c$ \\
\hline
\end{tabular}




\begin{tabular}{|c|c|c|c|}
\hline $\mathrm{H}$ & 2. 24458100 & -1.15339600 & -3.67798800 \\
\hline $\mathrm{H}$ & 3. 30047600 & -2.51797900 & $-4.0967930 c$ \\
\hline $\mathrm{H}$ & 3. 42953100 & -0.99485700 & -4.98340200 \\
\hline $\mathrm{C}$ & 5. 90046700 & -1.15344500 & 1. 86276200 \\
\hline $\mathrm{C}$ & 6. 71983900 & -2.39933800 & 2.27761100 \\
\hline $\mathrm{H}$ & 7. 42717800 & -2.14446900 & 3. 07692400 \\
\hline $\mathrm{H}$ & 7. 29180300 & -2.79376400 & 1. 42955700 \\
\hline $\mathrm{H}$ & 6.07262100 & -3.20262400 & 2.64647800 \\
\hline $\mathrm{C}$ & 6. 88400400 & -0.05595600 & 1. 41360100 \\
\hline $\mathrm{H}$ & 7. 51779700 & -0.38440200 & 0.58167100 \\
\hline $\mathrm{H}$ & 7. 54743200 & 0.20556700 & 2. 24598400 \\
\hline $\mathrm{H}$ & 6. 35766600 & 0.85591500 & 1. 10967800 \\
\hline $\mathrm{C}$ & 5. 12658100 & -0.61973000 & 3. 09097200 \\
\hline $\mathrm{H}$ & 4. 44778300 & -1.37011600 & 3.50876700 \\
\hline $\mathrm{H}$ & 4. 53051600 & 0.26008700 & 2.82852100 \\
\hline $\mathrm{H}$ & 5. 83031100 & -0.33467900 & 3. 88312500 \\
\hline $\mathrm{C}$ & -4.43534200 & 1. 21158200 & 3. 30700800 \\
\hline $\mathrm{C}$ & -5.86270600 & 1. 53716000 & 3. 80839100 \\
\hline $\mathrm{H}$ & -6.27573100 & 2. 42500900 & 3. 31749400 \\
\hline $\mathrm{H}$ & -5.85440400 & 1. 72615400 & 4. 88910800 \\
\hline $\mathrm{H}$ & -6.54513900 & 0.70171400 & 3. 61479300 \\
\hline $\mathrm{C}$ & -3.50419300 & 2. 41017200 & 3. 60758800 \\
\hline $\mathrm{H}$ & -3.83458900 & 3. 32275900 & 3. 09947800 \\
\hline $\mathrm{H}$ & -2.47508100 & 2. 19969700 & 3. 29357200 \\
\hline $\mathrm{H}$ & -3.49231200 & 2. 61941500 & 4. 68496000 \\
\hline $\mathrm{C}$ & -3.91354400 & -0.00302600 & 4. 09879500 \\
\hline $\mathrm{H}$ & -4.56197600 & -0.87818300 & 3. 97846500 \\
\hline $\mathrm{H}$ & -3.88476300 & 0.23945800 & 5. 16746600 \\
\hline $\mathrm{H}$ & -2.89982700 & -0.28472900 & 3.79344000 \\
\hline $\mathrm{C}$ & 5.71596900 & -1.30413000 & -3.54765100 \\
\hline $\mathrm{H}$ & 5. 81730700 & -2.39439200 & $-3.5981000 c$ \\
\hline $\mathrm{H}$ & 6. 52393300 & -0.92345000 & -2.91392900 \\
\hline $\mathrm{H}$ & 5. 86595000 & -0.90161600 & $-4.5574730 c$ \\
\hline $\mathrm{H}$ & -0.42392800 & 0.20023000 & -1.51001200 \\
\hline $\mathrm{C}$ & 0.30445000 & 4. 61050500 & -0.16196900 \\
\hline $\mathrm{C}$ & 0.92063500 & 5. 12138600 & -2.39319300 \\
\hline $\mathrm{C}$ & 0.98349000 & 3. 74892000 & $-2.7264530 c$ \\
\hline $\mathrm{C}$ & 0.35832100 & 2. 74400900 & -1.85179100 \\
\hline $\mathrm{C}$ & -0.25562100 & 3. 28733900 & -0.61090600 \\
\hline $\mathrm{H}$ & 1. 33287900 & 7. 13156900 & -3.01528800 \\
\hline $\mathrm{H}$ & 1. 31595000 & 4. 47354100 & 0.24346000 \\
\hline $\mathrm{C}$ & 1. 39722200 & 6. 08478400 & -3.29373400 \\
\hline $\mathrm{C}$ & 1. 54588700 & 3. 37082000 & -3.96168600 \\
\hline $\mathrm{C}$ & 2. 03151400 & 4. 32197800 & -4.84298200 \\
\hline
\end{tabular}




\begin{tabular}{lrrr}
$\mathrm{C}$ & 1.94720900 & 5.68293000 & -4.50332900 \\
$\mathrm{H}$ & 1.57040400 & 2.31319900 & -4.20165700 \\
$\mathrm{H}$ & 2.46544500 & 4.02165900 & -5.79170600 \\
$\mathrm{H}$ & 2.32150600 & 6.43567000 & -5.19225800 \\
$\mathrm{O}$ & 0.31436900 & 1.54880800 & -2.17811800 \\
$\mathrm{O}$ & 0.38874500 & 5.57424900 & -1.23355900 \\
$\mathrm{C}$ & -1.21465400 & 2.63157700 & 0.05425600 \\
$\mathrm{H}$ & -1.67446300 & 3.05411200 & 0.94220800 \\
$\mathrm{H}$ & -1.57068400 & 1.65724200 & -0.25983300 \\
$\mathrm{H}$ & -0.32077100 & 5.07544900 & 0.60175400 \\
$\mathrm{C}$ & 1.91401000 & 2.24975800 & 2.40461400 \\
$\mathrm{C}$ & 2.25228900 & 3.45753600 & 3.08076800 \\
$\mathrm{C}$ & 3.24585800 & 4.32415200 & 2.60749200 \\
$\mathrm{C}$ & 3.90846600 & 3.96255100 & 1.43765100 \\
$\mathrm{C}$ & 3.59119300 & 2.76742100 & 0.75587800 \\
$\mathrm{C}$ & 2.60268900 & 1.90836300 & 1.22725900 \\
$\mathrm{C}$ & 0.86604900 & 1.62361100 & 3.16115000 \\
$\mathrm{C}$ & 0.61724000 & 2.43909300 & 4.23327500 \\
$\mathrm{H}$ & 3.49740300 & 5.24272700 & 3.13100800 \\
$\mathrm{H}$ & 4.68687800 & 4.61145800 & 1.04466900 \\
$\mathrm{H}$ & 4.12953400 & 2.51574100 & -0.15401200 \\
$\mathrm{H}$ & 2.35888200 & 0.98643700 & 0.70738200 \\
$\mathrm{H}$ & 0.38685900 & 0.68500800 & 2.92013700 \\
$\mathrm{H}$ & -0.08795700 & 2.32513100 & 5.04617500 \\
$\mathrm{~N}$ & 1.44506300 & 3.55361300 & 4.19829600 \\
$\mathrm{C}$ & 1.48084300 & 4.62302100 & 5.17198900 \\
$\mathrm{H}$ & 2.46492500 & 4.68926800 & 5.65223200 \\
$\mathrm{H}$ & 1.25583100 & 5.59093300 & 4.70686200 \\
$\mathrm{H}$ & 0.73198300 & 4.42782600 & 5.94292500 \\
& & & \\
\hline
\end{tabular}


TS-I'-Me (Table S8, entry 4)

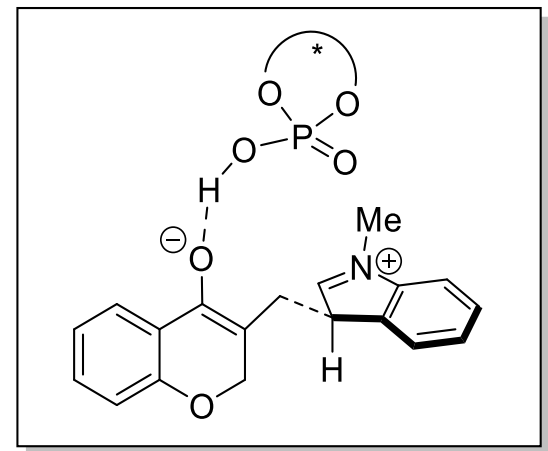

SCF energy [B3LYP-GD3BJ/6-311+G**/SMD (Chlorobenzene)]: -3330.983125670 a.u.

Thermal correction to Gibbs free energy at $258 \mathrm{~K}: 1.139596000$ a.u.

Gibbs free energy at $258 \mathrm{~K}$ [B3LYP-GD3BJ/6-311+G**/SMD (Chlorobenzene)]: -3329.843529670 a.u.

01

$\mathrm{P}$

0

0.43670000

$-1.36367300$

$-0.01980000$

0

1. 69953500

$-1.76415600$

$-1.01336700$

0

$-0.36988100$

$-2.80499900$

0. 12218000

0

$-0.41312800$

$-0.41482800$

$-0.84937200$

0

0. 87606800

$-0.97615800$

1. 35674400

C

2. 56012500

$-2.77451900$

$-0.60165500$

C

3. 72196700

$-2.46622900$

0. 13752300

4. 51856000

$-3.53378100$

0. 58494200

C

4. 17942900

$-4.86223400$

0. 33572900

3. 03988100

$-5.13685600$

$-0.41204200$

C

2. 45489000

$-6.48242400$

$-0.77847000$

C

1. 01673400

$-6.11147800$

$-1.19371400$

C

1. 13000400

$-4.66557700$

$-1.79010100$

C

2. 24233500

$-4.09675300$

$-0.91358000$

C

1. 54225900

$-4.74801300$

$-3.29644500$

0. 22275100

$-5.04608500$

$-4.04541100$

C

$-0.81452600$

$-4.38786000$

-3. 16031200

C

$-2.14942900$

-4. 10767500

$-3.43176500$

$-2.91991200$

$-3.47635600$

$-2.45631800$

C

$-2.37442300$

$-3.03682600$

$-1.23601800$

$-1.00420000$

$-3.30180500$

$-1.00826400$

$-0.25586800$

$-4.04118000$

$-1.92195100$

C

5. 42219400

$-3.30413000$

1. 14248700

4. 79955200

$-5.66747200$

0. 72219600

3. 00572100

$-6.94215100$

$-1.61190700$

H

2. 48573000

-7. 19780100

0. 05157700 


\begin{tabular}{|c|c|c|c|}
\hline $\mathrm{H}$ & 0.56776800 & -6.81956100 & -1.89760300 \\
\hline $\mathrm{H}$ & 0.37635000 & -6.07299200 & -0.30520500 \\
\hline $\mathrm{H}$ & 2. 32229200 & -5.49502500 & -3.47687200 \\
\hline $\mathrm{H}$ & 1. 93339300 & -3.77259400 & -3.6070710 \\
\hline $\mathrm{H}$ & 0.04739100 & -6.12787000 & -4.1323530 \\
\hline $\mathrm{H}$ & 0.21717200 & -4.64539700 & -5.0658920 \\
\hline $\mathrm{H}$ & -2.59641200 & -4.38744900 & -4.3826870 \\
\hline $\mathrm{H}$ & -3.97695400 & -3.31258200 & -2.6426980 \\
\hline $\mathrm{C}$ & -3.25069500 & -2.35838800 & -0.2397260 \\
\hline $\mathrm{C}$ & -4.32103200 & -1.55449000 & -0.667368 \\
\hline $\mathrm{C}$ & -3.08723200 & -2.57465200 & 1. 13856600 \\
\hline $\mathrm{C}$ & -5.23713200 & -1.00240900 & 0.23689100 \\
\hline $\mathrm{C}$ & -3.96156100 & -2.01903600 & 2. $078126 \mathrm{C}$ \\
\hline $\mathrm{C}$ & -5.03272200 & -1.24624700 & 1.6033250 \\
\hline $\mathrm{C}$ & 4. 14498200 & -1.07326200 & 0.44711400 \\
\hline $\mathrm{C}$ & 4. 21509800 & -0.09872700 & -0.5623520 \\
\hline $\mathrm{C}$ & 4. 56453900 & -0.73976100 & 1. 7373640 \\
\hline $\mathrm{C}$ & 4. 70666600 & 1. 18075800 & -0.30133500 \\
\hline $\mathrm{C}$ & 5. 06170300 & 0.53640700 & 2.0453730 \\
\hline C & 5. 12260500 & 1. 47555000 & 1. 01110700 \\
\hline $\mathrm{H}$ & -5.73997400 & -0.83866700 & 2. 32000300 \\
\hline $\mathrm{H}$ & -4.42867100 & -1.36418000 & -1.7282220 \\
\hline $\mathrm{H}$ & -2.26360400 & -3.19224500 & 1. 465082 \\
\hline $\mathrm{H}$ & 5.51290100 & 2. 46528900 & 1. 21912400 \\
\hline $\mathrm{H}$ & 4. 48498800 & -1.49381600 & 2.51439400 \\
\hline $\mathrm{H}$ & 3. 88939900 & -0.37145000 & -1.5576330 \\
\hline $\mathrm{C}$ & -6.47874300 & -0.20918600 & -0.22235700 \\
\hline $\mathrm{C}$ & -7.74430900 & -1.03297400 & 0.12091000 \\
\hline $\mathrm{H}$ & -8.64930300 & -0.50258400 & -0.2012040 \\
\hline $\mathrm{H}$ & -7.82615500 & -1.21598800 & 1. 1976100 \\
\hline $\mathrm{H}$ & -7.72246900 & -2.00624900 & -0.38191200 \\
\hline $\mathrm{C}$ & -6.55919700 & 1. 15412500 & 0.503319 \\
\hline $\mathrm{H}$ & -7.46468200 & 1. 69406000 & 0.20061600 \\
\hline $\mathrm{H}$ & -5.69772400 & 1. 78597200 & 0.25428100 \\
\hline $\mathrm{H}$ & -6.58944600 & 1. 04298600 & 1. 591957 \\
\hline $\mathrm{C}$ & -6.47939500 & 0.06104100 & -1.73929900 \\
\hline $\mathrm{H}$ & -6.51152600 & -0.86793200 & -2.31880100 \\
\hline $\mathrm{H}$ & -5.59553000 & 0.62720200 & -2.055003 \\
\hline $\mathrm{H}$ & -7.36476900 & 0.64733200 & -2.01056000 \\
\hline $\mathrm{C}$ & 4. 82217600 & 2. 25939100 & -1.396003 \\
\hline $\mathrm{C}$ & 3. 96358000 & 3. 48536400 & -1.00506500 \\
\hline $\mathrm{H}$ & 2. 90744500 & 3. 20481000 & -0.920598 \\
\hline $\mathrm{H}$ & 4. 04262400 & 4. 27232900 & -1.765494 \\
\hline $\mathrm{H}$ & 4. 28156700 & 3. 91533900 & -0.048932 \\
\hline
\end{tabular}




\begin{tabular}{|c|c|c|c|}
\hline $\mathrm{C}$ & 4. 33556000 & 1. 75189900 & -2.76699700 \\
\hline $\mathrm{H}$ & 3. 28848900 & 1. 43103500 & -2.73410000 \\
\hline $\mathrm{H}$ & 4. 93710100 & 0.90891900 & -3.12526200 \\
\hline$H_{H}$ & 4. 42057100 & 2. 55463500 & -3.50910400 \\
\hline C & 5. 53096300 & 0.84294900 & 3. 48178800 \\
\hline C & 6. 68695300 & -0.11527000 & 3.85710000 \\
\hline & 7. 03569700 & 0.08542500 & 4. 87791700 \\
\hline & 7. 53659400 & 0.01141200 & 3. 17635400 \\
\hline H & 6. 37583500 & -1.16390700 & 3. 81102800 \\
\hline & 6. 04055900 & 2. 28768000 & 3. 64237700 \\
\hline & 6. 90312800 & 2. 49064300 & 2. 99770400 \\
\hline H & 6. 35596400 & 2. 45544800 & 4. 67856300 \\
\hline $\mathrm{H}$ & 5. 26160000 & 3. 02368900 & 3. 41109400 \\
\hline$l$ & 4. 35958500 & 0.63508000 & 4. 47047500 \\
\hline $\mathrm{H}$ & 3. 96099900 & -0.38294800 & 4. 42524100 \\
\hline $\mathrm{H}$ & 3. 53436300 & 1. 32262100 & 4. 25052400 \\
\hline $\mathrm{H}$ & 4. 68995800 & 0.82207600 & 5. 49998900 \\
\hline $\mathrm{C}$ & -3.80079300 & -2.26191300 & 3.59256700 \\
\hline $\mathrm{C}$ & -5.03126400 & -3.03848800 & 4. 11920200 \\
\hline H & -5.96261300 & -2.48420200 & 3. 95910500 \\
\hline $\mathrm{H}$ & -4.93443400 & -3.22540300 & 5. 19598600 \\
\hline $\mathrm{H}$ & -5.12756900 & -4.00621100 & 3. 61399500 \\
\hline $\mathrm{C}$ & -3.70086700 & -0.90718300 & 4. 33307200 \\
\hline H & -4.58869900 & -0.28555900 & 4. 17442800 \\
\hline $\mathrm{H}$ & -2.82603700 & -0.33950400 & 3. 99548000 \\
\hline $\mathrm{H}$ & -3.60021000 & -1.06972400 & 5. 41322300 \\
\hline $\mathrm{C}$ & -2.53534400 & -3.07669900 & 3. 92462500 \\
\hline H & -2.57195000 & -4.08176300 & 3. 48991900 \\
\hline $\mathrm{H}$ & -2.44867200 & -3.19239700 & 5. 01124000 \\
\hline $\mathrm{H}$ & -1.62456700 & -2.58470800 & 3. 56560600 \\
\hline $\mathrm{C}$ & 6. 29904500 & 2. 69692700 & -1.54177400 \\
\hline $\mathrm{H}$ & 6.93268600 & 1. 84621700 & -1.81707300 \\
\hline $\mathrm{H}$ & 6. 69435200 & 3. 12004200 & -0.61217900 \\
\hline $\mathrm{H}$ & 6.39647300 & 3. 46087300 & -2.32335000 \\
\hline $\mathrm{H}$ & -0.07147100 & 0.91736700 & -1.41669000 \\
\hline $\mathrm{C}$ & -2.44895800 & 4. 30772500 & -1.18870800 \\
\hline $\mathrm{C}$ & -1.19214200 & 4. 91413100 & -3.07514900 \\
\hline $\mathrm{C}$ & -0.23786300 & 3. 91846300 & -2.77084800 \\
\hline $\mathrm{C}$ & -0.61878000 & 2. 81360700 & -1.88040300 \\
\hline $\mathrm{C}$ & -1.81664400 & 2. 93097400 & -1.17025500 \\
\hline $\mathrm{H}$ & -1.68567900 & 6. 62823200 & -4.25973400 \\
\hline $\mathrm{H}$ & -1.95323100 & 4. 99001100 & -0.48207700 \\
\hline $\mathrm{C}$ & -0.92329900 & 5. 88712300 & -4.04261800 \\
\hline C & 0.99192400 & 3. 93534400 & -3.453301( \\
\hline
\end{tabular}


1. 27222800

4. 91808900

-4. 39346300

C

0. 30722900

5. 88989600

$-4.69082400$

1. 71054800

3. 15358200

$-3.24012300$

2. 22933100

4. 92331400

$-4.90624500$

0. 51817600

6. 65628600

$-5.43195100$

0. 19793500

1. 78562200

$-1.91586600$

$-2.41310200$

4. 93208900

$-2.48354200$

$-2.45261600$

1. 87849000

$-0.46284100$

$-3.53161100$

1. 96704900

$-0.36845900$

-2. 10424600

0. 86005800

$-0.60600600$

$-3.50666000$

4. 25769400

$-0.92163300$

$-2.23810900$

3. 32696600

1. 98804800

$-0.87381000$

3. 66849200

2. 12576700

$-0.44643900$

4. 95908200

2. 42786200

$-1.43496700$

5. 92472000

2. 62633000

$-2.79817000$

5. 60058700

2. 52821200

$-3.21008300$

4. 30797300

2. 20203900

$-2.27787100$

1. 92052200

1. 58505900

$-0.94064800$

1. 46350500

1. 71400600

0. 60679600

5. 20661100

2. 51666900

$-1.14300300$

6. 94151000

2. 87216800

$-3.54235500$

6. 37150300

2. 70626900

$-4.26716100$

4. 06850900

2. 12201800

$-3.11436500$

1. 25475500

1. 76291100

$-0.52783300$

0. 46457200

1. 60465600

$-0.11879300$

2. 49392600

1. 94228800

1. 33599600

2. 38886000

2. 02933100

1. 80343900

3. 00750200

1. 25858500

1. 67073600

2. 72645400

3. 01528200

1. 61419200

1. 34383200

1. 87762200 
TS-I-Me (Table S8, entry 5)

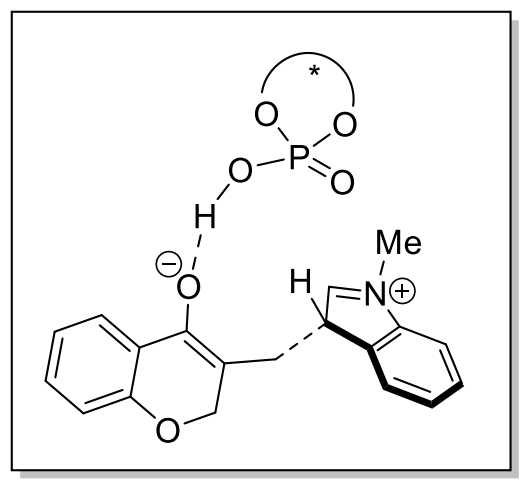

SCF energy [B3LYP-GD3BJ/6-311+G**/SMD (Chlorobenzene)]: -3330.984176060 a.u.

Thermal correction to Gibbs free energy at $258 \mathrm{~K}: 1.138146000$ a.u.

Gibbs free energy at $258 \mathrm{~K}$ [B3LYP-GD3BJ/6-311+G**/SMD (Chlorobenzene)]:

-3329.846030060 a.u.

\begin{tabular}{crrr}
01 & & & \\
$\mathrm{P}$ & 0.30795400 & 1.49081200 & 0.20846600 \\
0 & -0.62186800 & 2.46374400 & -0.76962600 \\
0 & 1.61080800 & 2.46541500 & 0.52845200 \\
0 & 0.75604900 & 0.36111700 & -0.70181100 \\
0 & -0.34969700 & 1.19476800 & 1.51375900 \\
$\mathrm{C}$ & -1.14676400 & 3.60372200 & -0.17432000 \\
$\mathrm{C}$ & -2.42657400 & 3.57076200 & 0.41698600 \\
$\mathrm{C}$ & -2.85621800 & 4.71034400 & 1.11743500 \\
$\mathrm{C}$ & -2.05092100 & 5.84100100 & 1.25099200 \\
$\mathrm{C}$ & -0.81074200 & 5.86212500 & 0.62211000 \\
$\mathrm{C}$ & 0.24041300 & 6.95099500 & 0.63386300 \\
$\mathrm{C}$ & 1.49215000 & 6.21047900 & 0.11483600 \\
$\mathrm{C}$ & 0.93253500 & 5.10339700 & -0.84100700 \\
$\mathrm{C}$ & -0.37146900 & 4.76158300 & -0.12876200 \\
$\mathrm{C}$ & 0.66148200 & 5.71512000 & -2.25638700 \\
$\mathrm{C}$ & 2.03419900 & 5.69330100 & -2.96325000 \\
$\mathrm{C}$ & 2.70430600 & 4.49110200 & -2.33448800 \\
$\mathrm{C}$ & 3.84150800 & 3.80823400 & -2.75129000 \\
$\mathrm{C}$ & 4.27197700 & 2.70475400 & -2.01810200 \\
$\mathrm{C}$ & 3.56057400 & 2.21453700 & -0.90696200 \\
$\mathrm{C}$ & 2.39493900 & 2.91670300 & -0.52196000 \\
$\mathrm{C}$ & 2.00862700 & 4.07921300 & -1.18899400 \\
$\mathrm{H}$ & -3.84485400 & 4.69720200 & 1.56775500 \\
$\mathrm{H}$ & -2.39575500 & 6.69255800 & 1.83267600 \\
$\mathrm{H}$ & -0.03748500 & 7.78110400 & -0.03163600 \\
& & & \\
\hline
\end{tabular}




\begin{tabular}{|c|c|c|c|}
\hline $\mathrm{H}$ & 0.38786300 & 7. 38382800 & 1. 63025400 \\
\hline $\mathrm{H}$ & 2. 21325100 & 6. 86674300 & -0.38343300 \\
\hline $\mathrm{H}$ & 2. 00595900 & 5. 72331700 & 0.95128300 \\
\hline $\mathrm{H}$ & 0.21452800 & 6.71317100 & -2.20243700 \\
\hline H & -0.03917800 & 5. 06302900 & -2.79016200 \\
\hline H & 2. 60070600 & 6. 61486200 & -2.76588100 \\
\hline $\mathrm{H}$ & 1. 94831100 & 5. 60531400 & -4.05268300 \\
\hline $\mathrm{H}$ & 4. 39724100 & 4. 13190600 & -3.62808200 \\
\hline 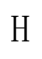 & 5. 18918300 & 2. 20259900 & -2.30977600 \\
\hline & 4. 08049700 & 1. 01932900 & -0.18565600 \\
\hline & 4. 74706600 & 0.00841200 & -0.89750400 \\
\hline C & 4. 00376100 & 0.91350100 & 1. 21239400 \\
\hline $\mathrm{C}$ & 5. 36221500 & -1.06909500 & -0.25041100 \\
\hline $\mathrm{C}$ & 4. 57584000 & -0.16406400 & 1. 89758200 \\
\hline C & 5. 25522500 & -1.13649400 & 1. 14715300 \\
\hline C & -3.34586300 & 2. 40564700 & 0.29753300 \\
\hline C & -3.63420700 & 1. 84682200 & -0.95999500 \\
\hline $\mathrm{C}$ & -4.02294900 & 1. 92379200 & 1. 42066100 \\
\hline $\mathrm{C}$ & -4.60267400 & 0.85208700 & -1.10977300 \\
\hline $\mathrm{C}$ & -4.99805900 & 0.91864500 & 1. 31803000 \\
\hline C & -5.27308800 & 0.40638900 & 0.04590000 \\
\hline $\mathrm{H}$ & 5.73203600 & -1.96088000 & 1.67026500 \\
\hline $\mathrm{H}$ & 4. 77619500 & 0.07643000 & -1.97775000 \\
\hline H & 3. 49377100 & 1. 69395100 & 1. 75820000 \\
\hline $\mathrm{H}$ & -6.03970500 & -0.35161800 & -0.06315500 \\
\hline $\mathrm{H}$ & -3.77171100 & 2. 34317400 & 2. 39002800 \\
\hline $\mathrm{H}$ & -3.09935000 & 2. 22809600 & -1.82063700 \\
\hline C & 6.17971100 & -2.13417900 & -1.01170000 \\
\hline $\mathrm{C}$ & 7. 64615000 & -2.09071500 & -0.51763200 \\
\hline $\mathrm{H}$ & 8. 25371700 & -2.83067000 & -1.05367800 \\
\hline $\mathrm{H}$ & 7. 72204900 & -2.30851500 & 0.55287600 \\
\hline $\mathrm{H}$ & 8. 08557900 & -1.10150600 & -0.68779000 \\
\hline $\mathrm{C}$ & 5. 60318000 & -3.54533600 & -0.75253700 \\
\hline $\mathrm{H}$ & 6.21540400 & -4.30717200 & -1.25090600 \\
\hline $\mathrm{H}$ & 4. 58288200 & -3.62831900 & -1.14585900 \\
\hline $\mathrm{H}$ & 5. 57804100 & -3.78715100 & 0.31543700 \\
\hline $\mathrm{C}$ & 6. 18382200 & -1.89839300 & -2.53424300 \\
\hline $\mathrm{H}$ & 6. 63286400 & -0.93378300 & -2.79538100 \\
\hline $\mathrm{H}$ & 5. 17285900 & -1.92862100 & -2.95606800 \\
\hline $\mathrm{H}$ & 6.77303200 & -2.68049600 & -3.02684000 \\
\hline $\mathrm{C}$ & -5.00344700 & 0.29061800 & -2.48917300 \\
\hline $\mathrm{C}$ & -4.97410900 & -1.25500400 & -2.47852000 \\
\hline $\mathrm{H}$ & -3.97152700 & -1.63244300 & -2.25234700 \\
\hline $\mathrm{H}$ & -5.26563700 & -1.64493000 & -3.46139700 \\
\hline
\end{tabular}


$-5.66669200$

$-4.06326400$

$-3.02248600$

-4. 10220200

$-4.36237400$

$-5.73010100$

$-6.44676600$

$-6.97754400$

$-7.17880900$

$-5.74109100$

$-6.78915300$

$-7.57056000$

$-7.27525800$

$-6.34731000$

$-4.70510200$

$-3.92601200$

$-4.21138300$

$-5.20364300$

4. 51363600

5. 94727000

6. 58331400

5. 92467500

6. 42274100

3. 87886400

4. 44507600

2. 85046700

3. 84705000

3. 67285800

4. 10990600

3. 62735100

2. 64555600

$-6.43926000$

$-6.49242500$

$-7.16133000$

$-6.75522200$

0. 28711600

$-2.59088600$

$-1.92993000$

$-0.76955200$

$-0.72882300$

$-1.69747600$

$-2.97618100$

$-2.11048700$

$-2.06537600$
$-1.67752500$

0. 77194300

0. 48935600

1. 85898900

0. 31742700

0. 43816400

1. 63683500

1. 31165700

2. 08216900

2. 42115900

$-0.64052200$

$-0.26926900$

$-0.94853700$

$-1.53555400$

-0. 15577300

0. 56625600

$-1.03550100$

$-0.46912600$

$-0.28477700$

$-0.21033100$

$-1.01574800$

$-0.29296900$

0. 74228700

$-1.63890800$

$-2.49001400$

$-1.71262400$

$-1.74068700$

0. 83561400

1. 82498000

0. 68403900

0. 84780800

0. 76599300

1. 86035600

0. 41641100

0.38348800

$-0.92678300$

$-3.84206300$

$-4.58953000$

$-3.78693600$

$-2.67289900$

$-2.62418600$

-6. 16388200

$-4.64605200$

$-5.57453700$
$-1.74277200$

$-3.61140900$

$-3.41794700$

$-3.74151600$

$-4.56296200$

2. 58723600

3. 25427900

4. 15783700

2. 57101100

3. 54572300

2. 28912600

1. 61655000

3. 22210900

1. 83584200

3. 58210200

3. 84579400

3. 15003600

4. 50808500

3. 43418500

4. 01175200

3. 62836800

5. 10558700

3. 75249500

3. 83024300

3. 43600100

3. 45764100

4. 92196500

4. 07722500

3. 90278300

5. 16193700

3. 69656600

$-2.82005000$

$-2.84864900$

$-2.07382700$

$-3.79889600$

$-1.29789300$

$-0.49807700$

$-2.62962800$

$-2.57620300$

$-1.62509500$

$-0.62115500$

$-3.63623300$

0. 08007400

$-3.61151900$ 
$0.25490400-4.00918300 \quad-3.51352500$

0. 13362100

$-5.00749900$

$-4.47174500$

C

$-1.03332000$

$-5.78216900$

$-4.52203200$

$\mathrm{H}$

1. 13152000

$-3.37150300$

$-3.47932000$

0.93289600

$-5.17677000$

$-5.18684800$

$-1.13689900$

$-6.55772200$

$-5.27622500$

0. 23236100

$-1.80589200$

$-1.84406000$

$-2.96590000$

$-4.39412400$

$-1.77274400$

$-1.80477300$

$-1.56654600$

0. 30970000

$-2.73620500$

$-1.48745100$

0. 86328800

$-1.35508700$

$-0.60751900$

0. 09603700

$-3.53161800$

$-3.58701700$

$-0.00623200$

$-1.01944000$

$-3.18398800$

2. 50472500

$-0.00325100$

$-4.02369000$

1. 99397100

$-0.01181300$

$-5.40751600$

2. 15817500

$-1.07314900$

$-5.95313500$

2. 88154900

$-2.08247200$

$-5.13638900$

3. 41886500

$-2.06899600$

$-3.75444000$

3. 23173600

$-0.70875200$

$-1.82969600$

2. 05565500

0. 56924200

$-1.92011200$

1. 45881000

0. 77145600

$-6.03867000$

1. 74969700

$-1.11388300$

$-7.02717700$

3. 03738300

$-2.88738400$

$-5.59191200$

3. 98845600

$-2.85574500$

$-3.13210500$

3. 64933200

$-1.05208000$

$-0.89855100$

2. 48879000

1. 15198400

$-1.12558900$

1. 01204900

0. 94219400

$-3.20532900$

1. 35064700

2. 13949100

$-3.68387900$

0. 67316300

2. 72791900

$-4.30487500$

1. 35480900

1. 86659800

$-4.27105100$

$-0.20931900$

2. 73736700

$-2.82555200$

0. 36700700 
TS-III-S (4ab) (Table S8, entry 6)

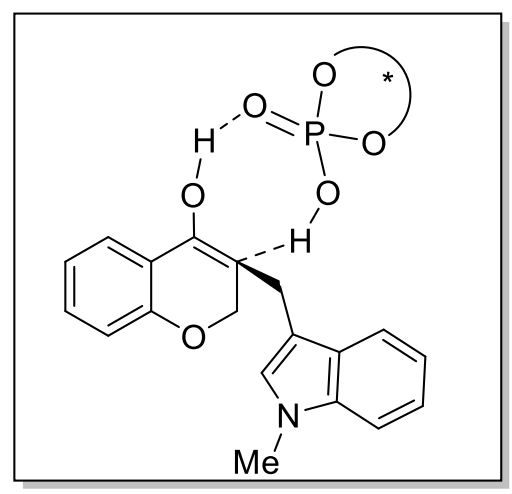

SCF energy [B3LYP-GD3BJ/6-311+G**/SMD (Chlorobenzene)]: -3330.992557610 a.u.

Thermal correction to Gibbs free energy at $258 \mathrm{~K}: 1.136364000$ a.u.

Gibbs free energy at $258 \mathrm{~K}$ [B3LYP-GD3BJ/6-311+G**/SMD (Chlorobenzene)]: -3329.856193610 a.u.

01

$\mathrm{P}$

0

0.44786600

1. 42897400

$-0.10123200$

1. 76627700

2. 23175800

0. 44512500

0

$-0.52546700$

2. 60888100

$-0.68398100$

0

$-0.16544000$

0. 82623200

1. 18434500

0

0. 73817200

0. 48345100

$-1.23016500$

2. 53692500

2. 89256100

$-0.52195500$

C

3. 63911600

2. 24292600

$-1.11120600$

C

4. 28926900

2. 90102600

-2. 16933600

C

3. 86605400

4. 14364900

$-2.63903400$

C

2. 80746200

4. 78100800

$-2.00137800$

2. 16718500

6. 11420900

$-2.32121500$

C

0. 84501800

6. 04963200

$-1.52508100$

1. 16847700

5. 14347100

$-0.28888300$

2. 16451200

4. 17569300

$-0.91158100$

1. 84159000

6. 00051800

0. 83575200

0. 66368700

6. 65629800

1. 58739200

$-0.44086700$

5. 63676100

1. 41691900

$-1.64840600$

5. 53937000

2. 09853200

$-2.52826800$

4. 51062500

1. 76889900

$-2.20656900$

3. 52513500

0. 81821500

$-0.94881200$

3. 62018400

0. 18708500

$-0.10450600$

4. 70324000

0. 42457000

$-0.39182000$

$-0.35643800$

1. 05868100

5. 14507500

2. 41476500

$-2.62841700$

4. 36804000

4. 60756600

$-3.48452800$

2. 79708200

6. 94959600

$-1.98338500$ 


\begin{tabular}{|c|c|c|c|}
\hline $\mathrm{H}$ & 2. 00613000 & 6.25731300 & -3.39591900 \\
\hline $\mathrm{H}$ & 0.46334300 & 7. 03346700 & -1.23374800 \\
\hline $\mathrm{H}$ & 0.07392100 & 5. 55770600 & -2.12869300 \\
\hline H & 2. 56037200 & 6. 72259300 & 0.43549200 \\
\hline 4 & 2. 38357500 & 5. 32935900 & 1. 51158200 \\
\hline $\mathrm{H}$ & 0.38787500 & 7. 62029900 & 1. 13668900 \\
\hline $\mathrm{H}$ & 0.89275200 & 6. 85656000 & 2. 64045200 \\
\hline & -1.91596600 & 6. 26265400 & 2. 86487100 \\
\hline H & -3.49975400 & 4. 46200700 & 2. 25126800 \\
\hline & -3.23022900 & 2. 49748000 & 0.48197000 \\
\hline & -4.00577800 & 1. 92185700 & 1. 49939100 \\
\hline$C$ & -3.53369500 & 2. 19858900 & -0.85476100 \\
\hline C & -5.09583500 & 1. 09335100 & 1. 20920500 \\
\hline $\mathrm{C}$ & -4.61278700 & 1. 37280800 & -1.19012700 \\
\hline C & -5.38011100 & 0.84174100 & -0.14143800 \\
\hline C & 4. 16484000 & 0.93445200 & -0.63695900 \\
\hline C & 4. 42877400 & 0.72182300 & 0.71843800 \\
\hline $\mathrm{C}$ & 4. 50366000 & -0.06586000 & -1.56297800 \\
\hline $\mathrm{C}$ & 5. 04761100 & -0.45421500 & 1. 16887600 \\
\hline $\mathrm{C}$ & 5. 11196600 & -1.25490200 & -1.15813600 \\
\hline $\mathrm{C}$ & 5. 38112100 & -1.42089900 & 0.21364900 \\
\hline $\mathrm{C}$ & -1.36290100 & -2.16878300 & 2.14513400 \\
\hline $\mathrm{C}$ & 0.16121600 & -3.94436300 & 2. 36585900 \\
\hline C & 1. 07867500 & -3.26221300 & 1.52927100 \\
\hline $\mathrm{C}$ & 0.54647800 & -2.26088300 & 0.62343200 \\
\hline $\mathrm{C}$ & -0.73649400 & -1.71770600 & 0.83378900 \\
\hline $\mathrm{H}$ & -0.15360200 & -5.59281800 & 3. 69951500 \\
\hline H & -0.97833400 & -1.57817300 & 2. 98855600 \\
\hline $\mathrm{C}$ & 0.57269000 & -5.07534600 & 3. 08152800 \\
\hline $\mathrm{C}$ & 2. 41502300 & -3.70493700 & 1. 46940000 \\
\hline C & 2. 82617100 & -4.80488600 & 2. 20653300 \\
\hline $\mathrm{C}$ & 1. 89564000 & -5.49287500 & 3. 00228700 \\
\hline $\mathrm{H}$ & 3. 10533500 & -3.17044400 & 0.82655700 \\
\hline $\mathrm{H}$ & 3. 85737200 & -5.14141900 & 2. 15930900 \\
\hline $\mathrm{H}$ & 2. 21269100 & -6.36124000 & 3. 57394000 \\
\hline 0 & 1. 32094200 & -1.94037700 & -0.39856900 \\
\hline 0 & -1.12764300 & -3.55940000 & 2. 47185100 \\
\hline $\mathrm{C}$ & -1.69410000 & -1.54592100 & -0.37742200 \\
\hline $\mathrm{H}$ & -1.12193300 & -1.18071000 & -1.23484600 \\
\hline $\mathrm{H}$ & -2.40983000 & -0.75168400 & -0.13857000 \\
\hline $\mathrm{C}$ & -2.44221900 & -2.80153800 & -0.73130900 \\
\hline $\mathrm{C}$ & -1.94596100 & -3.97208500 & -1.41768700 \\
\hline $\mathrm{C}$ & -3.75728200 & -3.06783800 & -0.43418300 \\
\hline C & -3.02359100 & -4.89897800 & -1.49412700 \\
\hline
\end{tabular}


$-4.12138900$

$-0.71071000$

$-0.58016500$

0. 37175000

$-1.66042400$

$-1.52771900$

$-2.89647500$

0. 13207000

$-3.73015500$

$-2.44871500$

$-4.48472300$

1. 05109700

-6. 23803000

$-3.74291500$

$-2.92806600$

5. 86972800

4. 26796100

4. 16896700

$-6.00573100$

$-7.41732300$

$-8.08708800$

$-7.86403100$

$-7.37656100$

-6. 11488700

$-6.76960500$

$-5.13259300$

$-6.53386600$

$-5.48082900$

$-5.44498100$

$-4.47684200$

-6. 14650800

5. 36306400

6. 01338900

5. 35632600

6. 21670000

6. 96717100

4. 05788500

3. 35324600

3. 55522700

4. 27516800

5. 49859000

7. 02791300

7. 31841600

7. 36390200
$-4.32476100$

-4. 32576200

$-5.56520100$

$-5.84497000$

$-6.46728100$

$-7.42878600$

$-6.14680300$

$-3.64190800$

$-6.84161700$

$-2.06800300$

$-2.43862900$

$-1.05912600$

0. 22300300

2. 13931700

2. 64648200

$-2.33329200$

0. 10459900

1. 50276500

0. 51129900

1. 13210100

0. 73439000

0. 91468600

2. 22133700

$-1.02413800$

$-1.43625500$

$-1.50041300$

$-1.31411500$

0. 81287500

1. 88884300

0. 40305000

0. 36086900

$-0.61390000$

$-1.97109400$

$-2.80952200$

$-2.03082100$

$-2.10618900$

$-0.49702700$

$-1.29164400$

0. 46182000

$-0.58464600$

$-2.36647900$

$-2.59586300$

$-3.39130800$

$-2.89082100$
$-0.88132800$

$-1.98781800$

$-2.60419500$

$-3.04746800$

$-2.66549600$

$-3.15434200$

$-2.11394300$

$-1.94875300$

-2. 16723900

2. 11488700

0. 06077400

$-0.82322000$

$-0.38769100$

2. 52771700

$-1.63145700$

0. 53697800

$-2.60662500$

1. 42529500

2. 31060000

2. 17665600

2. 94938000

1. 20067700

2. 28927500

2. 16476900

2. 94234200

2. 26681900

1. 19494900

3. 72787100

3. 93085200

3. 88706300

4. 47198500

2. 66941100

2. 99733700

2. 74003000

4. 07263500

2. 47436000

3. 49194700

3. 22285900

3. 33057900

4. 56389000

$-2.15362900$

$-2.10300000$

$-2.80058000$

$-1.10330400$ 


\begin{tabular}{lrrr}
$\mathrm{H}$ & 7.56999800 & -1.68493800 & -2.38200900 \\
$\mathrm{C}$ & 4.77099100 & -3.67827800 & -1.77566900 \\
$\mathrm{H}$ & 5.05927900 & -4.03116300 & -0.77944700 \\
$\mathrm{H}$ & 5.01866600 & -4.47268900 & -2.49075000 \\
$\mathrm{H}$ & 3.68444200 & -3.53776500 & -1.78124800 \\
$\mathrm{C}$ & 5.11637800 & -2.01379500 & -3.60385700 \\
$\mathrm{H}$ & 5.62748800 & -1.10910200 & -3.95228000 \\
$\mathrm{H}$ & 4.03714800 & -1.86146700 & -3.71533000 \\
$\mathrm{H}$ & 5.40640900 & -2.83348400 & -4.27104800 \\
$\mathrm{C}$ & -5.00936800 & 1.09149300 & -2.65388400 \\
$\mathrm{C}$ & -5.11566200 & -0.43162000 & -2.89760100 \\
$\mathrm{H}$ & -4.16287200 & -0.93442200 & -2.70166500 \\
$\mathrm{H}$ & -5.39712000 & -0.62742500 & -3.93956600 \\
$\mathrm{H}$ & -5.87479900 & -0.89783200 & -2.26029000 \\
$\mathrm{C}$ & -3.98690200 & 1.66058900 & -3.65680600 \\
$\mathrm{H}$ & -2.98461300 & 1.24895100 & -3.49463200 \\
$\mathrm{H}$ & -3.92086500 & 2.75289400 & -3.60021800 \\
$\mathrm{H}$ & -4.29210000 & 1.40422600 & -4.67766500 \\
$\mathrm{C}$ & -6.38246400 & 1.74788500 & -2.93609000 \\
$\mathrm{H}$ & -7.16322700 & 1.35341600 & -2.27646000 \\
$\mathrm{H}$ & -6.69157200 & 1.55906600 & -3.97173100 \\
$\mathrm{H}$ & -6.33638600 & 2.83283600 & -2.78731000 \\
$\mathrm{C}$ & 6.34269700 & 0.50296100 & 3.10379400 \\
$\mathrm{H}$ & 5.92194700 & 1.49981500 & 2.93663900 \\
$\mathrm{H}$ & 7.28321900 & 0.43904800 & 2.54432500 \\
$\mathrm{H}$ & 6.57522300 & 0.41257700 & 4.17225100 \\
$\mathrm{C}$ & -5.43166100 & -4.92726000 & -0.76444200 \\
$\mathrm{H}$ & -5.87658500 & -5.10792400 & -1.75095600 \\
$\mathrm{H}$ & -5.37818000 & -5.88128800 & -0.22633700 \\
$\mathrm{H}$ & -6.08448700 & -4.25232600 & -0.20654100 \\
& & & \\
\hline
\end{tabular}


TS-III-R (4ab) (Table S8, entry 7)

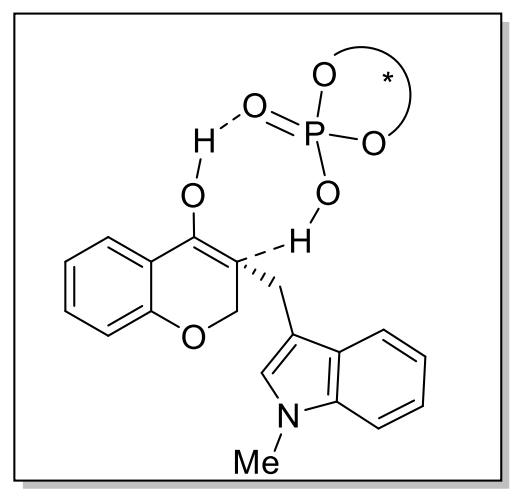

SCF energy [B3LYP-GD3BJ/6-311+G**/SMD (Chlorobenzene)]: -3330.987769880 a.u.

Thermal correction to Gibbs free energy at $258 \mathrm{~K}: 1.136585000$ a.u.

Gibbs free energy at $258 \mathrm{~K}$ [B3LYP-GD3BJ/6-311+G**/SMD (Chlorobenzene)]: -3329.851184880 a.u.

$\begin{array}{rrrr}01 & & & \\ \mathrm{P} & 0.61043200 & 1.37576200 & 0.04547700 \\ 0 & -0.22406000 & 2.58391500 & -0.68527300 \\ \mathrm{O} & 2.04332200 & 2.06843600 & 0.42363100 \\ \mathrm{O} & 0.83185100 & 0.32296500 & -1.06177700 \\ \mathrm{O} & -0.04327100 & 0.91571400 & 1.31600500 \\ \mathrm{C} & -0.48730300 & 3.72050600 & 0.09386200 \\ \mathrm{C} & -1.68029400 & 3.81031200 & 0.83571100 \\ \mathrm{C} & -1.84378400 & 4.91646000 & 1.68596700 \\ \mathrm{C} & -0.86343100 & 5.89816900 & 1.81591800 \\ \mathrm{C} & 0.29040300 & 5.80039500 & 1.04720500 \\ \mathrm{C} & 1.49710900 & 6.71235100 & 1.03677800 \\ \mathrm{C} & 2.56085500 & 5.84213200 & 0.33590800 \\ \mathrm{C} & 1.74751000 & 4.93310200 & -0.64811600 \\ \mathrm{C} & 0.47061300 & 4.73111400 & 0.15619500 \\ \mathrm{C} & 1.47956600 & 5.70633100 & -1.98150700 \\ \mathrm{C} & 2.77908400 & 5.53444800 & -2.79803200 \\ \mathrm{C} & 3.29190200 & 4.19335500 & -2.31920900 \\ \mathrm{C} & 4.28173000 & 3.38914000 & -2.87161400 \\ \mathrm{C} & 4.58934100 & 2.17841100 & -2.25511100 \\ \mathrm{C} & 3.88242400 & 1.70233800 & -1.13516000 \\ \mathrm{C} & 2.85480500 & 2.52429100 & -0.62562900 \\ \mathrm{C} & 2.61146600 & 3.78749800 & -1.16132800 \\ \mathrm{H} & 0.37538100 & -0.79092700 & -0.75939500 \\ \mathrm{H} & -2.76277700 & 4.98861100 & 2.26057100 \\ \mathrm{H} & -1.00280200 & 6.72391900 & 2.50929700 \\ \mathrm{H} & 1.29579900 & 7.63251500 & 0.46963300\end{array}$




\begin{tabular}{|c|c|c|c|}
\hline $\mathrm{H}$ & 1. 79970900 & 7. 02291900 & 2. 04338300 \\
\hline $\mathrm{H}$ & 3. 33391900 & 6. 42460900 & -0.17520400 \\
\hline $\mathrm{H}$ & 3. 05781300 & 5. 20206600 & 1. 07362500 \\
\hline 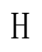 & 1. 20739700 & 6. 75263100 & -1.80931100 \\
\hline H & 0.64573800 & 5. 22580200 & -2.50579200 \\
\hline H & 3. 49761300 & 6. 33750600 & -2.58087300 \\
\hline $\mathrm{H}$ & 2. 60416400 & 5. 55492300 & -3.88002300 \\
\hline & 4. 82250000 & 3. 70156000 & -3.76166600 \\
\hline H & 5. 40065800 & 1. 57289600 & -2.64663400 \\
\hline$\sim$ & 4. 27602100 & 0.39831400 & -0.53622800 \\
\hline c & 4. 68413400 & -0.65363000 & -1.37010500 \\
\hline C & 4. 35059900 & 0.22824400 & 0.85424300 \\
\hline C & 5. 18774400 & -1.84944500 & -0.84961800 \\
\hline $\mathrm{C}$ & 4. 82924000 & -0.95970700 & 1. 41663000 \\
\hline $\mathrm{C}$ & 5. 24585500 & -1.97662600 & 0.54529500 \\
\hline C & -2.78063600 & 2. 80869200 & 0.76130800 \\
\hline C & -3.46175700 & 2. 58027600 & -0.43563500 \\
\hline $\mathrm{C}$ & -3.21926600 & 2. 17529700 & 1. 93380700 \\
\hline $\mathrm{C}$ & -4.58143200 & 1. 73456900 & -0.48739300 \\
\hline $\mathrm{C}$ & -4.31863200 & 1. 31504800 & 1. 92559300 \\
\hline $\mathrm{C}$ & -4.97801500 & 1. 10739000 & 0.69998400 \\
\hline $\mathrm{C}$ & 0.04919600 & -3.01087700 & -1.57776800 \\
\hline $\mathrm{C}$ & 1. 13278900 & -4.20026600 & 0.15361500 \\
\hline C & 0.41416700 & -3.49520000 & 1. 14295400 \\
\hline $\mathrm{C}$ & -0.42017000 & -1.93058900 & -0.60703400 \\
\hline $\mathrm{H}$ & 2. 39747200 & -5.87495000 & -0.28329400 \\
\hline $\mathrm{H}$ & -0.75121400 & -3.73622000 & -1.77721700 \\
\hline $\mathrm{C}$ & 1. 85019200 & -5.35175300 & 0.49301500 \\
\hline $\mathrm{C}$ & 0.43753600 & -3.95675200 & 2. 47268700 \\
\hline $\mathrm{C}$ & 1. 13049300 & -5.11209800 & 2. 80355400 \\
\hline $\mathrm{C}$ & 1. 83914700 & -5.80280400 & 1.80904700 \\
\hline $\mathrm{H}$ & -0.10091700 & -3.39214000 & 3.22589700 \\
\hline $\mathrm{H}$ & 1. 13149200 & -5.47450100 & 3. 82682400 \\
\hline $\mathrm{H}$ & 2. 38695400 & -6.70583600 & 2. 06514400 \\
\hline 0 & -0.69961600 & -1.52264100 & 1. 75057400 \\
\hline 0 & 1. 20212300 & -3.74679800 & -1.12035500 \\
\hline $\mathrm{C}$ & -1.73456700 & -1.23092300 & -1.06467500 \\
\hline $\mathrm{H}$ & -1.49077700 & -0.51166000 & -1.85300200 \\
\hline $\mathrm{H}$ & -2.12571800 & -0.64039600 & -0.22946000 \\
\hline $\mathrm{C}$ & -2.78325500 & -2.17944200 & -1.58031200 \\
\hline $\mathrm{C}$ & -3.56269200 & -3.14598900 & -0.84346500 \\
\hline $\mathrm{C}$ & -3.16268000 & -2.31495500 & -2.89547100 \\
\hline $\mathrm{C}$ & -4.39478500 & -3.81738900 & -1.78348200 \\
\hline $\mathrm{N}$ & -4.12894600 & -3.29332500 & -3.03504200 \\
\hline
\end{tabular}




\begin{tabular}{|c|c|c|c|}
\hline C & -3.64041600 & -3.51381600 & 0.51105800 \\
\hline C & -4.52888500 & -4.51360700 & 0.89038500 \\
\hline $\mathrm{H}$ & -4.59598300 & -4.80228600 & 1. 93568500 \\
\hline C & -5.34751000 & -5.15969100 & -0.05728300 \\
\hline H & -6.03401100 & -5.93579400 & 0.27001700 \\
\hline C & -5.29384000 & -4.82071800 & -1.40501700 \\
\hline 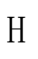 & -3.02258100 & -3.01805700 & 1. 25327000 \\
\hline H & -5.92792100 & -5.31912200 & -2.13294300 \\
\hline H & 0.35563300 & -2.57006300 & -2.52971500 \\
\hline 1 & -2.80989300 & -1.77774200 & -3.76663200 \\
\hline H & -0.64367800 & -0.52662700 & 1. 52982800 \\
\hline $\mathrm{H}$ & -3.12679100 & 3. 10083700 & -1.32707700 \\
\hline H & -5.83275700 & 0.44157600 & 0.68157300 \\
\hline $\mathrm{H}$ & -2.66956800 & 2. 36460600 & 2.84771200 \\
\hline $\mathrm{H}$ & 4. 04086900 & 1. 04533700 & 1. 49121300 \\
\hline $\mathrm{H}$ & 5. 62959000 & -2.89985900 & 0.96945500 \\
\hline H & 4. 59416900 & -0.52321300 & -2.44157500 \\
\hline $\mathrm{C}$ & -5.38349800 & 1. 60070200 & -1.79770300 \\
\hline C & -6.16013100 & 2. 92012700 & -2.03018000 \\
\hline H & -6.84856400 & 3. 12122400 & -1.20138900 \\
\hline $\mathrm{H}$ & -6.74755200 & 2. 86379700 & -2.95563800 \\
\hline $\mathrm{H}$ & -5.47923300 & 3. 77433900 & -2.11337700 \\
\hline $\mathrm{C}$ & -6.39716200 & 0.44021500 & -1.74873700 \\
\hline H & -7.17424600 & 0.60306100 & -0.99372700 \\
\hline $\mathrm{H}$ & -5.90652300 & -0.51608500 & -1.53521100 \\
\hline $\mathrm{H}$ & -6.90261000 & 0.35175600 & -2.71761300 \\
\hline $\mathrm{C}$ & -4.44264300 & 1. 35476800 & -2.99834500 \\
\hline H & -3.87541800 & 0.42834700 & -2.86343500 \\
\hline H & -3.72894300 & 2. 17144900 & -3.14451600 \\
\hline $\mathrm{H}$ & -5.02911000 & 1. 26475900 & -3.92133100 \\
\hline C & 4. 93005700 & -1.16914800 & 2.94063600 \\
\hline $\mathrm{C}$ & 5.68535000 & -2.99854100 & -1.74871300 \\
\hline $\mathrm{C}$ & 6. 40792300 & -1.40765500 & 3. 33182300 \\
\hline $\mathrm{H}$ & 6. 82478900 & -2.29021600 & 2.83468300 \\
\hline $\mathrm{H}$ & 6. 49701600 & -1.56127500 & 4. 41458700 \\
\hline H & 7. 02907400 & -0.54668000 & 3. 05902200 \\
\hline 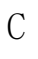 & 4. 08750500 & -2.40026100 & 3. 34867600 \\
\hline H & 4. 42464000 & -3.31285300 & 2.84605000 \\
\hline $\mathrm{H}$ & 3. 03194500 & -2.25360400 & 3. 09606100 \\
\hline & 4. 15871500 & -2.56917700 & 4. 4305800 \\
\hline $\mathrm{C}$ & 4. 41241200 & 0.04523000 & 3. 73558600 \\
\hline H & 3. 36226200 & 0.26211000 & 3. 510986 \\
\hline & 4. 99870200 & 0.94822900 & $3.5304030 c$ \\
\hline & 4. 48829400 & -0.15901100 & 4. 809929 \\
\hline
\end{tabular}




$\begin{array}{lrrr}\mathrm{C} & 7.17643900 & -3.27803800 & -1.44473000 \\ \mathrm{H} & 7.54837700 & -4.09523400 & -2.07549300 \\ \mathrm{H} & 7.33310500 & -3.56638700 & -0.40003200 \\ \mathrm{H} & 7.78982800 & -2.39095100 & -1.64070700 \\ \mathrm{C} & 5.55996100 & -2.66922400 & -3.24874500 \\ \mathrm{H} & 6.14790300 & -1.78630200 & -3.52467900 \\ \mathrm{H} & 4.51901900 & -2.49269100 & -3.54181300 \\ \mathrm{H} & 5.93212500 & -3.51206700 & -3.84254600 \\ \mathrm{C} & 4.85637700 & -4.27344200 & -1.47119300 \\ \mathrm{H} & 4.93820400 & -4.59046500 & -0.42602000 \\ \mathrm{H} & 5.20740200 & -5.10175900 & -2.10023500 \\ \mathrm{H} & 3.79678500 & -4.10009300 & -1.68632800 \\ \mathrm{C} & -0.27538400 & -2.27601900 & 0.75326100 \\ \mathrm{C} & -4.81824700 & 0.60534700 & 3.19974200 \\ \mathrm{C} & -4.62962900 & -0.92105500 & 3.04294200 \\ \mathrm{H} & -5.16642800 & -1.31302000 & 2.17319500 \\ \mathrm{H} & -5.00057600 & -1.44595700 & 3.93268600 \\ \mathrm{H} & -3.56880500 & -1.16778100 & 2.91989800 \\ \mathrm{C} & -4.05068200 & 1.05379400 & 4.45862100 \\ \mathrm{H} & -4.14650900 & 2.13176600 & 4.63230300 \\ \mathrm{H} & -2.98429400 & 0.81097100 & 4.39569700 \\ \mathrm{H} & -4.45401600 & 0.53871400 & 5.33809200 \\ \mathrm{C} & -6.31717200 & 0.91771500 & 3.42180400 \\ \mathrm{H} & -6.67722200 & 0.42175700 & 4.33162500 \\ \mathrm{H} & -6.93829500 & 0.57088400 & 2.58978000 \\ \mathrm{H} & -6.48091200 & 1.99564800 & 3.53538600 \\ \mathrm{C} & -4.79565000 & -3.66750100 & -4.26480100 \\ \mathrm{H} & -4.71110200 & -4.74633000 & -4.43901400 \\ \mathrm{H} & -5.85899000 & -3.39885400 & -4.24021300 \\ \mathrm{H} & -4.32337100 & -3.14432300 & -5.09909500\end{array}$


TS-III-S (4ad) (Table S8, entry 8)

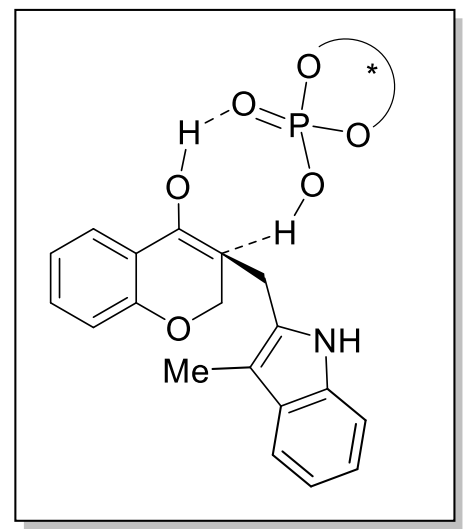

SCF energy [B3LYP-GD3BJ/6-311+G**/SMD (Chlorobenzene)]: -3331.006012900 a.u.

Thermal correction to Gibbs free energy at $258 \mathrm{~K}: 1.270908000$ a.u. Gibbs free energy at $258 \mathrm{~K}$ [B3LYP-GD3BJ/6-311+G**/SMD (Chlorobenzene)]: -3329.868628900 a.u.

01

$\mathrm{P}$

0

0. 86447300

1. 26833500

$-0.06730600$

2. 29285100

1. 83614300

0. 48942400

0

0. 08393700

2. 59990200

$-0.61209500$

0

0. 16784300

0.73431500

1. 20570600

0

0.99664500

0.32197800

$-1.22513100$

C

3. 15348000

2. 39656900

$-0.46583200$

C

4. 12870600

1. 59662100

$-1.09208700$

C

4. 87393100

2. 17963100

$-2.13133100$

C

4. 65748800

3. 49189000

$-2.55034700$

C

3. 71666500

4. 26787800

$-1.88190000$

C

3. 29266600

5. 69502400

$-2.15319000$

1. 97674000

5. 80976500

$-1.35248000$

C

2. 15623000

4. 82521200

$-0.14755300$

2. 98904800

3. 73521300

$-0.80758300$

2. 95442700

5. 52893800

1. 00058100

1. 89370600

6. 33757200

1. 77746900

0. 64287200

5. 50972100

1. 58024700

$-0.56558400$

5. 58246900

2. 26324700

$-1.59654100$

4. 71623800

1. 90581300

$-1.43345700$

3. 72191500

0. 92424300

$-0.17573000$

3. 63814900

0. 29072800

0. 82888000

4. 56641300

0. 55795800

$-0.26007200$

$-0.38870600$

1. 03819400

5. 63535700

1. 57705900

$-2.61774900$

H

5. 22502900

3. 89950000

$-3.38325000$ 


\begin{tabular}{|c|c|c|c|}
\hline $\mathrm{H}$ & 4. 04507000 & 6. 40998300 & -1.79103900 \\
\hline H & 3. 15568900 & 5. 89776500 & -3.22152900 \\
\hline & 1. 75125000 & 6. 83060700 & -1.02779000 \\
\hline & 1. 13912000 & 5. 46241200 & -1.96788800 \\
\hline & 3. 77853700 & 6. 14107000 & 0.62072100 \\
\hline & 3. 38274700 & 4. 75922100 & 1. 65248700 \\
\hline & 1. 77197900 & 7. 34610000 & 1.35743500 \\
\hline & 2. 15059400 & 6. 46670900 & 2. 83513400 \\
\hline & -0.71682300 & 6. 31487600 & 3. 05230800 \\
\hline & -2.56389100 & 4. 80871600 & 2. 38999600 \\
\hline & -2.60859500 & 2. 88148700 & 0.56047200 \\
\hline & -3.47414100 & 2. 41622000 & 1. 56164400 \\
\hline & -2.95321500 & 2. 67400500 & -0.78443300 \\
\hline & -4.68979000 & 1. 79433500 & 1. 25015700 \\
\hline & -4.15479100 & 2. 05245900 & -1.14318800 \\
\hline & -5.00524900 & 1. 62653700 & -0.10814000 \\
\hline & 4. 41895800 & 0.19630600 & -0.68085400 \\
\hline & 4. 64062600 & -0.12232700 & 0.66121400 \\
\hline & 4. 56811100 & -0.80583600 & -1.65380900 \\
\hline & 5. 02901500 & -1.41230400 & 1. 05373300 \\
\hline & 4. 94625600 & -2.10373300 & -1.30755900 \\
\hline & 5. 17756500 & -2.37942800 & 0.05356100 \\
\hline & -1.56143200 & -2.03155600 & 2. 03516100 \\
\hline & -0.42284200 & -4.08236100 & 2. 13879900 \\
\hline & 0.61692900 & -3.54556400 & 1.33959300 \\
\hline & 0.29632700 & -2.41272300 & 0.49255900 \\
\hline & -0.85630100 & -1.63778400 & 0.74424900 \\
\hline & -1.06487600 & -5.71380400 & 3. 37279700 \\
\hline & -1.05991800 & -1.58508100 & 2. 90524100 \\
\hline & -0.24624400 & -5.31241200 & 2. 78477200 \\
\hline$C$ & 1. 84393500 & -4.23433000 & 1. 25207800 \\
\hline & 2. 02677000 & -5.43274500 & 1. 92385800 \\
\hline & 0.97176900 & -5.97237500 & 2. 67882800 \\
\hline & 2. 63109600 & -3.80694000 & 0.64084700 \\
\hline & 2. 97400600 & -5.95908100 & 1.85738700 \\
\hline & 1. 10928800 & -6.91703500 & 3. 19838900 \\
\hline 0 & 1. 11889100 & -2.19761700 & -0.51638300 \\
\hline 0 & -1.61130900 & -3.45817700 & 2.27188000 \\
\hline & -1.74159200 & -1.20506300 & -0.45532800 \\
\hline & -1.10293500 & -1.02142500 & -1.32180300 \\
\hline & -2.20068100 & -0.23696600 & -0.22305100 \\
\hline & -2.77835600 & -3.34874300 & -1.55608400 \\
\hline & -4.11470700 & -3.88925100 & -1.55505600 \\
\hline & -2.81857400 & -2.19022100 & -0.81003400 \\
\hline
\end{tabular}


$-4.92937000$

$-4.10970500$

$-3.01167200$

$-2.00528700$

$-4.70121400$

$-5.02704700$

$-6.06120000$

$-5.25208400$

$-6.52494600$

-6. 12772800

$-6.85276200$

$-4.36127200$

$-7.91406100$

-6. 29848900

$-4.56113600$

$-3.23218000$

$-4.09965100$

$-5.72045800$

$-6.90859100$

$-2.55200900$

$-4.43567300$

$-1.12277800$

$-2.60386600$

$-1.71278900$

1. 02932100

$-1.25803300$

$-5.96086800$

1. 18134700

$-3.18501600$

2. 56259800

$-2.28039100$

3. 04041700

5. 48737100

4. 36655100

4. 52887900

$-5.70517000$

$-6.93864700$

$-7.68204900$

$-7.42110700$

$-6.65103500$

$-6.16339800$

$-6.90230400$

-5. 32253100

$-6.63363500$

$-5.12516900$

$-4.86472200$

$-4.22919400$

$-5.86878500$

5. 30169500

5. 70740700

4. 91771600

5. 89112900

6. 62670000

4. 02877700

3. 20363500

3. 69376500

4. 22119800

5. 12165300

6. 58149900

6. 72019100
$-3.38060800$

$-0.54778900$

0.65950800

1. 38433700

2. 31465300

2. 05443000

2. 23583400

3. 36175100

$-0.07845800$

$-0.35123800$

$-0.77638700$

$-0.23465400$

1. 50809600

2. 54263000

0. 89019300

1. 17362800

$-1.69823200$

-3. 16172800

$-3.86126900$

$-3.30959000$

$-3.43262300$

$-1.39995700$

$-2.05464000$

$-0.36499800$

$-1.56684800$

$-3.22173200$

$-3.73313900$

$-4.53982000$
-0. 78929100

$-0.31851600$

$-2.13540500$

$-1.95776700$

$-2.40431300$

$-1.20506800$

$-1.08348900$

-0. 61019400

$-2.71788000$

$-0.02139600$

0. 05113700

2. 03155500

$-0.89264500$

$-0.37195100$

2. 59543100

$-1.54855300$

0. 33151900

$-2.68666700$

1. 40476500

2. 33822100

2. 23399000

2. 99771200

1. 25388100

2. 38117400

2. 14185200

2. 90460700

2. 23383000

1. 16501700

3. 76044900

4. 00889500

3. 88963700

4. 49248500

2. 54395500

2. 80195700

2. 50524000

3. 87229600

2. 27001100

3. 37152700

3. 06971600

3. 24953900

4. 43869500

$-2.35396800$

$-2.32231000$

$-3.05281300$ 
$6.85578900 \quad-4.12527900 \quad-1.33731300$

7. 28374900

$-2.92809900$

$-2.56796900$

$4.16053000-4.38986700$

$-2.03043300$

4. 37994200

$-4.83788500$

$-1.05506700$

4. 25218600

$-5.18117900$

$-2.78475900$

3. 12010700

$-4.04695300$

$-2.01625100$

4. 81646200

$-2.73867900$

$-3.78497900$

5. 48476800

$-1.92649000$

$-4.09286000$

3. 78312900

$-2.38878000$

$-3.88582200$

4. 95663300

$-3.56584600$

$-4.49014900$

$-4.59527700$

1. 90077100

$-2.61412600$

$-4.92845800$

0. 42454400

$-2.93072400$

$-4.05247500$

$-0.21983000$

$-2.80032000$

$-5.26332900$

0. 33017900

$-3.97070400$

$-5.72903600$

0. 03280500

$-2.29480400$

$-3.50522000$

2. 36173700

$-3.60118900$

$-2.57549000$

1. 79567800

$-3.47626300$

-3. 27483400

3. 42722100

$-3.48993600$

$-3.85319400$

2. 20766500

$-4.62876800$

$-5.85636400$

2. 76746300

$-2.84933800$

$-6.68916400$

2. 45608600

$-2.20962400$

$-6.18688200$

2. 68343900

$-3.89193600$

$-5.65142100$

3. 82394200

$-2.64152500$

6. 45499500

$-0.78962600$

3. 03380400

6. 21215500

0. 27211500

2. 92255900

7. 37364700

$-0.98298000$

2. 46780300

6. 66297000

$-0.97554300$

4. 09483200

$-1.58966000$

$-3.96194700$

$-2.23427300$

$-1.28374600$

$-4.90035200$

$-1.75180200$

$-1.81175000$

$-4.20118500$

$-3.28241700$

$-0.72185100$

$-3.29578500$

$-2.22436800$ 
TS-III-R (4ad) (Table S8, entry 9)

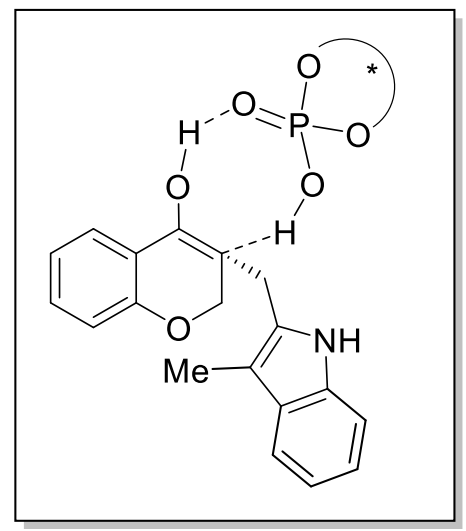

SCF energy [B3LYP-GD3BJ/6-311+G**/SMD (Chlorobenzene)]: -3331.002620810 a.u.

Thermal correction to Gibbs free energy at $258 \mathrm{~K}: 1.137063000$ a.u. Gibbs free energy at $258 \mathrm{~K}$ [B3LYP-GD3BJ/6-311+G**/SMD (Chlorobenzene)]: -3329.865557810 a.u.

01

$\mathrm{P}$

0

0.76662700

1. 31857800

0. 01728300

0.00722400

2. 57854300

$-0.70274500$

0

2. 22533000

1. 92244600

0. 43293600

0

0.94450700

0. 27401000

$-1.10601200$

0

0. 06621700

0.87236200

1. 26938100

C

$-0.19597700$

3. 71979600

0. 08794800

C

$-1.38395500$

3. 86493300

0. 82855700

C

$-1.49163200$

4. 97290100

1. 68529100

-0. 46171400

5. 90151800

1. 82314000

C

0. 68919200

5. 74691200

1. 05921100

1. 94438900

6. 59083100

1. 06198800

2. 96421100

5. 66453800

0. 36775000

2. 11135100

4. 80634500

$-0.62834400$

0. 81617300

4. 67509500

0. 16209200

1. 90541000

5. 59578800

$-1.96313200$

3. 20392600

5. 35106200

$-2.76365200$

3. 63485100

3. 98315300

$-2.28013000$

4. 58627300

3. 12537200

$-2.81967000$

C

4. 81611400

1. 89713500

$-2.20351200$

4. 06565600

1. 45861900

$-1.09675000$

3. 08084200

2. 33806700

$-0.59986300$

2. 91582500

3. 61435700

$-1.13327500$

0. 40844900

$-0.80413900$

$-0.83172000$

H

$-2.40679300$

5. 08939800

2. 25871700

$-0.56023500$

6. 72947700

2. 52079400 


\begin{tabular}{|c|c|c|c|}
\hline $\mathrm{H}$ & 1. 79900000 & 7. 52174700 & 0.49539100 \\
\hline H & 2. 25485300 & 6. 88210500 & 2. 07189100 \\
\hline & 3. 77402100 & 6. 20398800 & -0.13341300 \\
\hline & 3. 41723100 & 4. 99524100 & 1. 10788000 \\
\hline & 1. 69210600 & 6. 65586400 & -1.79231800 \\
\hline & 1. 05231200 & 5. 16499400 & -2.49946500 \\
\hline & 3. 96367100 & 6. 11251500 & -2.53731500 \\
\hline & 3. 04339600 & 5. 38135700 & -3.84757600 \\
\hline & 5. 15659400 & 3. 40873200 & -3.70091700 \\
\hline & 5. 59658300 & 1. 24728200 & -2.58668100 \\
\hline & 4. 36413100 & 0.12899200 & -0.49941300 \\
\hline & 4. 71682300 & -0.94363700 & -1.33220600 \\
\hline & 4. 39483100 & -0.05472100 & 0.89100600 \\
\hline & 5. 12223100 & -2.17561400 & -0.80971600 \\
\hline & 4. 77571600 & -1.27683500 & 1. 45475500 \\
\hline & 5. 14065100 & -2.31488900 & 0.58521900 \\
\hline & -2.53475300 & 2. 92030500 & 0.75035000 \\
\hline & -3.24433200 & 2. 74987000 & -0.43945200 \\
\hline & -2.99110500 & 2. 28972600 & 1. 91856600 \\
\hline & -4.41628800 & 1.97599600 & -0.48696500 \\
\hline & -4.13865200 & 1. 49507200 & 1.91285100 \\
\hline & -4.83073200 & 1. 35137800 & 0.69518700 \\
\hline & -0.09034000 & -2.98150600 & -1.68289200 \\
\hline & 0.83884900 & -4.29303600 & 0.05853800 \\
\hline & 0.17565300 & -3.53141900 & 1. 04628400 \\
\hline & -0.47390300 & -1.87956300 & -0.69941900 \\
\hline & 1. 95924700 & -6.06771100 & -0.37567900 \\
\hline & -0.96032300 & -3.60919000 & -1.92610700 \\
\hline & 1. 45180900 & -5.50312100 & 0.39879600 \\
\hline & 0.14666100 & -4.00018400 & 2.37447800 \\
\hline$C$ & 0.73565500 & -5.21110000 & 2. 70529700 \\
\hline & 1. 39100600 & -5.95595600 & 1. 71251400 \\
\hline & -0.34820800 & -3.39473300 & 3. 12583100 \\
\hline & 0.69750100 & -5.57676400 & 3. 72659100 \\
\hline & 1. 85719300 & -6.90353700 & 1.96893100 \\
\hline & -0.81941800 & -1.48913100 & 1. 64917500 \\
\hline o & 0.96128500 & -3.84781400 & -1.21300000 \\
\hline 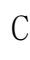 & -1.72371400 & -1.08441600 & -1.16939500 \\
\hline & -1.49634500 & -0.62542600 & -2.13531500 \\
\hline & -1.90885200 & -0.25194400 & -0.48259300 \\
\hline & -3.58900500 & -2.46389100 & -2.39637900 \\
\hline & -4.70387600 & -3.23687600 & -1.90388900 \\
\hline & -2.95268900 & -1.93627300 & -1.29025200 \\
\hline & -4.68746400 & -3.14834800 & -0.48531000 \\
\hline
\end{tabular}


$-3.60192700$

$-5.70894900$

$-6.66231500$

$-7.44504200$

$-6.63123700$

$-7.38948300$

$-5.64487800$

$-5.74166200$

$-5.61749900$

$-3.40412900$

0. 29368100

$-0.64625100$

$-2.89347400$

$-5.72862600$

$-2.41842300$

4. 12766700

5. 45019600

4. 65979100

$-5.23311900$

$-5.77783300$

-6. 42083000

-6. 36999200

$-4.96761400$

-6. 43040100

$-7.14846600$

-6. 11337000

$-6.96369700$

$-4.33918200$

$-3.97485000$

$-3.47221600$

$-4.91241700$

4. 82245200

5. 55127300

6. 27117300

6. 64546600

6. 32015200

6. 95237800

3. 89328900

4. 18613200

2. 85618300

3. 92441100

4. 36109700

3. 33379700

5. 00963300
$-2.37042800$

$-3.99717500$

$-4.63078000$

$-5.21972500$

$-4.52215400$

$-5.02796100$

$-3.78229000$

$-4.08768600$

$-3.70528700$

$-2.02065400$

$-2.55305600$

-0. 49682600

3. 26375600

0. 74494600

2. 43228400

0. 77737200

$-3.26512200$

$-0.80088700$

1. 91055000

3. 32631000

3. 68619900

3. 31324800

4. 05156900

0. 94544300

1. 25989500

$-0.07919800$

0. 92452800

1. 44298600

0. 42565600

2. 09560100

1. 44223400

$-1.50312900$

$-3.35391200$

$-1.83782700$

$-2.73899300$

$-2.00898200$

$-1.01471600$

$-2.68124700$

$-3.61060000$

$-2.46704100$

$-2.86030600$

$-0.26417600$

0. 02166500

0. 60079300
-0. 14073800

$-2.52563600$

$-1.73697000$

$-2.20743800$

$-0.33212900$

0. 25956700

0. 31274400

$-3.60856200$

1. 39647100

0. 78536300

$-2.61188000$

1. 45314900

$-1.32909900$

0. 67866100

2. 82687800

1. 52767900

1. 01080600

$-2.40437800$

$-1.79338400$

$-2.10446000$

$-1.29297200$

$-3.02802900$

$-2.23505500$

$-1.69523900$

$-0.92927600$

$-1.47176700$

$-2.65253800$

$-2.96447700$

$-2.78905500$

$-3.10882600$

$-3.89987100$

2. 97896200

$-1.70614200$

3. 40687400

2. 90933000

4. 48951000

3. 16211800

3. 35472200

2. 85521000

3. 07484000

4. 43691800

3. 77084400

3. 51929200

3. 59088100 
$\begin{array}{lll}7.33352600 & -4.58517000 & -2.00293000\end{array}$

7. $12576100-4.04870400$

$-0.32909400$

$7.69208800-2.90516800$

$-1.55483200$

5. $48055000-3.00863700$

$-3.20609700$

6. 13965100

$-2.17198000$

$-3.46474600$

4. 46203000

$-2.75161100$

$-3.51785900$

5. 79877000

$-3.87416900$

$-3.79845000$

4. 62411900

$-4.56485700$

$-1.45230600$

4. 66086500

$-4.89179900$

$-0.40761900$

4. 92539700

$-5.41405800$

$-2.07906800$

3. 58457200

$-4.31326400$

$-1.68789000$

$-0.40349300$

$-2.25932500$

0. 65782900

$-4.66329000$

0. 79766500

3. 18415300

$-4.60289000$

$-0.73646300$

2. 99520700

-5. 19499700

$-1.05969800$

2. 13190600

$-4.99722800$

$-1.25003800$

3. 88105400

$-3.56476700$

$-1.06460600$

2. 85448700

$-3.83554400$

1. 15500200

4. 43391000

$-3.85124300$

2. 23187600

4. 63599800

$-2.79044700$

0. 84097900

4. 33775300

-4. 25403800

0. 64735800

5. 31053400

-6. 12833100

1. 22026400

3. 44677800

$-6.50865700$

0. 73254500

4. 35277800

$-6.79129200$

0. 94588700

2. 61997900

-6. 20212000

2. 30447800

3. 58836400

$-3.21455000$

$-2.30545200$

$-3.84063500$

$-2.95168800$

$-3.26829100$

$-4.29925800$

$-4.04490100$

$-1.89011600$

-4. 42623500

$-2.35818900$

$-1.63643700$

$-3.97133700$ 
TS-III-R2 (Table S9, entry 1)

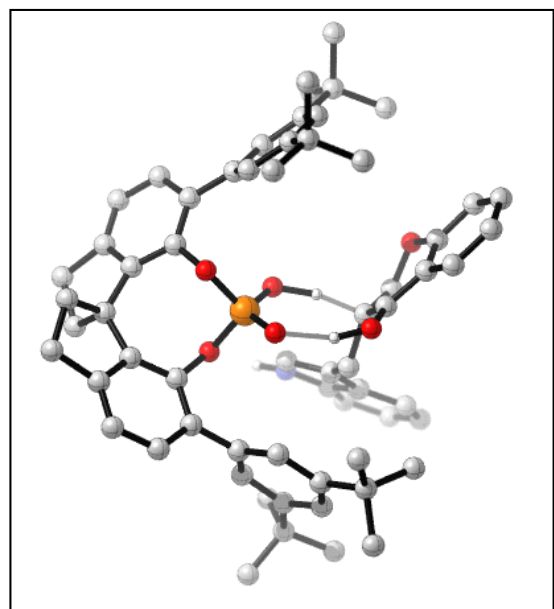

SCF energy [B3LYP-GD3BJ/6-311+G**/SMD (Chlorobenzene)]: -3291.663367400 a.u.

Thermal correction to Gibbs free energy at $258 \mathrm{~K}: 1.110230000$ a.u.

Gibbs free energy at $258 \mathrm{~K}$ [B3LYP-GD3BJ/6-311+G**/SMD (Chlorobenzene)]: -3290.553137400 a.u.

01

$\begin{array}{lrrr}\mathrm{P} & 0.17801400 & 1.20661200 & 0.29432000 \\ 0 & -1.09128100 & 1.97204000 & -0.41382600 \\ 0 & 1.30385500 & 2.37291600 & 0.45701200 \\ 0 & 0.64354300 & 0.19098400 & -0.77567200 \\ 0 & -0.16387700 & 0.68176000 & 1.65672100 \\ \mathrm{C} & -1.67889400 & 3.01206400 & 0.32456000 \\ \mathrm{C} & -2.77203600 & 2.74921500 & 1.17006500 \\ \mathrm{C} & -3.24878800 & 3.79893900 & 1.97134000 \\ \mathrm{C} & -2.66155100 & 5.06333300 & 1.95585200 \\ \mathrm{C} & -1.60436800 & 5.30307100 & 1.08576000 \\ \mathrm{C} & -0.79431400 & 6.56897000 & 0.91122600 \\ \mathrm{C} & 0.44794000 & 6.06332400 & 0.14892900 \\ \mathrm{C} & -0.07172900 & 4.85658000 & -0.70414900 \\ \mathrm{C} & -1.12942300 & 4.29091500 & 0.23725800 \\ \mathrm{C} & -0.70340400 & 5.38516600 & -2.03483300 \\ \mathrm{C} & 0.50110400 & 5.60915400 & -2.97564100 \\ \mathrm{C} & 1.48586800 & 4.56277300 & -2.50025500 \\ \mathrm{C} & 2.63703700 & 4.09665200 & -3.12536600 \\ \mathrm{C} & 3.39216200 & 3.10497800 & -2.50195600 \\ \mathrm{C} & 2.99479300 & 2.50840300 & -1.29123500 \\ \mathrm{C} & 1.80592800 & 2.98492600 & -0.70094100 \\ \mathrm{C} & 1.09240300 & 4.04315300 & -1.25788600 \\ \mathrm{H} & 0.50199700 & -0.97311000 & -0.46334300\end{array}$




\begin{tabular}{|c|c|c|c|}
\hline $\mathrm{H}$ & -4.08869600 & 3. 59846200 & 2. 63039200 \\
\hline H & -3.02656000 & 5. 84501700 & 2.61746000 \\
\hline & -1.34657500 & 7. 31723800 & 0.32500800 \\
\hline & -0.54118400 & 7. 04226300 & 1. 86676000 \\
\hline & 0.92727100 & 6. 83195600 & -0.46549200 \\
\hline & 1. 19331800 & 5. 69369700 & 0.86214400 \\
\hline & -1.30983700 & 6. 28341500 & -1.88109200 \\
\hline & -1.35686200 & 4. 60770700 & -2.44715400 \\
\hline & 0.91107300 & 6. 62313300 & -2.86507600 \\
\hline & 0.23950100 & 5. 49100300 & $-4.0336230 \mathrm{c}$ \\
\hline & 2. 95516600 & 4. 50382700 & -4.08205600 \\
\hline & 4. 31696200 & 2. 76981600 & -2.96119300 \\
\hline & 3. 83263600 & 1. 43288300 & $-0.6958730 c$ \\
\hline & 4. 44041900 & 0.48429000 & -1.53132800 \\
\hline & 4. 08754800 & 1. 38593100 & 0.68242200 \\
\hline & 5. 29965400 & -0.49511700 & -1.02374900 \\
\hline & 4. 92564200 & 0.41129100 & 1. 23375300 \\
\hline & 5. 51864000 & -0.51275500 & 0.36106000 \\
\hline & -3.47056800 & 1. 42858000 & 1. 25099100 \\
\hline & -4.37015600 & 1. 05173600 & 0.25235500 \\
\hline & -3.32839600 & 0.62358400 & 2. 39179600 \\
\hline & -5.14339000 & -0.11549300 & 0.36777300 \\
\hline & -4.06740800 & -0.55283700 & 2.54045600 \\
\hline & -4.96580900 & -0.89744400 & 1. 51397700 \\
\hline & 0.52258200 & -3.30615900 & -1.16669500 \\
\hline & 2. 17613500 & -4.08082100 & 0.33528800 \\
\hline & 1. 51158100 & -3.48830700 & 1. 42970900 \\
\hline & 0.01307400 & -2.28487800 & -0.15522900 \\
\hline & 3. 70572300 & -5.43702300 & -0.30751800 \\
\hline & -0.11197900 & -4.20769400 & -1.17059500 \\
\hline & 3. 20621900 & -5.00128900 & 0.55106600 \\
\hline & 1.89787100 & -3.83307200 & 2. 73849800 \\
\hline & 2. 90529200 & -4.76344300 & 2. 95203500 \\
\hline & 3. 56009100 & -5.33978600 & 1. 85355800 \\
\hline & 1. 38996000 & -3.35840200 & 3.57073200 \\
\hline & 3. 19014200 & -5.03713700 & 3. 96317200 \\
\hline & 4. 35298200 & -6.06488600 & 2. 01696300 \\
\hline & 0.06427600 & -1.81845100 & 2.20848400 \\
\hline & 1. 88296300 & -3.72607500 & -0.93795600 \\
\hline & -1.49979700 & -1.95538300 & -0.34164200 \\
\hline & -1.75325500 & -1.03366200 & 0.18820000 \\
\hline & -2.08248700 & -2.74753800 & 0.14776700 \\
\hline & -1.93606300 & -1.86410500 & -1.78052500 \\
\hline & -2.57688700 & -2.90556700 & -2.5507090 \\
\hline
\end{tabular}


$-1.79421600$

$-0.78971100$

$-2.79149800$

$-2.39191800$

$-2.30080700$

$-1.10258300$

$-3.00156700$

-4. 21666400

$-3.60786200$

$-4.96685200$

$-3.93812600$

$-5.97978700$

$-3.80369100$

$-4.43624600$

$-4.28006600$

$-5.04682900$

$-3.40029900$

$-3.14365100$

$-2.86030900$

$-4.63920600$

$-2.73274100$

$-3.55404500$

$-2.32826800$

0. 51971600

$-0.47317000$

$-2.89271100$

$-1.36249500$

$-0.23820200$

0. 18069500

$-0.87851100$

$-4.48777900$

1. 70082500

$-5.55064100$

$-2.61892900$

3. 62384800

6. 17305000

4. 21512000

-6. 20427600

$-7.38053200$

$-7.83098900$

$-8.16068400$

$-7.04881300$

$-6.75456600$

$-7.28774500$

$-5.95515400$

$-7.46676400$

$-5.61420500$

$-4.77441800$

$-5.26164200$

$-6.38144800$

5. 21029700

5. 99488600

6. 72954700

7. 30556500

6. 94664200

7. 09514400

4. 73022800

5. 23403600

3. 65256000

4. 93271900

$-1.80292500$

0. 93473000

2. 12741100

$-1.27431700$

0. 51348900

$-0.44878800$

0. 54626000

0. 47004500

0. 33926700

1. 58136400

$-1.88154000$

$-2.02778600$

$-2.62827600$

$-2.08498800$

$-0.31693500$

$-1.00320300$

0. 69818100

$-0.55726200$

0. 33026900

$-1.53387800$

0. 47773900

$-0.30856400$

0. 41857800

1. 44374300

$-1.03845500$

$-1.87389500$

$-1.16239700$

$-1.11808200$

$-2.62678000$

$-3.86098600$

$-3.87248700$

$-2.26524400$

$-3.26526600$

$-3.05149500$

$-4.55694800$

$-5.31907600$

$-4.87120200$

$-1.27331900$

$-5.86588100$

$-4.65928800$

$-2.17500700$

$-2.43050800$

1. 96992400

$-0.60982400$

1. 62498500

3. 14806200

1. 31861800

0. 77531200

$-2.59041300$

$-0.70080200$

$-0.54578000$

0. 45062000

$-1.28940200$

$-0.68394700$

$-0.55576700$

0. 39036300

$-0.62161100$

$-1.36349000$

$-2.12250200$

$-2.26388400$

$-2.33204200$

$-2.86871100$

2. 74653700

$-1.92599000$

2. 99816200

2. 49888300

4. 07203000

2. 63098200

3. 28447900

2. 78721400

3. 13084300

4. 36006100 


$\begin{array}{lrrr}\text { C } & 4.48487600 & 1.43394900 & 3.54011200 \\ \mathrm{H} & 3.39723300 & 1.37247100 & 3.42446300 \\ \mathrm{H} & 4.80589900 & 2.43562500 & 3.23184500 \\ \mathrm{H} & 4.71191900 & 1.32893000 & 4.60726200 \\ \mathrm{C} & 7.52817900 & -1.42886400 & -1.74737900 \\ \mathrm{H} & 8.03748600 & -2.16378700 & -2.38321100 \\ \mathrm{H} & 7.83411300 & -1.61664500 & -0.71291200 \\ \mathrm{H} & 7.88838400 & -0.43146500 & -2.02508000 \\ \mathrm{C} & 5.67615500 & -1.32326100 & -3.41865200 \\ \mathrm{H} & 6.00010100 & -0.33681100 & -3.77023000 \\ \mathrm{H} & 4.60515600 & -1.42604100 & -3.62563300 \\ \mathrm{H} & 6.20141300 & -2.07656700 & -4.01690600 \\ \mathrm{C} & 5.52712500 & -2.95420100 & -1.53304900 \\ \mathrm{H} & 5.76163000 & -3.18324200 & -0.48812000 \\ \mathrm{H} & 6.02254600 & -3.70646100 & -2.16049300 \\ \mathrm{H} & 4.44449300 & -3.05782200 & -1.66255700 \\ \mathrm{C} & 0.48266700 & -2.49604700 & 1.15463700 \\ \mathrm{C} & -3.94595700 & -1.45801400 & 3.78296400 \\ \mathrm{C} & -3.49983300 & -2.87609100 & 3.35476900 \\ \mathrm{H} & -4.19812900 & -3.32721200 & 2.64163900 \\ \mathrm{H} & -3.44269900 & -3.53741800 & 4.22853600 \\ \mathrm{H} & -2.50881900 & -2.84542700 & 2.88900900 \\ \mathrm{C} & -2.91799300 & -0.92497500 & 4.79944100 \\ \mathrm{H} & -3.19101500 & 0.06798400 & 5.17423100 \\ \mathrm{H} & -1.91250700 & -0.86460100 & 4.36919900 \\ \mathrm{H} & -2.86902800 & -1.60056100 & 5.66133400 \\ \mathrm{C} & -5.31999300 & -1.54596900 & 4.48964600 \\ \mathrm{H} & -5.25126500 & -2.18436200 & 5.37918000 \\ \mathrm{H} & -6.09027000 & -1.96838300 & 3.83576500 \\ \mathrm{H} & -5.66024000 & -0.55414700 & 4.80904100\end{array}$


TS-III-R3 (Table S9, entry 2)

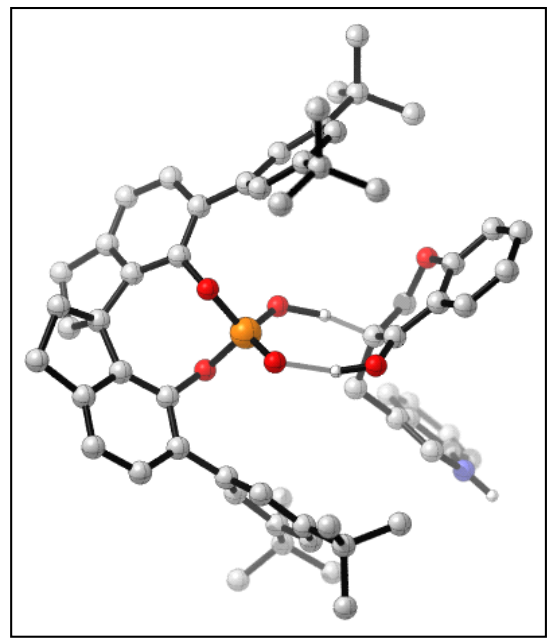

SCF energy [B3LYP-GD3BJ/6-311+G**/SMD (Chlorobenzene)]: -3291.662406070 a.u.

Thermal correction to Gibbs free energy at $258 \mathrm{~K}: 1.109813000$ a.u.

Gibbs free energy at $258 \mathrm{~K}$ [B3LYP-GD3BJ/6-311+G**/SMD (Chlorobenzene)]: -3290.552593070 a.u.

01

$\mathrm{P}$

0

0.33928100

1. 33379500

0.04536000

0

$-0.71502800$

2. 32323800

$-0.72712300$

0

1. 65120500

2. 27626200

0. 28926000

0. 68191000

0. 24126300

$-0.99001000$

0

$-0.17407000$

0. 88590000

1. 38371100

C

$-1.12145400$

3. 47008400

$-0.03006300$

C

$-2.27364400$

3. 43500300

0. 77705600

C

$-2.58036900$

4. 57614900

1. 53593200

$-1.77418000$

5. 71278100

1. 51582600

$-0.65729800$

5. 72936600

0. 68866100

C

0. 38020100

6. 81815100

0. 52719700

1. 54272000

6. 06563300

$-0.15250600$

0. 85024100

4. 94769400

$-1.00544000$

C

$-0.34212000$

4. 62266500

$-0.11508100$

0. 40754500

5. 53584300

$-2.38574700$

C

1. 68766500

5. 50141800

$-3.24962200$

C

2. 43211500

4. 31167700

$-2.68365400$

3. 51864800

3. 62798700

$-3.21634300$

C

4. 04150000

2. 54160200

$-2.51826500$

C

3. 46275300

2. 06308300

$-1.32817600$

C

2. 33772700

2. 75798600

$-0.83549200$

C

1. 87086600

3. 91176600

$-1.46151200$

H

0. 39181100

$-0.89634000$

$-0.60221500$ 


\begin{tabular}{|c|c|c|c|}
\hline $\mathrm{H}$ & -3.46782300 & 4. 55130000 & 2. 16195100 \\
\hline H & -2.01643300 & 6. 56795600 & 2.14196100 \\
\hline & 0.00592300 & 7. 63366900 & -0.10810200 \\
\hline & 0.66901700 & 7. 27058600 & 1. 48276300 \\
\hline & 2. 18811300 & 6. 71172800 & -0.75591800 \\
\hline & 2. 16821600 & 5. 58791400 & 0.61000000 \\
\hline & -0.02923900 & 6. 53541700 & -2.29306600 \\
\hline & -0.35233300 & 4. 87720600 & -2.82137100 \\
\hline & 2. 26956100 & 6. 42738000 & -3.13849600 \\
\hline & 1. 47317000 & 5. 39208600 & -4.31901400 \\
\hline & 3. 96739400 & 3. 93956900 & -4.15642400 \\
\hline & 4. 92330800 & 2. 03709300 & -2.90041800 \\
\hline & 4. 07237100 & 0.88871000 & $-0.6475430 c$ \\
\hline & 4. 60518400 & -0.15894100 & -1.41374000 \\
\hline & 4. 21165800 & 0.84560000 & 0.74760500 \\
\hline & 5. 29082800 & -1.22454000 & -0.82302600 \\
\hline & 4. 87348900 & -0.21189600 & 1. 38037900 \\
\hline & 5. 40856900 & -1.22702600 & 0.57409200 \\
\hline & -3.18685700 & 2. 25945900 & 0.87022100 \\
\hline & -3.94769000 & 1. 85326100 & -0.22668000 \\
\hline & -3.37298800 & 1.62721100 & 2.10892900 \\
\hline & -4.91079700 & 0.83679800 & -0.11127000 \\
\hline & -4.30369700 & 0.59856200 & 2.26425900 \\
\hline & -5.06000400 & 0.22369100 & 1. 13799300 \\
\hline & 0.28400200 & -3.19064600 & -1.26686700 \\
\hline & 1. 61739700 & -4.13276500 & 0.44584400 \\
\hline & 0.89507200 & -3.44507600 & 1. 44494600 \\
\hline & -0.24344800 & -2.11261000 & -0.32486900 \\
\hline & 3. 04011600 & -5.67606600 & 0.01264500 \\
\hline & -0.44136800 & -4.00930400 & -1.37294100 \\
\hline & 2. 49198100 & -5.16616200 & 0.79731500 \\
\hline & 1. 07131200 & -3.80521700 & 2. 79454400 \\
\hline & 1.92183600 & -4.84553000 & 3. 13959100 \\
\hline & 2. 63375700 & -5.51892500 & 2. 13547300 \\
\hline & 0.52581300 & -3.25354100 & 3.55227300 \\
\hline & 2. 04301400 & -5.13023600 & 4. 18012000 \\
\hline & 3. 30524100 & -6.33091600 & 2. 40179900 \\
\hline & -0.40816800 & -1.58319600 & 2. 02016200 \\
\hline & 1. 54016400 & -3.76890200 & -0.85507400 \\
\hline & -1.66456400 & -1.61311300 & -0.71197500 \\
\hline & -1.58390100 & -1.01910300 & -1.62819800 \\
\hline & -2.01676000 & -0.92059500 & 0.05746200 \\
\hline & -2.67080900 & -2.71803500 & -0.91566000 \\
\hline & -3.06607000 & -3.31685700 & $-2.1706350 c$ \\
\hline
\end{tabular}


$-3.38436600$

-3. 37056700

-4. 32602100

$-4.02542000$

-4. 33524100

$-2.70938100$

$-3.09820400$

$-3.30015400$

$-3.87049400$

$-3.03077200$

$-3.70656800$

$-4.24722600$

$-4.86584900$

$-5.45422500$

$-4.69224900$

$-4.62299500$

$-5.10639300$

$-2.33156200$

$-5.87246500$

$-5.35416600$

$-4.82759900$

$-4.93442200$

$-2.77754400$

0. 47815200

$-3.38059000$

$-3.21892700$

$-0.51193100$

$-0.60709500$

$-3.80576100$

2. 36672200

$-5.79520600$

$-0.56461700$

$-2.76022000$

1. 95317700

3. 80396700

1. 65910500

5. 93637800

4. 46508200

$-5.81109300$

$-6.73655500$

$-7.36398300$

$-7.39615800$

-6. 16034400

$-6.69564900$

$-7.39965500$

$-6.09354400$

$-7.28720600$

$-4.95785500$

-4. 31945800

$-4.31733400$

$-5.61021300$

5. 04345500

5. 91846900

6. 54943400

7. 08502100

6. 68777200

7. 02304600

4. 40901500

4. 87083600

3. 33792000

4. 53032700
$-2.04787600$

$-0.13256300$

0. 49719300

1. 70773000

1. 92882600

1. 49930800

2. 60951600

$-0.73858400$

$-0.57729600$

$-1.62563200$

$-0.95811100$

0. 21670300

$-0.66027900$

1. 06433100

0. 01776700

$-0.27835200$

$-2.36442800$

$-0.25869600$

$-1.10239500$

$-0.31483700$

0. 66371500

$-1.58413800$

$-2.47460800$

$-1.61991200$

$-1.64840000$
0. 06049700

$-1.87666500$

$-0.50741200$

$-3.51433100$

$-4.50615700$

$-5.54591100$

$-4.18811200$

$-4.98577700$

$-2.87183400$

$-3.77543100$

$-2.62630300$

$-0.00027300$

$-2.25949500$

1. 13057500

1. 73420800

$-1.17270900$

1. 24944000

2. 94027000

1. 33184400

1. 05097800

$-2.48741700$

$-1.31628200$

$-1.59201500$

-0. 72071800

$-2.44378800$

$-1.82546300$

$-1.06129500$

$-0.23675900$

$-0.83474000$

$-1.95736700$

$-2.57426200$

$-2.42908200$

$-2.83736400$

$-3.43340000$

2. 91112400

$-1.64982500$

3. 26480400

2. 81660400

4. 35185000

2. 90931400

3. 44480000

3. 00560900

3. 21832200

4. 53360800 


$\begin{array}{lrrr}\mathrm{C} & 4.36647000 & 0.90548800 & 3.62843600 \\ \mathrm{H} & 3.28992300 & 0.94267400 & 3.42834900 \\ \mathrm{H} & 4.80243800 & 1.86596800 & 3.33110700 \\ \mathrm{H} & 4.50102100 & 0.80463500 & 4.71164700 \\ \mathrm{C} & 7.44380900 & -2.40322600 & -1.39280700 \\ \mathrm{H} & 7.90862100 & -3.21258500 & -1.96974600 \\ \mathrm{H} & 7.67559400 & -2.57183300 & -0.33599700 \\ \mathrm{H} & 7.91489300 & -1.45944200 & -1.69096600 \\ \mathrm{C} & 5.69616600 & -2.18497500 & -3.16390900 \\ \mathrm{H} & 6.14483700 & -1.25661300 & -3.53559500 \\ \mathrm{H} & 4.63112700 & -2.17782800 & -3.42131300 \\ \mathrm{H} & 6.16155800 & -3.01655100 & -3.70550200 \\ \mathrm{C} & 5.29184300 & -3.71500200 & -1.23376100 \\ \mathrm{H} & 5.44392900 & -3.92213600 & -0.16917200 \\ \mathrm{H} & 5.74463800 & -4.53702900 & -1.80302400 \\ \mathrm{H} & 4.21334300 & -3.71665400 & -1.42420000 \\ \mathrm{C} & 0.03595000 & -2.34483300 & 1.03830600 \\ \mathrm{C} & -4.52822500 & -0.11084000 & 3.61465700 \\ \mathrm{C} & -4.19239800 & -1.61397800 & 3.47252700 \\ \mathrm{H} & -4.82170300 & -2.09654000 & 2.71655500 \\ \mathrm{H} & -4.35410600 & -2.13574200 & 4.42428000 \\ \mathrm{H} & -3.14319200 & -1.75056900 & 3.18425500 \\ \mathrm{C} & -3.64189700 & 0.46730100 & 4.73484900 \\ \mathrm{H} & -3.84702300 & 1.52962000 & 4.90867500 \\ \mathrm{H} & -2.57558600 & 0.35799200 & 4.50878200 \\ \mathrm{H} & -3.83892600 & -0.06509100 & 5.67256600 \\ \mathrm{C} & -6.00626100 & 0.04482200 & 4.04444800 \\ \mathrm{H} & -6.17940300 & -0.45352600 & 5.00645500 \\ \mathrm{H} & -6.69441100 & -0.39382000 & 3.31420800 \\ \mathrm{H} & -6.27047300 & 1.10240900 & 4.15794100 \\ & & & \\ & & & \end{array}$


TS-III-R4 (Table S9, entry 3)

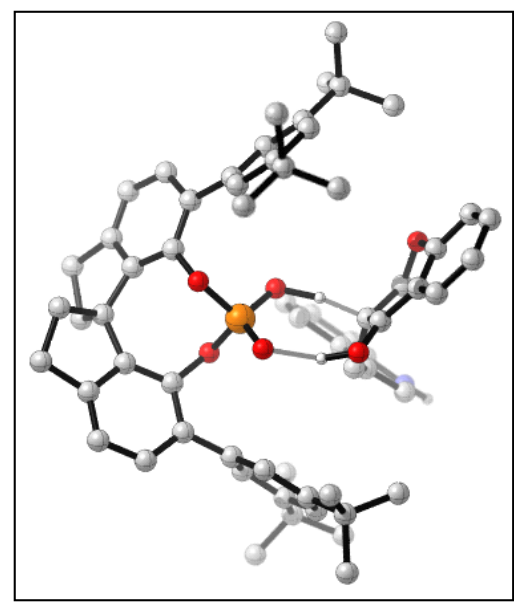

SCF energy [B3LYP-GD3BJ/6-311+G**/SMD (Chlorobenzene)]: -3291.661073750 a.u.

Thermal correction to Gibbs free energy at $258 \mathrm{~K}: 1.111165000$ a.u.

Gibbs free energy at $258 \mathrm{~K}$ [B3LYP-GD3BJ/6-311+G**/SMD (Chlorobenzene)]: -3290.549908750 a.u.

01

$\mathrm{P}$

0

0

0

0

C

C

C

C

C

C

C

C

C

C

C

C

C

C

C

C

C

$\mathrm{H}$
$-0.01701100$

1. 27776500

$-1.06862500$

$-0.57159800$

0. 32326000

1. 94371800

3. 03385100

3. 59026700

3. 08352500

2. 02882500

1. 29684100

0. 01228600

0. 44003000

1. 47588400

1. 08070200

-0. 12292200

$-1.16195300$

$-2.34932200$

$-3.15493900$

$-2.77683700$

$-1.54976100$

$-0.78129200$

$-0.49068300$
1. 06160300

1. 82810600

2. 26430000

0. 19572200

0. 37054500

2. 73416400

2. 30432700

3. 21457900

4. 50426800

4. 91343000

6. 23736700

5. 90680800

4. 78330400

4. 04486500

5. 42981000

5. 83426900

4. 79911000

4. 48096300

3. 47019800

2. 71132400

3. 03918400

4. 11213500

$-1.01246400$
$-0.45951200$

0. 19487000

$-0.78329200$

0. 69585100

$-1.74688400$

$-0.64423700$

$-1.42213800$

$-2.33453100$

$-2.48905100$

$-1.68118300$

$-1.68364300$

$-0.89476400$

0. 11049200

$-0.72772400$

1. 38363600

2. 26276200

1. 89041600

2. 53961000

2. 01922200

0. 89642700

0. 28273600

0. 72780900

0. 50890700 
-2. 94124700

3. 50868500

5. 17462200

$-3.23199200$

1. 88498100

7. 01867800

$-1.18140500$

1. 08967900

6. 60202200

$-2.69624900$

$-0.42515100$

6. 77393300

$-0.38979200$

$-0.74420100$

5. 49991700

$-1.57525400$

1. 74233100

6. 26669300

1. 13811600

1. 67900700

4. 67097400

1. 90052500

$-0.46854500$

6. 85038000

2. 02519100

0. 11379300

5. 82592100

3. 33303700

$-2.65620600$

5. 01681200

3. 43453700

$-4.10678100$

3. 24921200

2. 49214800

$-3.67992700$

1. 63409500

0. 40913400

$-4.36533900$

0. 83244800

1. 33379400

$-3.92474000$

1. 45218800

$-0.95976400$

$-5.29653500$

$-0.12609000$

0. 92281600

$-4.83610200$

0. 49323400

$-1.41417800$

$-5.51056900$

$-0.27567500$

$-0.45471400$

3. 64739800

0. 94580500

$-1.31366700$

4. 45330500

0. 62891100

$-0.21886300$

3. 52753600

0. 03082200

$-2.37049000$

5. 15825800

$-0.58451800$

-0. 15649600

4. 20019900

$-1.19323400$

$-2.34382200$

5. 00835900

$-1.47384400$

$-1.22650400$

$-0.75087400$

$-3.23391300$

1. 42520300

$-2.39797500$

$-3.97201800$

$-0.09797600$

$-1.60471300$

$-3.57933300$

$-1.19679900$

$-0.08209000$

$-2.36521600$

0. 36115000

$-4.10948000$

$-5.07779700$

0. 56740800

$-0.23153100$

$-4.19861400$

1. 53245400

$-3.51193700$

$-4.79467700$

$-0.29232400$

$-1.94976700$

$-4.01885900$

$-2.48872200$

$-3.04198600$

$-4.85360700$

$-2.67792100$

$-3.82275700$

$-5.23319900$

$-1.57574100$

$-1.34366900$

$-3.69129700$

-3. 32621200

-3. 29663700

$-5.20258800$

$-3.67381000$

$-4.68243600$

$-5.88203000$

$-1.72116800$

0. 06484000

$-2.15812600$

$-2.03009500$

$-2.13944200$

-3. 51227700

1. 14848100

1. 45454000

-2. 14093800

0. 58184400

1. 72712600

$-1.10393600$

0. 36542500

2. 00141000

$-2.74725800$

$-0.14966300$

1. 94112600

$-2.50249500$

1. 95890500

1. 79035300

$-1.75558200$

3. 18901900 
2. $93419800-3.64995200$

3. 63459300

$1.18743600-0.52670500$

3. 51780900

1. 23410300

$-0.09058100$

4. 83611000

0. 77244000

0.85658800

5. 10142400

1. 86947300

$-0.85144800$

5. 83903300

1. 88761800

$-0.48109400$

6. 86057700

2. 47470300

$-2.06755300$

5. 54501700

0.69639600

0. 06663000

2. 75265100

2. 96644700

$-2.65445600$

6. 31676100

3. 46747700

$-4.36104800$

4. 10964200

$-0.75006300$

$-2.74157200$

2. 39732900

2. 96419300

$-4.45179000$

1. 64631600

0. 39007500

$-1.20494700$

$-1.87535000$

4. 55278500

1. 36016300

0. 57722300

5. 54485000

$-2.41545700$

$-1.20595500$

2. 88876200

0. 29844800

$-3.20291500$

$-3.39662600$

2. 07864700

$-1.66566800$

-6. 22694900

$-1.01885700$

$-0.79275200$

-4. 14583700

0. 96104600

2. 38677600

6. 12228900

$-0.85434100$

1. 01608000

7. 35804500

0. 06465300

0. 85327900

7. 87647100

$-0.13583600$

$-0.09162400$

8. 06926600

$-0.09714700$

1. 67338400

7. 07194700

1. 12211400

0. 85904300

6. 60666000

$-2.31714300$

1. 05182600

7. 19310800

$-2.58193000$

0. 16502300

5. 76538000

$-3.01558000$

1. 13048800

7. 25191100

$-2.46974200$

1. 92467500

5. 44068300

$-0.54947100$

2. 36885500

4. 55102000

$-1.16964500$

2. 51253300

5. 13300700

0. 49730100

2. 45205100

6. 13639100

$-0.75062700$

3. 19285800

$-5.12253300$

0. 27365700

$-2.91292600$

$-6.07763100$

$-1.00263000$

1. 92154500

$-6.61392300$

0. 56663400

$-3.20189700$

$-7.27737400$

$-0.08279400$

$-2.62075000$

$-6.83678200$

0. 40654200

$-4.26431800$

$-6.86380600$

1. 60509000

$-2.95539400$

$-4.80638100$

$-1.19297000$

$-3.28956200$

$-5.40984000$

$-1.90390200$

$-2.71556800$

$-3.75246600$

$-1.42661000$

$-3.10382800$

$-5.01113000$

$-1.36479200$

$-4.35384100$ 


\begin{tabular}{lrrr}
$\mathrm{C}$ & -4.26981800 & 1.18844100 & -3.81319800 \\
$\mathrm{H}$ & -3.19721600 & 1.02161500 & -3.66471300 \\
$\mathrm{H}$ & -4.47886000 & 2.24905700 & -3.63284700 \\
$\mathrm{H}$ & -4.49642800 & 0.98298100 & -4.86581100 \\
$\mathrm{C}$ & -7.59569900 & -0.77028600 & 1.73263400 \\
$\mathrm{H}$ & -8.16751600 & -1.39126900 & 2.43360000 \\
$\mathrm{H}$ & -7.92536100 & -1.02306800 & 0.71939500 \\
$\mathrm{H}$ & -7.85798000 & 0.27816800 & 1.91588400 \\
$\mathrm{C}$ & -5.72577700 & -0.68218600 & 3.38674600 \\
$\mathrm{H}$ & -5.95588200 & 0.35814400 & 3.64412200 \\
$\mathrm{H}$ & -4.66612800 & -0.86130700 & 3.59970900 \\
$\mathrm{H}$ & -6.31056900 & -1.32490800 & 4.05484500 \\
$\mathrm{C}$ & -5.74966800 & -2.49201600 & 1.66724400 \\
$\mathrm{H}$ & -6.01632200 & -2.79759300 & 0.64987400 \\
$\mathrm{H}$ & -6.30669600 & -3.13010400 & 2.36543100 \\
$\mathrm{H}$ & -4.67979400 & -2.68281300 & 1.80403100 \\
$\mathrm{C}$ & -0.49475300 & -2.67193500 & -0.95030200 \\
$\mathrm{C}$ & 4.11418400 & -2.21224800 & -3.49787400 \\
$\mathrm{C}$ & 3.59019500 & -3.56777100 & -2.96770700 \\
$\mathrm{H}$ & 4.22828100 & -3.97182800 & -2.17443700 \\
$\mathrm{H}$ & 3.55986600 & -4.30686700 & -3.77810000 \\
$\mathrm{H}$ & 2.57504700 & -3.46073400 & -2.56984700 \\
$\mathrm{C}$ & 3.16690400 & -1.74756400 & -4.62091800 \\
$\mathrm{H}$ & 3.49974900 & -0.80634300 & -5.07248300 \\
$\mathrm{H}$ & 2.14239900 & -1.61073500 & -4.25798100 \\
$\mathrm{H}$ & 3.14003900 & -2.50231100 & -5.41552000 \\
$\mathrm{C}$ & 5.52306000 & -2.41189900 & -4.10650200 \\
$\mathrm{H}$ & 5.48567900 & -3.13783300 & -4.92832600 \\
$\mathrm{H}$ & 6.23859600 & -2.78350100 & -3.36525900 \\
$\mathrm{H}$ & 5.91430700 & -1.46832300 & -4.50400500 \\
& & & \\
\hline
\end{tabular}


TS-III-S2 (Table S9, entry 4)

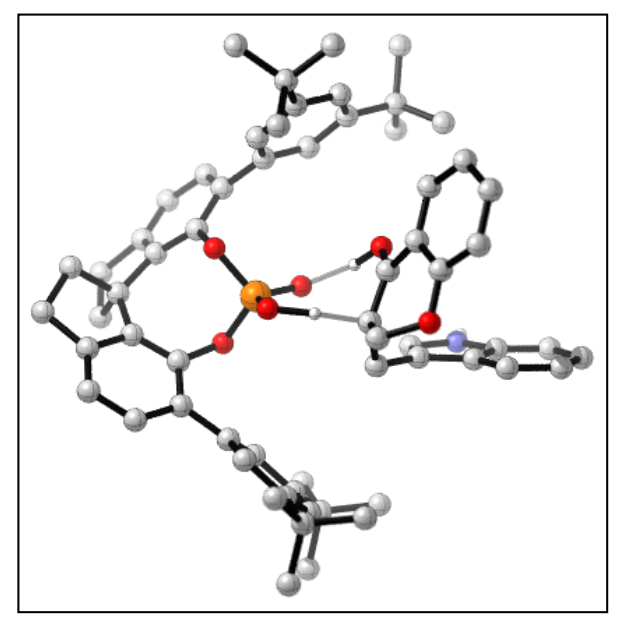

SCF energy [B3LYP-GD3BJ/6-311+G**/SMD (Chlorobenzene)]: -3291.666535210 a.u.

Thermal correction to Gibbs free energy at $258 \mathrm{~K}: 1.110519000$ a.u.

Gibbs free energy at $258 \mathrm{~K}$ [B3LYP-GD3BJ/6-311+G**/SMD (Chlorobenzene)]: -3290.556016210 a.u.

01

$\begin{array}{lrrr}\mathrm{P} & 0.02266300 & 1.37180600 & 0.02549300 \\ 0 & -1.03980700 & 2.49368900 & -0.50901100 \\ 0 & 1.25789300 & 2.25160200 & 0.64239900 \\ 0 & 0.47085000 & 0.66441000 & -1.27375200 \\ 0 & -0.49779400 & 0.50645400 & 1.13672800 \\ \mathrm{C} & -1.62098400 & 3.31859900 & 0.46318500 \\ \mathrm{C} & -2.85450500 & 2.96067400 & 1.04084400 \\ \mathrm{C} & -3.32914400 & 3.75450300 & 2.09866400 \\ \mathrm{C} & -2.61100700 & 4.84899800 & 2.58025400 \\ \mathrm{C} & -1.41797600 & 5.20148800 & 1.95829800 \\ \mathrm{C} & -0.46055200 & 6.32315100 & 2.29893700 \\ \mathrm{C} & 0.81233900 & 5.92295500 & 1.52055900 \\ \mathrm{C} & 0.28446400 & 5.14487200 & 0.26819600 \\ \mathrm{C} & -0.93750200 & 4.46129500 & 0.86756300 \\ \mathrm{C} & -0.12620000 & 6.15924200 & -0.85175500 \\ \mathrm{C} & 1.19244900 & 6.49390900 & -1.58154100 \\ \mathrm{C} & 1.99052700 & 5.21939100 & -1.41510100 \\ \mathrm{C} & 3.13631200 & 4.81485200 & -2.09070900 \\ \mathrm{C} & 3.71036700 & 3.58529900 & -1.77275300 \\ \mathrm{C} & 3.13147600 & 2.70999300 & -0.83692800 \\ \mathrm{C} & 1.94023000 & 3.12824600 & -0.20943600 \\ \mathrm{C} & 1.41013800 & 4.39649300 & -0.43780300\end{array}$




$\begin{array}{lrrr}\mathrm{H} & 0.46048900 & -0.54092300 & -1.16208100 \\ \mathrm{H} & -4.28268100 & 3.49412400 & 2.54897000 \\ \mathrm{H} & -2.98792600 & 5.41973900 & 3.42541200 \\ \mathrm{H} & -0.84802100 & 7.29414900 & 1.95892500 \\ \mathrm{H} & -0.28503000 & 6.41516900 & 3.37692900 \\ \mathrm{H} & 1.44282800 & 6.77502500 & 1.24711900 \\ \mathrm{H} & 1.41760800 & 5.24087900 & 2.12834500 \\ \mathrm{H} & -0.63835700 & 7.03901600 & -0.44939100 \\ \mathrm{H} & -0.81429500 & 5.65922600 & -1.54273300 \\ \mathrm{H} & 1.70379000 & 7.34713000 & -1.11336100 \\ \mathrm{H} & 1.03758000 & 6.75959500 & -2.63357200 \\ \mathrm{H} & 3.59111200 & 5.45069400 & -2.84628900 \\ \mathrm{H} & 4.63568300 & 3.28405500 & -2.25460200 \\ \mathrm{C} & 3.82978000 & 1.43401600 & -0.51670300 \\ \mathrm{C} & 4.38366800 & 0.65909600 & -1.54644100 \\ \mathrm{C} & 4.05863400 & 1.05940000 & 0.81595800 \\ \mathrm{C} & 5.18447500 & -0.45609300 & -1.27180700 \\ \mathrm{C} & 4.85104400 & -0.04950500 & 1.13465100 \\ \mathrm{H} & & & \end{array}$




\begin{tabular}{|c|c|c|c|}
\hline C & 0.98387500 & -2.75615400 & 1. 50799800 \\
\hline $\mathrm{C}$ & 0.86447000 & -4.19111900 & 1. 62477600 \\
\hline $\mathrm{C}$ & 0.58820000 & -2.23485600 & 2. 71652200 \\
\hline C & 0.40119600 & -4.46843800 & 2. 94128900 \\
\hline $\mathrm{N}$ & 0.24108100 & -3.25528800 & 3. 58058800 \\
\hline $\mathrm{C}$ & 1. 10603800 & -5.26688900 & 0.75220900 \\
\hline $\mathrm{C}$ & 0.89366200 & -6.56387200 & 1. 20426900 \\
\hline H & 1. 07536300 & -7.40164500 & 0.53631000 \\
\hline C & 0.44153400 & -6.81416400 & 2. 51572700 \\
\hline H & 0.28492900 & -7.83922600 & 2. 84093200 \\
\hline $\mathrm{C}$ & 0.18854900 & -5.77181400 & 3. 40076400 \\
\hline H & 1. 43740500 & -5.08700700 & -0.26680700 \\
\hline H & -0.16286700 & -5.96468200 & 4. 41140600 \\
\hline $\mathrm{H}$ & -0.08556500 & -3.13029500 & 4. 52564200 \\
\hline H & 2. 12381000 & -2.59720200 & -2.21279300 \\
\hline H & 0.50270300 & -1.19786800 & 3. 01167100 \\
\hline H & -1.14194600 & -0.94405400 & 0.69557500 \\
\hline $\mathrm{H}$ & 6. 04073200 & -1.63118600 & 0.30628100 \\
\hline H & 4. 17872400 & 0.94810600 & -2.57063100 \\
\hline H & 3. 62861700 & 1. 66806200 & 1. 60100300 \\
\hline $\mathrm{H}$ & -6.02817000 & -1.00474400 & -0.64858000 \\
\hline $\mathrm{H}$ & -3.95632000 & 1. 01062000 & 2. 51950700 \\
\hline H & -3.54743300 & 2. 38763400 & -1.49996300 \\
\hline $\mathrm{C}$ & 5. 86325500 & -1.28461000 & -2.38155000 \\
\hline $\mathrm{C}$ & 7. 39632600 & -1.09388600 & -2.27961900 \\
\hline H & 7. 90813400 & -1.67265300 & -3.05871500 \\
\hline H & 7. 78111900 & -1.42401400 & -1.30880400 \\
\hline $\mathrm{H}$ & 7. 66835000 & -0.03955800 & -2.40457400 \\
\hline $\mathrm{C}$ & 5. 53049700 & -2.78540100 & -2.21530500 \\
\hline H & 6. 03245000 & -3.37409300 & -2.99276100 \\
\hline $\mathrm{H}$ & 4. 45251300 & -2.96318500 & -2.30460400 \\
\hline $\mathrm{H}$ & 5. 85252700 & -3.17616600 & -1.24493000 \\
\hline $\mathrm{C}$ & 5. 41822200 & -0.85349500 & -3.79220800 \\
\hline $\mathrm{H}$ & 5. 69427000 & 0.18424400 & -4.00925000 \\
\hline $\mathrm{H}$ & 4. 33485900 & -0.95257700 & -3.92579600 \\
\hline H & 5.90500800 & -1.48622200 & -4.54305500 \\
\hline $\mathrm{C}$ & -5.15970600 & 0.58522000 & -2.76511800 \\
\hline $\mathrm{C}$ & -6.09695200 & -0.58958100 & -3.10412800 \\
\hline H & -5.64715100 & -1.55644900 & -2.85088800 \\
\hline $\mathrm{H}$ & -6.30324900 & -0.59629300 & -4.18047700 \\
\hline $\mathrm{H}$ & -7.05898300 & -0.51038700 & -2.58486800 \\
\hline $\mathrm{C}$ & -3.85508200 & 0.40845500 & -3.57806900 \\
\hline $\mathrm{H}$ & -3.34993200 & -0.52616900 & -3.31016600 \\
\hline H & -3.14988700 & 1. 22770300 & -3.40560900 \\
\hline
\end{tabular}


$-4.65196000$

$-5.68006200$

$-1.14272500$

2. 03916700

$-7.22365200$

$-1.06319500$

1. 97387200

$-7.67306700$

$-1.78971200$

2. 66224000

$-7.60155200$

$-1.27983900$

0. 96910800

$-7.57765000$

$-0.06450000$

2. 25484700

-5. 22225100

$-2.56981400$

1. 65515800

$-5.57137800$

$-2.85188000$

0. 65587800

$-5.62402800$

-3. 30457400

2. 36410500

-4. 12944300

$-2.64585700$

1. 66180300

-5. 25020500

$-0.88232400$

3. 49574100

$-5.56592300$

0. 10759700

3. 84466700

-4. 16405800

$-0.95781000$

3. 61853900

$-5.71105600$

$-1.62697000$

4. 15487500

5. 17217100

-0. 44827200

2. 58900300

4. 80156400

$-1.93041200$

2. 82909500

3. 73159300

$-2.10175300$

2. 66863300

5. 04030800

$-2.21680200$

3. 86093500

5. 35282100

$-2.60542900$

2. 16617500

4. 40015500

0. 40462000

3. 61416200

3. 31611500

0. 31588300

3. 48115100

4. 66640700

1. 46569100

3. 55079800

4. 64067800

0. 06657600

4. 62869100

6. 68714200

$-0.25354200$

2. 84071000

7. 29218400

$-0.87286300$

2. 16969500

6. 94275900

$-0.52884700$

3. 87153200

6. 98028200

0. 79120500

2. 68516500

$-5.85981500$

1. 89507800

$-3.20080100$

$-5.22500600$

2. 77098300

$-3.03209800$

-6. 79191600

2. 04539600

$-2.64374000$

-6. 10389800

1. 86057800

$-4.26990000$ 


\section{References}

1 (a) Lee, C.; Yang, W.; Parr, R. G. Development of the Colic-Salvetti CorrelationEnergy Formula into A Functional of the Electron Density. Phys. Rev. B. 1988, 37, 785789. (b) Becke, A. D. A New Mixing of Hartree-Fock and Local Density-Functional Theories. J. Chem. Phys. 1993, 98, 1372-1377. (c) Becke, A. D. Density-Functional Thermochemistry. III. The Role of Exact Exchange. J. Chem. Phys. 1993, 98, 56485652.

${ }^{2}$ Grimme, S.; Antony, J.; Ehrlich, S.; Krieg, H. A Consistent and Accurate ab initio Parametrization of Density Functional Dispersion Correction (DFT-D) for the 94 Elements H-Pu. J. Chem. Phys. 2010, 132, 154104.

3 Marenich, A. V.; Cramer, C. J.; Truhlar, D. G. Universal Solvation Model Based on Solute Electron Density and on a Continuum Model of the Solvent Defined by the Bulk Dielectric Constant and Atomic Surface Tensions. J. Phys. Chem. B. 2009, 113, 63786396.

${ }^{4}$ CYLview, 1.0b; Legault, C. Y. Université de Sherbrooke, 2009.

(http://www.cylview.org). 